\title{
SCALE MODEL SHAKE TABLE TESTING OF UNDERGROUND STRUCTURES IN SOFT CLAY
}

\author{
A Thesis \\ Presented to the Faculty of \\ California Polytechnic State University \\ San Luis Obispo \\ In Partial Satisfaction \\ of the Requirements for the Degree of \\ Master of Science in Civil and Environmental Engineering
}

By

Victor Anthony Crosariol

June 2010 
(C) 2010

Victor Anthony Crosariol

ALL RIGHTS RESERVED 


\section{COMMITTEE MEMBERSHIP}

TITLE:

AUTHOR:

DATE SUBMITTED:

COMMITTEE CHAIR:

COMMITTEE MEMBER:

COMMITTEE MEMBER
Scale Model Shake Table Testing of Underground Structures in Soft Clay

Victor Anthony Crosariol

June 2010
Charles Chadwell, Associate Professor

Gregg Fiegel, Professor 


\begin{abstract}
Scale Model Shake Table Testing of Underground Structures in Soft Clay

By Victor Anthony Crosariol
\end{abstract}

Underground structures perform an important role in transportation systems in many seismically active regions around the world, but empirical data regarding the seismic behavior of these structures is limited. This research works towards filling that empirical gap through the use of scale model shake table testing. Underground seismic soilstructure interaction (USSSI) effects were investigated for a stiff rectangular tunnel crosssection embedded within soft clay. San Francisco Young Bay Mud was used as a prototype soil for developing a scale model soil mixture consisting of kaolinite, bentonite, class C fly ash, and water. A single cell Bay Area Rapid Transit (BART) cut-and-cover subway tunnel was used as the prototype for the $10^{\text {th }}$ scale model subway cross-section. A flexible walled test container originally developed for a pile study at UC Berkeley was modified for use at Cal Poly, San Luis Obispo. The flexible container allows for close approximation of one-dimensional (1D) free-field site response by significantly limiting the rigidity of the boundary conditions and allowing the soil to deform under simple shear. The study was conducted over two shake table testing phases: Phase I consisted of shaking a model soil column to evaluate the ability of the test container to produce adequate 1D free-field site response, and Phase II tests explored the horizontal racking distortion of a shallow rectangular tunnel cross-section subjected to strong transverse ground shaking. Phase I test results and comparison with SHAKE models indicate that the test container can sufficiently mimic 1D free-field conditions, specifically for the 
primary shear deformation mode. Similarly, the equivalent linear soil-structure interaction code FLUSH was found to adequately model site response for the Phase II soil-structure system. Comparison of recorded horizontal racking distortions of the model structure with those from numerical modeling suggest that current simplified design methods may overestimate distortions to some degree for cases similar to those examined in this research. Overall, the flexible wall testing container shows promise as a viable means for gaining further insight into USSSI topics, as well as various other geotechnical and soil-structure interaction problems. 


\section{ACKNOWLEDGEMENTS}

I would like to extend my sincere gratitude to my adviser Prof. Robb Moss. His guidance, encouragement, and patience were invaluable through the many ups and downs during this research project. He gave me the latitude I needed to make this project my own, while providing the input necessary to move me in the right direction.

My other thesis committee members, Prof. Charles Chadwell and Prof. Gregg Fiegel, deserve a world of thanks for taking the time to read and review this long paper. Prof. Chadwell provided me with the help and knowledge I needed to get all the equipment and

instrumentation to function correctly, for which I am extremely grateful. Prof. Fiegel and the entire faculty here at Cal Poly have provided me with a valuable knowledge base to begin the next phase of my life.

Many friends and colleagues supplied assistance in all aspects of this project, and for that I am tremendously appreciative. Fellow researchers Steven Kuo and Gordon Bowman gave hundreds of hours of their time to the success of this project. They crawled in clay, crunched numbers, and were always willing help when I needed it the most. Structural gurus Matt Porter and Horacio Heredia provided me with much needed guidance for my structures related questions. Lucy Lo, Jackie Ng, Etheline Cimatu, Wilson Wong, Joe Heavin, and many others offered help and encouragement for which I am indebted.

I want to thank Xi Shen for dragging the mixer from place to place whenever it got in the way, and for his constant assistance with the general logistics of the project. 
This research project was funded by the Department of the Navy, Office of Naval Research, under Award \# N00014-07-1-1152 and Award \# N00014-08-1-1209. Without this generous financial support, this project would not have been possible. Also, I want to thank the University of California, Berkeley for loaning the heavy equipment needed to get a project of this size off the ground. Dr. Philip Meymand deserves my gratitude for writing an extremely in depth dissertation which provided a solid foundation for this project and hopefully many more future shake table investigations at Cal Poly.

I do not know how to express my gratitude to my friends and family for their support and encouragement, through times of doubt and times of joy. My good friends made me laugh and motivated me with every clever quip geared toward my status as a seventh year Cal Poly student. My older brothers Joe and Dom always pulled me back to earth for some good times during my short vacations away from this research. And of course my Mom and Dad gave me all the motivation I needed with their unconditional love, support, and assurance.

Thank you to everyone who kept me sane and on track through this long and fulfilling academic journey. 


\section{TABLE OF CONTENTS}

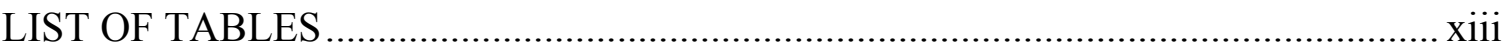

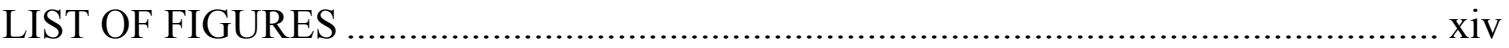

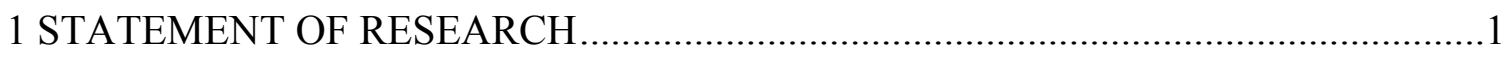

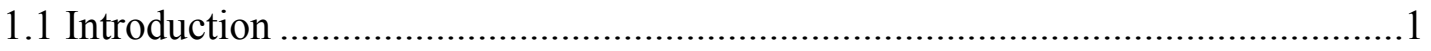

1.2 Seismic Soil-Structure Interaction of Underground Structures...............................1

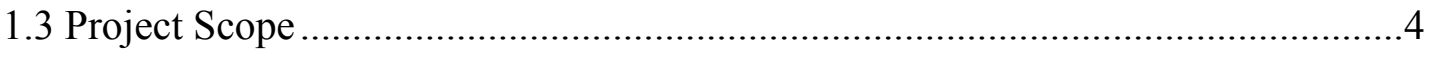

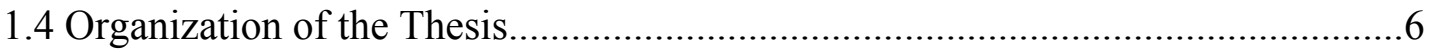

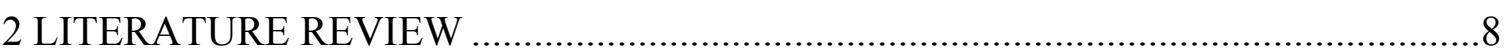

2.1 Analysis and Design of Cut-and-Cover tunnels ...............................................

2.1.1 Dynamic Earth Pressure Methods............................................................. 9

2.1.2 Free-Field Deformation Method ............................................................. 11

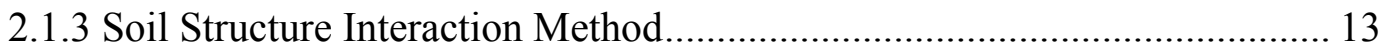

2.1.4 Simplified Frame Analysis .................................................................... 19

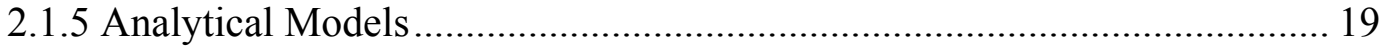

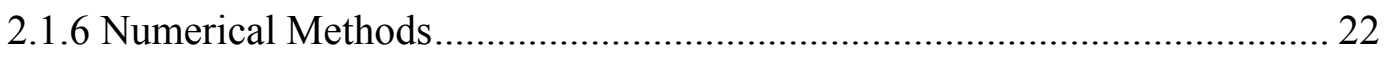

2.2 Case Studies of Damage to Tunnels .................................................................23

2.2.1 Collapse of Daikai Subway Station in Japan ............................................. 23

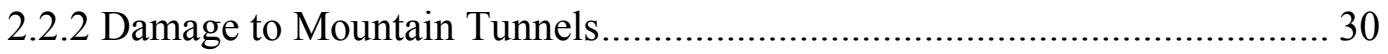

2.2.3 Damage to the Bolu Highway Twin Tunnels ............................................... 39

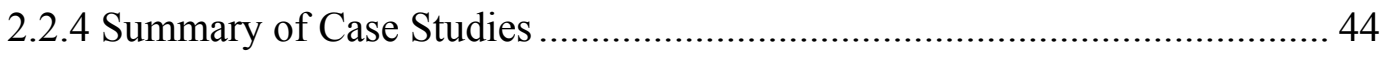

2.3 Previous Experimental work …………………...............................................45 
2.3.1 Experiments on Underground Structures .................................................... 46

2.3.2 1-g Scale Model Testing of Soft Clay........................................................... 51

2.3.3 Summary of Previous Experimental Work .................................................. 52

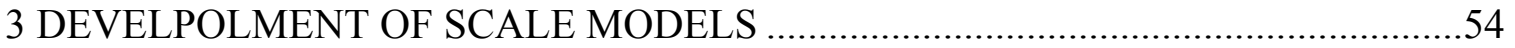

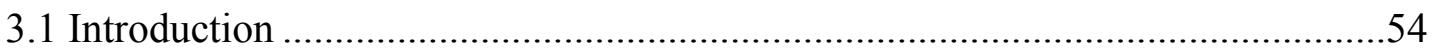

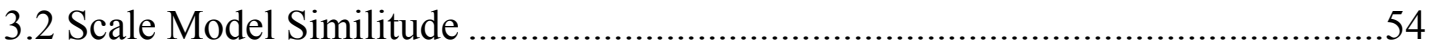

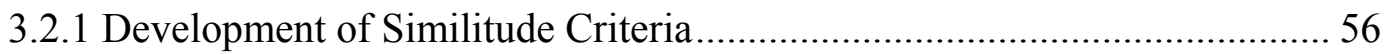

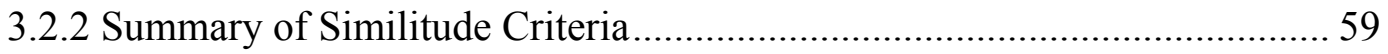

3.3 Development of Scale Model Soil..................................................................61

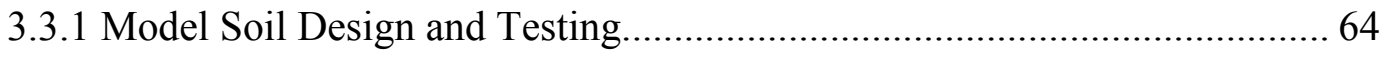

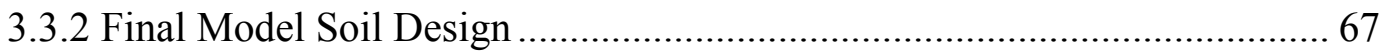

3.4 Development of Scale Model Tunnel...........................................................68

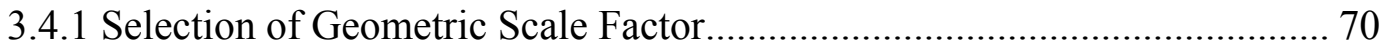

3.4.2 Scale Modeling Criteria for Tunnel Section .............................................. 71

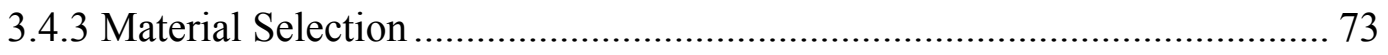

3.4.4 Final Structural Configuration ................................................................. 75

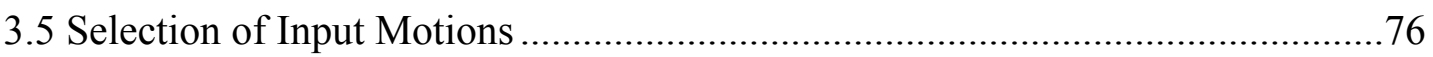

3.6 Scale Model Testing Container .......................................................................78

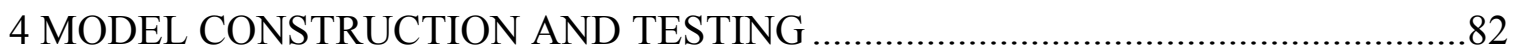

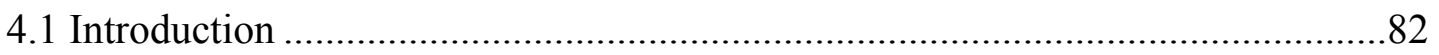

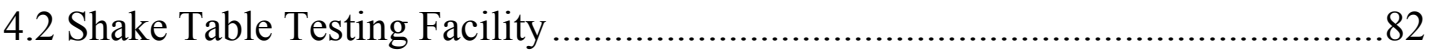

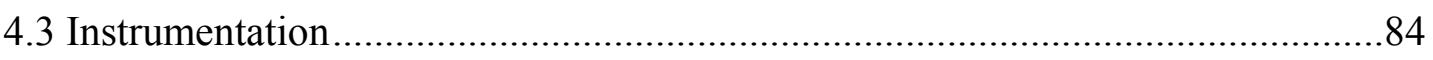

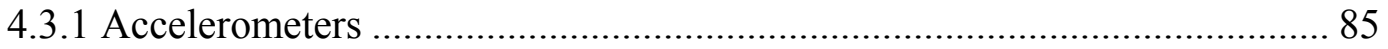




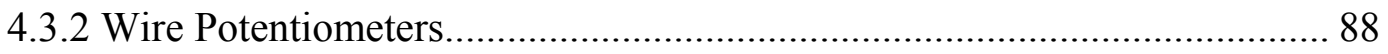

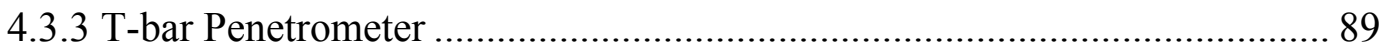

4.3.4 Linear variable differential transformers (LVDT's) .................................... 91

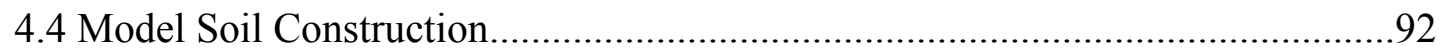

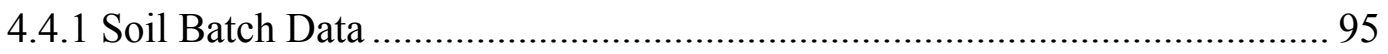

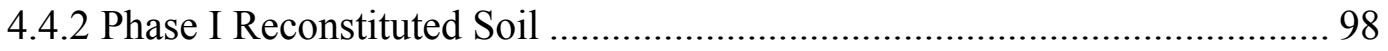

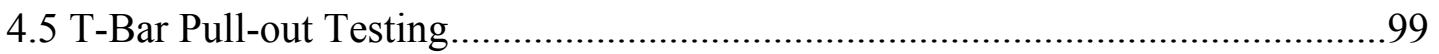

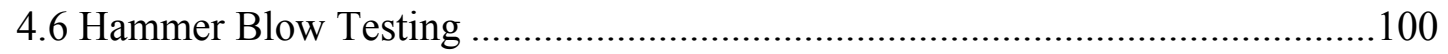

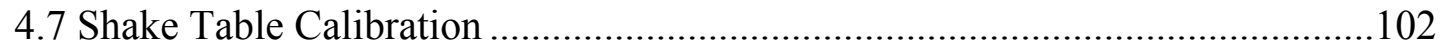

4.8 Structural Subway Model Construction and Placement......................................103

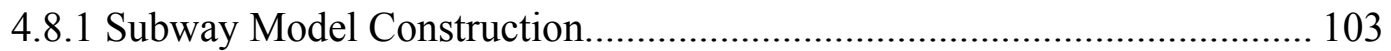

4.8.2 Subway Model Instrumentation................................................................ 105

4.8.3 Subway Embedment Procedures.............................................................. 107

4.9 Scale Model Testing Schedule ………………...............................................109

4.10 Shake Table Instrumentation Configuration ..................................................111

5 EXPERIMENTAL TESTING RESULTS …………………………………….....115

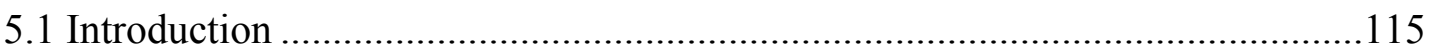

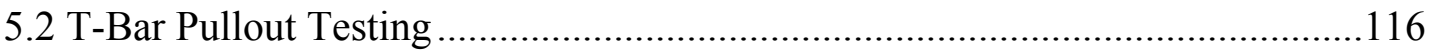

5.2.1 Comparison of Phase I and Phase II T-bar Results.................................. 118

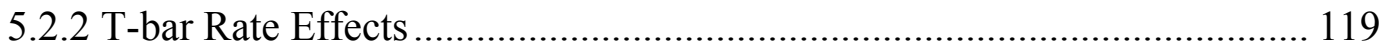

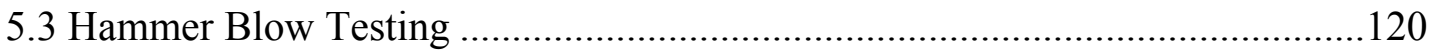

5.3.1 Bottom-up Hammer Blow Testing (series HB1) ...................................... 121

5.3.2 Top-down Hammer Blow Testing (series HB2) ...................................... 122 
5.3.3 Top-down Hammer Blow Testing (series HB3)....

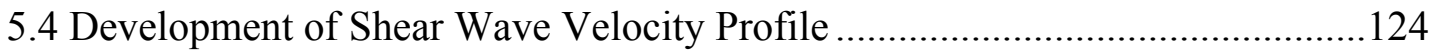

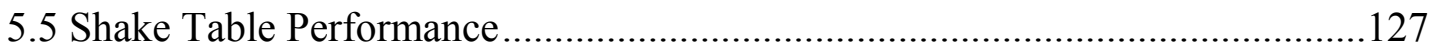

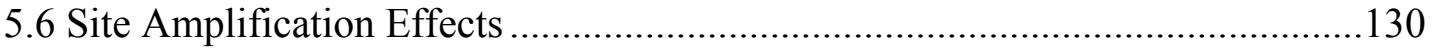

5.7 Testing Container Performance .................................................................. 132

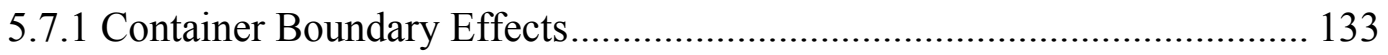

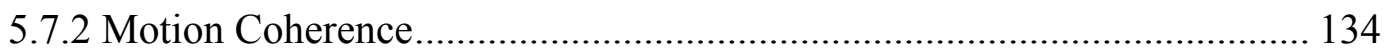

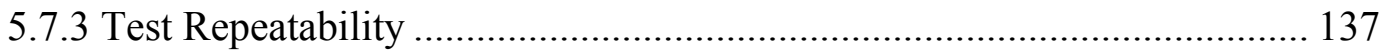

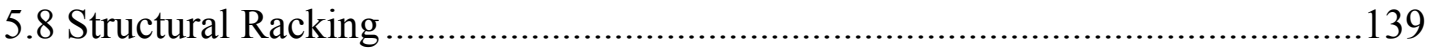

5.9 Phase I Free-Field Results Summary ...................................................... 144

5.10 Phase II USSSI Results Summary ............................................................. 145

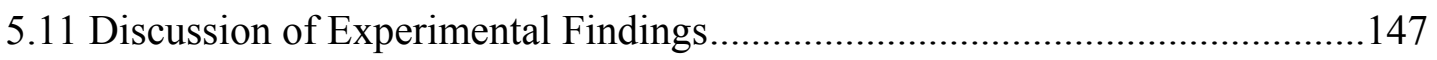

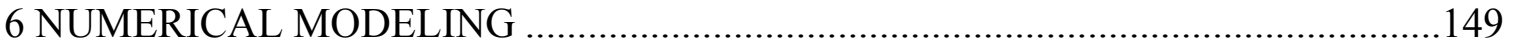

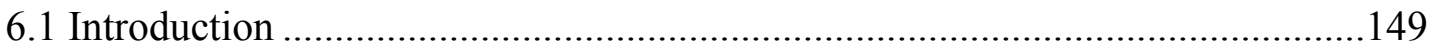

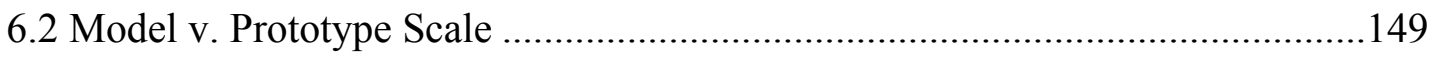

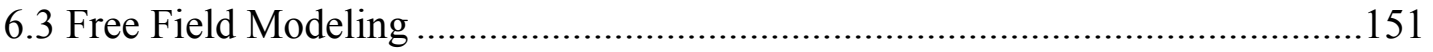

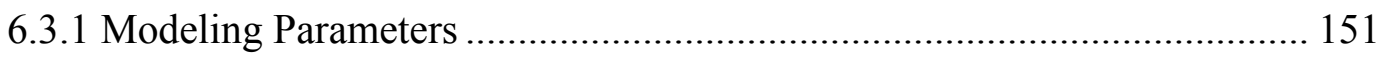

6.3.2 SHAKE Results and Discussion ...................................................... 154

6.3.3 Free-Field Racking Analysis....................................................... 157

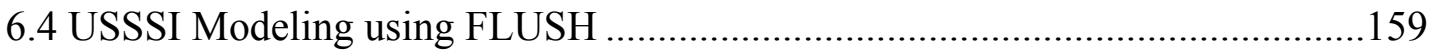

6.4.1 Modeling Parameters and Methodology ............................................. 159

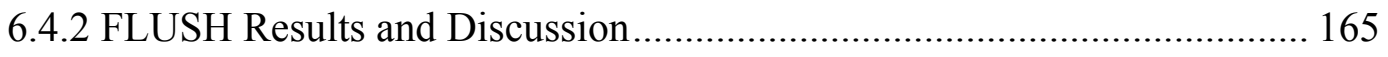

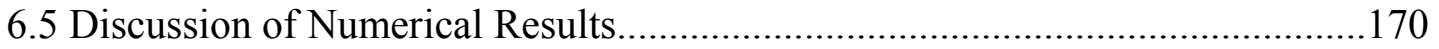


7 SUMMARY AND CONCLUSIONS

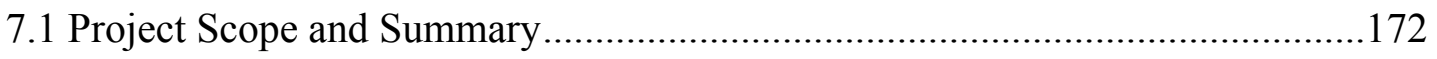

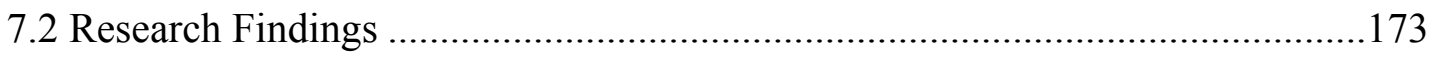

7.3 Improvement of the Testing Platform …………….........................................175

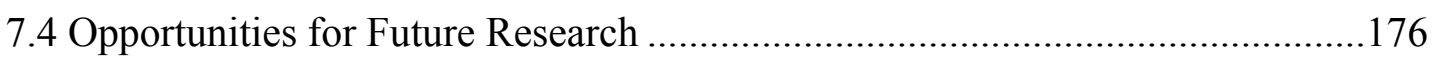

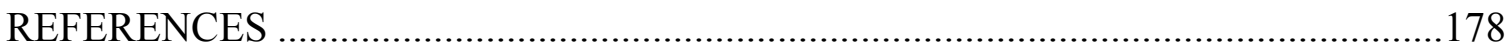

APPENDIX A - Detailed Material Mixing and Placement Procedures .......................... 184

APPENDIX B - Acceleration Data for Phase I Free-Field Testing............................... 192

APPENDIX C - SHAKE Modeling of Phase I Free-Field Motions .................................. 199

APPENDIX D - SHAKE91 Input Time History Error Report: October 6, 2009 ........... 203 


\section{LIST OF TABLES}

Table 3-1: USSSI components and associated variables (adapted from Meymand, 1998)

Table 3-2: Scale factors of selected engineering variables in terms of the geometric scaling factor $(\lambda)$ (adapted from Iai, 1989; Meymand, 1998).

Table 3-3 Selected properties for prototype and model soil mix in SSPSI study (after Meymand 1998) .66

Table 4-1 Selected accelerometer specifications .86

Table 4-2 Final Phase I testing schedule. 110

Table 4-3 Final Phase II testing schedule 110

Table 4-4 Phase I and Phase II instrumentation listing and nomenclature

Table 5-1 Summary of top down hammer blow testing for test series HB2 in the bottom half of the soil column

Table 5-2 Summary table of Phase I testing results at the bottom (1C) and top $(5 \mathrm{C})$ of the free-field soil column

Table 5-3 Summary table of Phase II testing results at the bottom (1C) and top (5C) of the soil column with the embedded model structure

Table 5-4 Summary table of Phase II structural racking results

Table 6-1 Results of simplified racking analysis using SHAKE and the racking relationship in Figure 6.3

Table 6-2 Material properties of the subway cross-section for use in FLUSH

Table 6-3 Racking distortion comparison of actual test data with FLUSH

simulations

Table 6-4 Summary of normalized racking distortions 


\section{LIST OF FIGURES}

Figure 1.1 - Schematic of USSSI response modes of a simple underground structure

Figure 2.1 - Structural Racking of a Buried Rectangular Frame Based off of the Free-Field Deformation Approach (from Hashash et al. 2001).

Figure 2.2 - General rectangular case for evaluating the flexibility ratio: distortions of a) free-field soil element and b) rectangular frame (from J.J. Wang, 1993)

Figure 2.3 - Normalized structural deflections shown for rectangular structural linings superimposed with closed form solutions for circular linings (from Hashash et al. 2001)

Figure 2.4 - Normalized racking deformations with respect to the flexibility ratio from multiple researchers (from Huo et al. 2006)

Figure 2.5 Damage to unconfined interior columns, leading to roof slab collapse in the Daikai subway station (from Sitar 1995)

Figure 2.6 - Ground subsidence on road above the Daikai station (from Sitar 1995)

Figure 2.7 - Damage patterns observed in the Daikai station collapse showing a) crack patterns in the interior ceiling slab (small numbers are crack width in mm) and b) elevation view of collapsed columns and ceiling slab (after Iida et al. 1996) ........26

Figure 2.8 - Soil-structure interface friction effects on soil modulus degradation for a) no-slip condition and b) full-slip condition where contours are $G / G_{\max }$ (after Huo et al. 2005).

Figure 2.9 - Photograph of the Chi-Shue tunnel portal (a) before and (b) after the 1999 Chi-Chi earthquake (from W.L. Wang et al. 2001).

Figure 2.10 Damage to the old Sanyi No. 8 south portal after the 1935 HsinchuTaichung earthquake (from Hwang and Lu 2007)....

Figure 2.11 - Summary of damage to mountain tunnels in Japan during major earthquakes (from Yashiro et al. 2007)

Figure 2.12 - Damage patterns observed in mountain tunnels (after Yashiro et al. 2007)

Figure 2.13 - Shearing of Longchi tunnel lining due to fault displacement

Figure 2.14 - Schematic of the tunnel cross-section though fault gouge clay, showing backfilled pilot holes and main tunnel linings (from Kontoe et al. 2008). 
Figure 2.15 - Accelerogram used in 2D finite element analysis (from Kontoe et al. 2008)

Figure 2.16 - Experimental results showing a) the relationship between sidewall curvature and dynamic earth pressures and b) observed sidewall cracking pattern (from Matsui et al. 2004)

Figure 2.17 - a) cyclic testing platform b) example double-cell cross-section investigated c) experimental hysteretic results compared with analytical results (after Nam et al. 2006)

Figure 2.18 - Numerical comparison of the free-field response of model containers with the response of a prototype soil column of soft clay (after Meymand 1998)

Figure 3.1 - Flowchart describing the method of implied prototypes, used to develop the model soil (from Meymand 1998).

Figure 3.2 - Relationship between shear wave velocity and static undrained shear strength of shallow cohesive soils (from Dickenson 1994).

Figure 3.3 - Typical double cell cut-and-cover subway cross-section (from BART)

Figure 3.4 - Typical single cell cut-and-cover BART cross-section with upper corner haunches (from Ostadan and Penzien 2001) .....

Figure 3.5 - Pseudo-static analysis results of influence of soil wedge reaction on the structural sidewall at the edge of the test container .70

Figure 3.6 - Model scale subway cross section design and associated engineering parameters .76

Figure 3.7 - 1979 Imperial Valley earthquake time histories, both horizontal azimuths of the El Centro motion corrected for full reflection with the time step scaled by $\lambda^{0.5}$

Figure 3.8 - 1992 Landers earthquake time histories, both horizontal azimuths of the Joshua Tree motion corrected for full reflection with the time step scaled by $\lambda^{0.5}$

Figure 3.9 - 1999 Chi Chi earthquake time histories, both horizontal azimuths of the TCU075 motion corrected for full reflection with the time step scaled by $\lambda^{0.5}$ .78

Figure 3.10 - Fully assembled testing container mounted to the shake table (the cross-braces are removed during testing)

Figure 4.1 - Interior overview of Parson's Earthquake and Geotechnical Engineering Laboratory 
Figure 4.2 - Signal conditioning and data acquisition module with instrumentation wiring

Figure 4.3 - Accelerometers used in this test program: (a) 393B04 ICP accelerometer mounted to and acrylic foundation and protected from moisture and chemical attack using 100\% silicone (b) J353B51 ICP accelerometers mounted within model structure (c) 353B52 ICP accelerometer mounted to shake table. .86

Figure 4.4 - Four wire potentiometers mounted off of shake table

Figure 4.5 - T-bar testing device with (a) $2.2 \mathrm{kN}$ load cell threaded to T-bar rod and eye bolt for pulling and (b) T-bar penetrometer cross-bar

Figure 4.6 - Linear Variable Differential Transformer (LVDT) used to measure racking displacement shown mounted within model structure

Figure 4.7 - ChemGrout mixer used to mix model soil .93

Figure 4.8 - Schematic of ChemGrout Mixer (after Meymand 1998).....

Figure 4.9 - Material immediately after discharge from mixer, ready to be hand packed .95

Figure 4.10 - Percent contribution of soil lifts to overall soil column volume .96

Figure 4.11 - Model soil water content at time of placement for a) Phase I and b)

Phase II.

Figure 4.12 - Unit weight measurements for as placed Phase I model soil units .98

Figure 4.13 - Deformed Phase I free-field model before soil reconstitution

Figure 4.14 - Top-down hammer blow testing using a framing hammer 101

Figure 4.15 - Fully assembled model structure with soil barriers and flexible tubing to accommodate instrumentation wiring

Figure 4.16 - Instrumented model structure without soil barriers

Figure 4.17 - Positioning of subway model in testing container using the overhead crane (shaking direction in photograph is approximately left to right) 108

Figure 4.18 - Phase I soil column instrumentation configuration.................................112

Figure 4.19 - Phase II soil column instrumentation configuration ................................113

Figure 4.20 - Phase II subway cross-section instrumentation configuration ...................114

Figure 5.1 - Phase 1 T-bar testing results ............................................................. 117

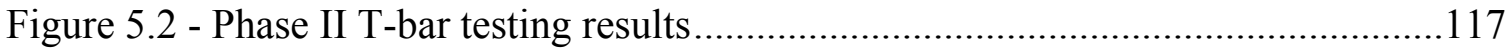


Figure 5.3 - Average undrained shear strength of the soil column using T-bar tests ......118

Figure 5.4 - Effect of penetrometer shearing rate on undrained shear strength measurements (from Meymand 1998)....

Figure 5.5 - Typical bottom-up hammer blow testing waveforms generated in the center accelerometer array (test series HB1)

Figure 5.6 - Typical wave forms from test series HB2 for the full soil column

Figure 5.7 - Typical wave forms from test series HB2 for bottom half of soil profile (arrows indicate the first wave arrival used for calculating shear wave velocity)

Figure 5.8 - Composite shear wave velocity profile for the Phase I soil column

Figure 5.9 - Best estimate shear wave velocity profile for both testing phases composed from $\mathrm{T}$-bar and hammer blow data

Figure 5.10 - 5\% damped response spectra comparing desired input motion and actual recorded output motion for both Phase I free-field Joshua Tree motions

Figure 5.11 - Comparison of input and output table displacement time histories for TCU075 North (Test 2_CN.3)

Figure 5.12 - Comparison of input and output table displacement time histories for El Centro 180 (Test 2_E180.3)

Figure 5.13 - Acceleration and 5\% damped response spectra recorded at the center accelerometer array for the free-field Joshua Tree 000 motion (test 1_J000)

Figure 5.14 - Ratio of maximum 5\% damped spectral acceleration of the wall array relative to the center array

Figure 5.15 - 5\% damped response spectra for Phase I El Centro testing at an elevation of $55 \mathrm{~cm}$ in the soil column

Figure 5.16 - 5\% damped response spectra for the Phase II TCU075N motion at elevations of $10 \mathrm{~cm}, 55 \mathrm{~cm}$, and $100 \mathrm{~cm}$ in the soil column.

Figure 5.17 - Test repeatability illustrated by $5 \%$ damped response spectra at the table and soil column surface (position 5C) for both Phase II TCU075 motions.

Figure 5.18 - First 3 seconds of the Joshua Tree 000 racking time history illustrating equal horizontal distortion recorded at each structural sidewall.

Figure 5.19 - Racking distortions for the TCU075 motion (test 2_CW.2) ....................140

Figure 5.20 - Racking distortions for the El Centro 270 motion (test 2_E270.3) ..........140 
Figure 5.21 - Ceiling slab acceleration time history overlaid with the LVDT East distortion recording for the TCU075 North motion (test 2_CN.2)

Figure 5.22 - Plot showing the relationship between maximum differential racking acceleration and maximum racking distortion

Figure 5.23 - 5\% damped response spectra within model structure (test 2_CW.2) .......143

Figure 6.1 - Modulus Reduction and Damping curves used in SHAKE analyses...........153

Figure 6.2-5\% damped response spectra for (a) TCU075 North (1_CN) and (b)

El Centro 180 (1_E180) center array recordings versus SHAKE predictions.

Figure 6.3 - A portion of the normalized racking relationship developed by J. J.

Wang (1993) with a curve fit for use in a spreadsheet solution.

Figure 6.4 - Finite element mesh used for USSSI simulations of the Phase II testing.....

Figure 6.5 - 5\% Damped response spectrum from test 2_J000.3 compared with free-field and USSSI test results

Figure 6.6 Comparison of testing and FLUSH racking distortions, normalized by the average values over all motions..... 168

Figure 6.7 - Normalized racking distortions obtained from numerical analysis (FLUSH) and test results (adjusted) compared with those from J. J. Wang (1993).

Figure A.1 - Valve set to CYCLE (one of two valves)............................................. 186

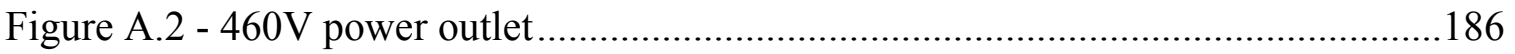

Figure A.3 - (a) Hydraulic controls and (b) Electrical controls .................................187

Figure A.4 - Mixing dry material in a wheel barrow and adding it to the tank .............187

Figure A.5 - Platform hung from crane for transporting material ...............................189

Figure A.6 - Discharge hose held and controlled using the overhead crane....................190

Figure A.7 - Valve set to DISCHARGE (one of two valves) ......................................190

Figure B.1 - Joshua Tree 000 acceleration time histories and 5\% damped response Spectra (test 2_J000) recorded in the center accelerometer array

Figure B.2 - Joshua Tree 090 acceleration time histories and 5\% damped response Spectra (test 2_J090) recorded in the center accelerometer array

Figure B.3 - El Centro 270 acceleration time histories and 5\% damped response Spectra (test 2_J090) recorded in the center accelerometer array .... 
Figure B.4 - El Centro 180 acceleration time histories and 5\% damped response Spectra (test 2_J090) recorded in the center accelerometer array

Figure B.5 - TCU075 North acceleration time histories and 5\% damped response Spectra (test 2_J090) recorded in the center accelerometer array

Figure B.6 - TCU075 West acceleration time histories and 5\% damped response Spectra (test 2_J090) recorded in the center accelerometer array 198

Figure C.1 - 5\% damped response spectra for a) Joshua Tree 000 (1_J000) and b) Joshua Tree 090 (1_J090) center array recordings versus SHAKE predicted response spectra

Figure C.2 - 5\% damped response spectra for a) El Centro 270 (1_E270) and b) El Centro 180 (1_E180) center array recordings versus SHAKE predicted response spectra .

Figure C.3 - 5\% damped response spectra for a) TCU075 North (1_CN) and b) TCU075 West (1_CW) center array recordings versus SHAKE predicted response spectra

Figure D.1 - Variation of 5\% SHAKE91 5\% damped response spectra with respect to number of columns in input time history .205 


\section{CHAPTER 1 - STATEMENT OF RESEARCH}

\subsection{Introduction}

Underground structures perform a vital role in railway and highway systems in many seismically active regions around the world. Despite their historic importance as critical infrastructure, they have only in recent decades gained special attention in the realm of seismic structural design. Furthermore, as tunneling and construction technology improves, design of tunnel facilities is becoming less constrained by geography and geology.

Tunnels can be built in just about any ground condition from shallow soft clays to deep intact bedrock, and in many different shapes and sizes. As a result, they must be able to accommodate a wide range of loading conditions such as static earth pressures, construction blasts, and seismic loading. Due to the possible risk to life safety, earthquakes are the most important source of dynamic loading to consider for underground structures. A common notion regarding underground structures is that they are safer than surface structures during earthquakes. This belief has been called into question as a result of recent failures of underground structures, notably the catastrophic collapse of the Daikai subway station in Japan during the 1995 Kobe earthquake. In order to mitigate the risk of earthquake damage, thorough understanding of the seismic response of underground facilities is needed.

\subsection{Seismic Soil-Structure Interaction of Underground Structures}

To accurately evaluate the seismic response of an underground structure, it is first important to understand that the behavior of the structure and the surrounding ground are 
not independent. The presence of a structure influences the seismic response of the ground; and likewise, the ground influences the seismic response of the structure. For soil, this coupled dynamic response between the structure and the ground is termed seismic soil-structure interaction (SSI). There are many examples of infrastructure that warrant seismic SSI research such as elevated highways, bridges, overpasses, water canals, water supply tunnels, pipelines, levee systems, dams, and underground transportation facilities. This research focuses on the latter, and will hereafter be referred to as Underground Seismic Soil-Structure Interaction (USSSI).

Seismic design of tunnels has historically been based on the understanding that the seismic performance of such facilities is mostly controlled by deformation and strain sustained by the structure. However, due to the complexity of characterizing USSSI effects, they have often been ignored in favor of imposing free-field ground deformations to estimate structural stresses and strains. Research by J.J. Wang (1993) showed that structural deformations do not usually mimic those of the free-field ground, especially in situations where a large stiffness contrast exists between a structure and the surrounding soil. Past studies indicate that the following factors influence USSSI effects:

- Kinematic interaction is characterized by the presence of a structure altering the response of the surrounding medium from that of the free-field response (Kramer 1996). It is essentially a function of the soil-structure stiffness contrast. The presence of a structure can amplify ground deformations beyond those of the freefield or conversely, may result in de-amplification of near-field ground motions. Deeply embedded structures behave unlike surface structures, in which seismic response is also affected by inertial forces imposed onto the foundation system by 
the mass of the superstructure. For most USSSI problems, inertial interaction is a minor concern.

- Damping is an important component of USSSI and can be described as the dissipation of seismic energy. The various forms of damping include, but are not limited to: material damping of the structure, hysteretic damping of the soil, and radiation damping. Radiation damping is the dissipation of energy into an unbounded volume, which may be considered the far-field condition for soil (Wolf and C. Song 2002). Meymand (1998) explains that radiation damping is most pronounced when soil damping is low and the frequency of vibration is high, given that no gapping occurs at the soil-structure interface.

- Shear and slippage along the soil-structure interface is an important consideration because the surface area of an underground structure can be substantial. This is a complex issue which plays a significant role in the deformation characteristics of a structure, and may also affect the behavior of near field soil.

- Critical interaction effects between a structure and its founding medium can also occur prior to seismic loading. For example, tunneling can result in disturbance of the in-situ medium, which may impose unanticipated stress states that are difficult to quantify. Stresses due to seismic loading will then be superimposed on these existing stress states (Meymand 1998). Adverse conditions such as gapping in cohesive soil can reduce confinement from the soil and decrease lateral stiffness of soil-structure system. 
When considered in combination, all of the factors that contribute to USSSI constitute a highly complex non-linear dynamic system. Such systems cannot readily be analyzed without the use of sophisticated, time-consuming finite-element analysis methods. Moreover, there may be uncertainty in the inputs required for complex SSI models that can lead to erroneous conclusions. Figure 1.1 shows a schematic of a simple USSSI problem and some coupled interaction modes.

\section{Ground Surface}

(Surface Boundary Effects for Very Shallow Structures)

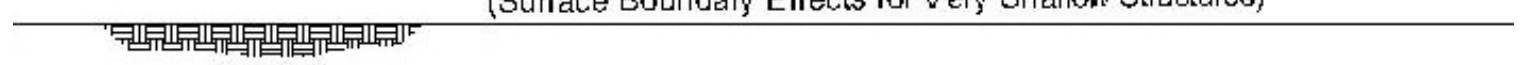

\section{Overburden Soil}

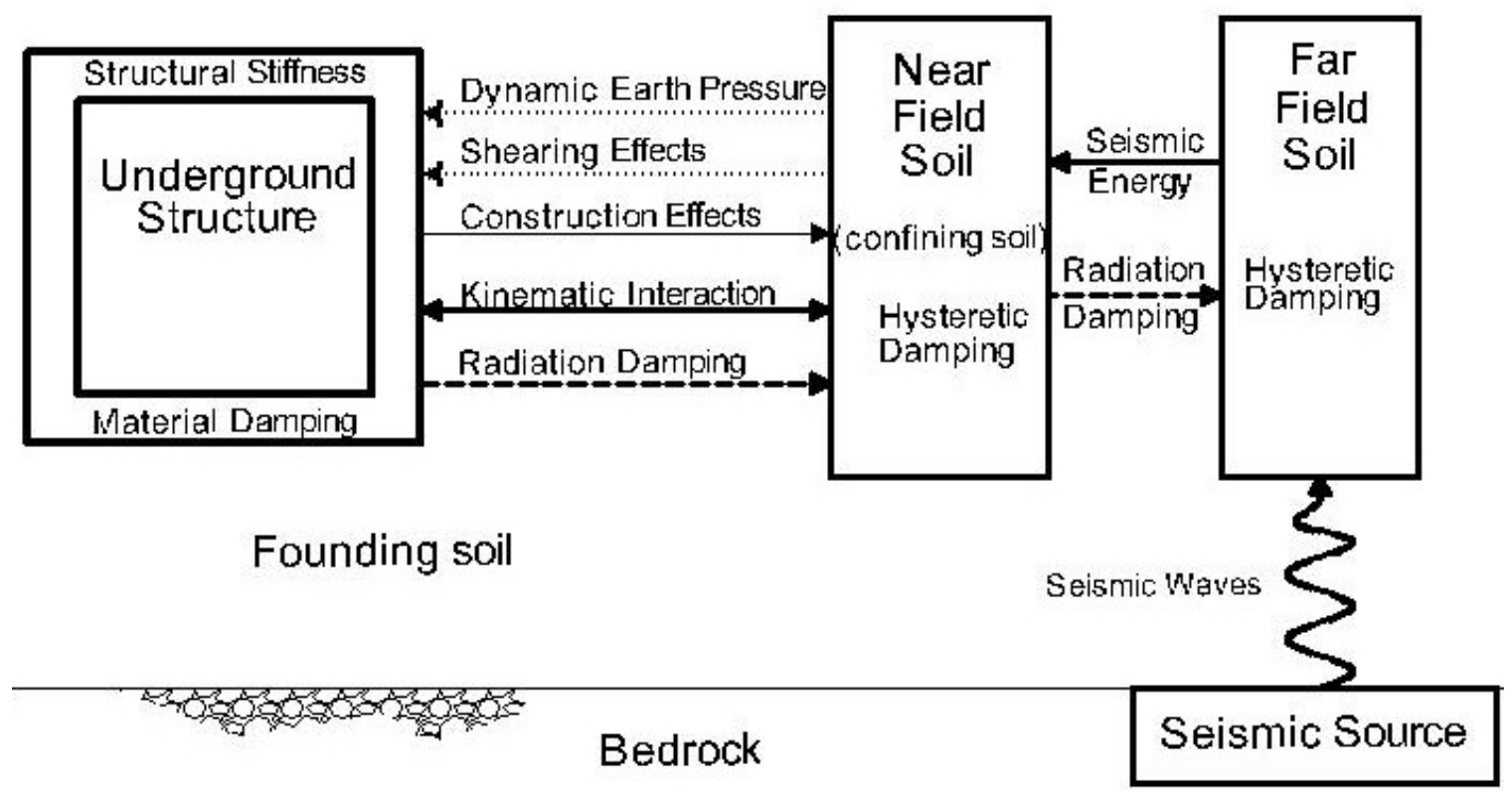

Figure 1.1 - Schematic of USSSI response modes of a simple underground structure

\subsection{Project Scope}

As will be discussed in Chapter 2, a limited number of well-documented cases exist regarding the behavior of underground facilities under seismic loading, and little 
physical testing has been performed to fill this knowledge gap. Much of the research in the area of USSSI effects have focused on finite element modeling of observed tunnel failures. Physical testing has mainly focused on the dynamic response of reinforced concrete structures in sand. Underground infrastructure built in soft clay is not uncommon in seismically active regions. Therefore, a need exists to increase empirical knowledge of the seismic response of tunnels. Empirical data may then be used to calibrate simplified analytical models and complex dynamic models. Examples of tunnels built in soft clay include: the San Francisco Bay Area where portions of the Bay Area Rapid Transit (BART) line are constructed in soft saturated clays, the Singapore Mass Transit Line (SMRT) where tunnels have been bored through soft marine clay (Hulme et al. 1990), and the Shanghai Yangtze River Tunnel which is excavated in soft and muddy clay beneath the Yangtze river in China (Li et al. 2009).

The purpose of this research project is to use physical shake table testing to investigate the coupled seismic soil-structure interaction between soft clay and a stiff rectangular tunnel section subjected to strong ground shaking transverse to the tunnel travel direction. A secondary goal is to develop a suitable physical testing platform and procedures for further SSI research beyond the realm of underground facilities. Shake table testing provides an opportunity to observe and quantify the response of coupled SSI systems in an environment where selected variables can be varied and controlled.

As a part of this research, shake table tests are performed on a scale model rectangular subway section subject to strong ground-shaking in the transverse direction. Acceleration and displacement data are collected from the structure and the soil for quantifying USSSI effects. The differential horizontal displacement between the top and 
bottom of the structural section is termed horizontal racking and is a key factor in quantifying the seismic response of tunnels. Racking deformations recorded during 1D shake table tests are then compared with numerically modeled deformations using the equivalent-linear SSI code FLUSH (Lysmer et al. 1975) for validation of the testing platform.

The following research objectives have been identified for the shake table testing platform and subsequent numerical analyses:

- develop a testing platform capable of closely mimicking free-field conditions of soft clay

- employ similitude laws to develop a scale model soil-structure system

- quantify coupled USSSI response of a rectangular tunnel section in soft clay

- compare the effect of different ground motions on a soil-structure system

- use numerical methods to validate the testing platform

- compare experimental racking results with results from numerical analyses

- develop a suitable framework for future SSI shake table testing at California Polytechnic State University, San Luis Obispo

\subsection{Organization of the Thesis}

Chapter 2 is divided into three main sections as follows: the first outlines the current state of practice for seismic design of underground rectangular tunnel facilities; the second surveys and compares a number of cases where damage to underground facilities has been observed as a result of major earthquakes; and, the third reviews a few selected studies where physical testing has been used to characterize seismic underground 
structural response. Chapter 3 provides an overview of the testing platform and its development, including scaling relations, the model soil and structure, and the testing container. Chapter 4 outlines procedures regarding placement of testing materials, test setup, and instrumentation placement for the purpose of providing useful information for future researchers looking to expand or improve on this testing platform. Also provided in Chapter 4 is detailed information regarding instrumentation and data acquisition. Chapters 5 and 6 provide quantitative results and discussion of results for the physical shake table testing and numerical analyses respectively. Finally, a discussion of research findings and recommendations for future research are provided in Chapter 7. 


\section{CHAPTER 2 - LITERATURE REVIEW}

\subsection{Analysis and Design of Cut-and-Cover tunnels}

Design of underground tunnel facilities is an important concern, especially due to the increasing importance of public transportation systems. The following sections review past, current, and state-of-the-art analysis and design methods regarding underground structures. Considered together, Hashash et al. (2001) and J.J. Wang (1993) provide a comprehensive summary of well-established design methods developed within the last two decades regarding many important of aspects of underground structural design. Additionally, a newly released report by the United States Department of Transportation Federal Highway Administration constitutes a practical design guide for road tunnels using the current state of practice (FHWA, 2009).

According to J.J. Wang (1993), sections of transportation systems built using the cut-and-cover method of construction are often in rectangular form. This is opposed to the circular tunnels most commonly found at deeper burial depth, which are often constructed using tunnel boring machines and blasting. Both circular and rectangular tunnel linings are subjected to transverse racking deformations resulting from shear distortions of the surrounding ground; however, their design methodologies can differ substantially due to various factors such as shape, ground conditions, and construction methods. The discussion herein focuses primarily on rectangular structures, but many of the concepts discussed translate in some degree to other kinds of structural sections. There are three important considerations that should be taken into account for rectangular cut-and-cover tunnels. First, the relatively shallow depth of these tunnels often subjects them to higher ground deformations and shaking intensities than those typically observed 
for deeper structures. This is due to site amplification effects and the relatively lower stiffness of shallow soils. Second, the box frame shape of cut-and-cover sections is not as efficient at transferring static loads as circular lined tunnels. This leads to relatively stiff section designs, which may have low tolerance to transverse ground deformation. Last, cut-and-cover sections are generally backfilled with compacted material on top and at the sidewalls of the structure. Any backfill properties should be properly accounted for in the design and analysis cut-and-cover structures. The study herein covers two of these three considerations. Specifically, it focuses on transverse deformation of a stiff rectangular, structure in shallow, soft clay.

Variable backfill properties can alter stress conditions by providing different stiffness contrasts and interface shear than free-field material. Also, compaction can lead to increased earth pressures on structural sidewalls. These are issues that would greatly complicate the modeling effort in this study. For this reason, the study herein does not examine the effect of variable backfill.

\subsubsection{Dynamic Earth Pressure Methods}

Some early methods for seismic design of underground facilities considered the dynamic pressure distributions imposed onto a structure under dynamic loading conditions. Seed and Whitman (1970) present the Mononobe-Okabe method for estimating dynamic lateral earth pressures on earth retaining structures adapted from earlier work by Mononobe and Okabe. Lateral pressures on earth retaining structures can increase dramatically under seismic and blast loading, making it an important design consideration. The Mononobe-Okabe method imposes a dynamic earth pressure 
distribution model onto the structural walls, resulting from inertial forces of the surrounding medium. Seed and Whitman warn in their study that increased dynamic lateral earth pressures on buried subway walls are "a special problem outside the scope" of their discussion. Despite this, the method has been used in design of underground subway structures and has previously been endorsed by the Japanese Society of Civil Engineers (JSCE, 1975).

J.J. Wang (1993) explains that the applicability of the Mononobe-Okabe method to underground rectangular shaped tunnels has been a subject of debate due to the assumption that an active soil wedge must yield and form behind the subway wall. The ground and structure generally move in phase for a buried underground section, reducing the possibility of an active soil wedge forming. The likelihood of an active wedge forming under dynamic conditions decreases with increasing burial depth. However, according to the Mononobe-Okabe method, the full soil surcharge pressures must be considered in the earth pressure distribution model. This can lead to unrealistically high distortion estimates as the burial depth of a structure increases. Applicability of this method is reasonable in situations where no surcharge is present or under minimal surcharge, as in very shallow tunnel sections. Transition sections without ceiling slabs are an example where the sidewalls behave similarly to typical earth retaining structures. Other dynamic earth pressure models exist, but contain similar drawbacks. Accurately evaluating the dynamic loading distributions on a buried tunnel requires complex dynamic analyses that can be more easily accomplished by approaching the horizontal racking problem from a deformation perspective, as opposed to a loading perspective. 


\subsubsection{Free-Field Deformation Method}

The free-field deformation approach, outlined by a number of researchers, is a common method that does not consider USSSI effects (Hashash et al. 2001; Kuesel 1969; Monsees and Merritt 1991; J. J. Wang 1993). Free-field deformations are seismically induced strains developed in a medium in the absence of structures and excavations. The method described here can be used as a first order analysis tool for estimating structural deformations. Structural distortion may be overestimated or underestimated depending on the relative stiffness of a structure and the surrounding medium. Important public transportation projects in California have historically been seismically designed based on free-field deformations (Hashash et al. 2001).

As reported by J.J. Wang (1993), Kuesel (1969) proposed a project specific design methodology for the San Francisco Bay Area Rapid Transportation (BART) project. This methodology for seismic design of tunnels involved evaluating the freefield deformations of an earth medium and imposing those deformations on the structure. In isolated cases where rotational plastic hinges might develop, special structural details were provided to mitigate damage to critical joints.

Similar methods were also employed in the Los Angeles Metro (L.A. METRO) project as described by Monsees and Merritt (1991). The design philosophy follows the suggestion that it is "always proper or conservative to assume that the structure deforms with the soil." This depends on the assumption that a structure will follow the deformation characteristics of the free-field medium. The design is conservative in the case where the structure is stiffer than the free-field soil, but more recent research shows that structural deformations can exceed free-field deformations in some situations (J. J. 
Wang 1993). Design for the L.A. METRO further builds on Kuesel's method in that it allows for the development of plastic hinges during a Maximum Design Earthquake (MDE). This calls for ductile reinforcement design at critical joints, effectively increasing the flexibility of the structure. Acceptable plastic hinge conditions are those which do not result in tunnel collapse. Unacceptable plastic hinge conditions exist when three plastic hinges develop in any single structural member.

Numerical analysis tools provide a convenient method for evaluating free-field shear distortions and may be necessary in complicated situations, such as those with variable soil layering (Hashash et al. 2001). Equivalent linear computer programs based on vertical wave propagation theory can be used, such as the one-dimensional (1D) code SHAKE (Schnabel et al. 1972) and the two-dimensional (2D) code QUAD4M (Hudson et al. 1994). The shear distortions can be plotted with depth once free-field strains are evaluated for a particular soil site. Relative shear deformation of the structure can then be estimated as the difference between the free-field distortion at the top and bottom of the structure, as illustrated by Figure 2.1.

Hashash et al. (2001) observes that the free-field design approach can be a useful tool in simple situations when ground deformations induced under seismic load are small. The method is most applicable when the surrounding medium is very stiff, shaking intensity is low, or the structure is relatively flexible. Under the aforementioned conditions, a well designed structure should be able to sufficiently absorb the resulting ground deformations without being overly stressed (J. J. Wang 1993). Issues with the method arise in situations where the medium is soft compared to the structure, as is common in shallow cut-and-cover rectangular sections. When applied in soft soil 
conditions, the method can likely result in unnecessary conservatism. This is most apparent in cases where the box structure is designed to be very stiff in order to withstand large static loads. Deformation in these stiff structures can be significantly less than that of the soft free-field soil medium (J. J. Wang 1993). This necessitates methods that consider USSSI effects with the intention of avoiding overly conservative designs.

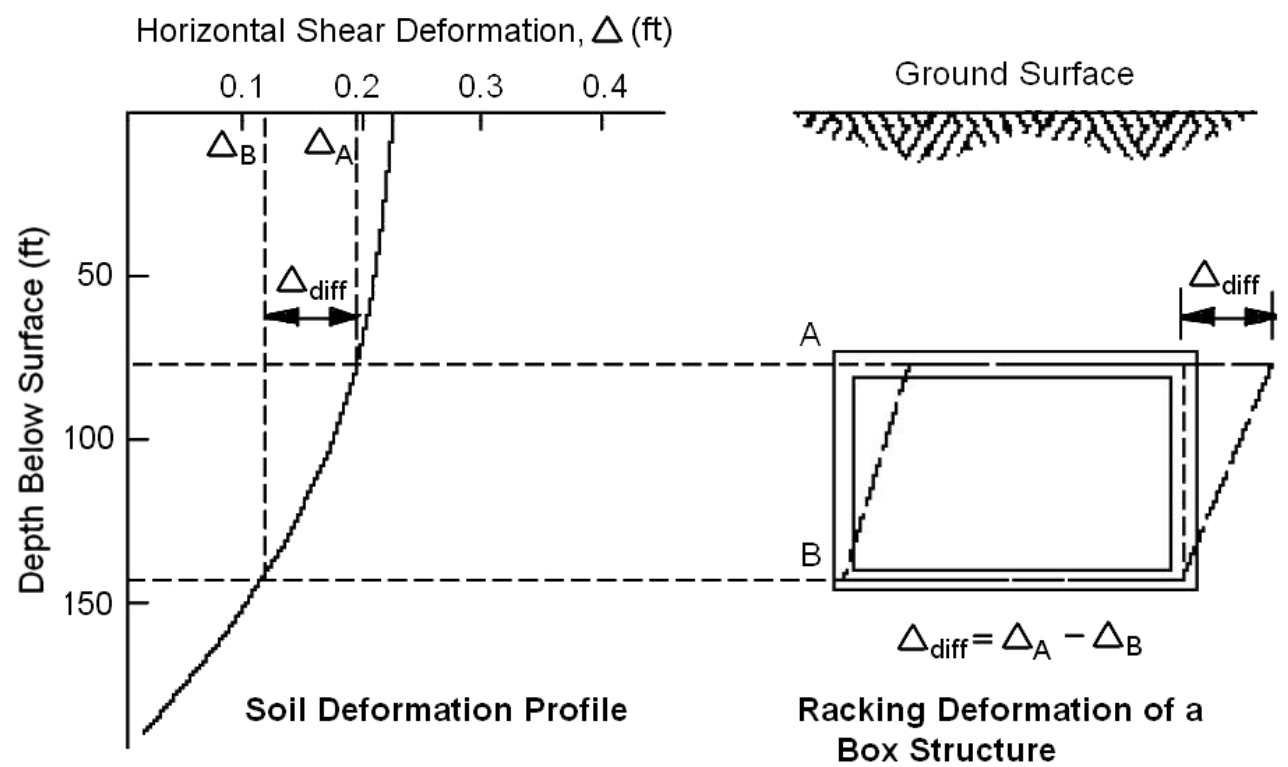

Figure 2.1 - Structural Racking of a Buried Rectangular Frame Based off of the FreeField Deformation Approach (from Hashash et al. 2001)

\subsubsection{Soil Structure Interaction Method}

A design method that considers USSSI effects has been developed and termed by J.J. Wang (1993) as Tunnel-Ground Interaction Analysis. It is summarized in the following section, and the results of the study provide a basis for current recommended design procedures regarding lateral racking in rectangular tunnels. The variable nature of the geometry of cut-and-cover section designs does not permit closed-form solutions like those available for circular lined tunnels. Therefore, it was desirable to develop a simple 
design method for taking into account USSSI effects regarding these types of facilities. Closed-form solutions for evaluating ovaling deformations of circular tunnels have been developed and presented by various researchers but are not discussed here (Hashash et al. 2001, 2005; Penzien 2000; Penzien and Wu 1998; J. J. Wang 1993).

To aid in the formulation of his design method, Wang performed dynamic finite element analyses on various structural sections. Results from the study were used to develop normalized chart-based solutions to be used in design practice for evaluating horizontal racking deformations in rectangular structures. The study was performed using the computer program FLUSH (Lysmer et al. 1975). FLUSH is a frequency domain, two-dimensional, equivalent linear, plane strain computer code. The code has two ideal features for running deformation analyses on underground facilities: (1) maximum relative distortion can be evaluated between any two locations within the soil-structure model and (2) it allows for free-field response analysis simultaneously for comparing the relative distortion between free-field conditions and the soil-structure model. Internal forces in structural members within the model can also be estimated using FLUSH (J. J. Wang 1993). The paper provides a useful blueprint on how to run dynamic analyses using FLUSH by providing important assumptions and detailed descriptions of experimental methods.

An advantage of rigorous dynamic analysis is that it can be used to generate data sets for calibration and validation of simplified design procedures. J.J. Wang (1993) uses dynamic numerical analyses to develop and calibrate such a procedure to account for USSSI effects. An extensive data set was developed through a total of 36 dynamic finite element model simulations. The primary focus of the study involved 25 simulations 
where relatively homogeneous soft soil overlies stiffer soil. The following factors identified as contributing to USSSI were varied parametrically in the simulation: relative stiffness between the soil and the structure, structural geometry, input earthquake motions, and tunnel embedment depth (J. J. Wang 1993).

The study considered five different structural sections varying in size and complexity: three single cell and two double cell sections. Two synthetically generated accelerograms were used as input motions to simulate typical western U.S. and northeast U.S earthquakes. The accelerograms were generated from outcrop response spectra and subsequently modified for suitability as base input motions using the de-convolution capabilities in the computer program SHAKE. Depth of embedment was varied between 15 and 22.5 feet for all cases (J. J. Wang 1993). This is relatively shallow and is typical of cut-and-cover rectangular subway sections and stations. Relative stiffness between the soil and structure is accounted for using a dimensionless quantity termed the flexibility ratio.

The flexibility ratio $(F)$ is the relative stiffness of the free-field medium in simple shear to that of the structure replacing it. Considering the general rectangular soil element subjected to simple shear deformation shown in Figure 2.2a, the flexibility ratio is defined as follows (Hashash et al. 2001):

$$
F=\frac{G_{m} L}{S_{1} H}
$$

where $G_{m}$ is the stiffness in simple shear of the rectangular soil element, $L$ and $H$ are the width and height respectively of the rectangular structure (and soil element), and $S_{1}$ is the unit racking stiffness, or the concentrated force needed to result in a unit deflection of the 
structure $\left(S_{1}=1 / \Delta\right)$. The full derivation of the flexibility ratio is available in J.J. Wang (1993) and Hashash et al. (2001).

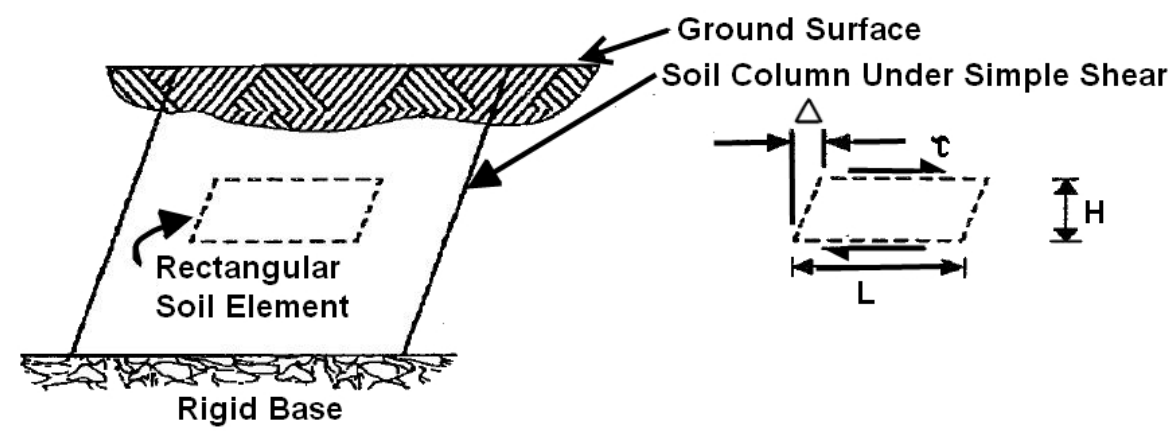

A. Flexural (Shear) Distortion of a Free-Field Soil Medium

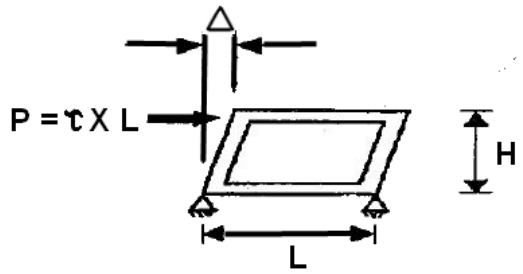

B. Flexural (Racking) Distortion of a Rectangular Frame

Figure 2.2 - General rectangular case for evaluating the flexibility ratio: distortions of a) free-field soil element and b) rectangular frame (from J.J. Wang, 1993)

Based on the results from dynamic analyses of buried rectangular structures, J.J.

Wang (1993) recognized a relationship between the flexibility ratio and the racking coefficient, $\mathrm{R}$ (Figure 2.3). The racking coefficient is the relative racking deformation of the structure with respect to the free-field ground deformation in simple shear and is given as:

$$
R=\frac{\Delta_{s}}{\Delta_{f f}}=\frac{\frac{\Delta_{s}}{H}}{\frac{\Delta_{f f}}{H}}=\frac{\gamma_{s}}{\gamma_{f f}}
$$

where $\gamma_{s}$ and $\gamma_{f f}$ are structural and free-field angular distortion and $\Delta_{s}$ and $\Delta_{f f}$ are structural and free-field lateral racking deformations respectively (Hashash et al. 2001). 
Results indicated that the flexibility ratio has the most significant influence on the normalized lateral racking deformations. Structural geometry and input motions were found to have little influence on the structural response. Varying embedment depth in the study showed that the normalized racking deflections decreased at very shallow depths. Some important conclusions regarding the flexibility ratio can be drawn from the study. For a flexibility ratio equal to 1.0 , the structure and soil are considered to have the same stiffness, implying that lateral deformation of the structure would be comparable to that of the free field soil. As the flexibility ratio approaches zero, the structure will not deform regardless of the distortion of the soil. When the flexibility ratio is greater than 1.0, the structure may distort more than the free-field soil. This phenomenon is not due dynamic amplification of ground motions. Instead, the inclusion of a structure creates a cavity or perforation in the surrounding medium, and the perforated ground naturally has reduced resistance to shear deformation than the free-field ground (J. J. Wang 1993).

The relationship in Figure 2.3 can be used as a practical design aid for estimating lateral racking deflections in buried rectangular structures. It provides an effective tool that goes beyond the free-field design approach by accounting for USSSI effects. It is important to note that racking deflection is given as normalized deflection; therefore, an understanding of the level of free-field deformation is needed. For example, even if the racking ratio is high, it could mean that the surrounding ground is very stiff, which implies very little free-field deformation. On the other hand, a high racking ratio may also imply a very flexible structure capable of safely accommodating high levels of distortion (J. J. Wang 1993). 


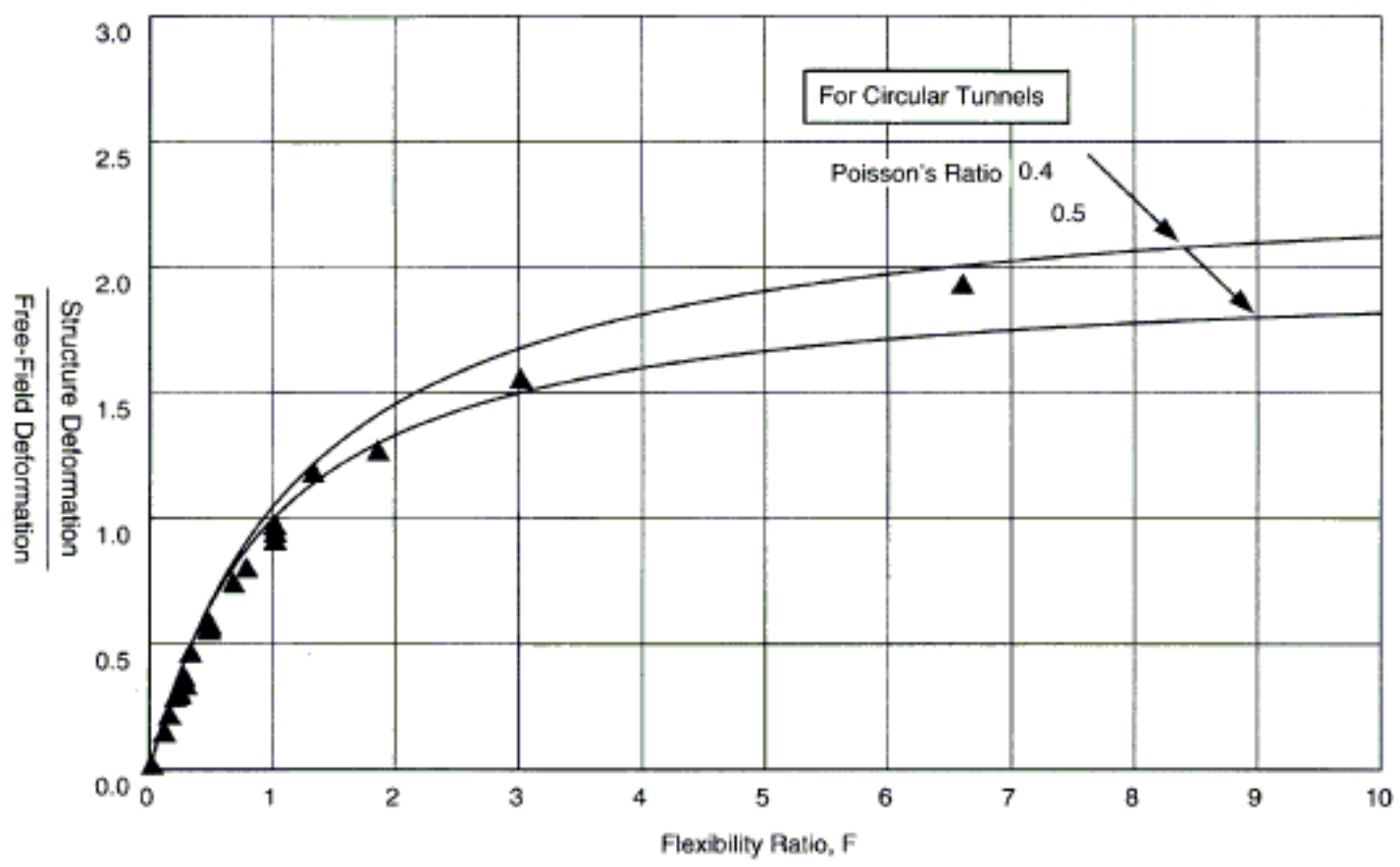

Filled Triangular Symbols: For Rectangular Tunnels Solid Lines: For Circular Tunnels

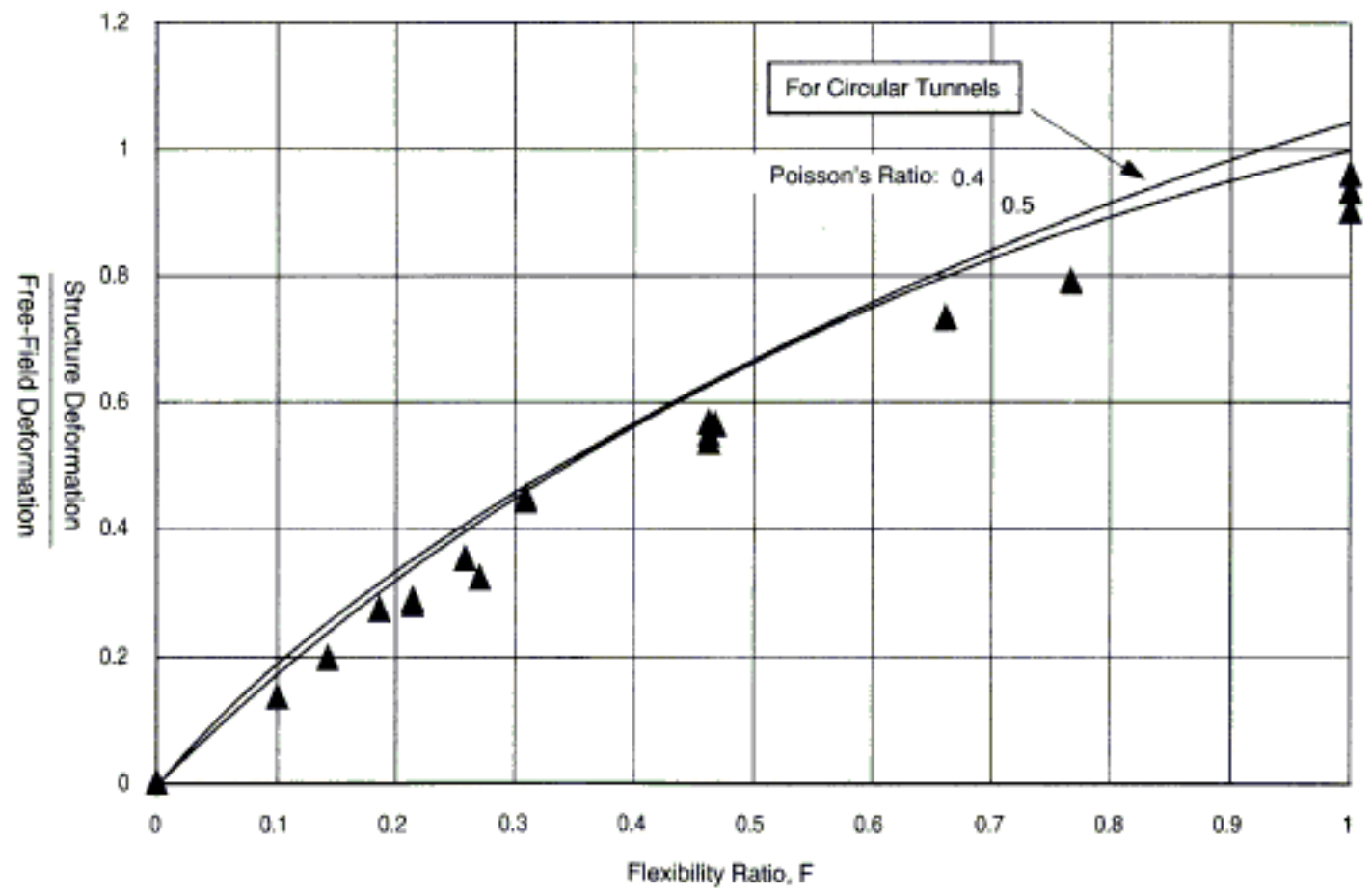

Filled Triangular Symbols: For Rectangular Tunnels Solid Lines: For Circular Tunnels

Figure 2.3 - Normalized structural deflections shown for rectangular structural linings superimposed with closed form solutions for circular linings (from Hashash et al. 2001) 


\subsubsection{Simplified Frame Analysis}

A simplified design procedure based on the conclusions from finite element analysis models is developed and presented by J.J. Wang (1993) for evaluating racking distortions of underground facilities. USSSI effects are considered through the relationship between the flexibility ratio and the racking ratio. It is a simple step-by-step procedure, which had been validated through comparisons with the soil structure interaction method. The method involves estimated free-field deformations just as in the free-field deformation method, and using the relationship shown in Figure 2.3 to estimate structural deformations. The structural deformations due to racking can then be imposed upon the frame, and member forces estimated using a structural analysis program. This method is advantageous, because it avoids the need for complicated finite element analysis, while still considers USSSI effects based on validated dynamic analysis. The full procedure can be referenced in J.J. Wang (1993) or Hashash et al. (2001).

\subsubsection{Analytical Models}

Penzien (2000) proposed an analytical model for estimating racking deflections of rectangular structures, which is in agreement with dynamic modeling results of Wang's study. This 2-dimensional plane-strain solution involves the relationship between a rectangular cavity being strained under free-field stress conditions and a similar cavity void of the applied free-field stresses. In this manner, the author postulates that rigid body rotation accompanies the racking deformations. Quantifying this rotation is not important in the analysis procedure. This method is simple and useful, because all that is needed for its application is knowledge of the Poisson's ratio of the free-field medium, 
the flexibility ratio, and free-field deformations due to vertically propagating shear waves. The model is considered approximate, since it does not account for normal stresses imposed by surrounding soil on the structure, but it gives a good approximation of soil-structure interaction effects. This is because normal stresses are considered secondary in transverse racking analyses.

With the aim of addressing the shortcomings of previous analytical procedures, Huo et al. (2006) introduced a complex analytical procedure using a 2-dimensional, plane-strain, pseudo-static analytical framework. Unlike Penzien's analytical procedure, the pseudo-static normal forces imparted on the structure are considered, in addition to the shear stress at the interface. The normal stresses acting on the structure are approximated using a linear distribution, which the author's claim to be "consistent with the symmetry of the problem" (Huo et al. 2006). True dynamic stress distributions are complex and cannot be readily characterized, but approximation of normal stress distributions under pseudo-static conditions may be more appropriate than disregarding them altogether. Shear stresses are considered in a similar fashion as previous research in that they are modeled as a uniform stress distribution acting on all sides of the structure. Analytical results are validated and calibrated through pseudo-static finite element procedures, and comparisons are made with previous studies (Figure 2.4). It is clear that for flexibility ratios greater than 1.0 , the racking ratio increases more dramatically than results from Penzien and Wang. If the flexibility ratio is less than 1.0, common for rectangular cut-and-cover sections, results are similar to those of previous studies. This study finds that the aspect ratio of the structure affects the level of racking deformation, which is somewhat contrary to earlier conclusions. The aspect ratio is quantified by the 
dimensionless shape factor $(\lambda)$, which is the width divided by the height of the structural opening. Although the analytical solution presented in Huo et al. (2006) is complex on paper, it is a practical solution that can be readily integrated into spreadsheet form.

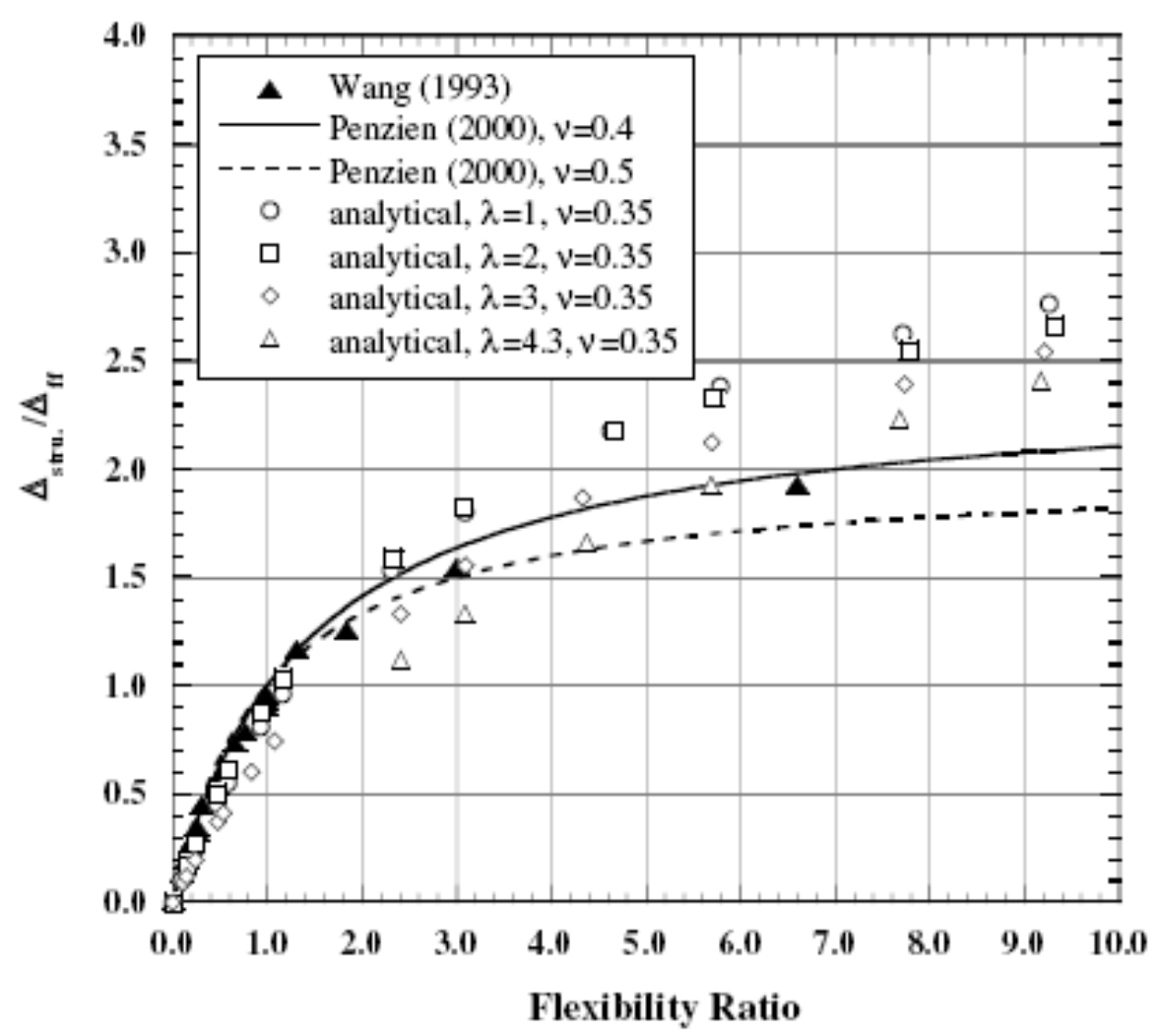

Figure 2.4 - Normalized racking deformations with respect to the flexibility ratio from multiple researchers (from Huo et al. 2006)

Analytical approaches are not without fundamental limitations. These limitations must be understood and considered by the design engineer before use. In both methods discussed here, the structure is assumed to be sufficiently deep for surface boundary effects to be considered negligible. According to J.J. Wang (1993), this is when the depth of the soil cover is greater than or equal to the height of the structure. Another limiting assumption for the analytical methods is that the medium is modeled as an elastic half-space and is homogeneous and isotropic. Despite their limitations, these analytical 
approaches provide a simple effective means for estimating racking deformations. If not used as primary design tools, analytical solutions can be used as checks for other current or developing design methods and research studies.

\subsubsection{Numerical Methods}

Hashash et al. (2001) explains that seismic soil-structure interaction is a complex problem "that may require the use of numerical methods" and that "this is especially true for cut-and-cover structures because of their greater vulnerability to seismic damage.” Also, numerical analysis may be needed in cases where the seismic response of complex or non-uniform shapes cannot be accurately characterized by closed form solutions. This type of rigorous analysis can involve complex three-dimensional (3D) USSSI effects or simpler 2D plane-strain equivalent linear problems. A full detailed discussion of numerical methods is beyond the scope of this paper, but the following sections include numerous examples where numerical methods have been used to evaluate field observations and validate lab testing results. Numerical methods can also be employed to evaluate the seismic capacity of existing structures which may or may not have been built using seismic design principles. An example of this is the use of 3D numerical analysis to evaluate the seismic capacity of the Alameda tube tunnels which connect Oakland, California to Alameda Island (Kozak et al. 1999). Dynamic analysis methods can also be used in conjunction with experimental testing to further validate and expand on previous works regarding USSSI effects including horizontal racking. 


\subsection{Case Studies of Damage to Tunnels}

A limited number of cases exist where there has been severe structural damage to underground facilities during earthquakes. As a result, there is confidence that underground facilities are safer than above ground facilities under seismic loading (Huo et al. 2006). Although this may typically be the case, severe damage to underground structures is still a significant concern. The following provides a discussion of such cases where severe damage has resulted from intense ground shaking.

\subsubsection{Collapse of Daikai Subway Station in Japan}

The 6.9 moment magnitude Kobe earthquake, otherwise known as the HyogokenNanbu earthquake or the Great Hanshin earthquake, hit the Kobe and Osaka region of southwestern Japan on January 17, 1995. It was the most destructive earthquake to strike Japan since the Great Kanto earthquake of 1923. The earthquake resulted in more than 5,500 deaths, damage to more than 200,000 homes, economic loss upwards of 200 billion dollars, and severe damage to critical infrastructure (Meymand 1998). The collapse of the Daikai subway station was one of the most surprising examples of damage during the earthquake due to the structure's location underground.

The Daikai subway station, part of the privately owned Kobe Rapid Transit System of Kobe City, represents the most recognized and widely studied example of failure of an underground facility due to the unusual circumstances surrounding its collapse. Huo et al. (2004) explains the station's importance as an area of study, because "it is the first underground structure not crossing an active fault that has completely collapsed during an earthquake without liquefaction of the surrounding soil." It is also an 
important case because the station contained multiple geometrically different sections that behaved differently. The station was built using cut-and-cover methods and is divided into three structural sections: a widened central section containing the passenger loading platforms, the subway tunnels, and a station access section with an upper level. Iida et al. (1996) presents observations from detailed reconnaissance study that was performed shortly after the earthquake. The paper provides a detailed description of the soil conditions, which can be summarized as transitioning from silty/clayey soil west of the site to more sandy soil east of the site overlying a stiffer base layer. Further geological data is available in more recent papers (Huo et al. 2004, 2005; ParraMontesinos et al. 2006). Near-field backfill for the structure was an engineered fill found to have SPT blow counts of about 10 blows per foot (bpf) for almost the entire depth (except near the bottom) implying relatively weak near-field soil conditions. It was observed that collapse of the ceiling slab occurred due to the complete failure of 23 reinforced concrete columns in the central section of the station (Figure 2.5 and Figure 2.7). Columns in the adjoining structural sections suffered less severe damage and did not collapse. The road and backfill directly above the approximately 90 meter length collapsed section experienced subsidence of up to more than 2.5 meters (Figure 2.6 and Figure 2.7). In addition to collapse, significant cracking was observed in both longitudinal and transverse directions (Iida et al. 1996). 


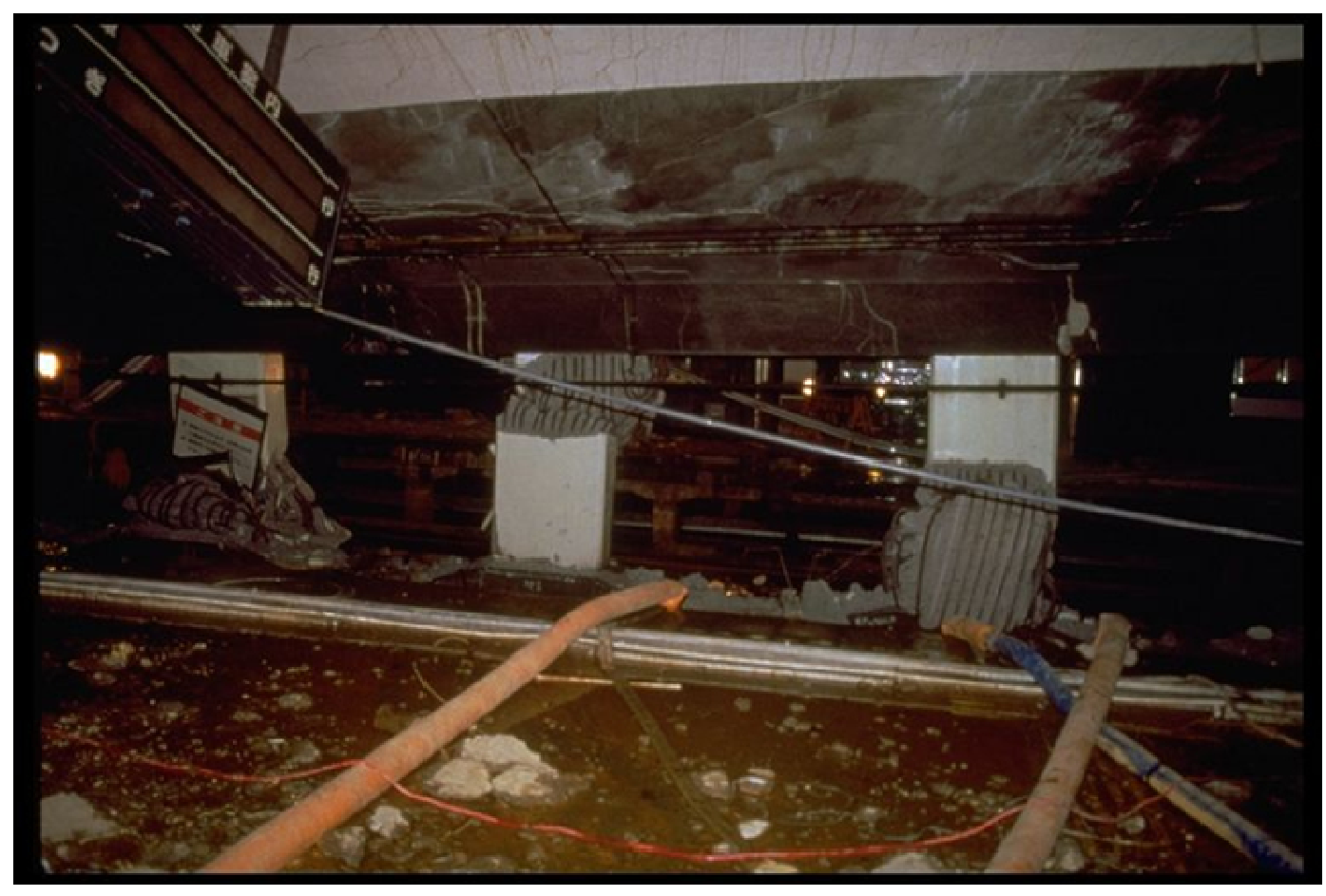

Figure 2.5 Damage to unconfined interior columns, leading to roof slab collapse in the Daikai subway station (from Sitar 1995)

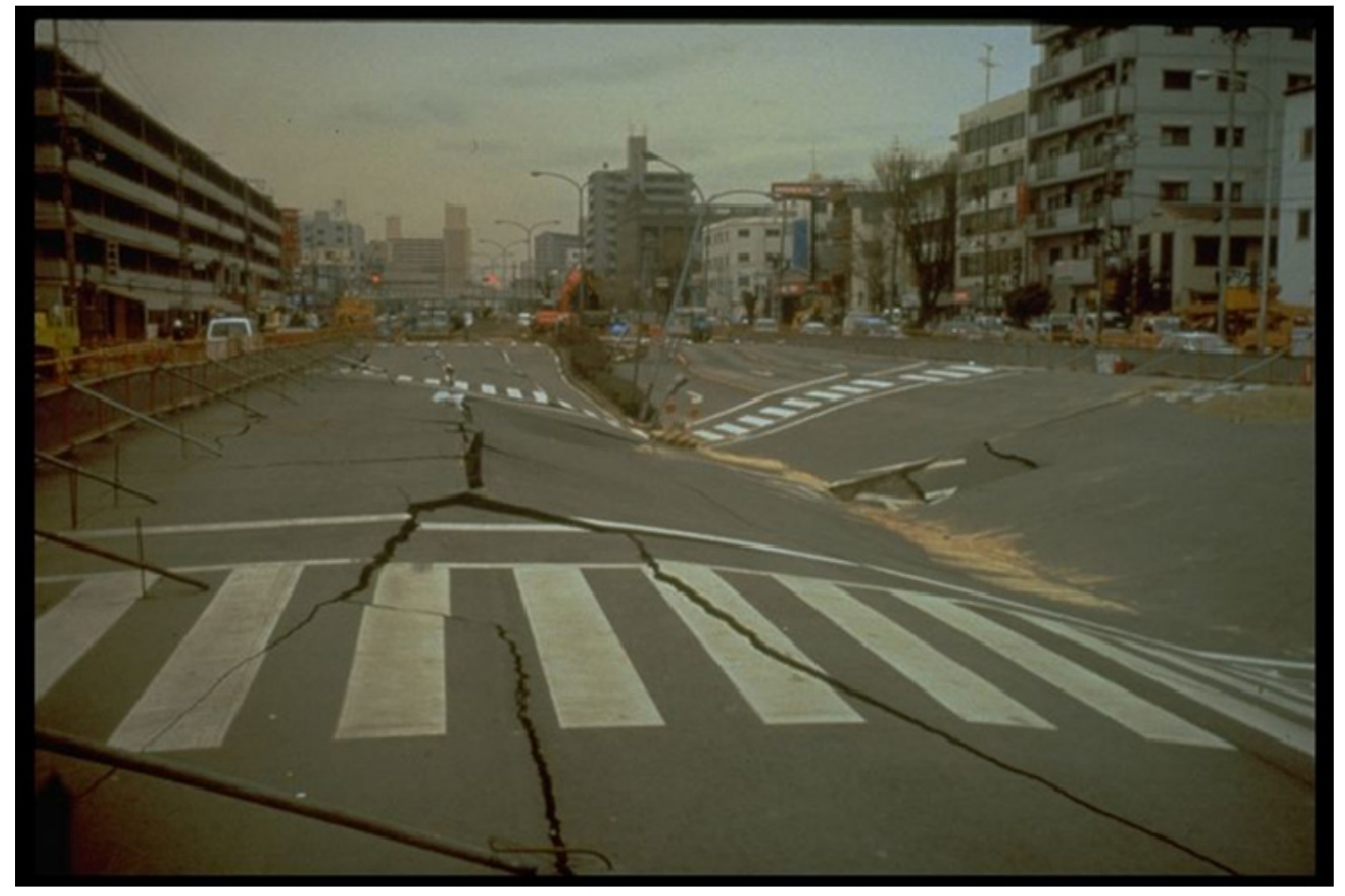

Figure 2.6 - Ground subsidence on road above the Daikai station (from Sitar 1995) 


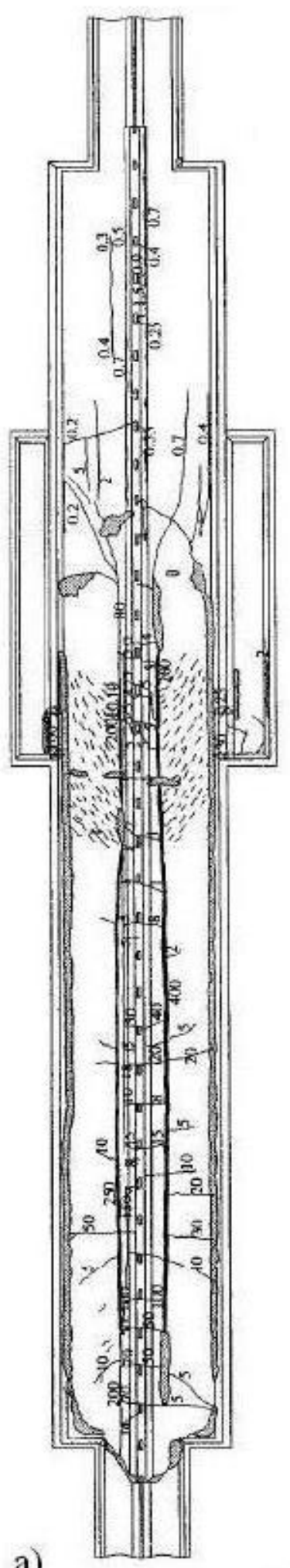

a)

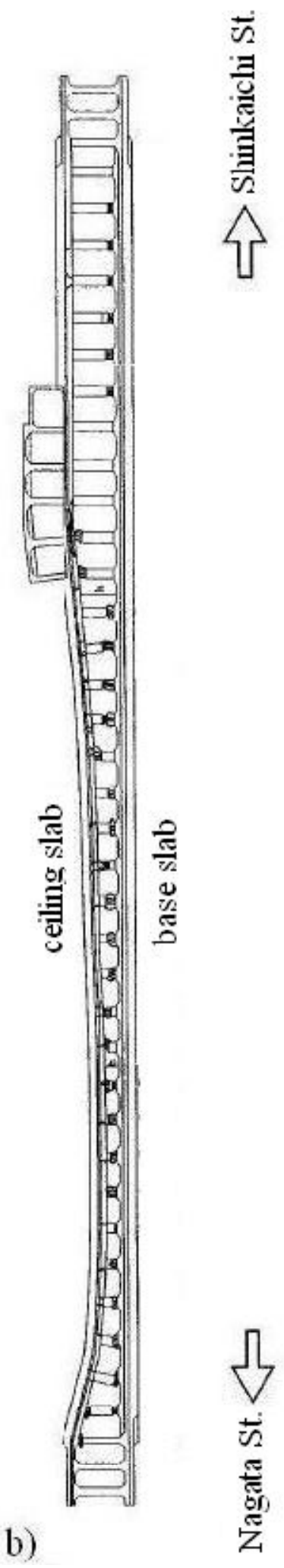

Figure 2.7 - Damage patterns observed in the Daikai station collapse showing a) crack patterns in the interior ceiling slab (small numbers are crack width in mm) and b) elevation view of collapsed columns and ceiling slab (after Iida et al. 1996) 
The failure mechanism of the central section of the station has been a subject of some debate; however it is widely recognized that the central columns were not designed to sufficiently resist the lateral earthquake deformations (Huo et al. 2004). One early study surmised that failure of the columns occurred under a combination of shear loading and moment due to eccentric axial load resulting from excess lateral displacement (Iida et al. 1996). Another study argued that shear failure due to strong horizontal ground motion resulted in loss of axial load carrying capacity, and that vertical motions had little influence (An et al. 1997). Research in recent years concerning the Daikai station has focused on using sophisticated finite element analysis to gain further understanding of USSSI effects for cut-and-cover structures (Huo et al. 2004; Parra-Montesinos et al. 2006). Both of these studies were performed using the general finite element analysis program ABAQUS (Hibbitt and et al. 2001). The author's of the papers cite its advantages in that it allows for users to define custom material models and interface behavior between material contacts.

Huo et al. (2004) conducted simulations considering hysteretic elasto-plastic behavior to account for nonlinear behavior of soils in response to cyclic loading and unloading. The purpose of the simulations was to evaluate the load transfer mechanism between the subway station and the surrounding ground. Results of the study support the conclusion that the failure of the columns was due to drift of the columns under racking deformations imposed onto the structure by the ground. The columns were not design adequately to accommodate the large drift demands leading to shear failure and subsequent loss of axial load capacity and collapse of the ceiling slab. Important findings in the study are best described by the following excerpts: "unconfined structural members 
within underground structures (i.e. free-standing columns within the structure) behave under seismic loading as regular columns in an above-ground structure," and "the main difference between free-standing columns above and below the ground surface is that the drift in the underground structure columns is controlled by the stiffness of the degraded surrounding ground, while in the columns above ground, the drift is entirely determined by the stiffness of the structure" (Huo et al. 2004). This is important because it provides understanding of the behavior of the interior of underground structures in terms of well understood concepts relating to surface structures. The concept that the seismic behavior of underground structures is mostly controlled by deformation is reinforced by this research, while suggesting that design philosophies usually reserved for surface structures may be employed in appropriate situations.

An extension of the previous study, Huo et al. (2005) discusses in more detail the load transfer mechanisms between the structure and the surrounding ground. Findings suggest that for relatively stiffer structures, shear modulus degradation of the surrounding ground is limited when compared to that of the free-field, thus leading to higher confinement and lower structural deformation. The author's describe this phenomenon as a result of stiffer sections having a large "attached" stiff soil mass, helping to limit deformations. Dynamic numerical simulations support this conclusion along with the empirical observation that the collapsed central section had a lower relative stiffness than the other un-collapsed sections. Another important finding is that the interface friction between the structure and the soil has a significant effect on dynamic load transfer. Results indicate that the highest column deformation for the Daikai station occurs using an interface friction coefficient $\mu=0.4$. This value is between the two idealized cases of 
the frictionless (full-slip) condition and perfect attachment (no-slip), which are usually assumed in analytical models. Numerical simulations illustrate the effect of interface friction on near-field modulus degradation, shown in Figure 2.8 for a) full slip and b) noslip. The authors stress that the numerical conclusions regarding the Daikai subway station cannot be generalized for other structures due to variability in structural shape, depth, ground conditions, motions, and any other pertinent factors.
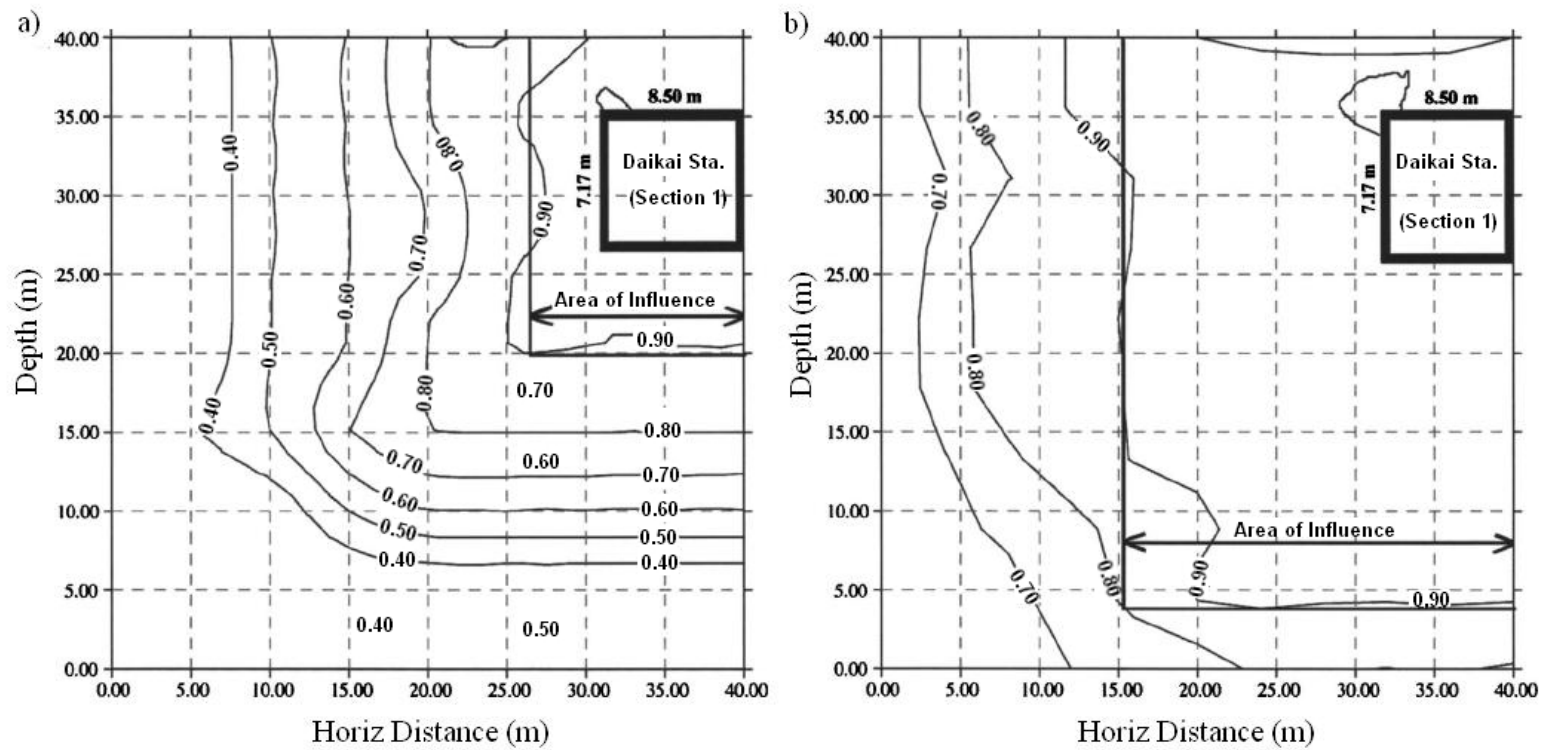

Figure 2.8 - Soil-structure interface friction effects on soil modulus degradation for a) noslip condition and b) full-slip condition where contours are $\mathrm{G} / \mathrm{G}_{\max }$ (after Huo et al. 2005)

Further numerical investigation of the failure of the Daikai subway station is provided by Parra-Montesinos et al. (2006), in which the study goes a step further by running a second numerical model on the structural column itself. A primary aim of the study was to estimate the likelihood of structural failure under the predicted drift demands. Models showed results consistent with the damage observed in the field and provided evidence that the high axial load due to soil overburden contributed to collapse 
of the structure by reducing the drift capacity of the columns. This echoes the early conclusions by a field investigation which surmised that the failure of the columns was due to a combination of vertical and shear loading during the earthquake (Sitar 1995).

The Daikai station is the most important case study regarding to damage to shallow cut-and-cover structures to date because it represents a large-scale experiment for studying USSSI effects. Observations of the failure have allowed effective use of numerical modeling, leading to important conclusions regarding the behavior of shallow cut-and-cover structures. The behavior of the Daikai subway station helps to validate the assertion that a critical factor concerning dynamic underground structural response is the relative stiffness between the structure and the surrounding medium. Other important conclusions are drawn from the case study regarding soil-structure load transfer mechanisms and structural design considerations for interior structural elements.

\subsubsection{Damage to Mountain Tunnels}

There have been many recent studies on the seismic performance of mountain tunnels during earthquakes (J. H. Hwang and Lu 2007; Lu and J. H. Hwang 2008; W. L. Wang et al. 2001; Z. Z. Wang et al. 2009). A majority of these studies express that underground facilities have historically experienced less damage than above ground structures. Field observations shed light on possible damage patterns for mountain tunnels, which should not be overlooked. The following provides a comprehensive overview these damage patterns which have resulted from significant seismic events.

A comprehensive assessment of damage to mountain tunnels due to the September 21, 1999 Chi-Chi earthquake in Taiwan is presented by W.L. Wang et al. 
(2001). Fifty-seven mountain tunnels were investigated after the earthquake using methods ranging from visual inspection to non-destructive methods, such as ground penetration radar. Of the 57 tunnels investigated, 8 were considered totally undamaged, 26 were considered lightly damaged, 11 were considered moderately damaged, and 13 were considered severely damaged. This classification is adopted from criteria presented by Huang et al. (1999), which considers the serviceability of a tunnel after an earthquake. Slightly damaged tunnels can run under normal operation, moderately damaged tunnels can operate with traffic restrictions, and severely damaged tunnels cannot operate at all. Their summary table of all 57 cases provides a concise and detailed review of the investigation. Tunnels found in the hanging wall east of the Chelungpu thrust fault line suffered most of the serious damage compared to tunnels in the footwall (W. L. Wang et al. 2001).

Damage was characterized under the following nine distinct patterns: sheared off lining, slope failure induced tunnel collapse, longitudinal cracks, transverse cracks, inclined cracks, extended cross cracks, pavement or bottom cracks, wall deformation, and cracks nearby openings. As a result of these damage types, secondary effects were observed such as concrete spalling, blockage of tunnel portals (Figure 2.9), water leakage and flooding, exposed reinforcement, upheaval of roads, damage to lighting and ventilation systems, and partial or total disruption of traffic. Damage was found to be significant in lined tunnels in areas where the earth medium is relatively soft, overburden is relatively low, or where construction issues such as cave-in may have occurred prior. The researchers point out that "however badly the tunnels were damaged, they remained relatively unscathed compared to surface structures" (W. L. Wang et al. 2001). 


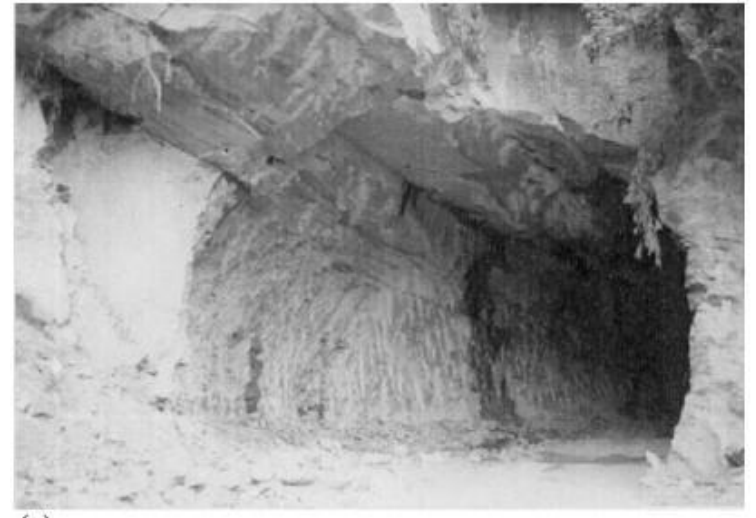

(a)

Figure 2.9 - Photograph of the Chi-Shue tunnel portal (a) before and (b) after the 1999 Chi-Chi earthquake (from W.L. Wang et al. 2001)

Hwang and $\mathrm{Lu}$ (2007) provide an assessment of the performance of the old Sanyi railway tunnels in Taiwan, built in 1908 and abandoned 90 years later after construction of the new Sanyi railway tunnel. These old tunnels have experienced two significant earthquakes in their lifetime, the 1935 Hsinchu-Taichung earthquake and the 1999 ChiChi earthquake. In the 1935 event, tunnel collapse did not occur despite severe damage to the original red brick lining. Significant damage occurred at the tunnel portals in the form of cracking and deformations (Figure 2.10). Most of the damaged tunnel sections were repaired and retrofitted with plain concrete in the years following the earthquake. The 1999 Chi-Chi earthquake tested the tunnels once again and this time damage was minimal. Cracking was apparent in three tunnels. It is not clear if the earthquake induced the cracking, because the tunnels had not recently been inspected before the earthquake. The researchers used damage observations of the old Sanyi tunnels to calibrate a method for evaluating seismic tunnel performance. The method is based on quasi-static finite difference analysis, and is termed by the authors as the Modified CrossSection Racking Deformation Method (MCSRD). Results of the numerical analysis 
agreed with damage observed in the field. Unlike a full dynamic analysis, it does not simulate non-linear and hysteretic behavior (J. H. Hwang and Lu 2007).

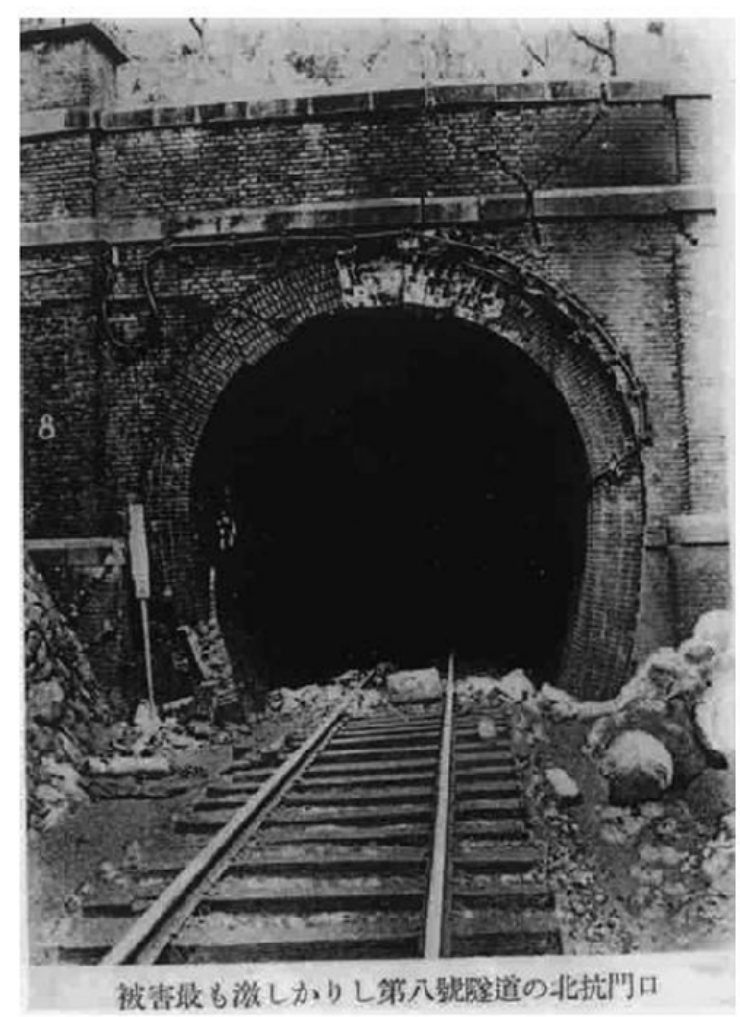

Figure 2.10 Damage to the old Sanyi No. 8 south portal after the 1935 Hsinchu-Taichung earthquake (from Hwang and $\mathrm{Lu}$ 2007)

In a subsequent study, Lu and Hwang (2008), observed damage to the new Sanyi railway tunnel in Taiwan after the 1999 Chi-Chi earthquake. The tunnel was a replacement for the old Sanyi railway tunnels previously discussed, and had been in operation for about a year when the earthquake struck. The tunnel was built using the economical NATM (New Austrian Tunneling Method) design criteria, which utilizes flexible support as a primary load bearing lining, and an unreinforced concrete secondary lining. The paper discusses five main issues leading to tunnel damage that were identified through field investigations, some of which are a result of using NATM. First, 
the second lining of the tunnel was not designed to withstand the large seismic loads imposed by the earthquake. Second, the irregular geometry of the tunnel lining wherever tunnel refuges existed resulted in damaging stress concentrations at corners during shaking. Third, imperfect backfill behind the lining resulted in voids and weak lining bonds that can lead to concrete spalling. Fourth, the lack of steel reinforcement in the second lining severely reduces shear and bending moment capacity under seismic loading compared to steel reinforced linings. Last, much of the damage occurred in geologically weak zones which are more susceptible to ground deformations. The previously mentioned MCSRD method was used to evaluate the damage mechanisms associated with the new tunnel at its most severely damaged section. The section, which contained large refuges, was built through highly fractured sandstone and shale and was found to contain voids behind the concrete lining. According to the authors, the MCSRD method was able to identify the failure mechanism of this section and can simulate harmful effects from all of the aforementioned damage factors. The method shows promise in the assessment of the seismic capacity of tunnels, but should be further validated as appropriate case studies become available.

Yashiro et al. (2007) provides a summary of historical damage to mountain tunnels in Japan for the 1923 Kanto, the 1978 Izu-Oshima-Kinkai, the 1995 HyogokenNanbu, and the 2004 Niigataken-Chuetsu earthquakes. Figure 2.11 summarizes the historical damage where earthquake magnitudes are given in Richter magnitude and supplemented with the Japan Meteorological Agency (JMA) seismic intensity scale. Discussed here are investigations regarding the most recent of the four earthquakes, the Niigataken-Chuetsu earthquake of October 23, 2004. 


\begin{tabular}{|l|c|c|l|}
\hline \multicolumn{1}{|c|}{ Year, Name } & $\begin{array}{c}\text { Magni| } \\
\text {-tude }\end{array}$ & $\begin{array}{l}\text { Seismic } \\
\text { intensity }\end{array}$ & \multicolumn{1}{|c|}{ Tunnel performance } \\
\hline $\begin{array}{l}1923 \\
\text { Kanto }\end{array}$ & 7.9 & 6 & $\begin{array}{l}\text { Damage to 93 tunnels } \\
25 \text { tunnels required reinforcement }\end{array}$ \\
\hline $\begin{array}{l}1978 \\
\text { Izu-Oshima-Kinkai }\end{array}$ & 7.0 & 5 & $\begin{array}{l}\text { Damage to 9 tunnels } \\
\text { 2 tunnels required reinforcement }\end{array}$ \\
\hline $\begin{array}{l}1995 \\
\text { Hyogoken-Nanbu }\end{array}$ & 7.2 & 7 & $\begin{array}{l}\text { Damage to 7 tunnels } \\
5 \text { tunnels required reinforcement }\end{array}$ \\
\hline $\begin{array}{l}2004 \\
\text { Niigataken-Chuetsu }\end{array}$ & 6.8 & 7 & $\begin{array}{l}\text { Damage to 24 tunnels } \\
5 \text { tunnels required reinforcement }\end{array}$ \\
\hline
\end{tabular}

Figure 2.11 - Summary of damage to mountain tunnels in Japan during major earthquakes (from Yashiro et al. 2007)

Three special damage patterns are identified as Type I, II, and III. Type I is damage to shallow tunnels (Figure 2.12a), which is characteristic of cracks in the arch of the lining caused by transverse displacement (racking). This is reminiscent of the behavior of cut-and-cover rectangular tunnels in shallow conditions. Type II is damage to tunnels founded in poor geological conditions, such as highly fractured rock zones (Figure 2.12b). In these situations, squeezing pressures on the tunnel can be increased causing severe damage to tunnel linings. Type III is damage to tunnels by fault sliding (Figure 2.12c). This occurs where a tunnel crosses a fault that experiences offset during the earthquake resulting in cracking patterns associated with various forms of stresses (i.e. tensile, compression, and shear). Other special conditions exist. For example, the lining may contain existing structural defects. Two conditions affecting damage risk were considered: 1) earthquake magnitude and 2) tunnel location relative to the epicenter or fault rupture. A relationship between these two factors are drawn which show that for higher magnitude earthquakes, damaged tunnels can be observed farther from the fault 
rupture. This relationship does not consider the aforementioned special conditions. The authors concluded that the damage risk is highest when the earthquakes are large and the tunnels are close to the fault rupture, and that the level of damage observed is largest if one or more of the special conditions exist (Yashiro et al. 2007).

a) I. Shallow tunnel

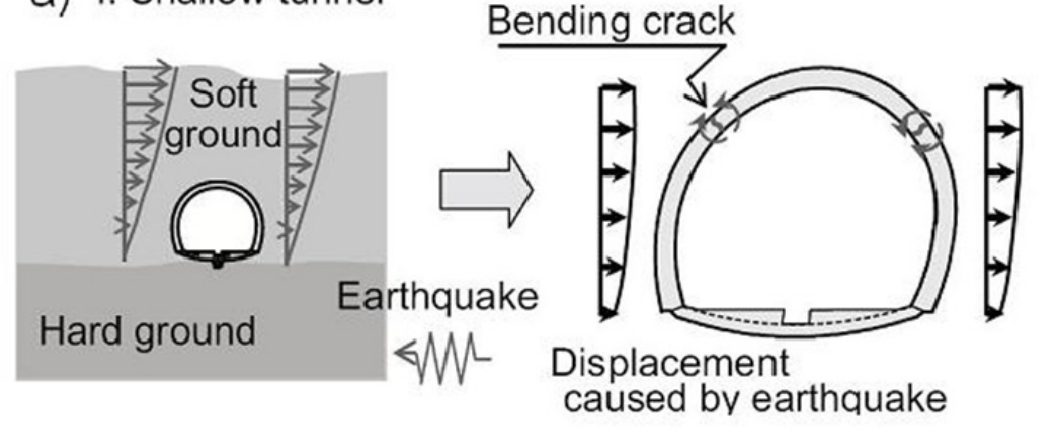

b) II. Poor geological conditions

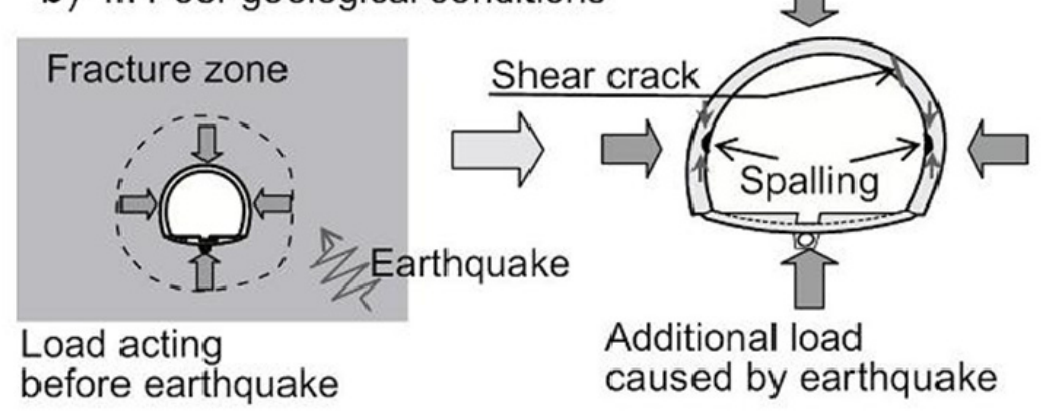

C) III. Fault slide

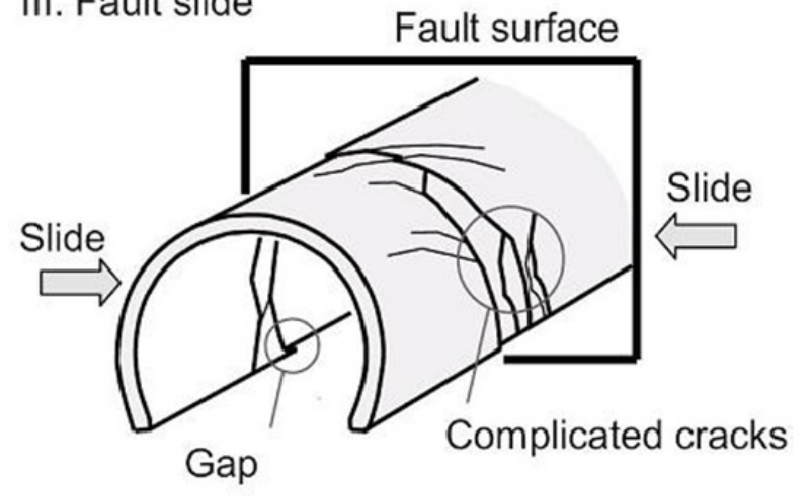

Figure 2.12 - Damage patterns observed in mountain tunnels (after Yashiro et al. 2007) 
Z. Z. Wang et al. (2009) provides the results of an investigation into damage of mountain tunnels in China resulting from the magnitude $7.9\left(\mathrm{M}_{\mathrm{w}}\right)$ Wenchuan earthquake of May 12, 2008. The investigation focused on the Du Wen Highway which contains 18 tunnels that experienced damage. Based on the tunnel operation classifications presented earlier, one tunnel was considered lightly damaged, 4 tunnels were considered moderately damaged, and 13 were considered severely damaged. Damage patterns were similar to those found during investigations of tunnels in Taiwan and Japan as previously discussed. Portal failure was the most widespread of all the damage types encountered, affecting 15 of the 18 tunnels investigated. Shear failure of the lining due to fault displacement was another major consequence of the earthquake, affecting half of the tunnels. Other damage encountered included rockfalls, pavement cracking, concrete lining spalling, lining cracks, and water leakage. Recommendations stressed by this particular research are the importance of designing tunnels and tunnel portals farther from slope faces to reduce damage from slope failure and to avoid planning tunnel alignments across active fault surfaces.

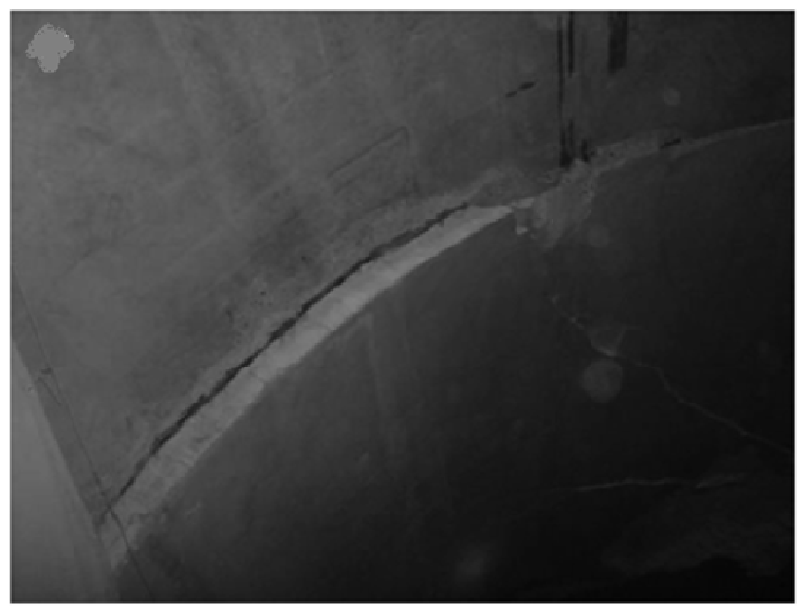

Figure 2.13 - Shearing of Longchi tunnel lining due to fault displacement (from Z. Z. Wang et al. 2009) 
Based on the reviewed literature regarding mountain tunnels, the following observations can be made.

- Mountain tunnels are often founded in competent material such as rock, consequently making them less susceptible to large distortions. When founded in weak geologic conditions, the lining are susceptible to serious damage such as concrete spalling, cracking, and possible lining collapse. Lining collapse does not necessarily lead to collapse of the in situ material into the tunnel cavity.

- One of the most serious concerns regarding mountain tunnels is damage to tunnel portals. This can include cracking of portal structures and blockage of portals due to slope failure. In the latter case, the tunnel can lose all operational capacity until appropriate repairs are made.

- Tunnels crossing active fault planes are highly vulnerable to liner shearing due to fault offset.

- Construction methods and construction quality can have a significant impact on the performance of mountain tunnels under seismic loading.

A universal opinion expressed by the mountain tunnel researchers is that that more data is needed regarding the seismic performance. These researchers also stress the importance of developing methods for evaluating the performance of mountain tunnels under seismic loading. Hwang and Lu (2007) attempted to address this concern with the development of the MCSRD method, which compared well with field observations of the old and new Sanyi railway tunnels in Taiwan. To date, the procedure does not appear to be validated by further research. 


\subsubsection{Damage to the Bolu Highway Twin Tunnels}

Kontoe et al. (2008) explains that construction began on the Bolu highway twin tunnels in Turkey in 1993 and 1994 at the Elmalik (west) and Asarsuyu (east) tunnels respectively. The tunnels were constructed in four drives, two from the Asarsuyu portal and two from the Elmalik portal using the NATM tunneling method, which was later deemed unsatisfactory in poor ground conditions. In 1999, two major earthquakes hit the Bolu region in a period of three months. The first was the magnitude $\left(\mathrm{M}_{\mathrm{w}}\right) 7.4$ Kocaeli earthquake of August 17, 1999, during which the tunnels suffered no damage save for hairline cracks in the lining of completed tunnel sections. These cracks were monitored in the weeks following and no discernable movement was detected (Hashash 2001). On November 12, 1999 the magnitude $\left(\mathrm{M}_{\mathrm{w}}\right)$ 7.2 Duzce Earthquake struck, which caused major damage in both drive sections (Amberg and Russo 2001). Research by Kontoe et al. (2008) suggests that the disparity in the performance of the tunnels during the two events was a consequence of the distance to fault rupture. The epicenter of the Duzce earthquake was within $20 \mathrm{~km}$ of the western tunnel portals and the eastern tip of the fault rupture was only about $3 \mathrm{~km}$ distance from the same point. This is comparatively much closer than the Kocaeli earthquake, in which the closest point of the fault rupture was approximately $30 \mathrm{~km}$ to the west. Since the Bolu tunnel did not cross the fault rupture, it is unlikely that damage occurred due to fault offset. However, due to its close proximity to the fault rupture, it is presumed that near fault effects played a significant role in the ground motions experienced at the tunnel locations.

According to Hashash (2001), the most severe damage occurred in unfinished sections of both Elmalik drives beginning about 300 meters from the tunnel portal. It is 
important to note that the tunnel was finished for the first 300 meters and experienced little damage under the seismic loading. The unfinished section was aligned though fault gouge clay and had been built according to NATM; therefore, it was only lined with shotcrete and reinforced with bolt anchors at the time of the earthquake. Dalgıç (2002) reports, based on investigative drill holes, that the shotcrete lining either collapsed or completely deformed over an approximately $400 \mathrm{~m}$ section. The collapse was found to be progressive and appeared to cause two sink holes at the surface. The first was an immediate consequence of the earthquakes, and the second appeared two months afterward due to progressive collapse, partly influenced by aftershocks. Appearance of sinkholes suggests that increased lateral earth pressures on the sidewalls of the tunnel during the earthquake influenced collapse. Citing a study by O'Rourke et al. (2001), Hashash (2001) suggests collapse mechanisms such as strong ground motions, displacement across fault gouge material, and landslides. The first mechanism is in agreement with the assessment that increased lateral earth pressures may have led to collapse. There has been an overall lack of analysis regarding the collapse mechanism of the eastern tunnel sections, because they were unfinished at the time of the earthquake and not expected to fully withstand such a large seismic event. The adjacent finished section performed well during the same event.

The Asarsuyu (western) tunnel drives also sustained damage during the Duzce earthquake. Similarly to the Elmalik (eastern) side, it was the unfinished portions of the tunnels that sustained the significant damage. According to Kontoe et al. (2008), these tunnel portions were excavated through the worst ground conditions along the alignment, which was an extensive zone of uniform highly plastic fault gouge clay. Sections built in 
this poor ground using the NATM method would experience large, uncontrollable static deformations as a result of ground squeezing. Thus promoting the use of a heavy construction method which consisted of excavating two 5.6-m diameter bench pilot tunnels (BPT) at a 19-m center-to-center spacing, then supported by circular steel ribs at 1.1 meter longitudinal spacing and lined with $30 \mathrm{~mm}$ thick shotcrete. Finally, the BPT's would be backfilled with concrete for use as abutment for the main tunnel linings. A schematic of this heavy tunnel section is shown in Figure 2.14.

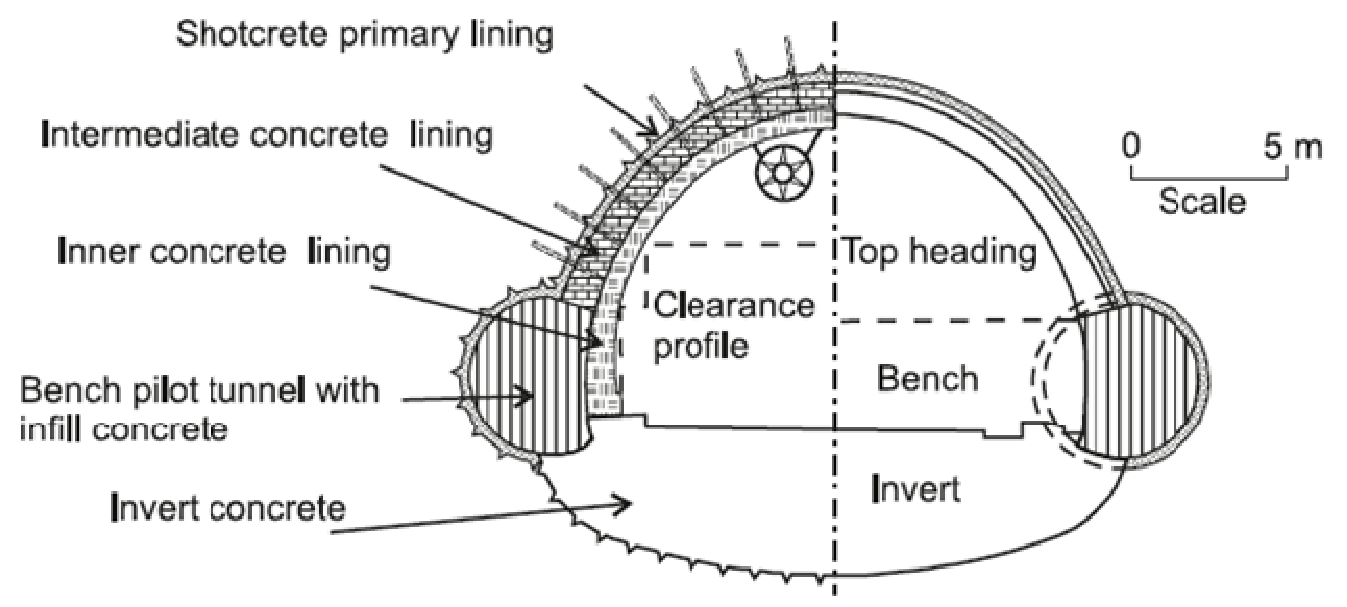

Figure 2.14 - Schematic of the tunnel cross-section though fault gouge clay, showing backfilled pilot holes and main tunnel linings (from Kontoe et al. 2008)

Kontoe et al. (2008) focuses on collapse and tunnel deformation, which occurred over a length of about $30 \mathrm{~m}$ in both the left and right bench pilot tunnels of the left Asarsuyu drive (herein called LBPT and RBPT). The tunnels were newly excavated and had not yet been backfilled with concrete. The LBPT drive was staggered ahead of the RBPT drive within the fault gouge, and interestingly, only portions of the tunnels that overlapped longitudinally experienced collapse. Full 2D dynamic finite element analyses 
were performed to study the USSSI response of the BPTs. The study used the strong ground motion recorded at the Bolu station approximately $18.3 \mathrm{~km}$ from the fault surface rupture, which has a peak ground acceleration of $0.81 \mathrm{~g}$. As per FHWA recommendations, the motion was scaled by a factor of 0.7 to account for attenuation with depth and truncated to capture the important shaking content. The scaled motion used in the analyses is shown in Figure 2.15.

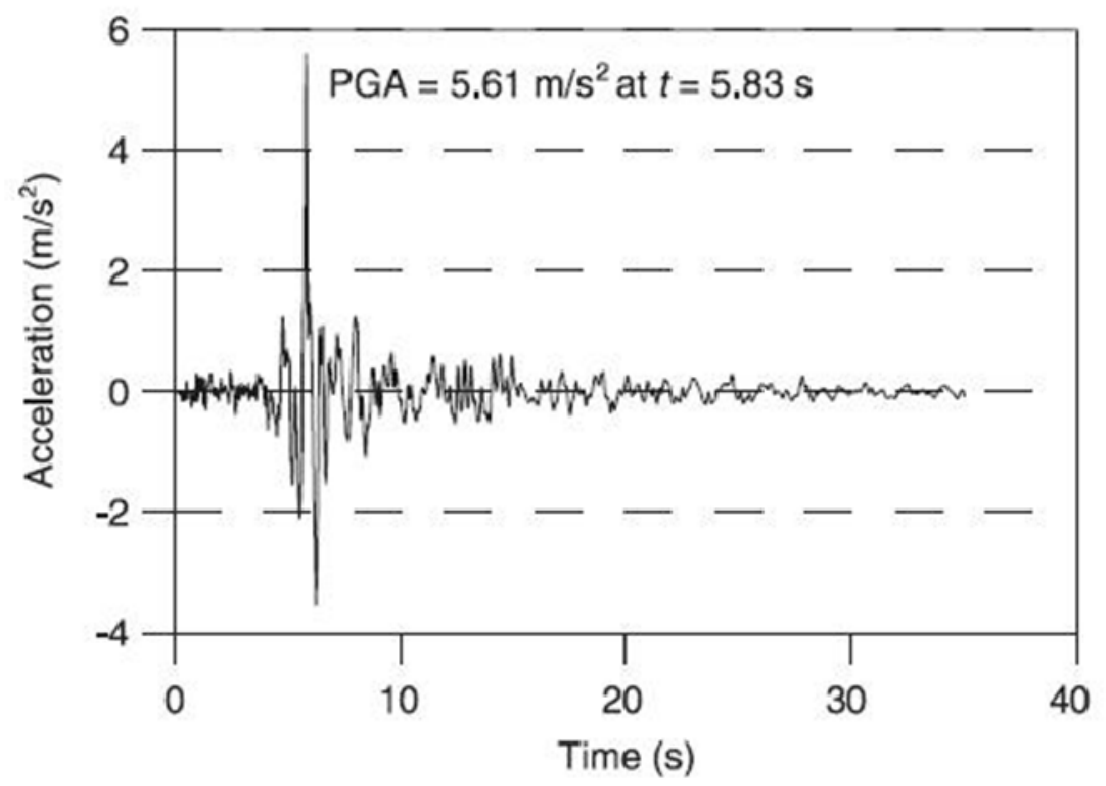

Figure 2.15 - Accelerogram used in 2D finite element analysis (from Kontoe et al. 2008)

The first analysis was performed for the section where both BPTs drives overlapped and collapsed, and the second analysis was run for the section where the uncollapsed LBPT was driving ahead of the RBPT. In addition to understanding the failure mechanism of the tunnels, the study hoped to address the issue of why collapse only occurred where the pilot tunnels overlapped. Two explanations were identified. First, the relatively narrow soil pillar between the tunnels could have caused wave reflections 
and amplified ground motions within the pillar. And second, the different stratigraphy of the sections could have affected the seismic loading on the tunnels. Results of the study indicated that the presence of a soil pillar between the tunnels had only a minor effect on tunnel response, and that stratigraphy had a more pronounced effect on seismic performance. The $2 \mathrm{D}$ dynamic racking analysis estimated seismic loads greater than the expected strength of the shotcrete in the mostly undamaged section. The authors recommend that complex $3 \mathrm{D}$ dynamic analysis may be required to explain these findings (Kontoe et al. 2008).

Analysis suggested that the tunnels deformed in an oval shape, which is in agreement with the deformation mechanism proposed by earlier researchers for horizontal racking of circular tunnels (Hashash et al. 2001, 2005; Penzien 2000; Penzien and Wu 1998; J. J. Wang 1993). It is also in agreement with damage patterns observed in the LBPT tunnel. Results of the 2D finite element analyses were compared with quasistatic methods and analytical elastic solutions for circular tunnels by J.J. Wang (1993) and Penzien (2000). The study found that both elastic solutions grossly underestimated hoop stresses for tunnel lining assuming a full-slip condition between linings and the tunnel ground, and that the J.J. Wang (1993) solution was adequate using a no-slip condition. The authors suggest that the Penzien (2000) solution should be avoided (Kontoe et al. 2008).

There are two important points which should be noted regarding the seismic performance of the Bolu tunnels during the 1999 Duzce Earthquake. First, the tunnels were under construction at the time of the earthquake, and second, they were of very close proximity to the fault rupture. Consequently, the unfinished tunnel linings were 
subjected to intense seismic loading in weak fault gouge clay, leading to subsequent failure of linings. The fault gouge clay caved into the tunnel in some sections. This observation contrasts the behavior of mountain tunnels in competent material, where lining collapse rarely leads to total caving of in situ material. Finished tunnel sections on both the Elmalik and Asarsuyu drives performed well during the strong ground shaking, providing confidence that the completed tunnels will have sufficient capacity to withstand significant seismic events. Although not in service or completed at the time of failure, the Bolu twin highway tunnels provide an important case study which can and should be further investigated in order to gain more understanding of the performance of circular tunnels in relatively poor soil conditions.

\subsubsection{Summary of Case Studies}

All of the case studies illustrate differences regarding seismic response of underground structures, depending on factors such as geologic conditions, tunnel geometry, design methods, and construction methods. The Daikai station collapse stresses the importance of designing for transverse racking deformation in shallow cutand-cover tunnels in weak soil. Mountain tunnels act as important lifelines that may are vulnerable to traffic disruption due to lining collapse and tunnel portal blockage. Studies regarding the Bolu twin tunnels suggest that ovaling, ground squeezing, and soil cave-in are major concerns or deep circular tunnels in weak geologic conditions. All of the case studies promote the importance of accounting for USSSI effects for the design of new structures or seismic retrofits of existing structures. 


\subsection{Previous Experimental work}

Research using physical soil-structure interaction testing of underground structures has been limited. Much of the existing experimental literature has been in response to the catastrophic Daikai failure, in which physical testing was used for the development and verification of finite element methods (FEM) for understanding the seismic response of reinforced concrete structures subjected to transverse seismic loading (Matsui et al. 2004; Nam et al. 2006). All of the experimental studies discussed here were performed in 1-g environments. Curiously, centrifuge modeling has not often been employed for USSSI investigations despite its advantages for scale modeling of cohesionless materials.

Although experimental research regarding underground structures is limited, physical testing has been widely and successfully employed for SSI investigation of other types of structures. For example, seismic soil-pile-superstructure interaction (SSPSI) for piles and pile groups has been investigated using full-scale pile loading tests and various forms of model scale testing methods. A comprehensive review of SSPSI testing up to the time of his dissertation is available in Meymand (1998). Additionally, SSI effects on shallow foundations have been investigated using physical testing. Experimental research into investigation of SSI effects on structures other than underground structures is beyond the scope of this thesis. However, the wide range of testing research available contains knowledge of experimental procedures, testing platforms, and analysis methods, which can be replicated and used for scale model testing of different structures. This paper follows the experimental methods and utilizes equipment from Meymand's (1998) study on SSPSI effects in soft clay, akin to that of San Francisco Bay Mud. 


\subsubsection{Experiments on Underground Structures}

Motivated by the failure of the Daikai subway station, Nishiyama et al. (1999) investigated the seismic response of cut-and-cover tunnels through the use of scale model shaking table tests. Model structures were excited with sine waves, and observations were compared with results using various analytical methods. The structures tested represented double and triple cell configurations, and were constructed using aluminum for sidewalls and the floor and ceiling slabs and hard rubber interior walls. The relatively flexible interior walls were meant to simulate the response of flexible interior walls or columns, akin to those that failed in the Daikai station. Silicone was utilized to model soft clay. A primary objective of the study was to examine the magnitude and directivity the shear stresses and normal stresses acting at the soil-structural interface. Thus, the exterior of the model was equipped with two-way load cells and pressure plates.

Experimental results confirmed theoretical shear directions to be accurate along most of the interface, except at the corner of the ceiling slab where shear directivity is reversed. The authors postulate that the normal reaction on the sidewall may have had an influence on shear direction and magnitude, but fail to recognize the possible normal reaction of the silicone on the side of the pressure plate. Results of the testing in conjunction with equivalent linear dynamic analysis (FLUSH) indicated that the directivity of normal forces is a function of the flexibility ratio. The authors suggest that for relatively flexible structures, the ground works to limit deformation through compressive normal forces; and for relatively stiff structures, the ground works to increase deformation through tensile reactions at the soil-structure interface. The latter conclusion does not consider the possibility of gapping or the low tensile capacity of 
soils, especially for cohesionless soils. Experimental racking deformations compared well with analytical methods and dynamic analysis. A related study in Japanese by Muroya et al. (1998) considered the addition of slip layers at the soil-structure interface, satisfying recommendations by Nishiyama et al. (1999).

Che and Iwatate (2002) built a 1/30-scale model cross-section of the Daikai station for shake table testing. The experiment utilized a newly developed laminar container to approximate free-field boundary conditions. Results suggested that the response and dynamic earth pressures on a structure under vertical and horizontal excitation at the resonant frequency are similar to that of horizontal excitation alone. This supports conclusions by field investigations that the Daikai station failed in shear due to horizontal motions. Some important observations of the study are as follows:

- The structure experienced shear and rocking modes due to horizontal excitations and significant strain in center columns.

- Dynamic earth pressures on the ceiling slab were approximately uniform at low excitation levels but not at high levels $(>0.4 \mathrm{~g})$.

- Bending strains are much higher in fixed columns than in columns with flexible joints.

In a later study, Che et al. (2006) recognized the lack of consideration of USSSI for structures that are not critical to life safety. The same laminar box container used for the Daikai station model tests was used for shaking table tests of $1 / 16$-scale model elliptical long span corrugated steel culverts embedded into dry sand. Bending strains on the structure were found to be large under sinusoidal dynamic loading, but not high 
enough to exceed allowable plastic deformation or cause collapse, even at excitation levels close to those recorded during 1995 Kobe earthquake.

Matsui et al. (2004) performed shaking table tests on a double cell rectangular reinforced concrete structures. The aim of the study was to develop and validate a fully dynamic non-linear FEM model, capable of considering bending, racking deformation, reinforcing bar pull out, and soil-structure interface slippage. The reinforced concrete model was fixed to the base of the shake table and embedded in dense cohesionless dry sand. Tests were performed using a laminar box to limit boundary effects. Upon excavation, observations of damage patterns agreed well with measured data. Figure 2.16 shows simultaneous sidewall curvature measurements and dynamic earth pressures measured during excitation. It illustrates that the soil imposes compressive forces resulting in inward curvature of the sidewalls leading to the cracking pattern observed on the inner surface of the sidewall. Cracking at the top and bottom of the sidewall can be attributed to bending, which is consistent with damage observed in the center columns of the Daikai station. Results indicated that the soil distortions controlled the structural distortions. This can be attributed to the low stiffness of the structure compared to the soil as well as hysteretic degradation of structural stiffness. These observations provide insight into the behavior of relatively flexible reinforced concrete structures and respective damage patterns. Experimental results compared well with the dynamic finite element model in terms of racking distortions, damage patterns, shear slippage, and shear stress. Details regarding the applicability of the model to differing soil types or stiffness ratios are not discussed. 

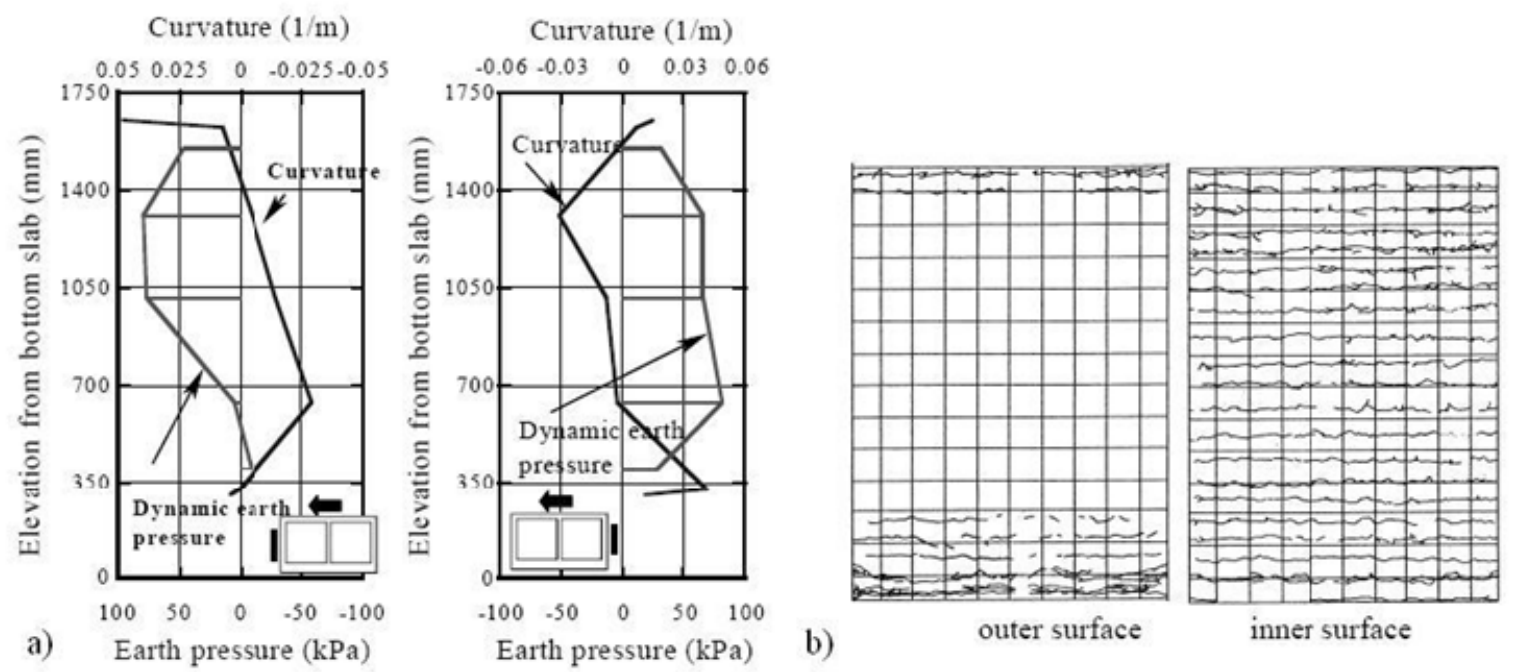

inner surface

Figure 2.16 - Experimental results showing a) the relationship between sidewall curvature and dynamic earth pressures and $b$ ) observed sidewall cracking pattern (from Matsui et al. 2004)

Nam et al. (2006) utilized cyclic loading tests to investigate the seismic response of rectangular reinforced concrete structures embedded in cohesionless soil. A primary focus of the study was to validate a numerical method for considering the interaction at the interfacial zone between the soil and the embedded structure. The interface interaction is modeled as a combination of elastic and plastic response, where deformation of the soil is assumed to occur over a limited thickness at the soil-structure interface. The test setup included uniform vertical pressure distribution to increase overburden, and employs horizontal load distributors mounted on hinges to allow for differential transverse deformations (Figure 2.17a). Experimentally and numerically derived hysteresis curves show reasonable agreement, especially at lower shear distortions. Shown in Figure 2.17c are hysteretic material degradation results from one of two test cases considered in the study (Figure 2.17b). This study illustrates an advantage of cyclic load testing in that it allows for careful and controlled observations of 
material degradation. Unlike in shake table or centrifuge testing, real-time dynamic response is not captured.

a)
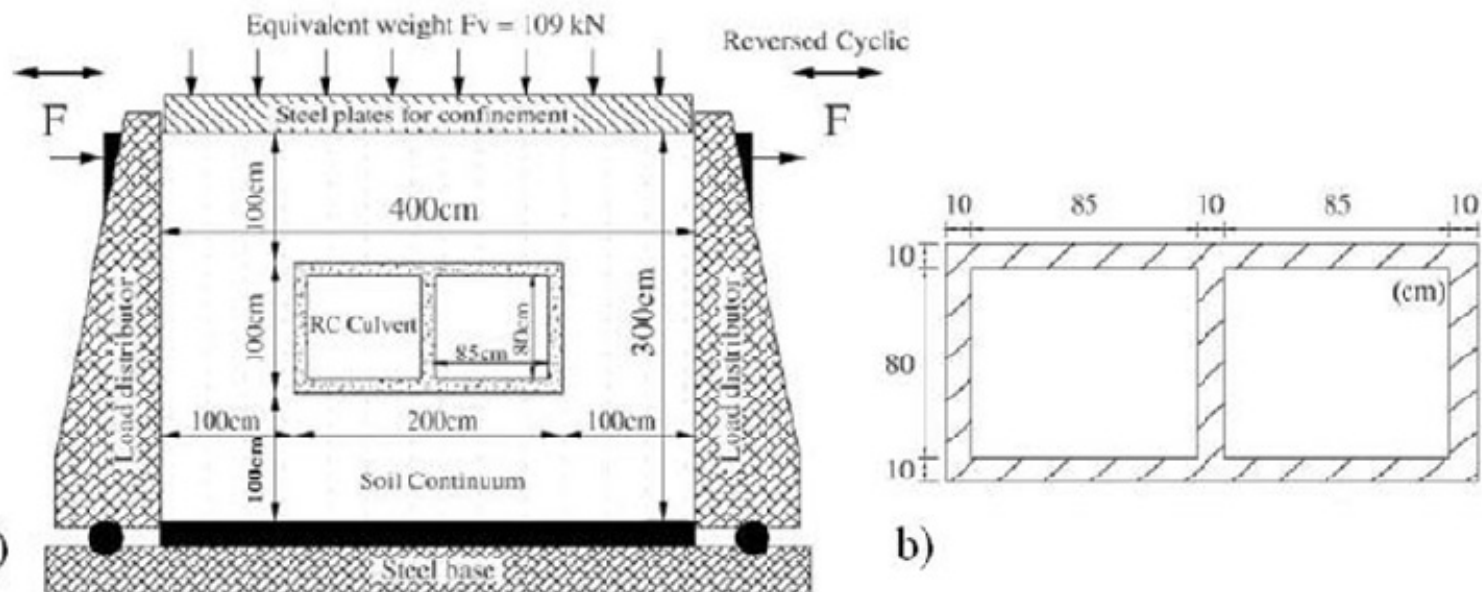

b)

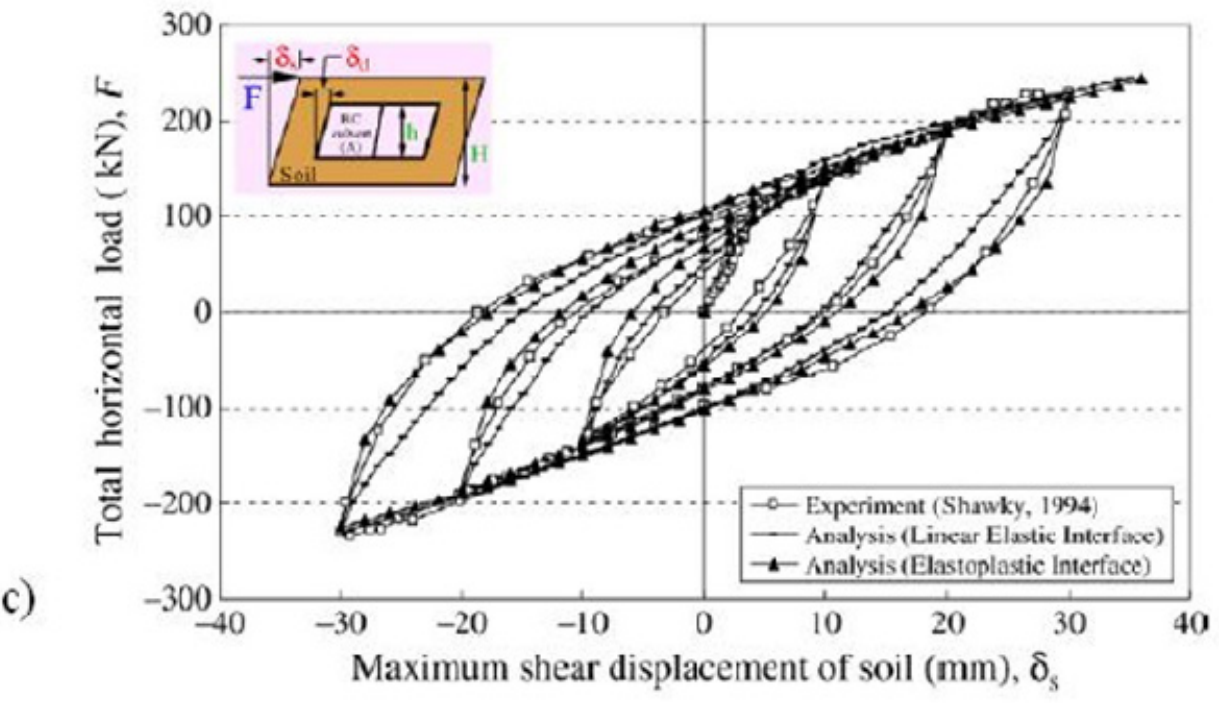

Figure 2.17 - a) cyclic testing platform b) example double-cell cross-section investigated c) experimental hysteretic results compared with analytical results (after Nam et al. 2006) 


\subsubsection{1-g Scale Model Testing of Soft Clay}

Successful modeling of soil in a 1-g environment is a difficult endeavor due to dependency of the behavior of some soils on effective stress. However, the behavior of clay is often defined in terms of undrained strength $\left(S_{u}\right)$, which is relatively independent of confinement provided by overburden pressure. Meymand (1998) developed a platform for scale model shake table testing of piles in clay. The testing platform used flexible waterproof neoprene in conjunction with high strength horizontal Kevlar bands to form a testing container capable of closely modeling free-field conditions of saturated soft clay. Numerical modeling results using QUAD4M illustrate the rationale behind choosing a flexible barrel system by comparing the spectral response of three possible test containers with the response a prototype soil column (Figure 2.18). The study involved developing scale model clay following similitude theory. The clay "recipe" was based on earlier work by Seed and Clough (1963) in which a highly saturated 3:1 mixture of kaolinite to bentonite was used to represent soft clay. The testing platform proved to reasonably model free-field conditions of soft clay in a 1-g scale model environment.

Under loan by the University of California, Berkeley, much of the equipment has been refurbished and modified for use at Cal Poly. As such, the testing platform and methods employed for this research follow that of Meymand's study, and various references will be made to that work. 

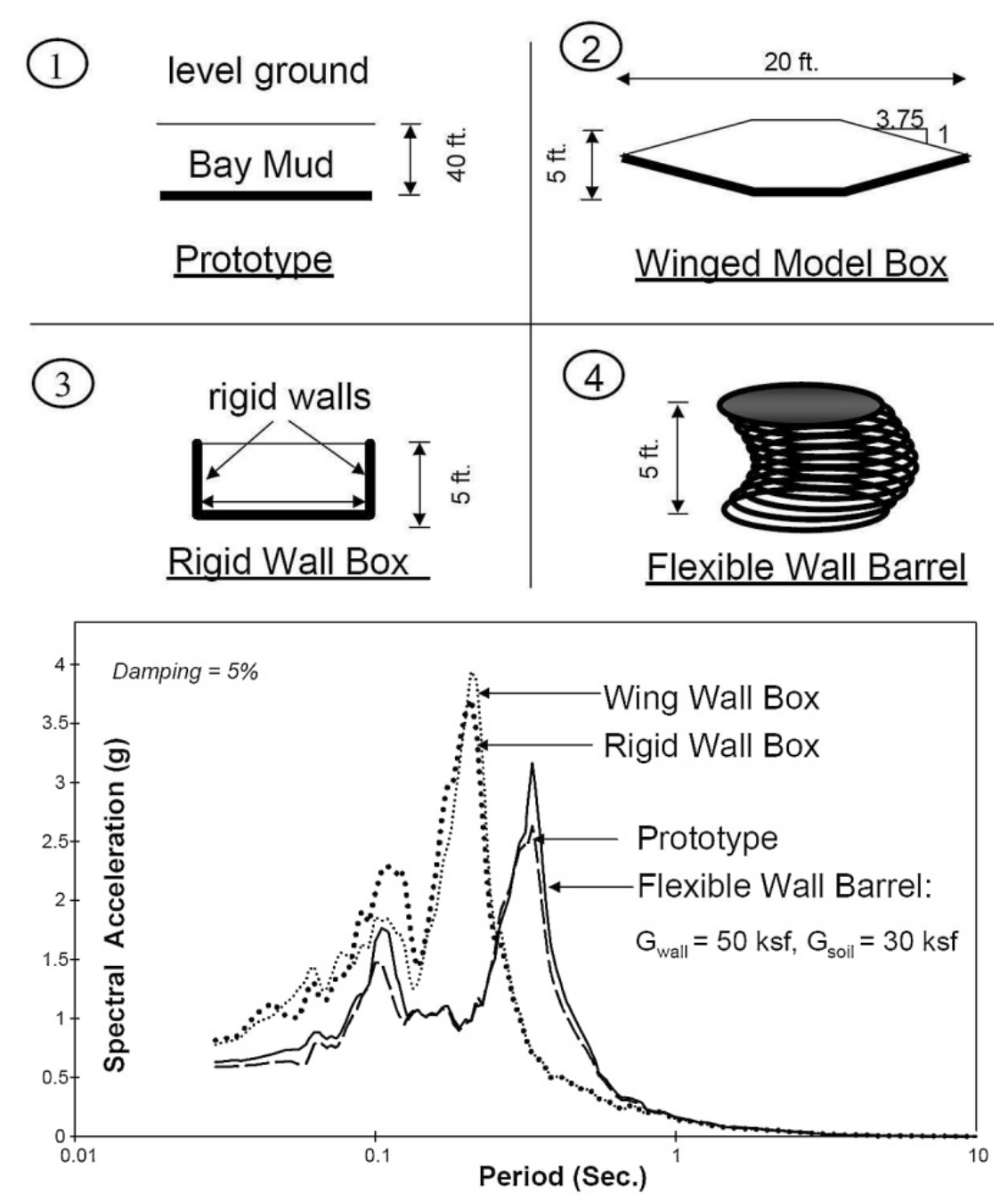

Figure 2.18 - Numerical comparison of the free-field response of model containers with the response of a prototype soil column of soft clay (after Meymand 1998)

\subsubsection{Summary of Previous Experimental Work}

The reviewed literature regarding experimental testing of underground structures provides general overview of USSSI response that compares well with field observations. Damage patterns, deformations modes and stress distributions have been modeled with reasonable accuracy. The studies relating to seismic response of reinforced concrete in particular provide very promising results with regard to modeling complicated non-linear phenomena, especially material degradation and interfacial slippage (Matsui et al. 2004; 
Nam et al. 2006). Nishiyama et al. (1999) reinforced the validity of the well established equivalent linear model FLUSH for evaluating deformation response. Despite these successes, the limited number of experimental investigations regarding tunnel structures parallels the limited field observations discussed earlier and sheds light on some important research needs.

With the exception of Nishiyama et al. (1999), which attempted to model clay using silicone, there is a lack of studies which consider the response of underground structures in clay. Most experimental research has been focused on shallow structures embedded in sand. Moreover, careful similitude analysis does not appear to be considered in any of these experiments. Meymand (1998) argues that similitude is an important consideration in a $1-\mathrm{g}$ environment in order to properly model prototype response. One study attempted to addresses this issue by providing vertical confining pressure for cyclic loading tests (Nam et al. 2006). The studies also lack free-field testing, which is for comparing free-field distortions with structural distortions. Finally, the previous research focused on structures that are less stiff in comparison to the surrounding medium, and experimental research investigating relatively stiff structures is lacking.

Research presented in this thesis attempts to address some of these deficiencies by exploring the USSSI response of a stiff underground structure in soft clay subjected to transverse racking deformation. The study is performed in a 1-g shake table testing environment and utilizes scale model similitude criteria where appropriate. Results are then compared with the equivalent linear numerical results from SHAKE and FLUSH for validation and extrapolation of the test results. 


\section{CHAPTER 3 - DEVELOPMENT OF SCALE MODEL}

\subsection{Introduction}

Physical scale-modeling can be used to study the behavior of complex large scale systems in controlled environments. It is especially useful in situations where large scale testing cannot be readily simulated in the field, of which earthquake investigations are a perfect example. Economic advantages exist as well, due to the reduced size of scale models compared to their full scale counterparts. This study employs scale modeling concepts to develop a shake table testing program for evaluation of seismic performance of underground structures in soft clay. The program consists of scale model shake table testing in a 1-g environment using a flexible walled test container filled with a model soil mix. Testing was conducted in two phases: Phase I consists of free-field testing on the model soil without the inclusion of an embedded structure, and Phase II includes a scale model single cell rectangular subway cross-section embedded in the soil. This chapter begins by describing the development of the scale models and finishes with brief descriptions of a previously developed scale model testing container, adapted for this research.

\subsection{Scale Model Similitude}

Understanding of scaling relations is important for accurately modeling full scale (prototype) behavior at the scale model level. Meymand (1998) explains that

development of proper scaling relations is desired because scale models are often used to make predictions of prototype response or provide calibration benchmarks for analytical 
methods. Scale model similitude describes the relationship between model and prototype behavior, and it is the basis for determining scaling relations.

Moncarz and Krawinkler (1981) present varying levels of scale model proficiency. "True" models require that similitude relationships are satisfied for all parameters in a system. Such models are difficult to achieve due to the complex nature of engineering problems. "Adequate" models correctly scale primary factors, but compromise for secondary variables in a manner that minimizes errors in model prediction. "Distorted" models will not accurately predict prototype response, unless difficult compensations are applied to prediction equations or physical quantities. Considering this, the proficiency of a similitude model is highly dependent on its method of derivation.

Various methods for deriving scaling relations have been developed for scalemodeling applications. Meymand (1998) describes three methods (from simple to complex) in a comprehensive review of similitude in geotechnical applications: dimensional analysis, similitude theory, and the method of governing equations. Dimensional analysis aims to convert a dimensionally homogeneous equation to an equation consisting of dimensionless factors based on the fundamental "measures-ofnature" (mass, length, and time). Similitude theory, also known as the Buckingham Pi Theorem, takes dimensional analysis a step further by identifying and accounting for the unique forces acting in a system for the formation of dimensionless terms. The method of governing equations is the most complex and powerful of the three methods. It requires the transformation of a system described by a complex differential equation to a non-dimensional form. 


\subsubsection{Development of Similitude Criteria}

Dimensional analysis is used as the basis for deriving scale relations in this study due to the desire to the parallel test conditions and testing platform under Meymand's SSPSI study and. Specific engineering parameters are reduced to the fundamental MassLength-Time $(M-L-T)$ units and scaling relations are derived using corresponding scale factors for mass $(\mu)$, length $(\lambda)$, and time $(\tau)$. These relations are all then evaluated in terms of the geometric scaling factor $(\lambda)$. In this manner, scaling relations can be defined for all of the pertinent variables identified in the study. For dimensional analysis in this testing program, the following three quantities map 1:1 between scale and prototype:

1. soil density $\left(\rho_{s}\right)$, because similar density to the prototype is desired

2. acceleration $(A)$, because testing is performed in 1-g environment

3. strain $(\varepsilon)$, because strain is a dimensionless quantity Applying these conditions, scaling factors can be systematically derived in terms of the geometric scaling factor $(\lambda)$, where scale factors are the ratio of prototype (subscript $p$ ) to model (subscript $m$ ). The following examples show the process of deriving scaling factors for mass $(\mu)$, force $(F)$, time $(\tau)$, and shear wave velocity $\left(V_{S}\right)$.

Definitions for scale factors for mass $(\mu)$, length $(\lambda)$, and time $(\tau)$ :

$$
\mu=\frac{M_{p}}{M_{m}} \quad \lambda=\frac{L_{p}}{L_{m}} \quad \tau=\frac{T_{p}}{T_{m}}
$$

Mass:

$$
\frac{\left(\rho_{s}\right)_{p}}{\left(\rho_{s}\right)_{m}}=1=\frac{\left(M_{p} / L_{p}^{3}\right)}{\left(M_{m} / L_{m}^{3}\right)}=\left(\frac{M_{p}}{M_{m}}\right)\left(\frac{L_{m}^{3}}{L_{p}^{3}}\right)=\frac{\mu}{\lambda^{3}}
$$




$$
1=\frac{\mu}{\lambda^{3}} \quad \Rightarrow \quad \mu=\lambda^{3}
$$

Force:

$$
\frac{F_{p}}{F_{m}}=\left(\frac{M_{p}}{M_{m}}\right)\left(\frac{A_{p}}{A_{m}}\right)=\left[\frac{\left(\rho_{s}\right)_{p}}{\left(\rho_{s}\right)_{m}}\right]\left(\frac{L_{p}^{3}}{L_{m}^{3}}\right)\left(\frac{A_{p}}{A_{m}}\right)=(1) \lambda^{3}(1)=\lambda^{3}
$$

Time:

$$
\begin{aligned}
& \frac{A_{p}}{A_{m}}=1=\frac{\left(L_{p} / T_{p}^{2}\right)}{\left(L_{m} / T_{m}^{2}\right)}=\left(\frac{L_{p}}{L_{m}}\right)\left(\frac{T_{m}^{2}}{T_{p}^{2}}\right)=\lambda\left(\frac{T_{m}^{2}}{T_{p}^{2}}\right) \\
& \left(\frac{T_{p}^{2}}{T_{m}^{2}}\right)=\tau^{2}=\lambda \quad \Rightarrow \quad \tau=\sqrt{\lambda}
\end{aligned}
$$

Shear Wave Velocity:

$$
\frac{\left(V_{s}\right)_{\mathrm{p}}}{\left(V_{s}\right)_{\mathrm{m}}}=\frac{\left(L_{p} / T_{p}\right)}{\left(L_{m} / T_{m}\right)}=\left(\frac{L_{p}}{L_{m}}\right)\left(\frac{T_{m}}{T_{p}}\right)=\left(\frac{\lambda}{\sqrt{\lambda}}\right)=\sqrt{\lambda}
$$

Any desired scaling factor can now be derived in terms of factors $\mu, \lambda$, and $\tau$ as shown in the following derivation of the soil shear modulus $\left(G_{S}\right)$ scale factor (units of stress):

$$
\frac{\left(G_{S}\right)_{\mathrm{p}}}{\left(G_{S}\right)_{\mathrm{m}}}=\left[\left(\frac{M A}{L^{2}}\right)_{p} /\left(\frac{M A}{L^{2}}\right)_{m}\right]=\frac{\mu \frac{\lambda}{\tau^{2}}}{\lambda^{2}}=\frac{\mu}{\lambda \tau^{2}}=\frac{\lambda^{3}}{\lambda(\sqrt{\lambda})^{2}}=\lambda
$$

Iai (1989) explains that earlier similitude investigations into dynamic response of soil employed the Buckingham Pi Theorem, resulting in relations only applicable to soil 
shear deformation modes. Therefore, he recognized the need to develop generalized scaling relations that could be applied to a soil-structure-fluid system under dynamic loading. Iai's complex set of scaling relations are derived from geometric, density, and strain scaling factors. Meymand observed that scaling relations developed using dimensional analysis agree with Iai's set when the shear wave velocity scaling factor is equal to the square root of the geometric scaling factor $(\sqrt{\lambda})$, This is because Iai's strain scaling factor $\left(\lambda_{\varepsilon}\right)$ is:

$$
\lambda_{\varepsilon}=\frac{\lambda}{\left[\left(V_{s}\right)_{\mathrm{p}} /\left(V_{s}\right)_{\mathrm{m}}\right]^{2}}
$$

Equation 3.7 in Equation 3.9 gives:

$$
\lambda_{\varepsilon}=\frac{\lambda}{(\sqrt{\lambda})^{2}}=\frac{\lambda}{\lambda}=1
$$

This result satisfies the condition that strain scales 1:1 under dimensional analysis. Also, the shear wave velocity scaling factor partially satisfies a condition for 1-g scale modeling presented by Moncarz and Krawinkler (1981) called the Cauchy condition. The Cauchy condition states that, for material modulus $(E)$ and density $(\rho)$, the following should be satisfied to constitute a "true" model (Moncarz and Krawinkler 1981):

$$
\frac{\left(V_{s}\right)_{\mathrm{p}}}{\left(V_{s}\right)_{\mathrm{m}}}=\sqrt{\frac{\left(E_{p} / \rho_{p}\right)}{\left(E_{m} / \rho_{m}\right)}}=\sqrt{\lambda}
$$


Meymand (1998) calls this a "necessary requirement for simultaneous replication of restoring forces, inertial forces, and gravitational forces in a dynamic model system." This condition severely limits material selection because it requires the model material to have small modulus and high mass density, or both. Obtaining such materials may not feasible. Thus, adjustments may be needed to achieve an "adequate" scale model. One way is to apply "distributed" or "lumped" masses to the model in a manner that is seismically effective without significantly affecting the structural configuration. Another approach is to ignore gravitational effects, which in some cases can be appropriate (Moncarz and Krawinkler 1981).

As previous field investigation, testing, and analyses have shown, USSSI effects are highly dependent on kinematic interaction and mostly independent of inertial interaction. For this reason, the density of the structural system is not considered a primary variable for similitude, so the condition expressed in equation 3.11 is relaxed. Also, the soil consists of saturated clay, and for undrained conditions, the stress-strain behavior is not dependent on confinement due to overburden pressure (Meymand 1998). Under these conditions, gravitational force effects can be ignored in the similitude model without resulting in a distorted model. This greatly simplifies the development of scaling relations, by permitting the use of Iai's scaling relations for all pertinent quantities.

\subsubsection{Summary of Similitude Criteria}

Primary factors for scaling relations can be identified by carefully considering the components of an USSSI system. These components include the site response, kinematic interaction, interfacial shear, and damping. Construction issues cannot be easily 
predicted in a prototype situation and for this reason are not integrated into the similitude model. Important parameters for USSSI effects are shown in Table 3-1.

Table 3-1: USSSI components and associated variables (adapted from Meymand, 1998)

\begin{tabular}{|l|l|}
\hline \multicolumn{1}{|c|}{ USSSI Interaction Mode } & \multicolumn{1}{c|}{ Variables } \\
\hline 1 Free-field Site Response & $\begin{array}{l}\text { shear wave velocity }\left(V_{s}\right) \\
\text { soil density }\left(\rho_{s}\right) \\
\text { modulus degradation }\left(G / G_{\text {max }}\right) \text { and } \\
\text { damping }(\beta)\end{array}$ \\
\hline 2. Transverse Kinematic Interaction & $\begin{array}{l}\text { Free-field site response and... } \\
\text { flexural rigidity }(E I) \\
\text { structural geometry }(L)\end{array}$ \\
\hline 3. Interfacial Shear Interaction & $\begin{array}{l}\text { interface contact }(\text { full confinement }) \\
\text { soil shear strength }\left(S_{u}\right) \\
\text { interface adhesion }(\alpha)\end{array}$ \\
\hline 4. Damping & $\begin{array}{l}\text { Free-field site response and... } \\
\text { material modulus }(E) \\
\text { structural mass }(M) \\
\text { structural geometry }(L)\end{array}$ \\
\hline
\end{tabular}

Examining Table 3-1, material mass $(M)$ poses a challenge for proper scale modeling, but correct scaling of mass is mostly important for radiation damping. Meymand (1998) postulates that at high shaking levels, characteristic of those planned for this testing program, radiation damping should have little influence. Interface adhesion $(\alpha)$ is not directly quantified in the design of the model, but this is a secondary concern in soft clay conditions. Proper modeling of adhesion is more critical in cohesionless soil applications.

Considering the discussion presented here with regard to similitude criteria and the components of USSSI, Table 3-2 presents a list of selected scaling relations for this testing program. Various scale factors may apply to multiple pertinent engineering 
quantities, as long as the dimensions are the same. All factors are presented in terms of the geometric scaling factor $(\lambda)$ and are calculated for $\lambda=10$, which was selected for this research.

Table 3-2: Scale factors of selected engineering variables in terms of the geometric scaling factor $(\lambda)$ (adapted from Iai, 1989; Meymand, 1998)

\begin{tabular}{|l|c|c|}
\hline Variable & Scale Factor & For $\lambda=10$ \\
\hline Soil Density & 1 & 1 \\
\hline Force* & $\lambda^{3}$ & 1000 \\
\hline Stiffness* & $\lambda^{2}$ & 100 \\
\hline Modulus & $\lambda$ & 10 \\
\hline Acceleration & 1 & 1 \\
\hline Shear wave Velocity & $\lambda^{1 / 2}$ & 3.16 \\
\hline Soil Damping & 1 & 1 \\
\hline Poisson's ratio & 1 & 1 \\
\hline Time & $\lambda^{1 / 2}$ & 3.16 \\
\hline Frequency & $\lambda^{-1 / 2}$ & 0.316 \\
\hline Length & $\lambda$ & 10 \\
\hline Stress & $\lambda$ & 10 \\
\hline Strain & 1 & 1 \\
\hline Flexural Rigidity* & $\lambda^{5}$ & 100000 \\
\hline Dimensionless Quantities & 1 & 1 \\
\hline
\end{tabular}

*These quantities may be conveniently expressed per longitudinal length (breadth) of a tunnel by dividing the scale factor by the geometric scale factor

\subsection{Development of Scale Model Soil}

Model soil used in this research is nearly identical to that of Meymand's (1998) study investigating SSPSI effects. Extensive research and development went into the model soil in order to conform to the complex scale modeling criteria. The model soil was designed to conform to scaling laws for both free-field conditions and soil-pile interaction conditions. Strain in the stiff underground model is expected to be relatively 
small. This simplifies the model soil criteria in that large inelastic deformation effects such as gapping are not expected to be pronounced.

Meymand identified five discrete, non-linear soil parameters affecting model soil response: density, modulus reduction and damping, stress-strain response, shear wave velocity, and undrained shear strength. The soil density scales approximately 1 to 1 between prototype and model due to the limited range of soil density possible in nature. Modulus degradation and damping are not modeled based on a selected prototype, but are considered under the method of implied prototypes. This is an iterative procedure in which an acceptable range of prototype behavior is identified, and models are built and tested to verify similarity. The process is best described in the flowchart in Figure 3.1. Scale modeling criteria is complicated by the competing scale factors for shear wave velocity $(\sqrt{\lambda})$ and undrained shear strength $(\lambda)$. These criteria must be reasonably satisfied to sufficiently model small-strain elastic response and large strain inelastic response, controlled by shear wave velocity and undrained shear strength respectively. Ultimately, the San Francisco Bay Mud, was chosen as the implied prototype for development of the soil model (Meymand 1998). 
Identify primary modes of system response and variables contributing to these modes

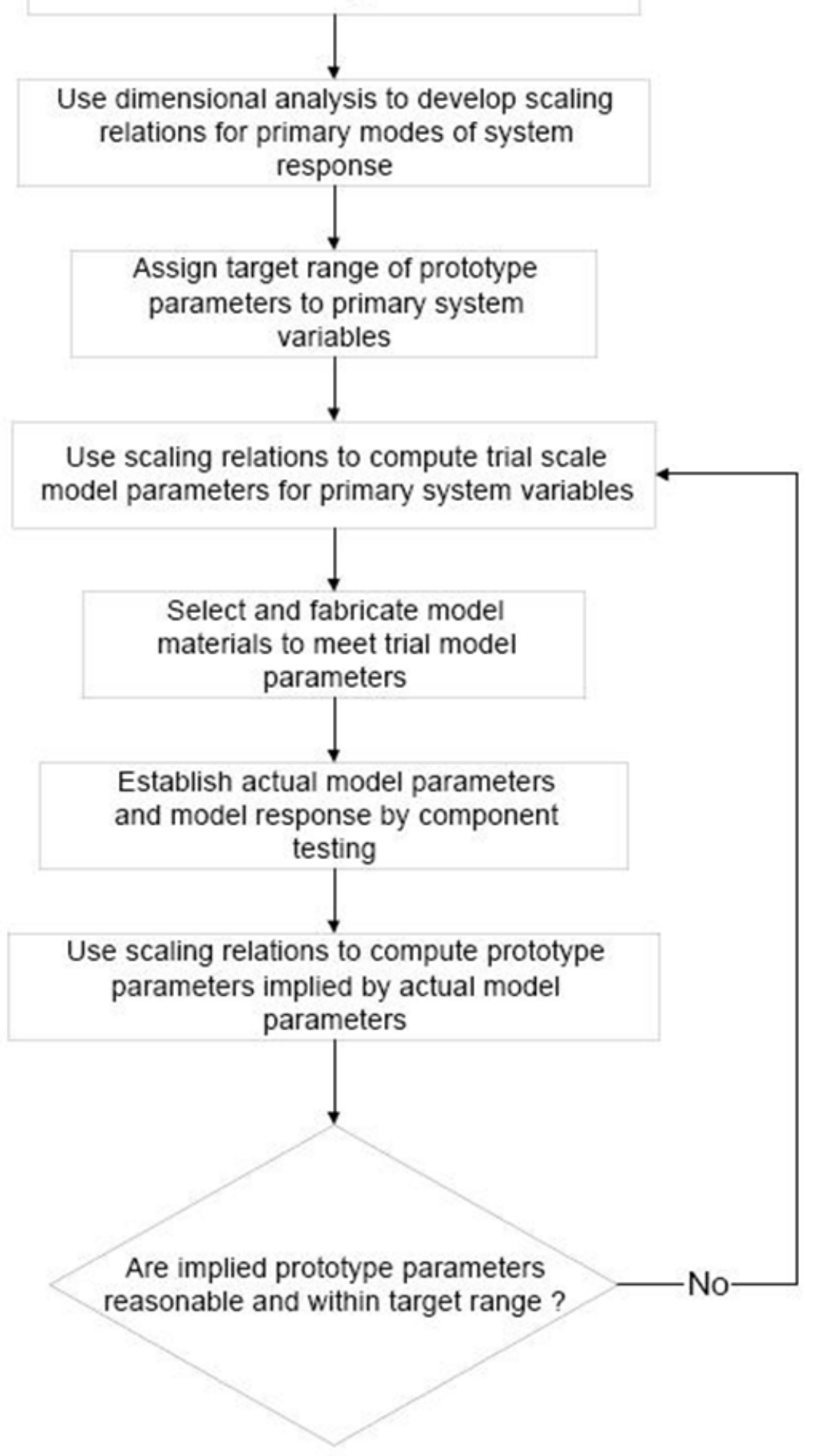

Figure 3.1 - Flowchart describing the method of implied prototypes, used to develop the model soil (from Meymand 1998) 


\subsubsection{Model Soil Design and Testing}

The model soil mix design was adapted from a "recipe" that has been used extensively at UC Berkeley to model soft clay. The kaolinite/bentonite mix was originally used for scale model shaking table investigations into the seismic response of earth dams in which dynamic shear strength was the primary variable of interest (Seed and Clough 1963). Similar mixes were later used for various studies involving fault rupture and seismic slope stability. Considering the importance of shear wave velocity on small strain free-field behavior, Meymand (1998) sought to improve the recipe by using admixtures to increase small strain dynamic stiffness (i.e. shear wave velocity) without significantly increasing undrained shear strength. After testing various admixtures, class C fly ash was identified to have the desired effects. Results of lab testing performed by various researchers are compiled and discussed in detail in the SSPSI study and are briefly described here.

- Meymand (1998) used unconsolidated undrained triaxial testing (UUTX) to investigate the effects of fly ash on undrained shear strength. Test results supported the conclusion that varying water content significantly affects undrained shear strength, while fly ash content has little influence. Also, a consolidation test was performed on a model specimen with $100 \%$ water content and $10 \%$ class $\mathrm{C}$ fly ash, resulting in a coefficient of consolidation of $6.5 \times 10^{-3}$

$\mathrm{m}^{2} /$ year. Meymand suggests that "this slow rate of consolidation implied relatively stable soil properties throughout the shaking table testing time window." 
- Gruber (1996) performed unconsolidated undrained (UU) and UUTX tests on model soil containing $10 \%$ class C fly ash and Bay Mud to investigate and compare the stress-strain behavior of model and prototype soils under static and dynamic loading. Results indicate that, unlike the more sensitive prototype, the model soil acts as a strain hardening material under confined conditions. The yield strain was similar for both model and prototype soil. Dynamic strength increase was higher for the prototype soil compared to the model soil, approximately $75 \%$ and $25 \%$ respectively. Meymand (1998) concluded that although the model soil did not perfectly replicate the stress-strain behavior of Bay Mud, it provides a reasonable response under the method of implied prototypes. A static strength reduction factor of 0.75 for model soil static undrained shear strength was adopted to reflect these results.

- Wartman (1996) used bender element testing to investigate the effect of fly ash as an admixture in the model soil. It was concluded that class $\mathrm{F}$ fly ash simply acted as an inert filler material, while class $\mathrm{C}$ fly ash caused an appreciable increase in small strain stiffness without significantly influencing undrained shear strength. This helps to satisfy the competing scale factor criteria for shear wave velocity and undrained shear strength.

Based on these test results, an original model clay design was selected consisting of $67.5 \%$ kaolinite, $22.5 \%$ bentonite, and $10 \%$ class C fly ash with $100 \%$ water content. A similar mix design was chosen in this study, with the exception of an increase in water 
content, which will be discussed in detail in following sections. Shown in Table 3-3 is a comparison between design model soil properties and typical Bay Mud properties.

Table 3-3 Selected properties for prototype and model soil mix in SSPSI study (after Meymand 1998)

\begin{tabular}{|l|l|l|}
\hline Property & Bay Mud & Model Soil \\
\hline Saturated Unit Weight $\left(\mathrm{kN} / \mathrm{m}^{3}\right)$ & 14.8 & 14.8 \\
\hline Water Content $(\%)$ & 90.00 & 100.00 \\
\hline Liquid Limit (\%) & 88.00 & 115.00 \\
\hline Plastic Limit (\%) & 48.00 & 40.00 \\
\hline Plasticity Index (\%) & 40.00 & 75.00 \\
\hline Coefficient of Consolidation Cv $\left(\mathrm{m}^{2} /\right.$ year) & 0.75 to 0.92 & $6.5 \times 10^{-3}$ \\
\hline Undrained Shear Strength $(\mathrm{kPa})$ & 29 to 57 & 4.1 \\
\hline Shear wave Velocity $(\mathrm{m} / \mathrm{s})$ & 114 to 160 & 40.0 \\
\hline
\end{tabular}

Values for undrained shear strength for the Bay Mud are derived from a relationship with shear wave velocity developed by Dickenson (1994), who investigated seismic site response in the San Francisco Bay Area after the 1989 Loma Prieta Earthquake. This relationship is shown in Figure 3.2 and can be described by equation 3.12, where $V_{s}$ is in feet per second (fps) and $S_{u}$ is in pounds per square foot (psf).

$$
V_{s}=18\left(S_{u}\right)^{0.475}
$$

For this study, UU triaxial testing on mixes near $110 \%$ water content showed approximate undrained shear strengths ranging between $3 \mathrm{kPa}(60 \mathrm{psf})$ and $5 \mathrm{kPa}(100$ psf). Issues regarding soil sampling and load cell precision were encountered increasing the uncertainty; therefore, these values should be considered estimations. Nonetheless, they do provide a qualitative range of expected $S_{u}$ which agrees well with the more 
reliable lab testing results reported in Meymand (1998). Converting this strength range to prototype static Su strength values using $\lambda=10$ and the strength reduction factor 0.75 gives $22 \mathrm{kPa}(450 \mathrm{psf})$ to $37 \mathrm{kPa}(750 \mathrm{psf})$. The Dickenson relationship reveals prototype shear wave velocity range of $100 \mathrm{~m} / \mathrm{s}(330 \mathrm{ft} / \mathrm{s})$ to $130 \mathrm{~m} / \mathrm{s}(420 \mathrm{ft} / \mathrm{s})$, corresponding to model shear wave velocity of $32 \mathrm{~m} / \mathrm{s}(105 \mathrm{ft} / \mathrm{s})$ to $40 \mathrm{~m} / \mathrm{s}(130 \mathrm{ft} / \mathrm{s})$ using the shear wave velocity scale factor $(\sqrt{\lambda})$

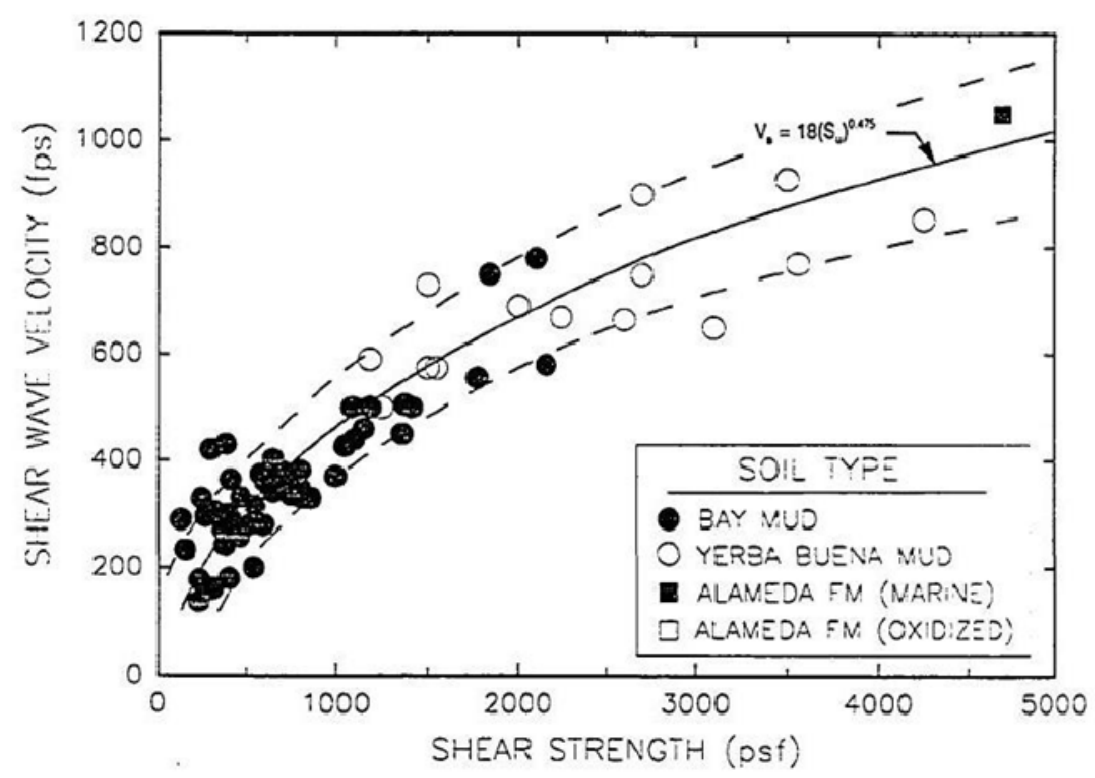

Figure 3.2 - Relationship between shear wave velocity and static undrained shear strength of shallow cohesive soils (from Dickenson 1994)

\subsubsection{Final Model Soil Design}

This study employs essentially the same mix design as Meymand's study, with the exception that the model materials were selected based on availability. However, care was taken to find materials as close to the original as possible. The bentonite is American Standard 200 mesh, distributed by Scott Sales Company in Huntington Park, CA; the Kaolinite is Kamin 35 (formerly Huber 35), distributed by the PT Hutchins 
Company in City of Industry, CA; and the class C fly ash was obtained through Mineral Resources Technologies, a subsidiary of Cemex USA.

Target water content had to be increased after trial and error during full scale mixing. It was found that the mixing equipment cannot sustain operation at water content of $100 \%$, especially due to dramatic stiffening effects provided by the fly ash. The target water content in this research was chosen as $125 \%$, which is near the operational limit of the mixing equipment. More details on the mixing equipment and its operation are provided in Chapter 4 and Appendix A.

\subsection{Development of Scale Model Tunnel}

The model tunnel section used in this shake table study is based on typical tunnel cross-sections found in cut-and-cover sections of the Bay Area Rapid Transit (BART) rail system. Two prototype cross-sections are considered in the design of the model scale tunnel. The first is a double cell section, which is adapted into a single cell section by simply considering one half of the structure (Figure 3.3). The second is a typical single cell section with stiffening haunches in the upper corners (Figure 3.4). This cross-section was used by Ostadan and Penzien (2001) for a numerical investigation of USSSI effects for a BART extension to the San Francisco Airport. Figure 3.4 provides a range of section dimensions, which promotes the method of implied prototypes for the model section design. Therefore, constructing an exact replica of a prototype section was not a priority in the scale model section design, as it would only complicate the scale modeling effort and introduce further uncertainty in scale model system. 


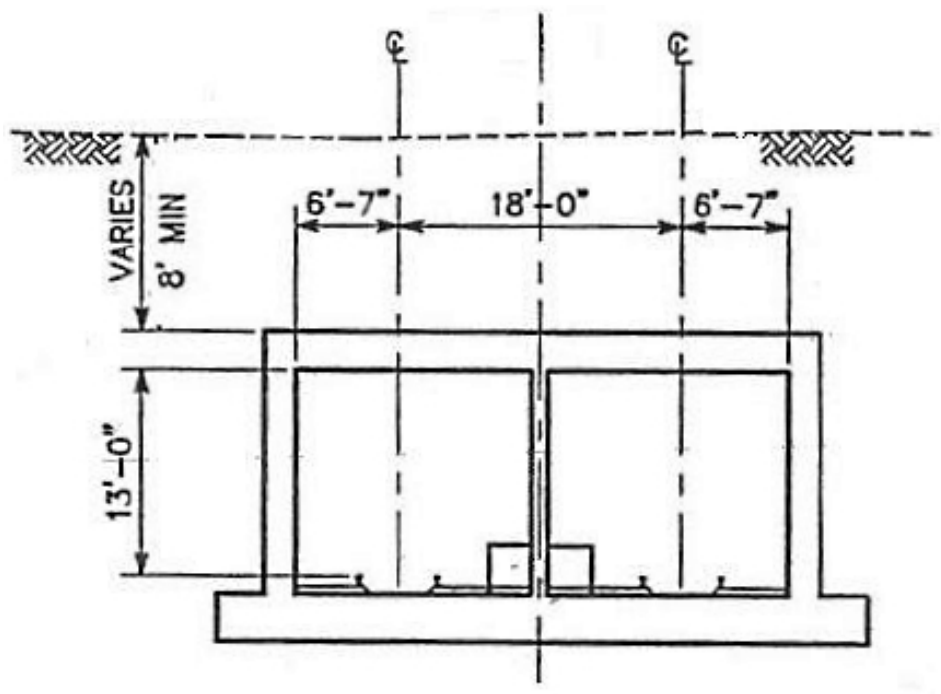

Figure 3.3 - Typical double cell cut-and-cover subway cross-section (from BART)

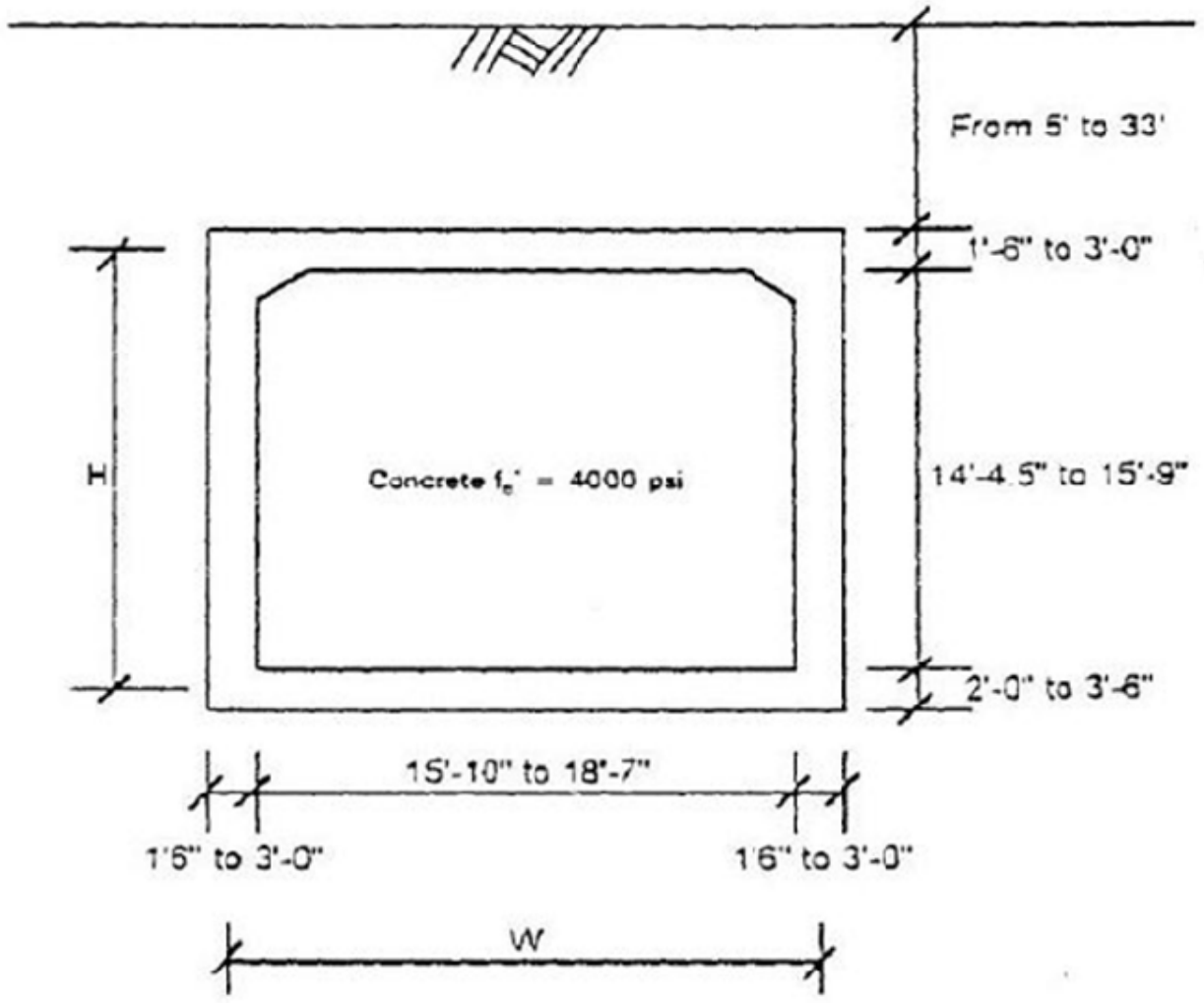

Figure 3.4 - Typical single cell cut-and-cover BART cross-section with upper corner haunches (from Ostadan and Penzien 2001) 


\subsubsection{Selection of Geometric Scale Factor}

This study employs a geometric scale factor of 10 based on the physical constraints of the testing container, the typical prototype cut-and-cover sections, and the desire not to significantly vary from the scaling factor of 8 used for Meymand's (1998) pile study. A simple pseudo-static analysis was performed considering the mass of a soil wedge extending from the edge of the test container to the sidewall of a subway crosssection. The sidewall dimensions were taken as median values from the single cell section in Figure 3.4. Reaction force on the sidewall was considered to be $75 \%$ of the weight of the soil wedge. This reflects a horizontal acceleration of $0.75 \mathrm{~g}$, similar to shaking intensity expected during testing. The influence of the tunnel sidewall reaction on the edge of the test container was evaluated using elastic solutions by Westergaard and Bousinesq. A scale factor of 10 was shown to limit sidewall reaction influence on the container wall to $5 \%$ (Figure 3.5). Based on the conservative nature of the analysis, this scale factor is considered sufficient to limit boundary effects.

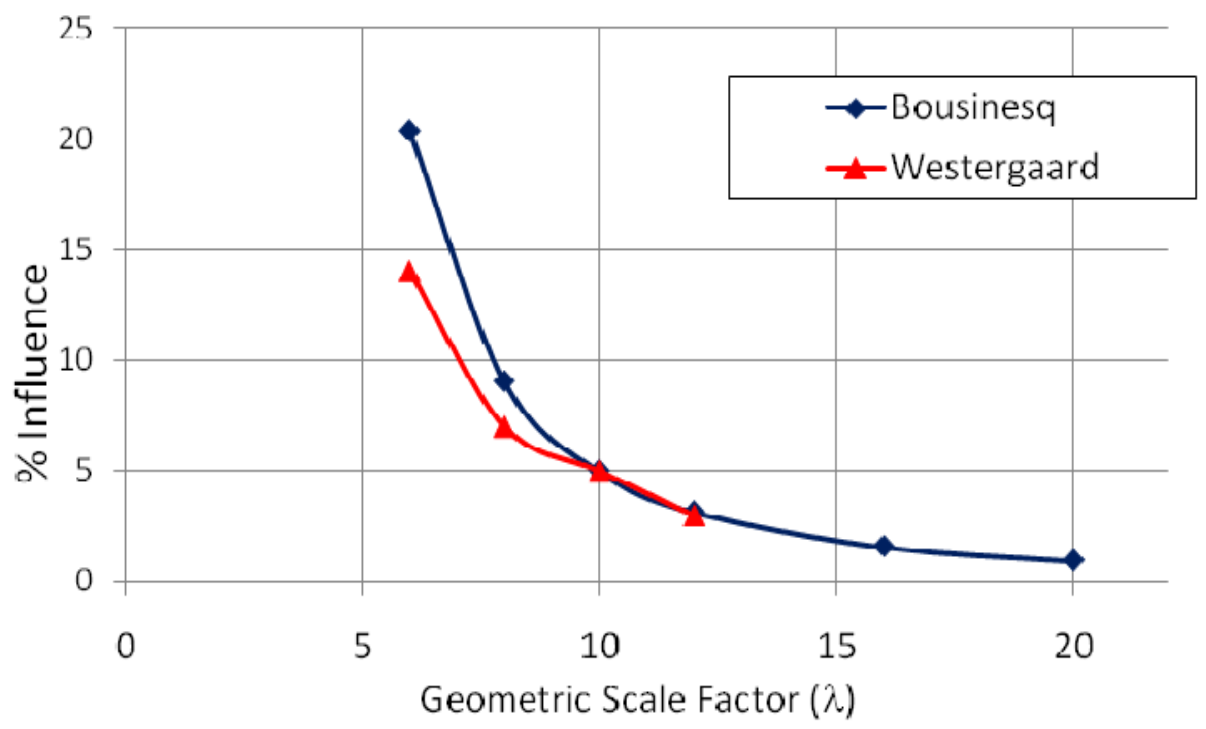

Figure 3.5 - Pseudo-static analysis results of influence of soil wedge reaction on the structural sidewall at the edge of the test container 


\subsubsection{Scale Modeling Criteria for Tunnel Section}

Structural stiffness was identified as the most important contributing variable affecting USSSI response and therefore constitutes the bulk of the scale modeling effort. The kinematic interaction mode in the USSSI system is simulated by carefully scaling the relationship between structural stiffness and soil stiffness. Contributing factors that affect structural stiffness include structural geometry, flexural rigidity (EI), and ductility.

Geometry of the structure was determined based on the geometric scale factor and the prototype cross-sections. A preliminary geometric design was derived from combining the outside dimensions of one cell of the double cell structure (Figure 3.3) with approximately the median wall-thickness of the single cell structure (Figure 3.4). The result is subsequently scaled by the geometric scale factor of 10 . For simplicity in structural calculations and model constructability, the thickness of the side walls, ceiling slab, and floor slab were taken to be equal.

The flexural rigidity is a function of wall/slab thickness, material modulus, and loading conditions. The loading conditions in this study are modeled as a simple shear racking model, where axial rigidity of the structural members is not considered. For determining wall thickness, one of two options may be applied. The first option is to strictly adhere to geometric scaling criteria by fixing the wall thickness to reflect the prototype exactly. The second option is to allow wall thickness to vary, thereby basing material selection primarily on flexural modulus. Option two was chosen for this study in order to open up greater material selection possibilities. This philosophy was selected under the assumption that wall thickness alone plays a minor role in seismic response compared to overall effect of flexural rigidity and structural stiffness. 
Ductility is most important when exploring modes of failure in a structural system, such as studies that explored the failure of the Daikai subway station. This study examines the response of a very stiff section with low ductility in soft clay. Based on the small deformations expected in such a system, an uncracked reinforced concrete section was selected as the prototype. In this way, complex non-linear phenomena such as concrete degradation, steel yielding, and reinforcement pull-out can be ignored. The structure is modeled as a very stiff elastic system, which may be similar to some structures conservatively designed using the free-field deformation approach. Modeling of an elastic system also simplifies material selection and construction.

Examining Table 3-1, kinematic interaction is accounted for in the free-field soil response in combination with the flexural rigidity and structural stiffness. Interface shear interaction is dependent on the soil-structure contact and the interface friction, which is not formally characterized. However, the highly cohesive nature of the soft model clay limits the possibility of significant interface slip making it a relatively minor concern; therefore it is not directly accounted in the scale modeling effort.

Material damping is not considered at the model scale, since it would require building a scale model reinforced concrete structure, which still may not result in properly scaled material damping characteristics. Hysteretic damping of the soil is accounted for in the model soil design and is acceptable based on the method of implied prototypes (Meymand 1998). Proper scaling of radiation damping effects requires modeling of the high frequency vibration characteristics of the structure, which is dependent on the mass of the material. The vibration characteristics of the structure are 
not accounted for in the scale model as correct scaling of material mass would greatly constrain material selection possibilities.

\subsubsection{Material Selection}

Material selection for the model was based on economy, ease of fabrication and constructability, material availability, material modulus, chemical reactivity, and water resistance. Other than slab thickness, geometry of the structure was not considered in the selection of materials, since it is essentially fixed based on prototype geometry. It was desirable to use a water resistant material for the protection of instrumentation and to maximize the life of the model. This requirement essentially limits material selection to metals and plastics. Another concern was stability of model material under chemical attack from the highly caustic class $\mathrm{C}$ fly ash.

Recognizing that the flexural rigidity under horizontal racking deformation is only a function of slab thickness and material modulus, a spreadsheet solution was developed to explore variation of the two factors. In order to further ease the material selection, prototype concrete strength was also allowed to vary between $31.0 \mathrm{MPa}$ (4500 psi) to 41.4 MPa (6000 psi). This is based on the common condition of actual concrete strength being greater than design strength, often $27.6 \mathrm{MPa}$ (4000 psi). Selection preference within this range was biased toward the upper end to reflect the limited ductility and higher member stresses of stronger concrete. Since the section is considered uncracked, modulus was based solely on concrete strength using the equation:

$$
E_{c}=57000 \sqrt{f_{c}^{\prime}}
$$


where $E_{c}$ is the flexural modulus of concrete and $f^{\prime}{ }_{c}$ is the concrete strength. This is an empirical equation where both values are in units of pounds per square inch (psi).

Based on the selected concrete strength range, the modeling of an uncracked section, and the scale factor for material modulus $(\lambda)$, the target range for model modulus of elasticity was indentified to be $2.62 \mathrm{GPa}(380 \mathrm{ksi})$ to $3.03 \mathrm{GPa}$ (440 ksi). This is much lower than most metals, which generally have a flexural modulus greater than $70 \mathrm{GPa}$ $(10,000 \mathrm{ksi})$. Selection of any metal as the model material would result very thin wall thickness, which is problematic due to the high sensitivity of flexural rigidity to variations in thickness as well as the susceptibility of some metals to corrosion.

Plastics provide a suitable model material because there is a wide range of material properties available, chemical reactivity is low, and fabrication is straightforward. The two materials that best suit the criteria are polycarbonate (common trade name Lexan) and acrylic (common trade name Plexiglas). Of the two, acrylic is the less expensive option and is significantly easier to fabricate. The mean modulus of elasticity of acrylic is $3.31 \mathrm{GPa}(480 \mathrm{ksi})$, which is close to the selected target range. The maximum thickness of commercially available cast acrylic is $50.8 \mathrm{~mm}$ ( 2 inches), and was chosen as the model thickness. The resulting flexural rigidity of the scale model is approximately $36 \mathrm{kNm}^{2}$ (per longitudinal meter of the tunnel). Keeping in mind the range of concrete strength previously discussed, this model flexural rigidity implies the following example prototype structural configurations:

- $\quad 31.0 \mathrm{MPa}$ concrete with wall and slab thickness of $55 \mathrm{~cm}(\lambda=10.8)$

- 41.4 MPa concrete with wall and slab thickness of $52 \mathrm{~cm}(\lambda=10.3)$ 


\subsubsection{Final Structural Configuration}

The final structural design consists of a single cell rectangular cross section with a height of $55 \mathrm{~cm}$ and a width of $58 \mathrm{~cm}$. The original acrylic sheet dimensions limited the length of the structure to $61.4 \mathrm{~cm}$. Subject to slight spatial variations in manufacturing tolerance, the nominal thickness for structural sidewalls and floor and ceiling slabs is $50.8 \mathrm{~mm}$. Considering Figure 2.3, the flexibility ratio can be calculated from the simple solution for a one barrel frame structure with equal moment of inertia $(I)$ for the wall, floor and ceiling as (J. J. Wang 1993):

$$
F=\frac{G_{m} L}{S_{1} H}=\frac{G_{m}}{24}\left(\frac{H^{2} L+H L^{2}}{E I}\right)
$$

Where: $H=$ height of structure; $L=$ width of structure; $E I=$ flexural rigidity (per longitudinal unit of tunnel); $G_{m}=$ soil shear modulus, and $S_{1}=$ unit racking stiffness (per longitudinal unit of tunnel). For a unit concentrated force, the expression for $S_{1}$ can be derived from equation 3.14 as:

$$
S_{1}=\frac{1}{\Delta}=\frac{24 E I}{H^{3}+H^{2} L}
$$

The model design is for a cross-section of tunnel independent of length, so all design parameters are described per longitudinal length of the tunnel (meter). The actual length of the model is constrained by the geometric limitations of the test container and material. Transverse racking behavior should not be affected by the length of the model, unless it is long enough to be subject to $3 \mathrm{D}$ effects. Figure 3.6 shows the final structural configuration of the model section. 

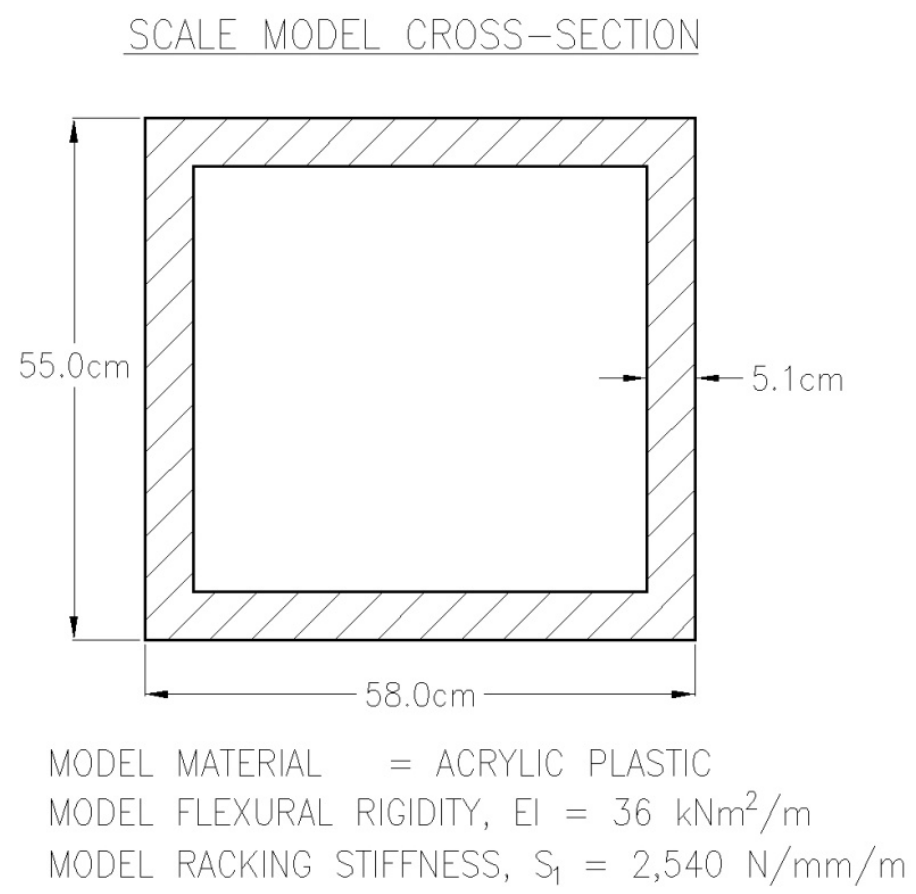

Figure 3.6 - Model scale subway cross section design and associated engineering parameters

\subsection{Selection of Input Motions}

The ground motions used in this test program were selected specifically to impose large adverse on an underground structure, and are the following:

1. 1979 Imperial Valley, El Centro motion (Figure 3.7)

2. 1992 Landers, Joshua Tree motion (Figure 3.8)

3. 1999 Chi Chi, TCU075 motion (Figure 3.9)

These were also the same motions selected and peer reviewed for a BART tunnel consulting project where the subway section was similar to the prototype being investigated in this study. Both horizontal azimuths from each motion were run through the shake table. To adhere to the similitude analysis and provide the correct dynamic response, time is scaled at $\lambda^{0.5}$. This means the time step of the ground motions are compressed to $\Delta \mathrm{t} / \lambda^{0.5}$. For this study $\lambda=10$, so the time step of the motions are 
compressed by a factor of 3.16. These motions are also corrected for full ground reflection, because they were recorded at the ground surface but are used as the shake table input at the base of the flexible wall barrel. This was accomplished by using the deconvolution capabilities in SHAKE91 (Idriss and Sun 1992). The full reflection of an "outcrop" motion was subtracted to render a "within" motion with respect to the prototype soil profile. The scaled input motions are shown in the following figures.
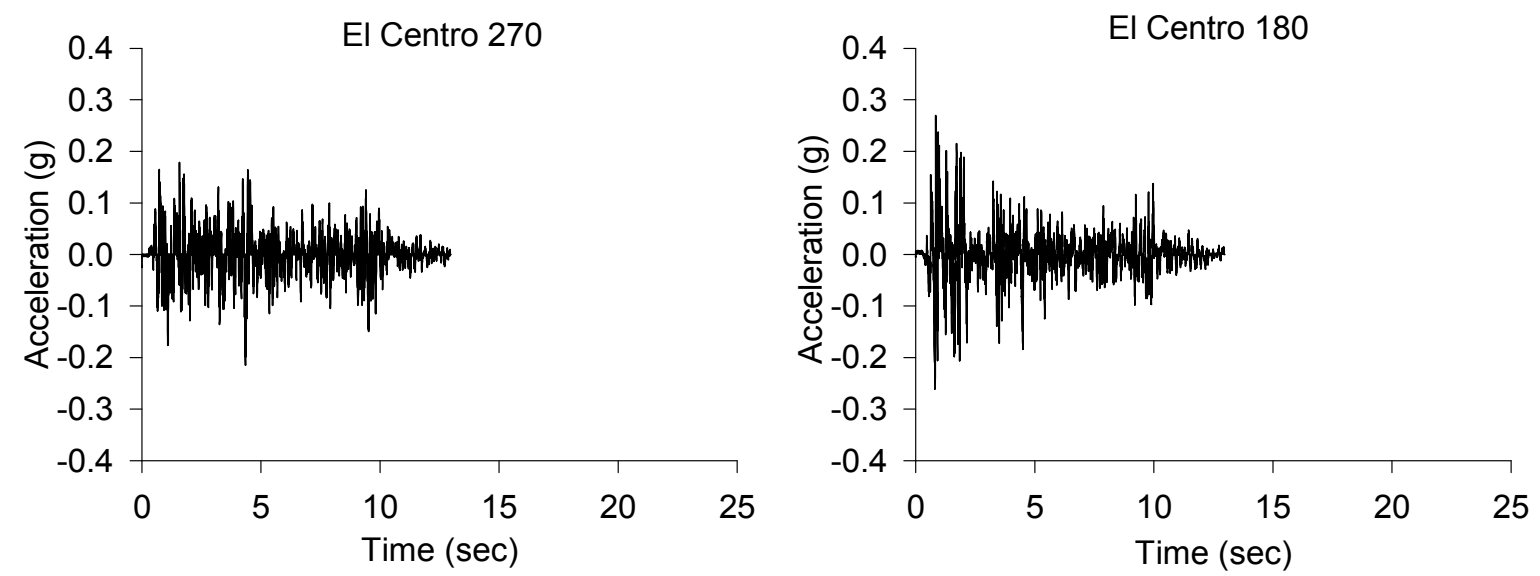

Figure 3.7 - 1979 Imperial Valley earthquake time histories, both horizontal azimuths of the El Centro motion corrected for full reflection with the time step scaled by $\lambda^{0.5}$
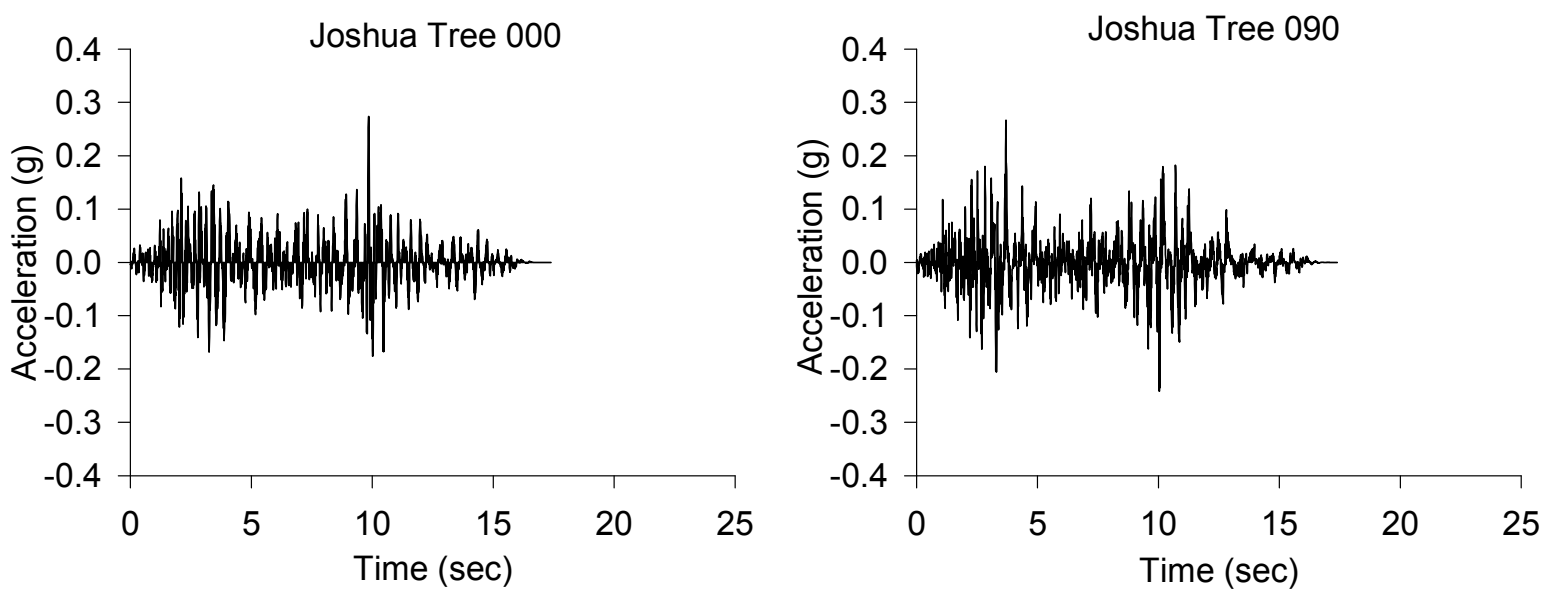

Figure 3.8 - 1992 Landers earthquake time histories, both horizontal azimuths of the Joshua Tree motion corrected for full reflection with the time step scaled by $\lambda^{0.5}$ 

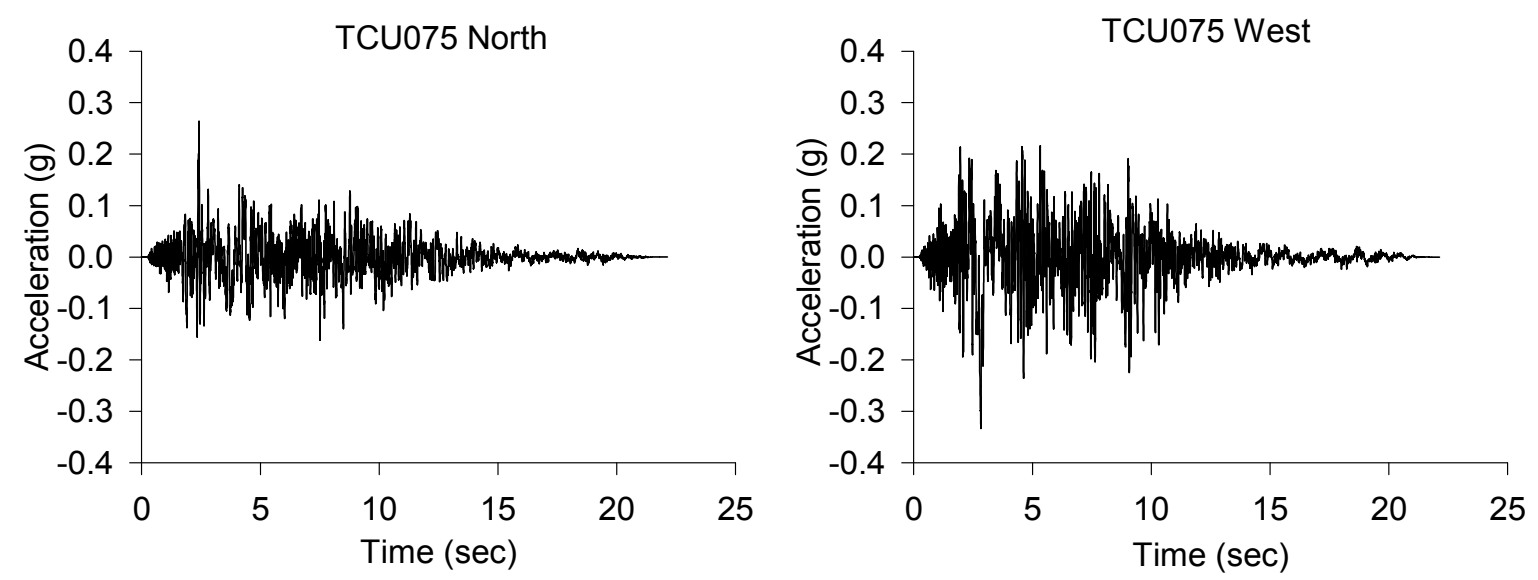

Figure 3.9 - 1999 Chi Chi earthquake time histories, both horizontal azimuths of the TCU075 motion corrected for full reflection with the time step scaled by $\lambda^{0.5}$

\subsection{Scale Model Testing Container}

A flexible wall test container is used in this study for the purpose of confining a circular column of soil while still allowing for free translational movement in all horizontal directions. The flexible wall significantly limits the rigidity of the soil boundary allowing for a close approximation of free-field conditions when subjected to primarily horizontal motion. Meymand (1998) originally designed and developed the container at University of California, Berkeley for seismic SSI research into piles. The container and associated equipment is on loan for use in this study. Over a decade of storage resulted in degradation of certain important components. Therefore, refurbishment and replacement of degraded components was deemed necessary. Also, some primary structural components required modification to fit the Cal Poly shake table. Aside from a limited amount of custom fabrication, the specifications for replacement parts closely follow Meymand's work. Shown in Figure 3.10 is the fully assembled test container standing 1.5 meters in height and capable of confining a saturated soil column with a diameter of 2.3 meters. 


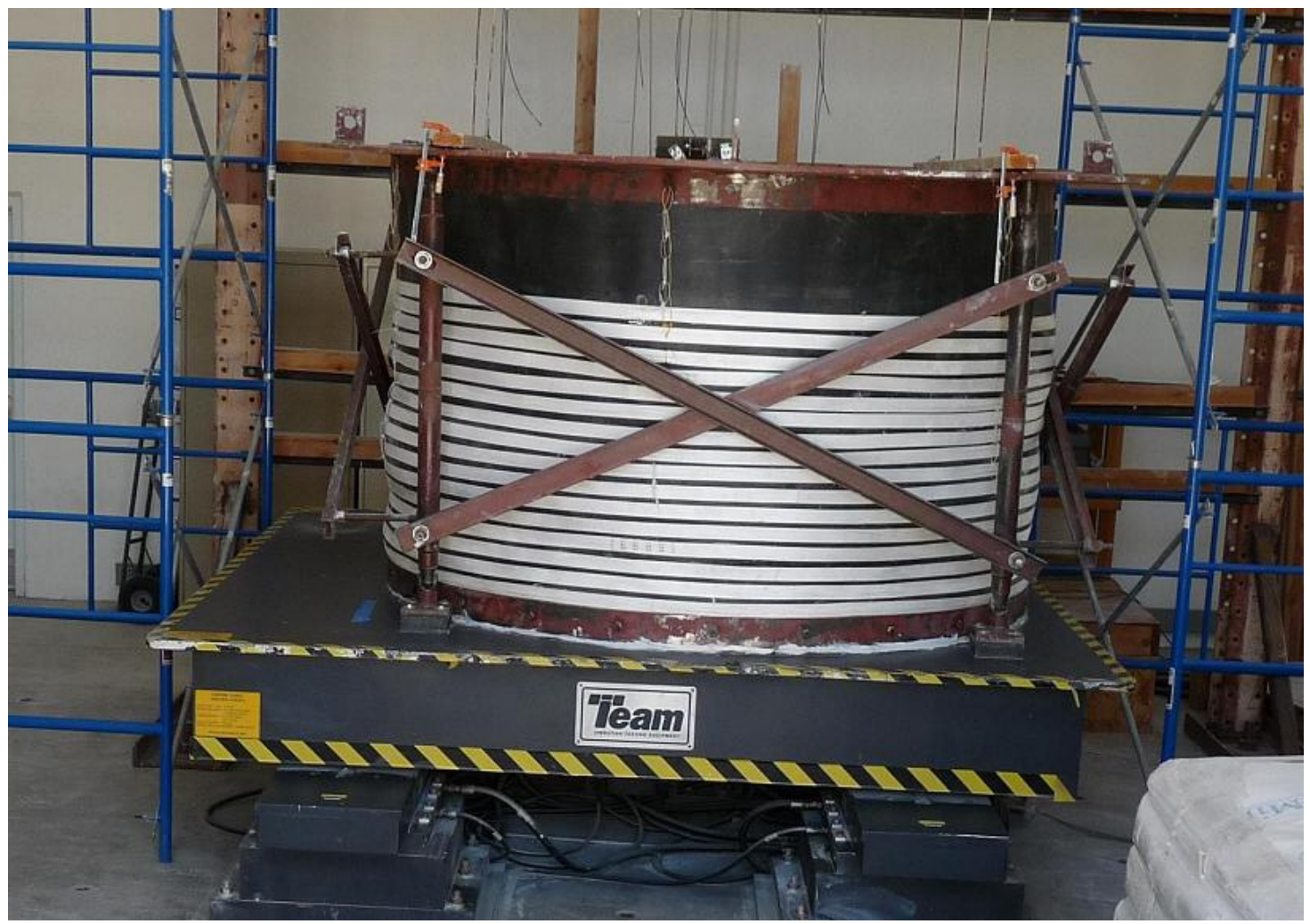

Figure 3.10 - Fully assembled testing container mounted to the shake table (the crossbraces are removed during testing)

A steel skeleton structure was developed to hold the cylindrical shape of the test container. Four heavy steel columns support a steel ring at the top of the container, and a circular steel base plate provides the floor of the soil column. The top ring and base plate are fabricated from $16 \mathrm{~mm}$ thick steel. The columns consist of heavy duty steel tubes with an outside diameter of $73 \mathrm{~mm}$ welded to universal joints near the ends. This provides full horizontal, translational, and rotational freedom of the soil column (Meymand, 1998). Full 3D freedom, as provided by the universal joints, is a remnant of the requirements of the pile study and is not required for 1D shaking table testing.

The universal joints necessitate the use of steel cross braces to keep the container upright during assembly and model construction, and are removed during testing. The 
channel section braces are fitted diagonally connecting adjacent columns using threaded rods welded to the columns. Base adapters for the columns were fabricated from $51 \mathrm{~mm}$ thick by $150 \mathrm{~mm}$ by $150 \mathrm{~mm}$ steel blocks for compatibility with the shake table.

Additional fabrication work on the columns was required to shorten the container from 2.4 meters to 1.5 meters, thereby reducing the test soil column depth by the same amount. This was done in order to meet the lower weight capacity of the Cal Poly shake table. Sections of the columns between the universal joints were cut out and discarded, and the remaining sections welded back together to form the shorter columns. An epoxy and gravel mix was applied to the top surface of the base plate to improve friction between the soil and plate during testing.

Primary soil column confinement is provided by a rubber membrane bolted between the top ring and the circular base using two piece compression rings. The membrane is composed of a single $6.4 \mathrm{~mm}$ thick neoprene sheet fabricated as an open ended cylinder with a single vertical seam. The hardness of the neoprene is 40 durometer on the Shore A hardness scale. Western Rubber and Supply Inc. supplied the membrane and fabrication services. Exterior pieces of the compression rings consist of $5 \mathrm{~mm}$ thick, $102 \mathrm{~mm}$ wide steel bands welded around the inner circumference of the top ring and the outer edge of the base plate. Complementary semicircle bands, two each for the top ring and base plate, make up the interior components of the compression rings.

Further confinement is provided by a series of $45 \mathrm{~mm}$ wide woven fiber bands spaced on center every $60 \mathrm{~mm}$ around the circumference of the membrane, which are designed to carry the hoop stresses and limit excessive bulging of the rubber. The bands are made from high strength Spectra with a minimum breaking strength of 11,000 lbs. 
The original test container utilized Kevlar bands, but the mechanical properties of the two materials are similar. Used in combination, the neoprene membrane and Spectra bands allow the wall to sufficiently confine the soil column, while maintaining radial stiffness and full lateral flexibility (Meymand, 1998). Additionally, the neoprene provides a sufficiently watertight container for testing of saturated soils, especially when used in combination with $100 \%$ silicone caulking to seal possible leak points. Twelve $150 \mathrm{~mm}$ wide textured geomembrane strips (40 mil GSE HyperFrictionFlex) are hung vertically from the top ring to the base plate along the inner circumference of the container. Meymand explains that the "strips provide a path for complementary shear stresses developed in the soil to be carried in the container."

Confidence in the testing container is backed by the extensive research and development performed at UC Berkeley. Prior to construction of the original flexible wall, a suite of tests and analyses were performed in order to provide insight on strength and material requirements for the container design. This included analytical procedures to evaluate the hoop stresses and wall pressures in the model container and triaxial pressure tests on radially confined rubber membranes. From this work, a target material modulus for the membrane and required spacing of bands were determined to limit bulging of the model container during shaking (Meymand 1998). The replacement neoprene membrane and Spectra bands are very similar to the original materials, so they are considered suitable for the research herein. Closely matching the original material specifications is conservative since the soil column in the modified container is shorter, resulting in lower stresses on the components under similar shaking intensity. 


\section{CHAPTER 4 - MODEL CONSTRUCTION AND TESTING}

\subsection{Introduction}

This chapter provides details of testing equipment, instrumentation, setup, and procedures. Most of the procedures described here are a result of extensive trial and error, and should be considered subject to improvement for future projects. In particular, there is significant room for improvement in mixing procedures of the model soil, placement of instrumentation within the soil, and characterization of shear wave velocity profiles. Detailed test setup information is provided for both phases of testing: Phase I being free-field validation of the test container and Phase II being a USSSI investigation of a shallow stiff rectangular structure embedded in soft clay.

\subsection{Shake Table Testing Facility}

The Parson's Earthquake and Geotechnical Engineering Lab houses a majority of the equipment used in this study including the shake table, hydraulic control system, shake table controls, data acquisition module, and overhead crane. The shake table was manufactured by Team Corporation and has a 3 meters square testing surface. It can produce one dimensional (1D) horizontal motion with acceleration up to $10 \mathrm{~g}$ 's and velocity up to $97.5 \mathrm{~cm} / \mathrm{s}$. The total dynamic table stroke is $26.7 \mathrm{~cm}$ and the operation frequency range is 0.1 to $50 \mathrm{~Hz}$. The actuators can produce dynamic force up to $169 \mathrm{kN}$ under a maximum payload capacity of $9000 \mathrm{~kg}$. Figure 4.1 is a view of the shake table facility showing the control area in the foreground and the shake table, testing container, overhead crane, data acquisition module, and other equipment in the background. 


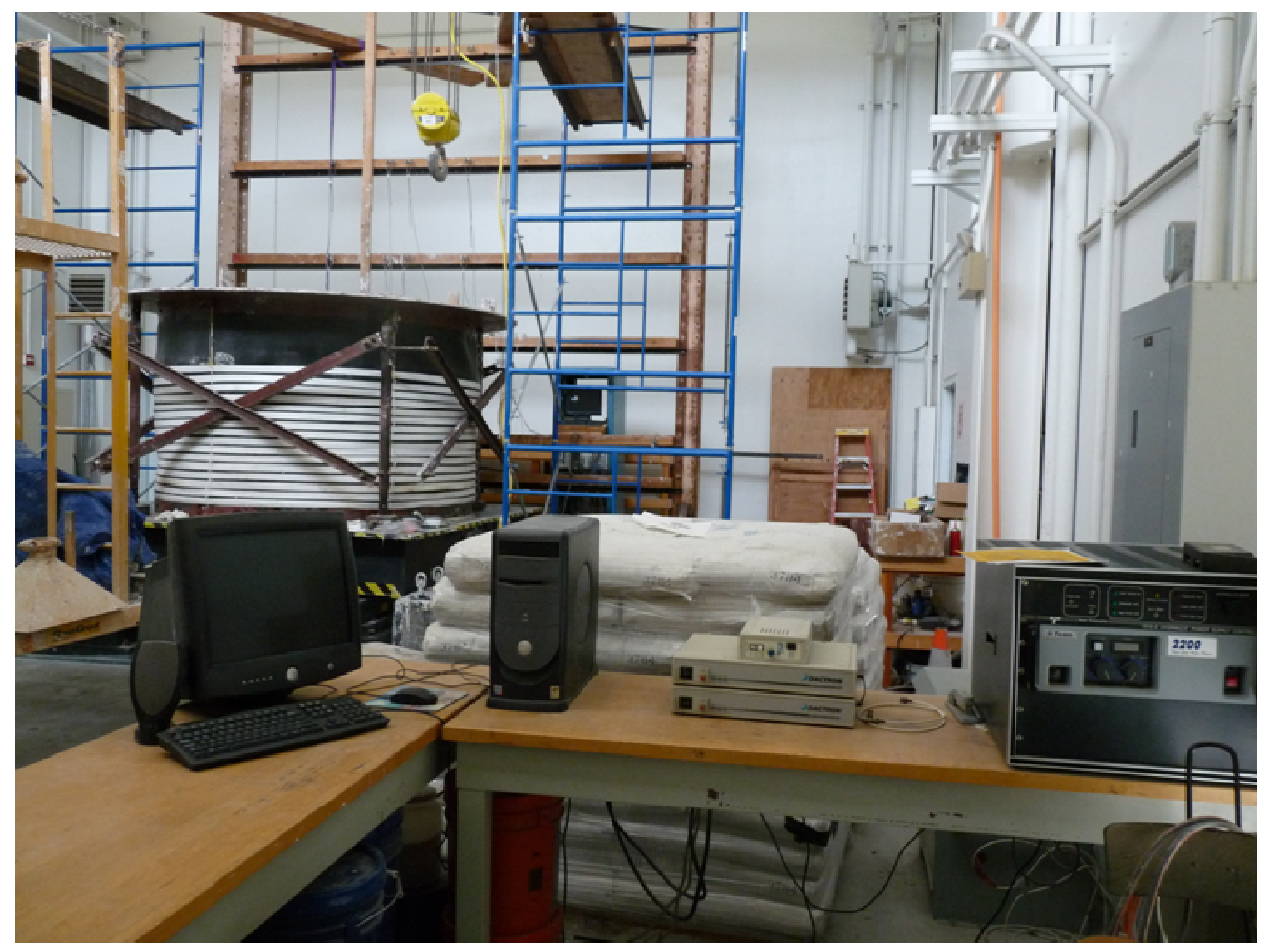

Figure 4.1 - Interior overview of Parson's Earthquake and Geotechnical Engineering Laboratory

Shake table actuators are hydraulically powered by a 60 hp hydraulic power supply (HPS) unit. The Team HPS 2200 valve driver works as the control feedback system to keep the actuator in the desired position and couples with the Dactron shaker control units to drive the shake table during testing. Table acceleration feedback to the shaker control units is handled by a PCB Piezotronics model 482A22 signal conditioner and a single PCB model 353B52 ICP accelerometer mounted to the shake table. This accelerometer also provides the table acceleration output record. The shake table control console is a PC loaded with Dactron Shaker Control Laser software. Input of a desired 
motion is permitted provided the motion does not exceed the dynamic table limitations. A test will automatically abort if the motion does exceed table limitations.

The signal conditioning and data acquisition module consists of a PC equipped with a National Instruments SCXI 1001 chassis that accommodates up to twelve SCXI signal conditioning units. Installed on the PC is National Instruments NI-DAQ data acquisition software and LabView, which provides a convenient block diagram interface for configuring data output files and real time data monitoring.

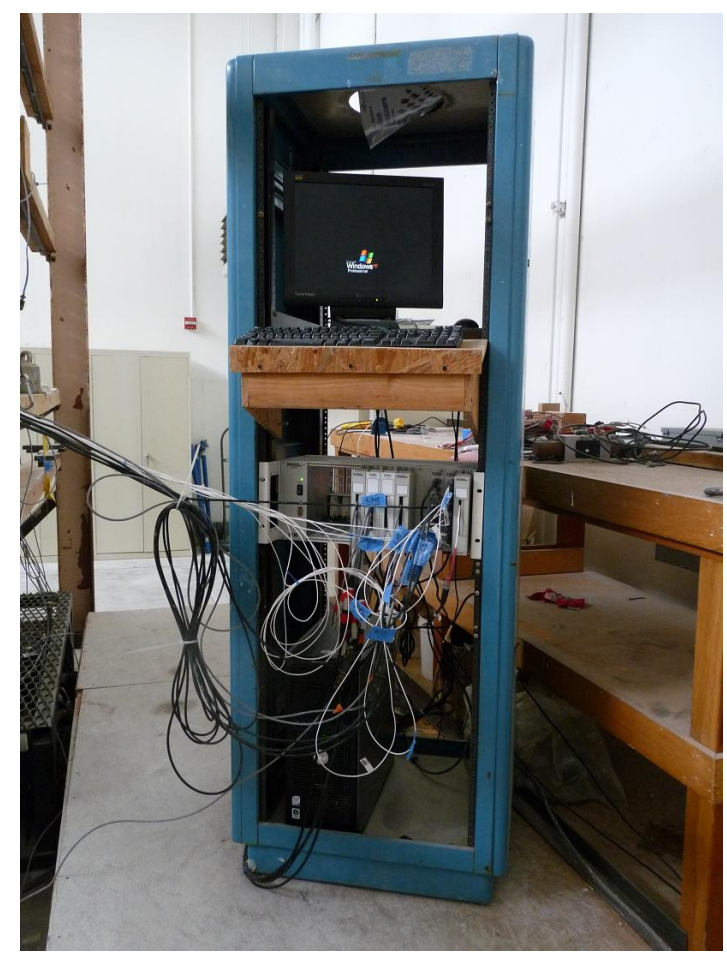

Figure 4.2 - Signal conditioning and data acquisition module with instrumentation wiring

\subsection{Instrumentation}

Multiple types of data were collected during shake table testing. Phase I testing employed horizontal and vertical accelerometer arrays to record acceleration within the soil column for both seismic shake table testing and characterization of shear wave 
velocity profiles. Wire displacement potentiometers (or "wire pots") were utilized for measuring the absolute displacements of the soil column. T-bars fitted with a load cell were used for estimating soil strength. Phase II testing utilized similar instrumentation with the addition of linear variable differential transformers (LVDT) for measuring the internal racking distortions of the model subway section, and accelerometers within the subway section for measuring structural accelerations.

\subsubsection{Accelerometers}

Selection criteria for accelerometers in the model soil include the following: cost, high sensitivity, frequency response range, small size, moisture resistance, and compatibility with existing equipment at Cal Poly. Model 393B04 seismic ICP accelerometers, manufactured by PCB Piezotronics, were selected to measure the accelerations within the soil (Figure 4.3a). These supplemented the existing collection of model J353B51 ICP accelerometers by the same manufacturer, which were used to measure structural accelerations within the subway (Figure 4.3b). A single model 353B52 ICP accelerometer is mounted to the shake table to record table acceleration data and to provide table control feedback during testing (Figure 4.3c).

ICP or Integrated Circuit Piezoelectric is PCB's trade name for accelerometers that contain internal signal conditioning, generically known as Integrated Electronics Piezoelectric (IEPE). Integrated circuitry allows for internal conversion of the high impedance voltage signal generated by the piezoelectric sensing element to a low impedance signal that can be readily transmitted with minimal signal degradation through a coaxial cable. The piezoelectric sensing element (usually quartz or ceramic) responds 
to a mass with an applied acceleration by generating a proportional electrical output.

This voltage is then converted to acceleration measurement over a calibrated voltage range, or sensitivity. Containment of signal conditioning within the ICP units promotes ease of use, low cost, and reliability in harsh environments, making them an ideal choice for this study. Selected specifications for the three types of accelerometers used in this study are given in Table 4-1.

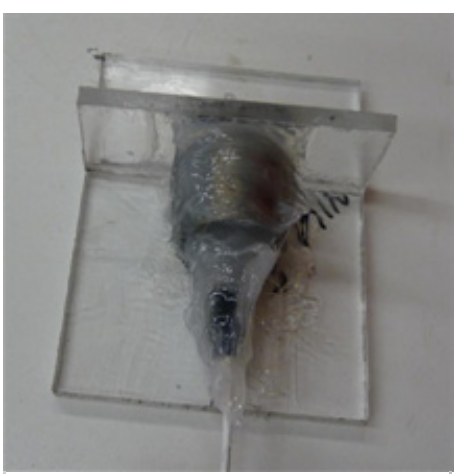

(a)

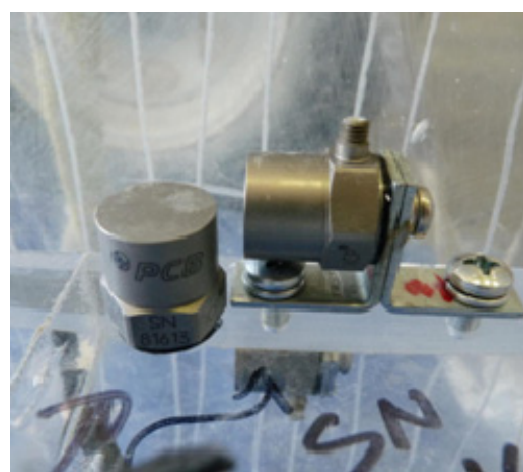

(b)

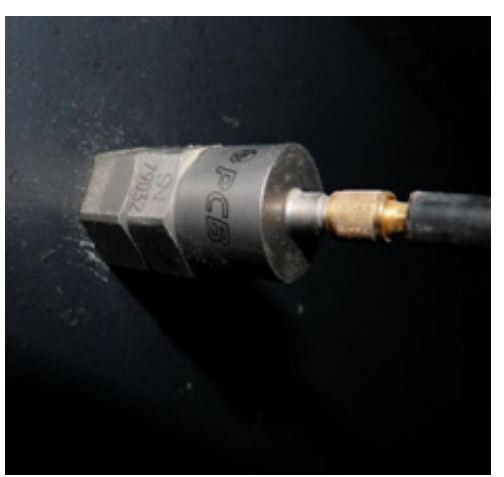

(c)

Figure 4.3 - Accelerometers used in this test program: (a) 393B04 ICP accelerometer mounted to and acrylic foundation and protected from moisture and chemical attack using $100 \%$ silicone (b) J353B51 ICP accelerometers mounted within model structure (c) 353B52 ICP accelerometer mounted to shake table.

Table 4-1 Selected accelerometer specifications

\begin{tabular}{|l||l|l|l|}
\hline Accelerometer & 393B04 & J353B51 & $353 \mathrm{~B} 52$ \\
\hline Model Measurement & soil & structural & table \\
\hline Quantity & 10 & 5 & 1 \\
\hline $5 \%$ Frequency range $(\mathrm{Hz})^{*}$ & 0.06 to $450 \mathrm{~Hz}$ & 1 to $2000 \mathrm{~Hz}$ & 1 to $2000 \mathrm{~Hz}$ \\
\hline Sensitivity $\mathrm{mV} / \mathrm{g}$ & 1000 & 500 & 500 \\
\hline Housing & titanium & titanium & titanium \\
\hline Piezoelectric Material & ceramic & quartz & quartz \\
\hline Sensing Geometry & flexural & shear & shear \\
\hline
\end{tabular}

* Flat response frequency range with $\pm 5 \%$ sensitivity deviation

The operational frequency of the accelerometers is the frequency range in which the voltage output sensitivity results in a flat response in relation to the acceleration input. 
Referring to Table 4-1, the 393B04 accelerometers are better suited for low frequency seismic applications. Also, the higher sensitivity means that the output resolution will be better in relation to the noise level of the signal conditioning. The two quartz shear accelerometers are adequate provided that the important frequency content of the model response is higher than $1 \mathrm{~Hz}$. Output is reduced at frequencies lower than $1 \mathrm{~Hz}$.

The 393B04 accelerometers placed in saturated clay needed to be fully protected from moisture and chemical attack, and the hermetic titanium encasements that house the ICP units fit this criterion well. A single coaxial electrical connection is the only place where moisture can penetrate the units. This connection was sealed using $100 \%$ silicone sealant. The soil accelerometers were attached to individual foundations fabricated from acrylic plastic for the purpose of minimizing movement and rotation within the soil mass under seismic loading. The flat surfaces on the foundations also help with properly aligning the units within the soil. Weld-On 16, distributed by TAP Plastics, was used to form the foundations by orthogonally joining $75 \mathrm{~mm}$ by $30 \mathrm{~mm}$ vertical cards to $75 \mathrm{~mm}$ by $90 \mathrm{~mm}$ base cards (Figure 4.3a). Silicone sealant was applied to cover the entire accelerometer to protect the casing from chemical attack from the soil.

The soft, cohesive properties of the model soil complicated accelerometer placement and positioning. Tools employed in placement included plum bobs and timber beams for positioning and small levels for aligning accelerometers on axis. After placement and burial, it is not possible to verify the position or angle of the soil accelerometers. Small positional or angular changes are possible during testing, but under the expected low-strain conditions, this is not a significant problem. Extreme care 
should be taken during model construction, as this is when the accelerometers are the most susceptible to disturbance.

Reduced output signal may result from accelerometers not being perfectly aligned on axis. Approximately $1.5 \%$ signal reduction is possible for 10 degree off axis alignments. This level of alignment precision is realistic using the manual placement methods described here. Accelerometer output can be expected to vary within a reasonable tolerance depending on alignment, vibration frequency, temperature response, signal noise, and transverse sensitivity, but total output variation cannot readily be characterized.

\subsubsection{Wire Potentiometers}

Wire potentiometers or "wire pots" were used to measure absolute displacements of the soil column in reference to a fixed frame mounted next to the shake table. The units house a tensioned coiled wire which induces a proportional voltage change as the length of the wire changes along its axis. Differential voltage is returned to the data acquisition system and converted to displacement in any desired measurement unit. Wire pots allow for measurements of large displacements, and the tensioned wire adds simplicity and versatility to test setup.

The units utilized in this research are model PN 62-60-8141 manufactured by SpaceAge Control Inc. and have a maximum linear range of 2.16 meters with a precision tolerance of approximately $0.51 \mathrm{~mm}$. Fishing line, swivels, and zip ties were used to attach the wires to the Spectra bands on the test container. This is not a perfectly rigid connection due to the flexibility of the testing container, which likely results in a loss of 
measurement fidelity. Test results illustrate that the data precision is not sufficient for estimating differential soil displacement with depth, but the wire pots provide good approximation of the absolute earthquake displacements. These measurements are not a priority for this research beyond providing the approximate shape of the motions induced by the shake table. Figure 4.4 shows the Phase II wire pot setup with the wires extended toward the container to the right.

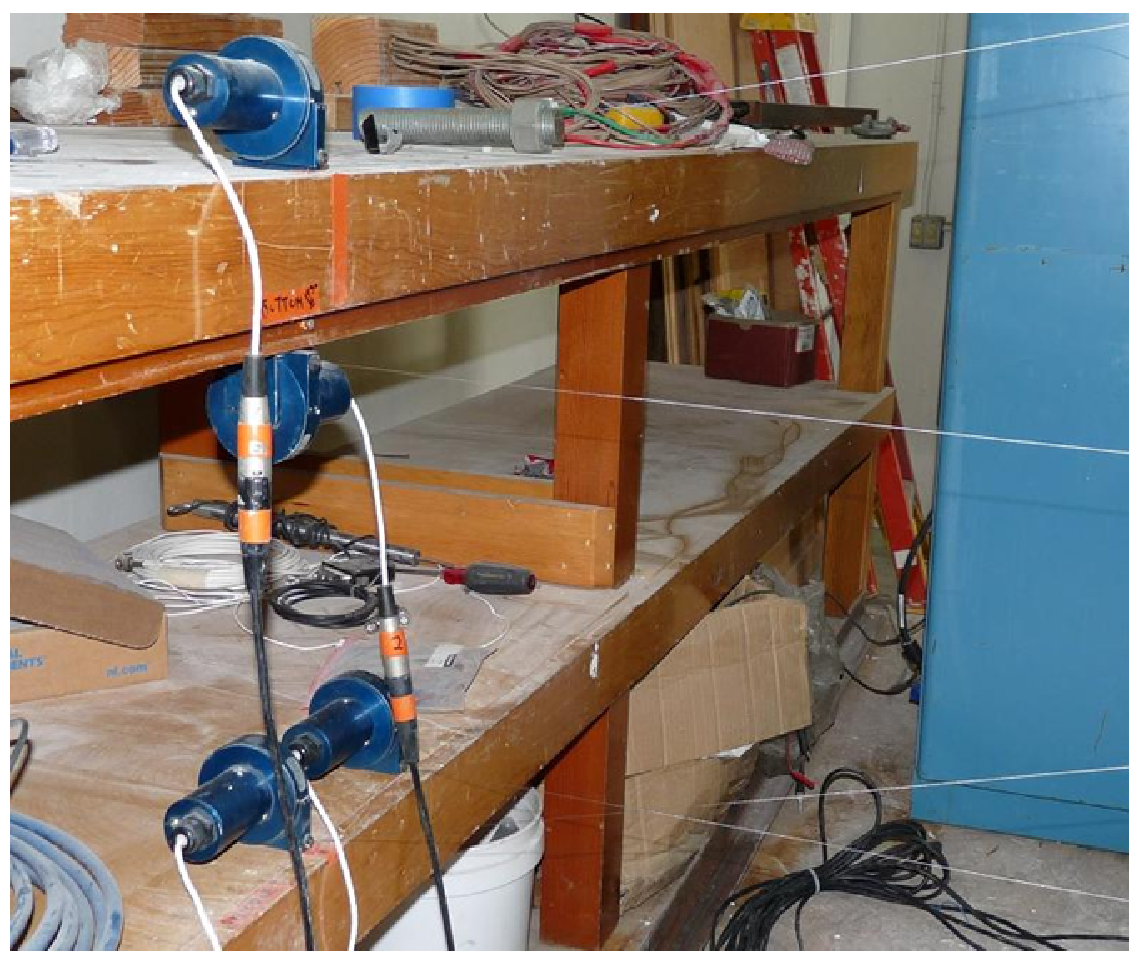

Figure 4.4 - Four wire potentiometers mounted off of shake table

\subsubsection{T-bar Penetrometer}

Stewart and Randolph (1991) developed the T-bar penetrometer device for estimating continuous soil strength with depth. Validation of the device was performed in both field and centrifuge environments. T-bar penetrometer undrained shear strength results agreed well with triaxial, vane shear, and cone penetrometer results. The T-bar 
consists of a roughened horizontal cylindrical cross bar oriented perpendicular to a narrow rod, forming a "T" shape. The rod allows the cross bar to be pulled or pushed through soil, measuring constant resistance with a load cell. Estimation of undrained shear strength from the resulting load resistance profile is based on earlier research by Randolph and Houlsby (1984). They used classical plasticity theory to derive closedform solutions for the limiting pressure acting on a circular pile moving laterally through soil. Adapted for the T-bar, the analysis assumes that soil flows around the cylinder and fully closes behind it without gapping or suction effects. Any effect from the narrow rod is ignored in the analysis due to its relatively small cross-section compared to the cross bar. Undrained strength is evaluated from the limiting force acting on an infinitely long cylinder (Meymand 1998):

$$
S_{u}=\frac{P}{N_{b} D}
$$

where $S_{u}=$ undrained shear strength of the soil, $P=$ force per unit length acting on the cylinder, $N_{b}=$ bar factor, and $D=$ diameter of the cylinder.

The bar factor is a function of the adhesion or roughness of the cylinder and ranges from 9 to 12 for adhesion values of 0.0 to 1.0 respectively. Randolph and Houlsby (1984) suggest using a bar factor of 10.5 factor for general applications. This factor was used to analyze T-bar results in this study. The T-bar consists of a $95 \mathrm{~mm}$ long, $19 \mathrm{~mm}$ diameter steel cross bar welded orthogonally to a 2.1 meter long, $6.3 \mathrm{~mm}$ diameter pulling rod. A $2.2 \mathrm{kN}$ load cell is threaded to the end of the rod to measure constant soil resistance with depth (Figure 4.5a). The load cell used for T-bar pull out testing is model SSC-500-0000, by Tovey Engineering Inc. and has a tolerance of approximately $1 \mathrm{~N}$. 


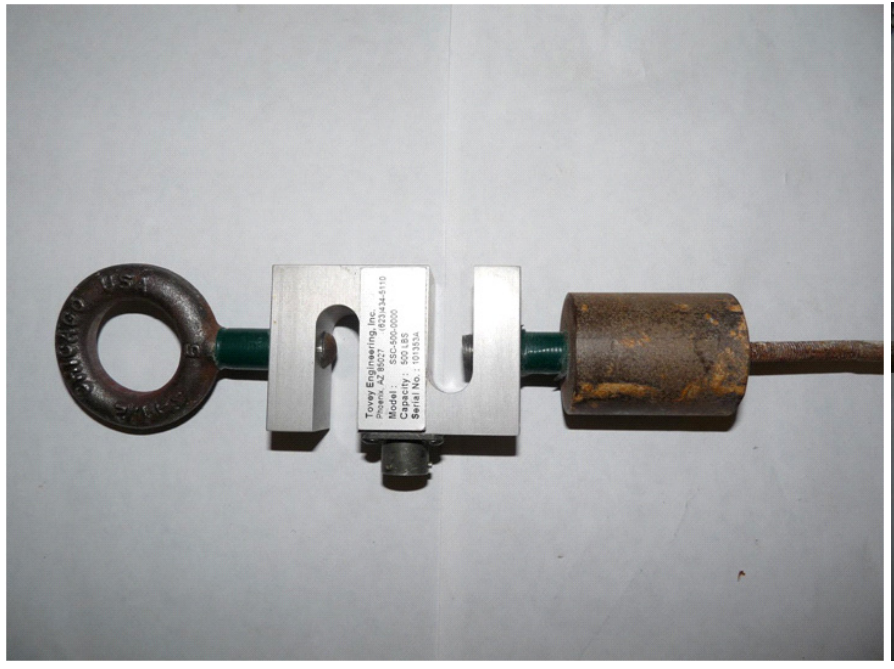

(a)

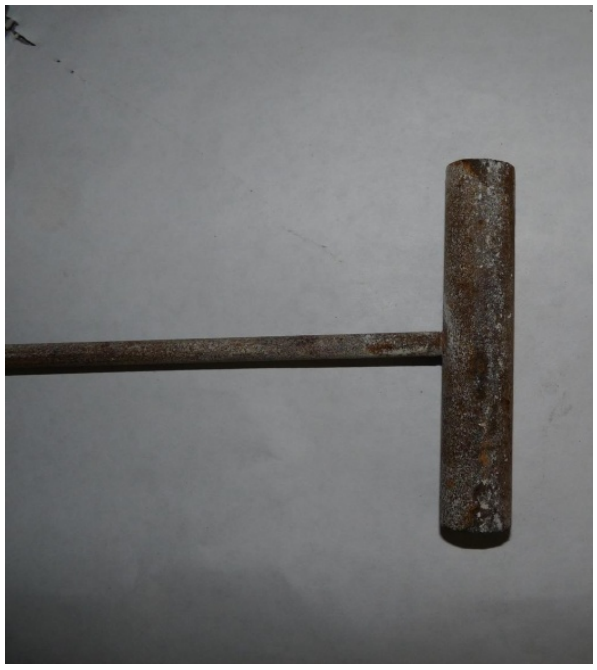

(b)

Figure 4.5 - T-bar testing device with (a) $2.2 \mathrm{kN}$ load cell threaded to T-bar rod and eye bolt for pulling and (b) T-bar penetrometer cross-bar

\subsubsection{Linear variable differential transformers (LVDT's)}

Racking deformations within the model subway were collected using LVDT's with a maximum linear displacement range of $25.4 \mathrm{~mm}$ (1 inch) and a tolerance of about 10 microns. This level of precision is well suited for measuring the small differential racking distortions expected of the stiff model subway. The device works by moving the piston through three coils contained within the cylinder. Voltage differences between the two secondary outer coils are measured based on the piston position. The center coil is a primary coil which accepts an alternating current to provide voltage in the secondary coils. When the piston is in the center position, the outer coils result in equal and opposite voltage, corresponding to a reading of zero volts. Therefore, the device measures over a range of $-12.7 \mathrm{~mm}+12.7 \mathrm{~mm}$ depending on the position of the piston. Theoretically, an LVDT is capable of measuring infinitesimally small changes in position. The resolution is only limited by the noise in the signal conditioner and wiring. 
Also, the units are very reliable, because they contain no moving parts other than the piston, which is fully isolated from the electronics ("LVDT Basics" 2003). One of two LVDT's used in this study is shown in Figure 4.6 mounted within the model and ready for testing.

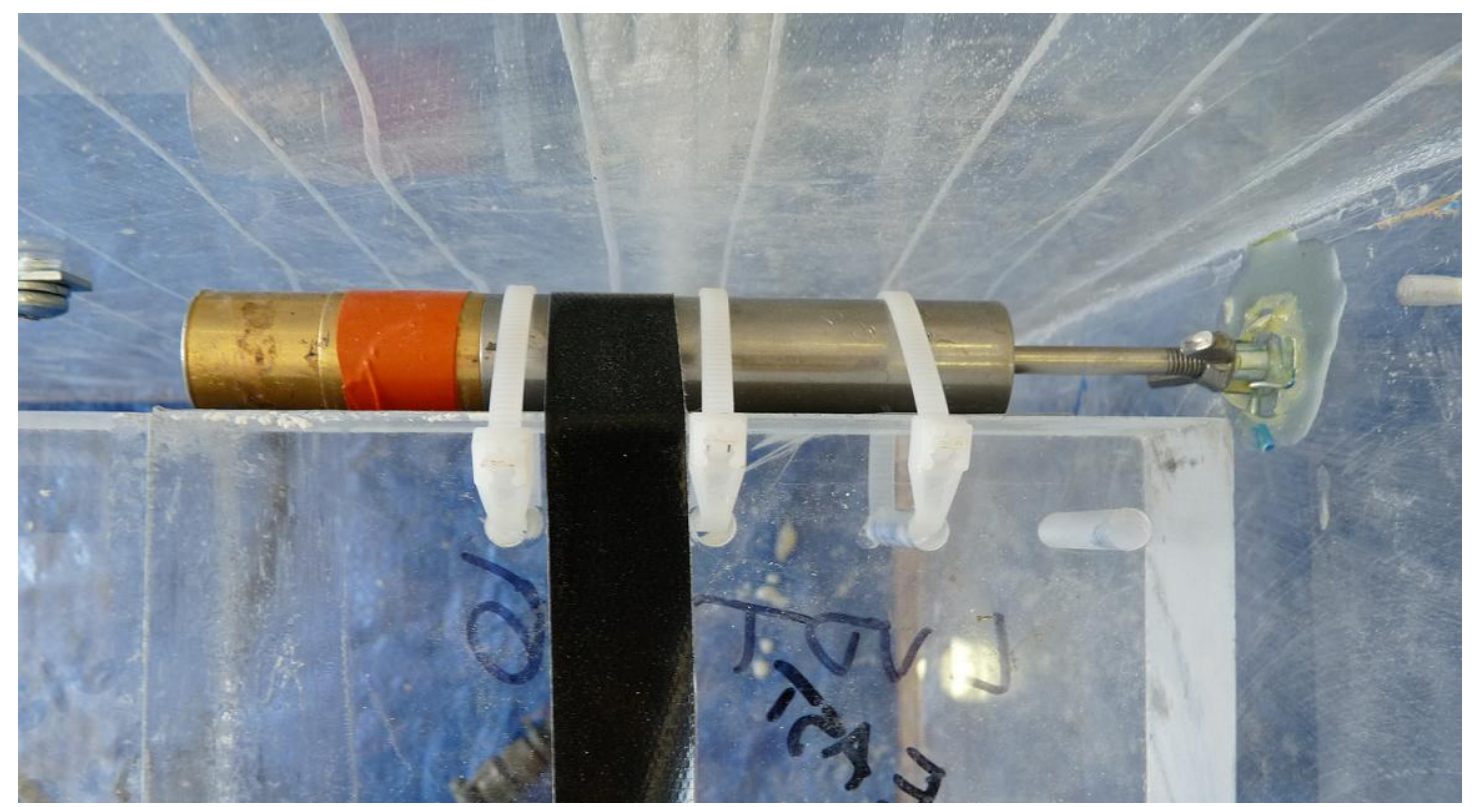

Figure 4.6 - Linear Variable Differential Transformer (LVDT) used to measure racking displacement shown mounted within model structure

\subsection{Model Soil Construction}

As described in Chapter 3, the model soil is composed of 67.5\% kaolinite, $22.5 \%$ Bentonite, and $10 \%$ class $\mathrm{C}$ fly ash by dry weight. The original model soil design in Meymand (1998) called for 100\% water content. Trial and error exposed the inability of the mixing equipment to sustain mixing at such water content. For this study, a final target water content of $125 \%$ was eventually chosen.

The mixing equipment was custom built for the UC Berkeley project by ChemGrout Inc. The designed was based on an earlier design by Arango-Greiffenstein 
(1971), who used a 3:1 ratio of kaolinite to bentonite near 200\% water content to explore seismic slope stability of saturated clay. The $2100 \mathrm{~kg}$ machine primarily consists of trailer for ease of transport, an externally powered 460 volt electric motor, a hydraulic progressive cavity pump fed by a 0.85 cubic meter tank with a funnel shaped bottom, $76 \mathrm{~mm}$ diameter cast iron circulation piping, and a $76 \mathrm{~mm}$ diameter discharge hose. Progressive cavity pumps are advantageous for mixing clay, because they can pump highly viscous fluids while maintaining constant volume, non-pulsating flow. The pump, model 2TJ8CDQSPEC manufactured by Tarby, can maintain flow capacities of 100 gallons per minute (GPM). Mixing action is provided by continually circulating the material through the pump. The tank feeds the pump which circulates the mix back up into the tank through the cast iron circulation piping. Upon completion of mixing, the material is discharged into the testing container through the flexible discharge hose. A photograph and detailed schematic of the mixer are provided in Figure 4.7 and Figure 4.8 , respectively.

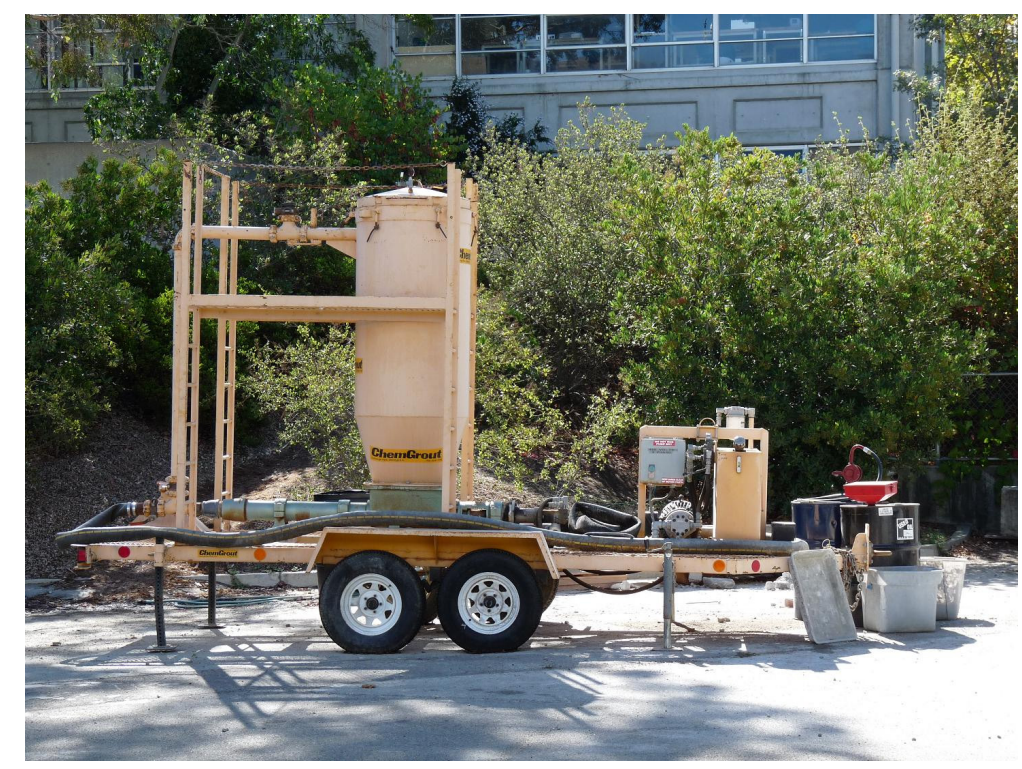

Figure 4.7 - ChemGrout mixer used to mix model soil 


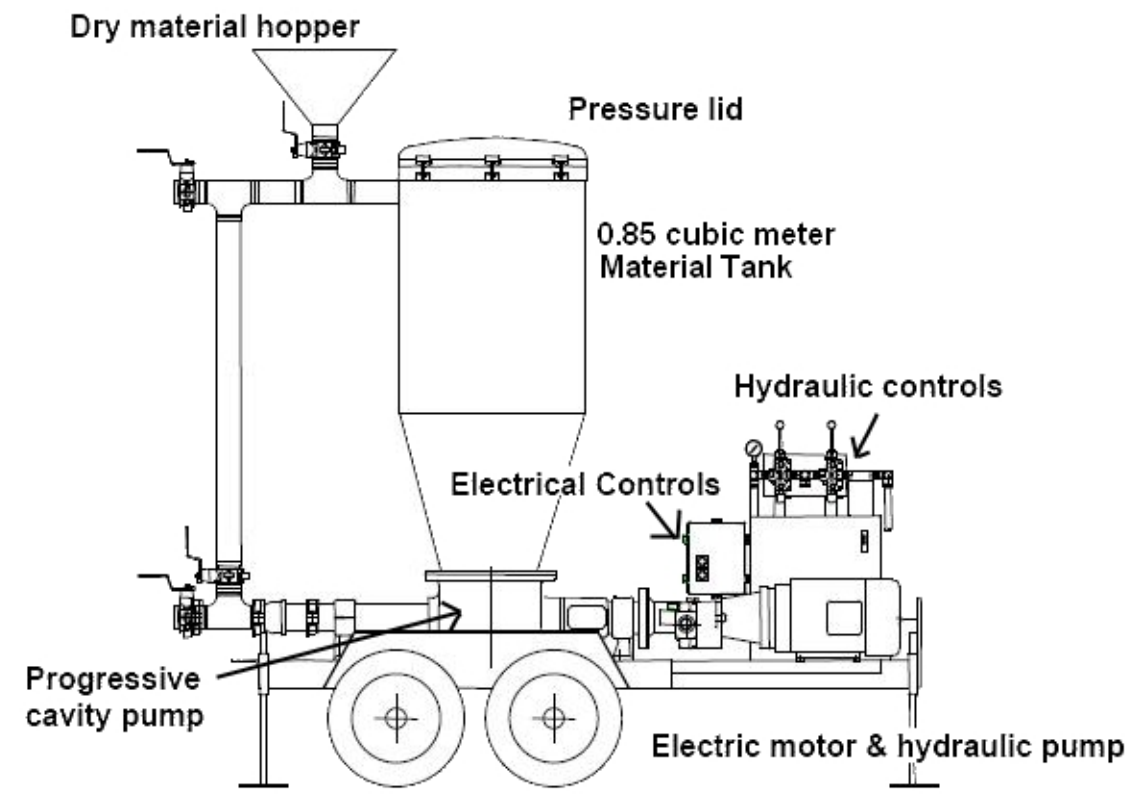

Figure 4.8 - Schematic of ChemGrout Mixer (after Meymand 1998)

The equipment is only capable of mixing a limited amount of soil at any one time, so many batches had to be prepared and packed into the test container as individual soil lifts. Water contents for the lifts range from approximately $120 \%$ to $135 \%$ due to variability in the soil batches and procedures from batch to batch. Batch size tends to vary depending on how much leftover material remains in the mixer and pump after discharge. The clay has a highly cohesive consistency and is stiff enough that it does not flow upon discharge. This results in the newly discharged material forming piles of cylindrical shaped clay chunks (Figure 4.9). Within these piles are large voids, and hand packing of all of the material is required to minimize the voids and allow for homogeneous clay lifts. The procedure is non-trivial due to the limited space and the difficulty of movement within the test container. If instrumentation such as accelerometers and T-bars are placed within clay lifts, care must be taken not to disturb 
them during material packing. More detailed equipment operation and optimal mixing procedures are outlined in Appendix A.

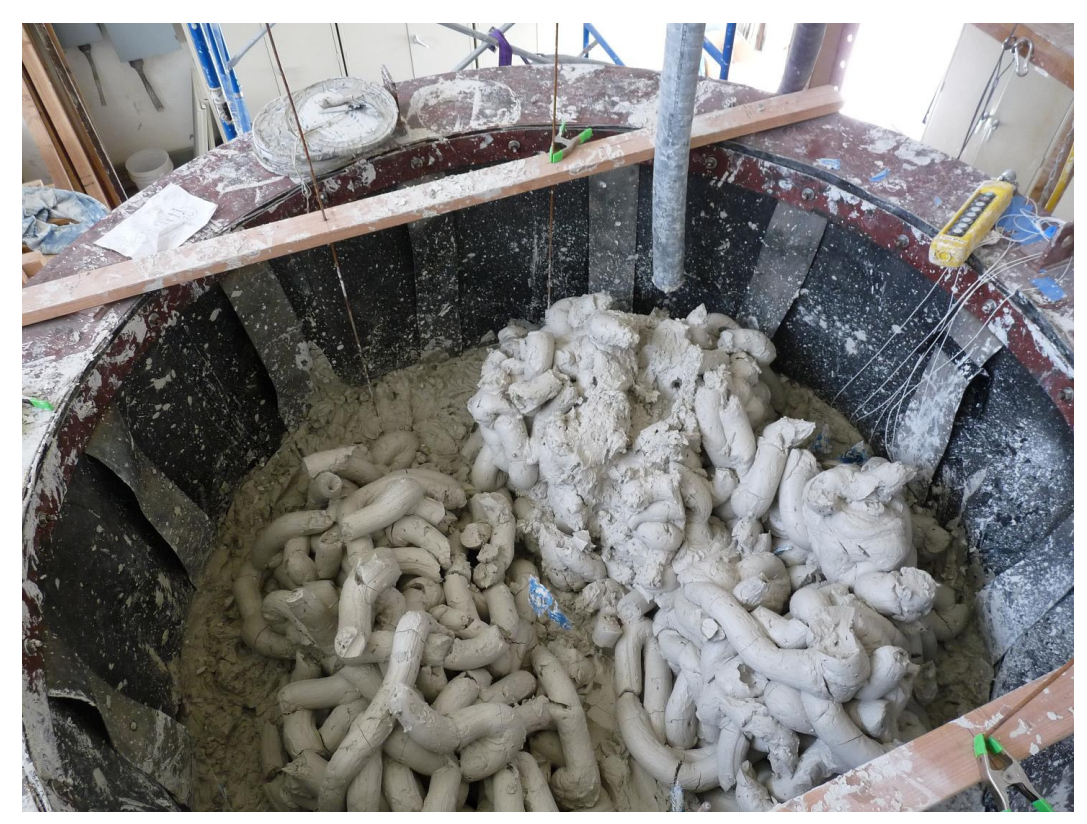

Figure 4.9 - Material immediately after discharge from mixer, ready to be hand packed

\subsubsection{Soil Batch Data}

The target soil depth for both phases of testing was 103 centimeters, corresponding to a prototype soil column depth of 10.3 meters. The base plate coupled to the shake table is analogous to underlying bedrock for the prototype. Approximately 0.45 meters of the total container height is left unfilled in order to isolate the soil column from inertial effects of the steel top ring. For both Phase I and Phase II testing, the models were built as a total of nine lifts. Not all of the lifts are of uniform depth due to variations in batch sizes. This is reflected in Figure 4.10 showing the percent contribution of each lift to the overall soil volume. 


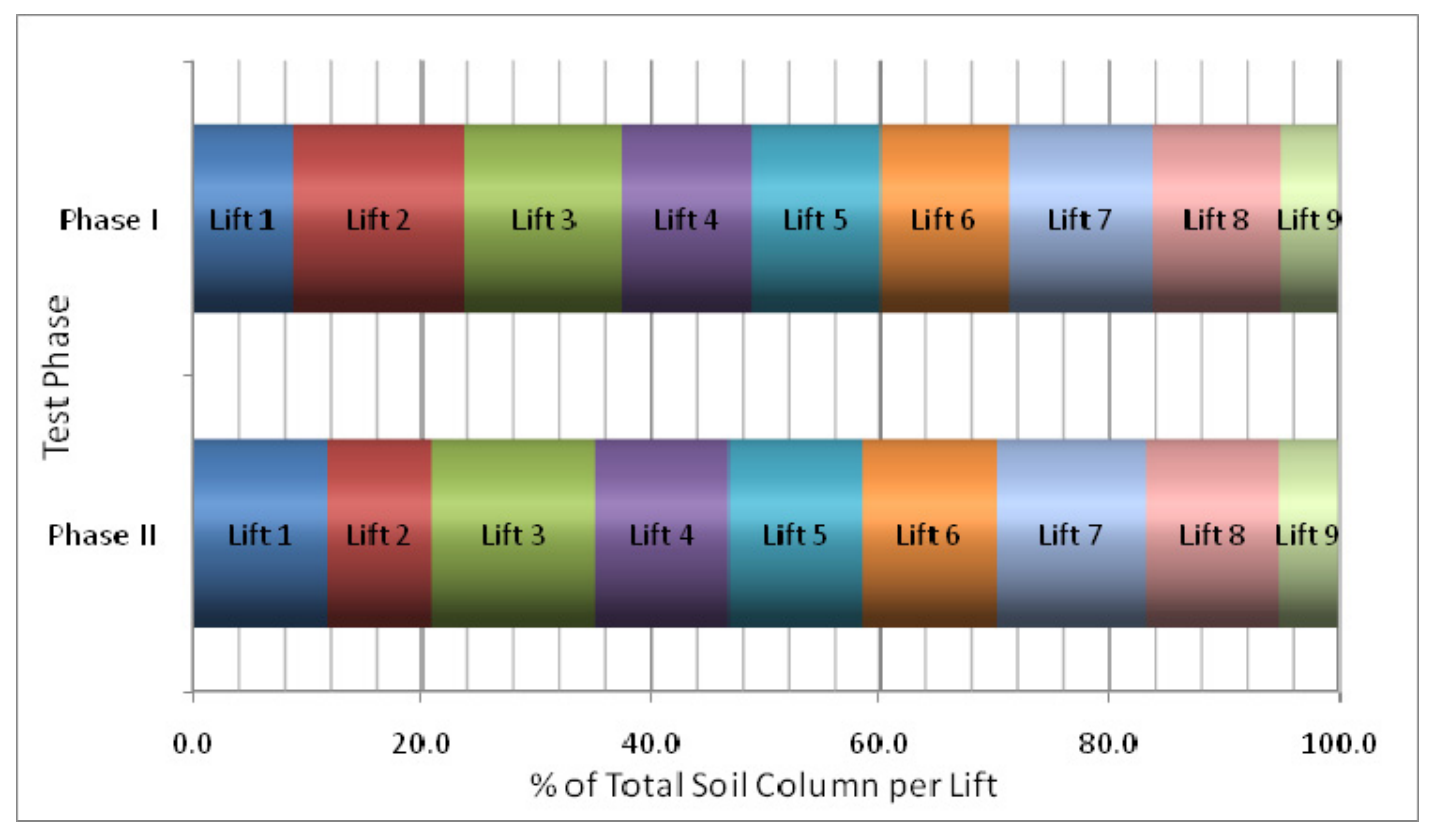

Figure 4.10 - Percent contribution of soil lifts to overall soil column volume

The first Phase I lift was placed two weeks prior to the second lift. It was mixed at a target water content of $130 \%$, and was allowed to air dry in order to form a stiff base layer. Samples were not taken to confirm water content for this base layer. Three soil samples were taken for all subsequent lifts in both phases of testing and results are shown in Figure 4.11 a) Phase I and b) Phase II. The first lift for Phase II was reconstituted from Phase I testing to form a stiff base lift. Effects of this are reflected in the inconsistency of the Lift 1 water content measurements. All other phase II lifts were composed of newly mixed model soil. Average water content for Phase II batches was generally lower than that of the Phase I soil, and T-bar test results reflect this trend, showing significantly greater soil resistance for Phase II model soil. Overall, water content was consistently within $10 \%$ or less of the target water content. 


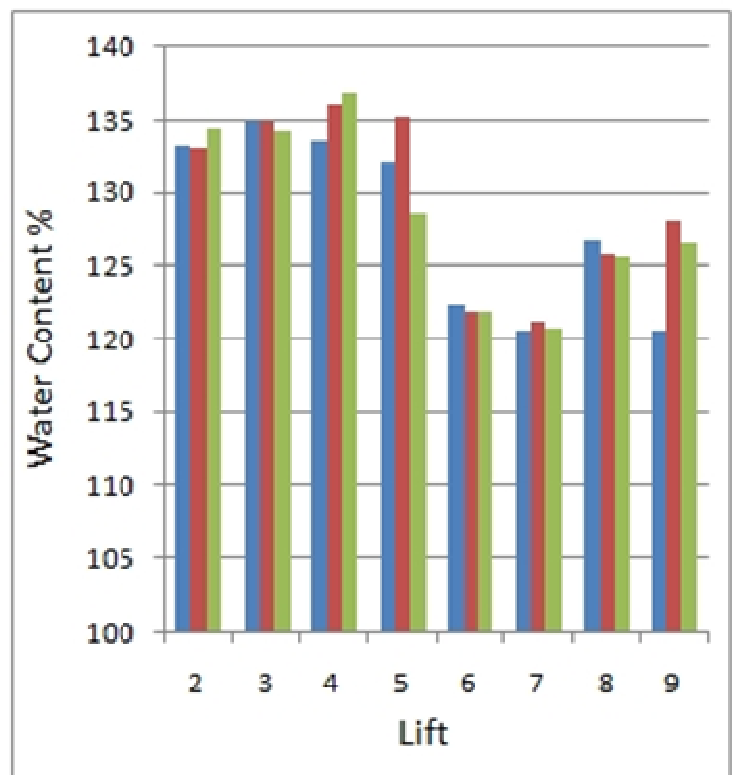

(a)

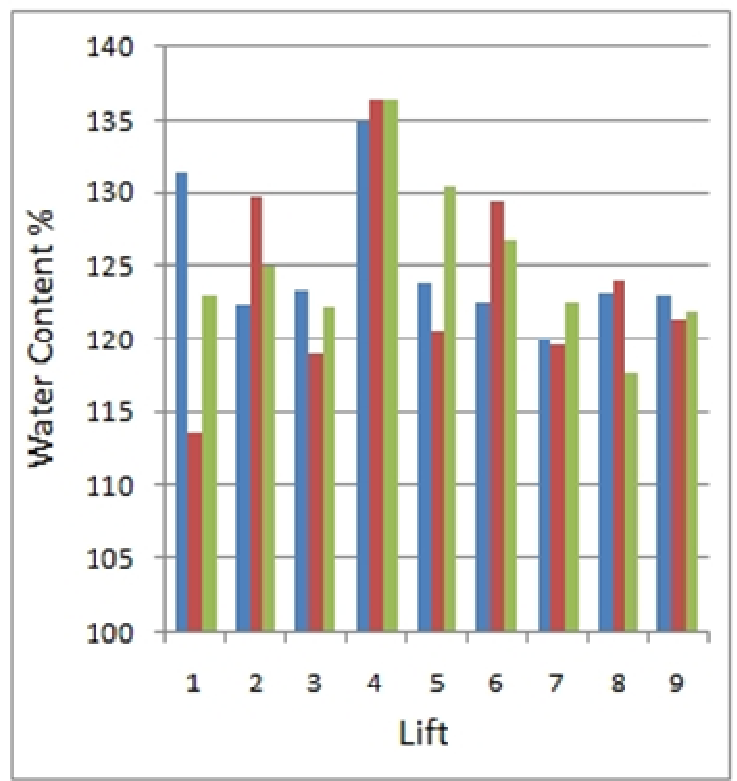

(b)

Figure 4.11 - Model soil water content at time of placement for a) Phase I and b) Phase II

Unit weight was measured for lifts 2 through 9 during Phase I, and results indicate an average unit weight throughout the soil column of just below $13.3 \mathrm{kN} / \mathrm{m}^{3}$ (Figure 4.12). This is lower than the target unit weight of $14.8 \mathrm{kN} / \mathrm{m}^{3}$ required for satisfaction of the prototype to model soil density scaling relation of 1:1. This average unit weight results in an actual scale factor of 1.11 which is close enough to unity that it should not significantly distort the model. The lower unit weight of the model soil can be attributed to the increase in water content beyond the original design water content of $100 \%$. This trend is made apparent by comparing Figure $4.11 \mathrm{a}$ and Figure 4.12, illustrating that unit weight is generally lower for lifts containing higher water content. Considering the minor variation in unit weight as a function of water content, the average unit weight of $13.3 \mathrm{kN} / \mathrm{m}^{3}$ is adopted in all data analyses for both test phases. 


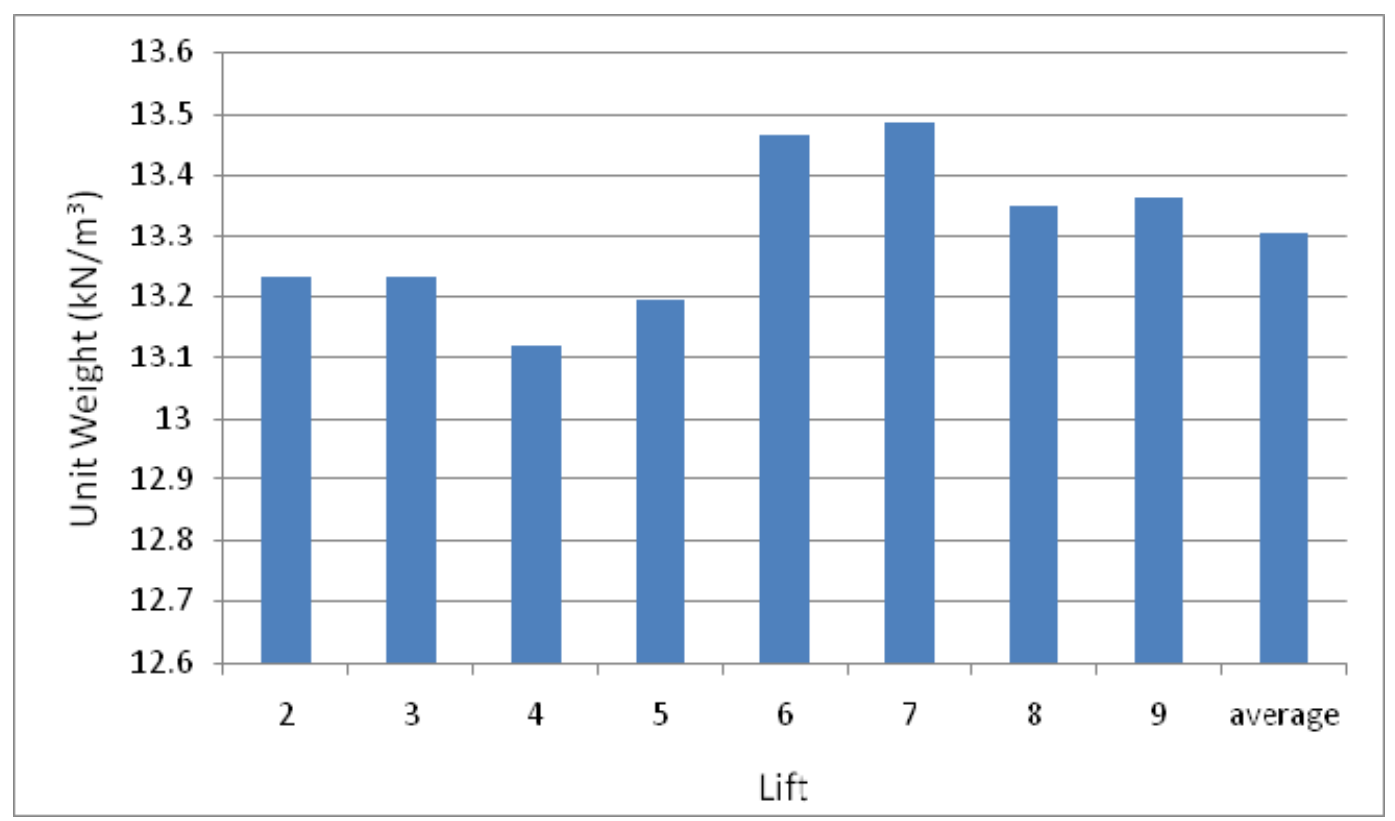

Figure 4.12 - Unit weight measurements for as placed Phase I model soil units

\subsubsection{Phase I Reconstituted Soil}

During the first day of Phase I testing, a shake table malfunction in combination with possible operator error, caused rapid jolts to the free-field soil. At this point, all external cross-bracing had been removed, and the result of the jolt was significant shear failure of the free-field soil test setup. Shown in Figure 4.13 is a view of the deformed test container viewed orthogonal to the shaking direction. The flexible wall system did not fail and only minor repairs had to be made. All of the soil had to be subsequently removed and reconstituted into the testing container. New soil did not need to be mixed, as care was taken to minimize soil loss during the process. A total of nine water content samples were taken, three each at three equally spaced soil depths during reconstitution. This sampling revealed average water content of $126 \%$, very near the target mixing water content of $125 \%$. Time was allowed after reconstitution to erase some soil strength loss before commencing shake table tests. 


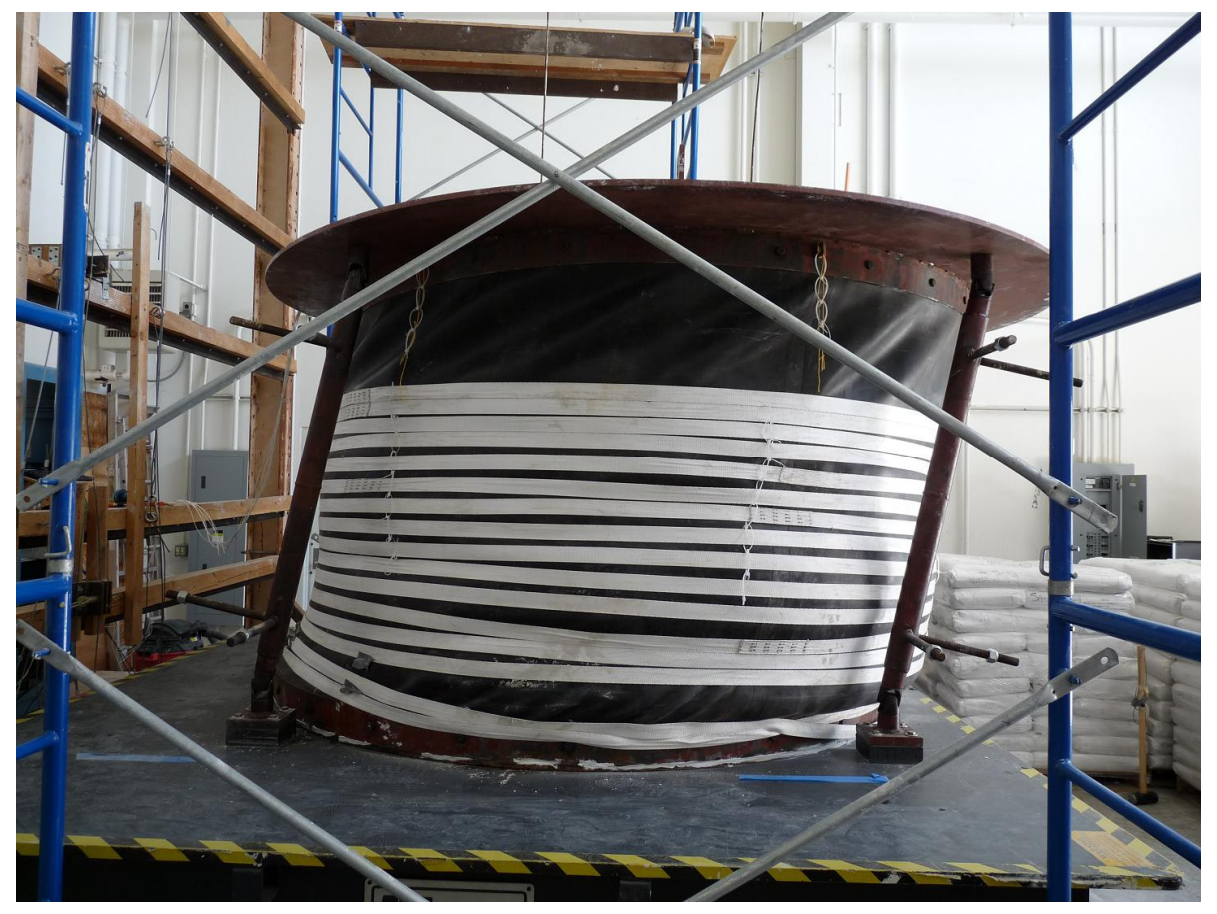

Figure 4.13 - Deformed Phase I free-field model before soil reconstitution

\subsection{T-Bar Pull-out Testing}

T-bars were placed vertically in the container prior to filling with model soil. This allows for measurement of the strength of the full soil profile. Four T-bar pull out tests were performed for each phase of testing, allowing for characterization of soil strength gain overtime and spatial variability within the soil column. Timber cross beams with drilled holes were clamped to the top ring of the container for positioning the T-bars, and also to keep them stable and upright during the test setup. The T-bars no longer require external stabilization when the container is filled with clay.

During testing the T-bar is pulled out of the soil at a constant rate of $1.29 \mathrm{~cm} / \mathrm{s}$ using the overhead crane. The pulling rate was determined by the slowest constant speed for the crane. Knowing the constant pulling rate and soil depth eliminates the need to take positional measurements during testing. A load cell was fixed between the threaded 
rod of the T-bar and the crane, and load data was recorded at a sampling rate of $25 \mathrm{~Hz}$ for the full depth of T-bar embedment. In Phase I the T-bar was placed on top of a stiffer base layer of $10 \mathrm{~cm}$ depth, so data for the base layer is not available. The T-bars were embedded the full depth of the soil column for Phase II testing. Penetrometer rate effects are discussed with T-bar results in Chapter 5.

\subsection{Hammer Blow Testing}

Hammer blow testing was conducted for the purpose of estimating the in-situ shear wave velocity of the model soil column. Testing procedures evolved as a result of extensive trial and error. Original shear wave velocity testing was conducted by striking the base plate of the container and tracking the vertical wave propagation through an array of five accelerometers in the center of the soil column. Shear wave velocity can be computed by knowing the distance between accelerometers and the differential travel time of individual shear waves detected by the accelerometers. Accurate interpretation of the waveforms proved difficult due to wave interference and possible soil-container interaction effects. Also, the original sampling rate of $200 \mathrm{~Hz}$ was much too low to characterize the high frequency wave forms generated by the hammer blows. This sampling rate was increase to $5000 \mathrm{~Hz}$ for subsequent hammer blow testing.

The testing procedure was modified from the bottom-up method of striking the base plate to a top-down method involving striking a steel bar coupled to the soil surface, shown in Figure 4.14. Both a sledge hammer and a smaller framing hammer were used in a series of tests to investigate the effects of striking the base plate with different hammers. Testing data proved conclusive in the bottom half of the model soil column 
using the top-down method, but high frequency waves and possible surface waves distorted the data in the top portion. Similar patterns were observed by Meymand (1998) for both bottom-up and top-down hammer blow testing. The top-down method shows promise, but there is significant room for improvement for future testing including:

- cushioning hammer blows to generate lower frequency input (Meymand 1998)

- filtering hammer blow data

- increasing the density of accelerometer arrays

- couple differing trial materials to the soil surface to identify a material that maximizes clarity of wave forms

All useable data was collected after Phase I shake table testing was completed, due to the difficulties discussed here. Hammer blow testing was not performed during Phase II due to the inclusion of an embedded structure within the soil column. Results and discussion of hammer blow testing is provided in Chapter 5 .

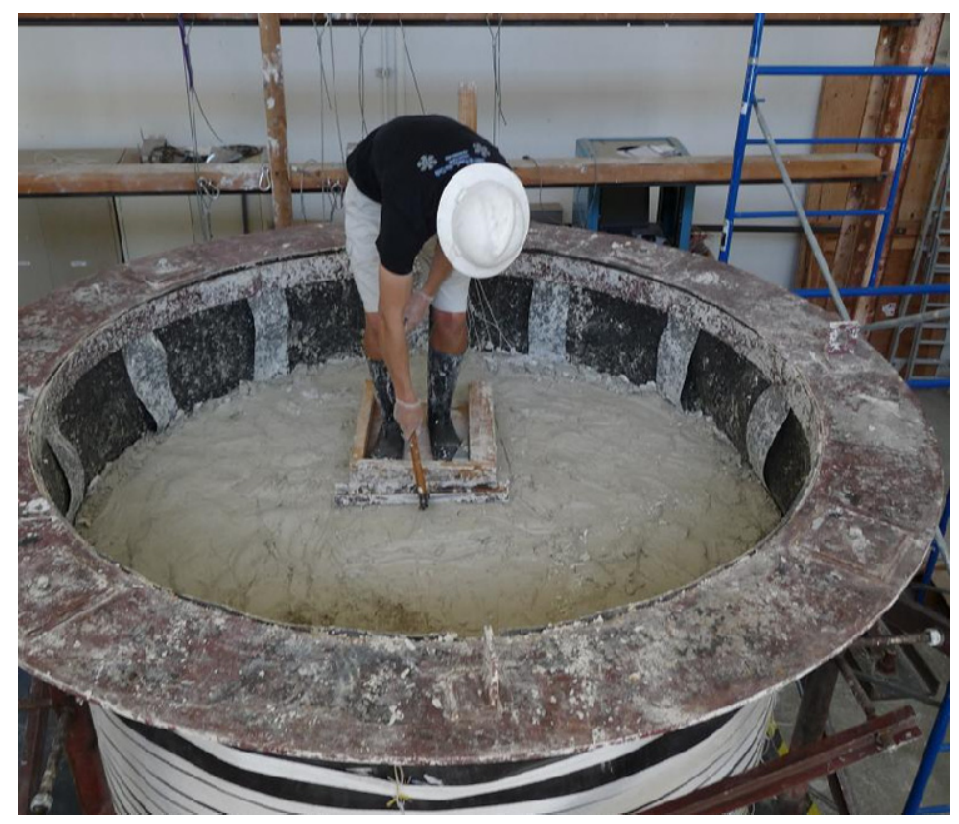

Figure 4.14 - Top-down hammer blow testing using a framing hammer 


\subsection{Shake Table Calibration}

Prior to shake table testing, it is necessary to calibrate the table response for each input motion to account for possible table-structure interaction effects. Meymand (1998) explains that "shaking table-structure interaction causes the frequency content of the table response to be altered from that of the command signal, near the resonant frequency of the test structure." This effect is most pronounced for tall structures and/or heavy structures, where significantly altered motions from those of the command signal are possible. Table calibration involves shaking the test container for a specified motion scaled to some intensity level lower than the full motion. Multiple distinct shaking iterations are required until the table responds in a manner that closely resembles the desired input motion. Due to the significant weight and non-linearity of the filled test container, motions calibrated at $40 \%$ intensity or lower insufficiently reproduced the command signal for full $100 \%$ test motions. The calibration level was increased to $65 \%$ for Phase I, and after multiple trials, a $60 \%$ calibration level was chosen for Phase II testing.

Higher calibration levels should result in more accurate table command signals, but high calibration intensity increases the risk of prematurely degrading the model soil. It is the opinion of the author that $60 \%$ calibration levels result in command signals that sufficiently match table output to the desired motion input for the filled test container. Minor differences between the desired input motion and the actual table output are not a problem as long as the actual motion is recorded. Considering the method of implied prototypes, prototypes motions may be qualitatively deduced from the actual recorded 
motion. Recorded table motions should be used for subsequent numerical analysis as the primary earthquake record, not the command input motion.

\subsection{Structural Subway Model Construction and Placement}

The purpose of Phase II testing was to investigate the USSSI effects of a rectangular tunnel cross-section embedded in soft clay subjected to transverse seismic ground shaking. Previously discussed in Chapter 3 is the design of the model according to scale model similitude. The following sections include discussions on the construction of the subway model, its unique instrumentation requirements, and placement into the model soil.

\subsubsection{Subway Model Construction}

The subway model cross-section investigated in Phase II USSSI testing was constructed entirely out of acrylic distributed by Delvie's Plastics. It is primarily composed of four $50.8 \mathrm{~mm}$ thick acrylic sheets joined on edge so that two sheets act as floor and ceiling slabs, and the remaining two sheets act as the structural sidewalls. The sheets were joined using vertical seams so that the edge of the floor and ceiling slabs contacts the inside of the sidewalls. Acrylic solvent cement (Weld-On 16) joins the sheets by softening the plastic and fusing the separate components together upon curing. If a perfect bond is achieved, it results in the separate acrylic pieces becoming a single piece of plastic. Bond quality can be inspected by examining the bond for clarity or the presence of excessive bubbles. 
Achieving a perfect bond can prove difficult because the quality of the bond is highly dependent on the quality of preparation of the bonding surfaces. The acrylic sheets were originally cut to the desired dimensions using a table saw with a specialized plastic cutting blade, but the saw cut edges tend to contain imperfections. Surface preparation for rough edges requires extensive sanding, being careful not to round the corners. Bond quality can be improved by annealing the material prior to joining, but the author was not aware of this method before construction. Weld-on 16 has an advantage over other acrylic solvents in that it is more viscous and can fill in gaps caused by rough or rounded edges, but this effect is minor and should not be relied upon. Bond quality was not perfect at some corners where bubbles had formed. To mitigate the possibility of crack propagation, small stiffeners fabricated from $6.36 \mathrm{~mm}$ thick acrylic were bonded at the corners of the inside and outside of the structure.

The tunnel cross-section is open-ended and transverse to the travel direction. Therefore, a method was developed to keep soil from caving into these open ends without significantly affecting the structural behavior of the section. Also, a watertight seal was created to protect instrumentation within the structure from moisture and chemical attack. It was important that any barrier would not significantly alter the racking characteristics of the model. A soil barrier was constructed from $6.4 \mathrm{~mm}$ thick acrylic sheets with surface area greater than the open ends of the structure. An acrylic cross-bar running through the length of the tunnel was bonded to one sheet. Upon model assembly, the cross-bar was bolted to the other sheet through the tunnel, thereby providing support against lateral soil pressure. Teflon sliders at the bottom of the barriers provided low friction contact with the floor slab, and grease was applied between the barriers and 
cross-section edges to promote sliding. A $50 \mathrm{~mm}$ diameter flexible tube was installed through one barrier to allow for instrumentation wiring to extrude from the model.

Finally, the box was loosely sealed with tape to improve moisture resistance while still allowing subway deformation. Figure 4.15 depicts the fully assembled model hanging from the overhead crane and ready for embedment into the test container.

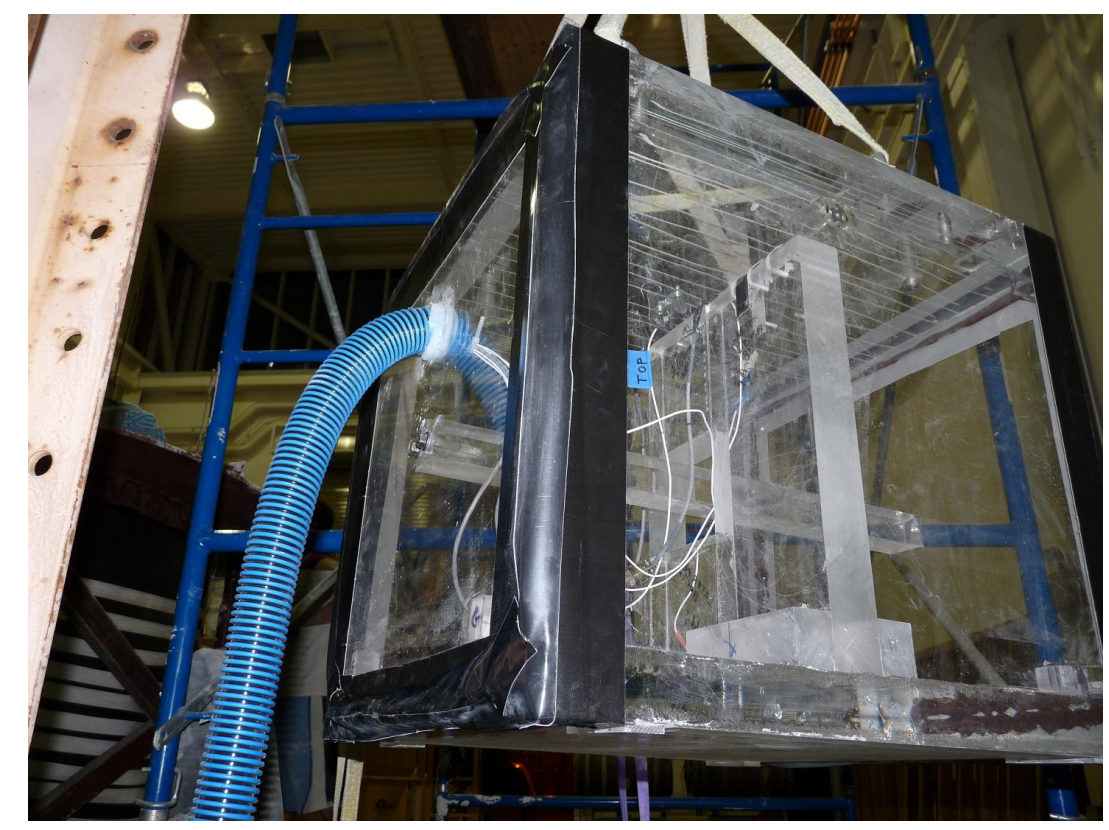

Figure 4.15 - Fully assembled model structure with soil barriers and flexible tubing to accommodate instrumentation wiring

\subsubsection{Subway Model Instrumentation}

Mounting the LVDTs to the model structure to accurately measure racking distortions was of utmost importance in the Phase II testing. Proper operation of LVDTs requires rigid mounting of the main cylinder and fixed attachment of the end of the piston to the surface of interest. Measurements along the diagonal of the structure cannot be easily achieved using LVDTs. Using tensioned wire pots to measure the diagonal 
distortions of the structure was explored as a possibility, but the resolution of the instruments available was not considered sufficient for the expected small distortions.

An internal structure within the subway was constructed for mounting the LVDTs to measure the differential racking distortions between the top and bottom slabs. A very stiff plate was constructed from $50.8 \mathrm{~mm}$ thick acrylic and mounted to the floor within the model at the midway point between the structural openings. It covers most of the area of the internal subway cross-section, leaving small gaps between the sidewalls and the ceiling slab. Differential racking deflections can be measured close to the underside of the ceiling slab in reference to the top side of the floor slab by mounting the LVDT's to the top of the plate. This does not allow for characterization of the relative distortion between the outside dimensions of the structure, but simple extrapolation can approximate the total box distortion. For redundancy, two LVDTs were used in this study to measure opposite distortions at each sidewall. A schematic of the system is shown at the end of this chapter (Section 4.10).

Double-sided mounting tape, zip-ties, and industrial strength tape were used to mount the LVDT's to the top of the plate. The LVDT pistons were securely threaded to the sidewalls using machine nuts. Simple tests were performed to check the rigidity of the coupling by physically pushing on the LVDT, which verified sufficient positional stability. A rectangular hole was built into the center of the plate to allow unhindered positioning of the barrier cross-bar through the model.

Various threaded holes were drilled into the acrylic for mounting accelerometers within the structure. Three accelerometers were mounted horizontally using small Lbrackets to measure the acceleration in the transverse direction of the tunnel (shaking 
direction). Two accelerometers were mounted to the LVDT plate, oriented horizontally along the travel direction (orthogonal to shaking direction) of the tunnel, to characterize undesirable lateral vibration of the plate. Figure 4.16 shows accelerometers and LVDT's mounted within the model structure.

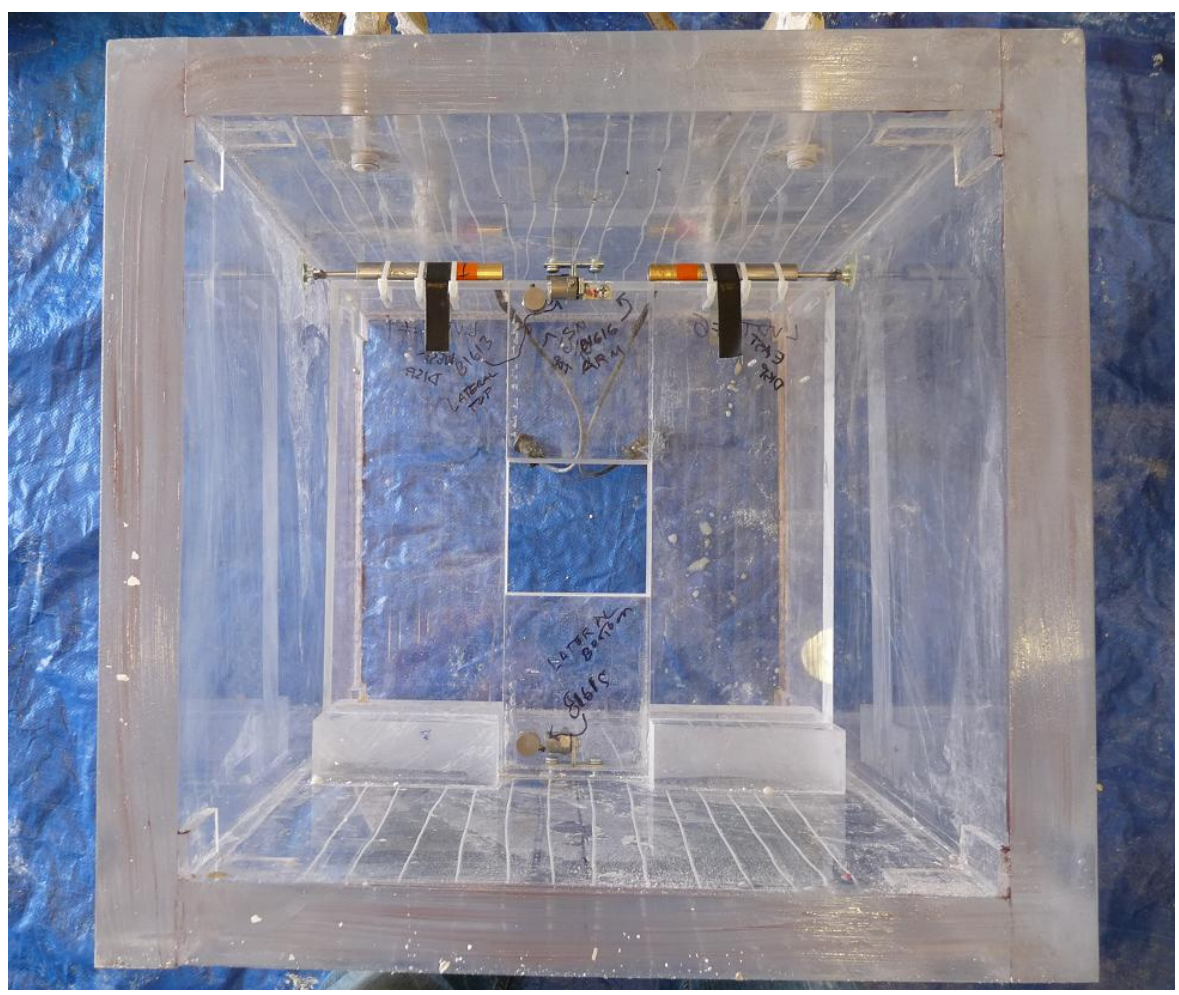

Figure 4.16 - Instrumented model structure without soil barriers

\subsubsection{Subway Embedment Procedures}

Two methods for embedment of the structure into the soil were considered.

Method one was to fully fill the bucket with model soil and excavate a cavity to install the model. The advantage of this method is two-fold: 1) it partially mimics cut-and-cover construction methods and 2) it would allow for prior characterization of the Phase II free field conditions. Method two was to place the structure at a specified depth and bury it 
during the model soil mixing and packing process. This does not mimic cut-and-cover construction practices, but it promotes better control of tunnel position and soil-structure interface contact. Method two was chosen for ease of constructability.

The depth of embedment was decided based on the minimum soil cover of 2.44 meters ( 8 feet) specified in Figure 3.3. The structure was positioned on top of 21 centimeters of soil using the overhead crane (Figure 4.17). As soil batches were mixed and discharged into the container, soil was packed around the model being careful not to disturb its position. Assuming a $103 \mathrm{~cm}$ soil column, the model was embedded with nominal soil cover of 27 centimeters. Actual soil column depth varies slightly within the test container. Upon completion of model construction, the surface of the soil was overlaid with plastic to limit desiccation at the soil surface.

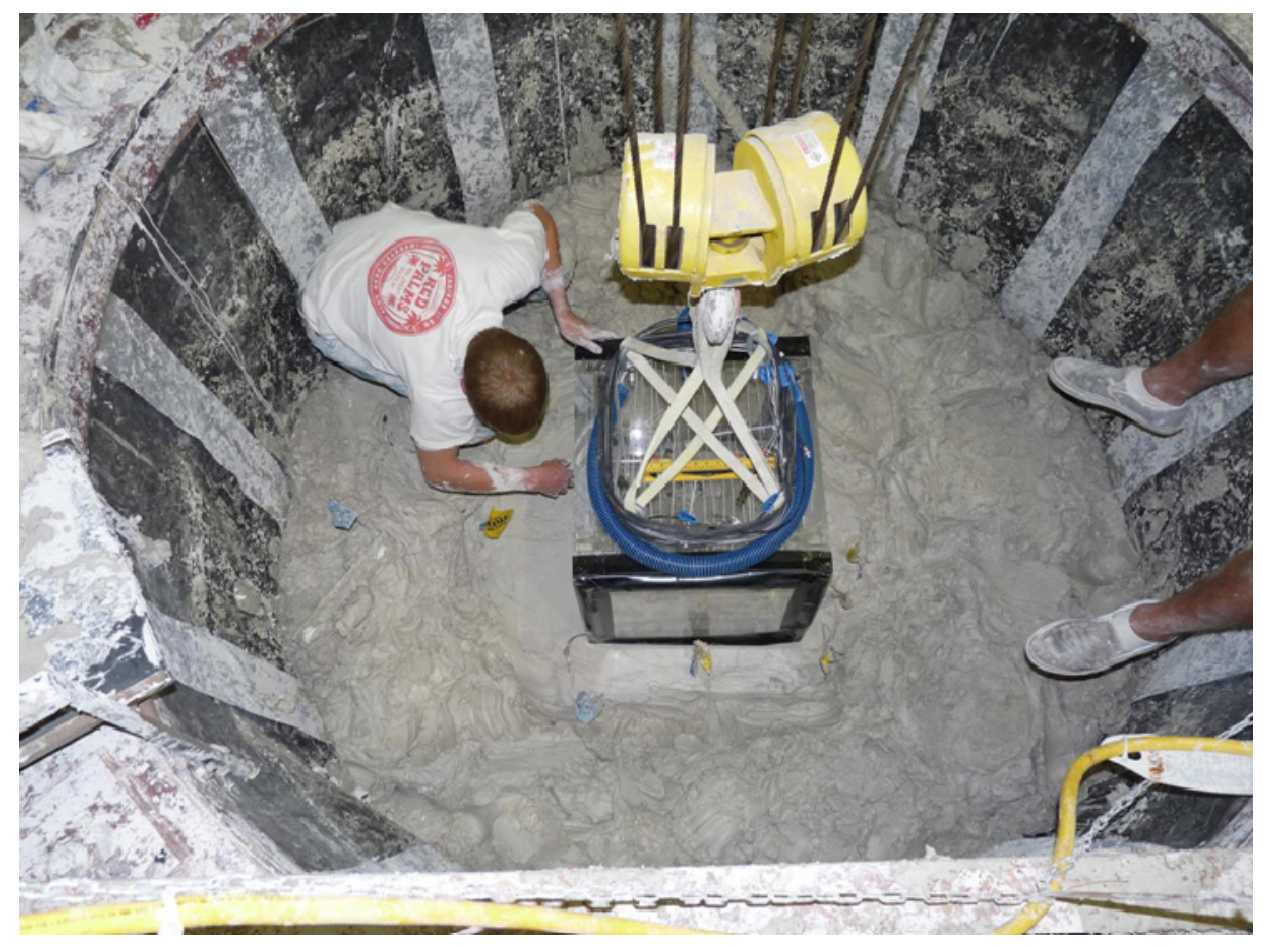

Figure 4.17 - Positioning of subway model in testing container using the overhead crane (shaking direction in photograph is approximately left to right) 


\subsection{Scale Model Testing Schedule}

Phase I free-field shake table testing commenced through June 2009 over three primary testing days. Phase II USSSI testing commenced between November and December 2009. Testing days were usually separated by three days or more to allow the soil time to regain strength loss during testing, except for a single instance of back to back testing during Phase II. Some motions were repeated due to the table operation learning process and trial and error in creating proper shake table command signals.

For example, the Phase I TCU075W test intensity needed to be lowered from $100 \%$ to $90 \%$ because the $100 \%$ TCU $075 \mathrm{~W}$ motion exceeded the table limits of displacement. The cause of this is thought to be a result of operator error in which the incorrect command signal was applied. Phase II testing was conducted by running motions at $50 \%$ and $100 \%$ intensity levels, but different trial shaking intensities were used to create table command signals, leading to a wide range of table output. These issues did not cause significant concern since the table motions were recorded and treated independent from the original input motions for subsequent analysis.

Much experience was gained from the testing process and recommended procedures were eventually developed. As a result of the extensive trial and error, not all of the recorded data was considered for rigorous data analysis. All scale model tests are reported below and data is archived for future analysis and data mining, but only pertinent test results are discussed in following chapters. Table 4-2 describes details of the final Phase I testing schedule, and Table 4-3 describes details of the final Phase II testing schedule. Listed in the tables are test ID's, test descriptions, the motion, and intensity used to calibrate the command signals applied for each shake table test. 
Table 4-2 Final Phase I testing schedule

\begin{tabular}{|c|c|c|c|}
\hline Date & Test ID & Test Description & Command signal \\
\hline $6 / 5 / 2009$ & 1_TSE & $\mathrm{T}$ bar - southeast section of container & \\
\hline $6 / 9 / 2009$ & 1 TNE & $\mathrm{T}$ bar - northeast section of container & \\
\hline $6 / 9 / 2009$ & $1 \mathrm{~J} 000$ & Josh000 motion at $100 \%$ intensity & Josh000 65\% \\
\hline $6 / 9 / 2009$ & $1 \mathrm{~J} 090$ & Josh090 motion at $100 \%$ intensity & Josh090 65\% \\
\hline $6 / 12 / 2009$ & 1_TSW & T bar - southwest section of container & \\
\hline $6 / 12 / 2009$ & 1_E270 & ElCentro 270 motion at $100 \%$ intensity & ElCentro $27065 \%$ \\
\hline $6 / 12 / 2009$ & $1 \_$E180 & ElCentro 180 motion at $100 \%$ intensity & ElCentro180 65\% \\
\hline $6 / 18 / 2009$ & 1_TNW & T bar - northwest section of container & \\
\hline $6 / 18 / 2009$ & $1 \_\mathrm{CN}$ & TCU075N motion at $100 \%$ intensity & TCU075N 65\% \\
\hline $6 / 18 / 2009$ & 1_CW & TCU075W motion at $100 \%$ intensity & TCU075N 65\% \\
\hline $7 / 1 / 2009$ & HB1 & Bottom up hammer blow testing & \\
\hline $7 / 14 / 2009$ & HB2 & Top down hammer blow testing & \\
\hline $8 / 31 / 2009$ & HB3 & Top down hammer blow testing & \\
\hline
\end{tabular}

Table 4-3 Final Phase II testing schedule

\begin{tabular}{|c|c|c|c|}
\hline Date & Test ID & Test Description & Command Signal \\
\hline $11 / 20 / 2009$ & 2_TSE & $\mathrm{T}$ bar - southeast section of container & \\
\hline $11 / 20 / 2009$ & $2 \_\mathrm{J} 000.1 \mathrm{a}$ & Josh000 motion at 50\% intensity & Josh000 65\% \\
\hline $11 / 23 / 2009$ & 2_J090.1 & Josh090 motion at 50\% intensity & Josh090 20\% \\
\hline $12 / 2 / 2009$ & $2 \_\mathrm{J} 000.1 \mathrm{~b}$ & Josh000 motion at 50\% intensity & Josh090 20\% \\
\hline $12 / 3 / 2009$ & $2 \_\mathrm{TNW}$ & T bar - northwest section of container & \\
\hline $12 / 3 / 2009$ & 2_E270.1 & ElCentro 270 motion at $50 \%$ intensity & ElCentro $27040 \%$ \\
\hline $12 / 3 / 2009$ & 2_E180.1 & ElCentro 180 motion at $50 \%$ intensity & ElCentro180 40\% \\
\hline $12 / 3 / 2009$ & 2_E270.2 & ElCentro 270 motion at $100 \%$ intensity & ElCentro270 40\% \\
\hline $12 / 3 / 2009$ & 2_E180.2 & ElCentro180 motion at $100 \%$ intensity & ElCentro180 40\% \\
\hline $12 / 7 / 2009$ & 2 TSW & T bar - southwest section of container & \\
\hline $12 / 7 / 2009$ & 2 CN.1 & TCU075N motion at $50 \%$ intensity & TCU075N 60\% \\
\hline $12 / 7 / 2009$ & 2_CW.1 & TCU075W motion at $50 \%$ intensity & TCU075W 60\% \\
\hline $12 / 7 / 2009$ & 2_CN.2 & TCU075N motion at $100 \%$ intensity & TCU075N 60\% \\
\hline $12 / 7 / 2009$ & 2 CW.2 & TCU075W motion at $100 \%$ intensity & TCU075W 60\% \\
\hline $12 / 11 / 2009$ & 2_TNE & $\mathrm{T}$ bar - northeast section of container & \\
\hline $12 / 11 / 2009$ & 2_ J000.2 & Josh000 motion at $100 \%$ intensity & Josh000 60\% \\
\hline $12 / 11 / 2009$ & 2_J090.2 & Josh090 motion at $100 \%$ intensity & Josh090 60\% \\
\hline $12 / 11 / 2009$ & 2_E270.3 & ElCentro 270 motion at $100 \%$ intensity & ElCentro270 60\% \\
\hline $12 / 11 / 2009$ & 2_E180.3 & ElCentro180 motion at $100 \%$ intensity & ElCentro180 60\% \\
\hline $12 / 11 / 2009$ & 2 CN.3 & TCU075N motion at $100 \%$ intensity & TCU075N 60\% \\
\hline $12 / 11 / 2009$ & 2 CW.3 & TCU075W motion at $100 \%$ intensity & TCU075W 60\% \\
\hline
\end{tabular}




\subsection{Shake Table Instrumentation Configuration}

Figures and tables in this section provide details on the instrumentation used for each phase of testing. Figure 4.18 shows the instrumentation setup for Phase I in the free field column. Figure 4.19 and Figure 4.20 depict the Phase II instrumentation for the soil and the structure respectively. All of the instruments are denoted with an abbreviation that is referred to throughout the results and discussion chapters of this paper (Table 4-4). Soil accelerometer position is denoted by a letter for the array (wall, center, middle, or offset) and a number for depth level, 1 being the deepest and 5 being the shallowest. Tbars and LVDTs are denoted by compass directions (e.g. NW is northwest). Wire pots are denoted by the vertical position of the measurement point (table, low, middle, or high). Soil column elevation is defined with the soil column base as the datum.

Table 4-4 Phase I and Phase II instrumentation listing and nomenclature

\begin{tabular}{|l|l|l|}
\hline Figure & Instrumentation & Denoted \\
\hline Phase I & 3 ACC's in the wall array: levels 1, 3, 5 & 1W, 3W, 5W \\
Soil Column & 5 ACC's in the center array: levels 1, 2, 3, 4, 5 & 1C, 2C, 3C, 4C, 5C \\
(Figure 4.18) & 1 ACC in middle position: level 3 & 3M \\
& 1 ACC in offset position: level 3 & 3O \\
& 4 T-bars in NE, SE, SW: NW section & TNE, TSE, TSW, TNW \\
& 3 wire pots (WP): low, middle, high & WPL, WPM, WPH \\
\hline Phase II & 3 ACC's in the wall array: levels 1, 3, 5 & 1W, 3W, 5W \\
Soil Column & 2 ACC's in the center array: levels 1,5 & 1C, 5C \\
(Figure 4.19) & 4 ACC's in the middle array: level 2, 3, 4, 5 & 2M, 3M, 4M, 5M \\
& 1 ACC in offset position: level 3 & 3O \\
& 4 T-bars: NE, SE, SW, NW section & TNE, TSE, TSW, TNW \\
& 4 wire pots (WP): table, low, middle, high & WPT, WPL, WPM, WPH \\
\hline Phase II & 1 ACC on ceiling slab of structure interior & CS \\
Structure & 1 ACC on floor slab of structure interior & FS \\
(Figure 4.20) & 1 ACC near LVDT arm top (shake direction) & ARM \\
& 2 ACC's lateral to shake direction on LVDT & LAL, LAH \\
& arm high and low & \\
& 2 LVDT's: east and west wall deflection & LVDTE, LVDTW \\
\hline
\end{tabular}




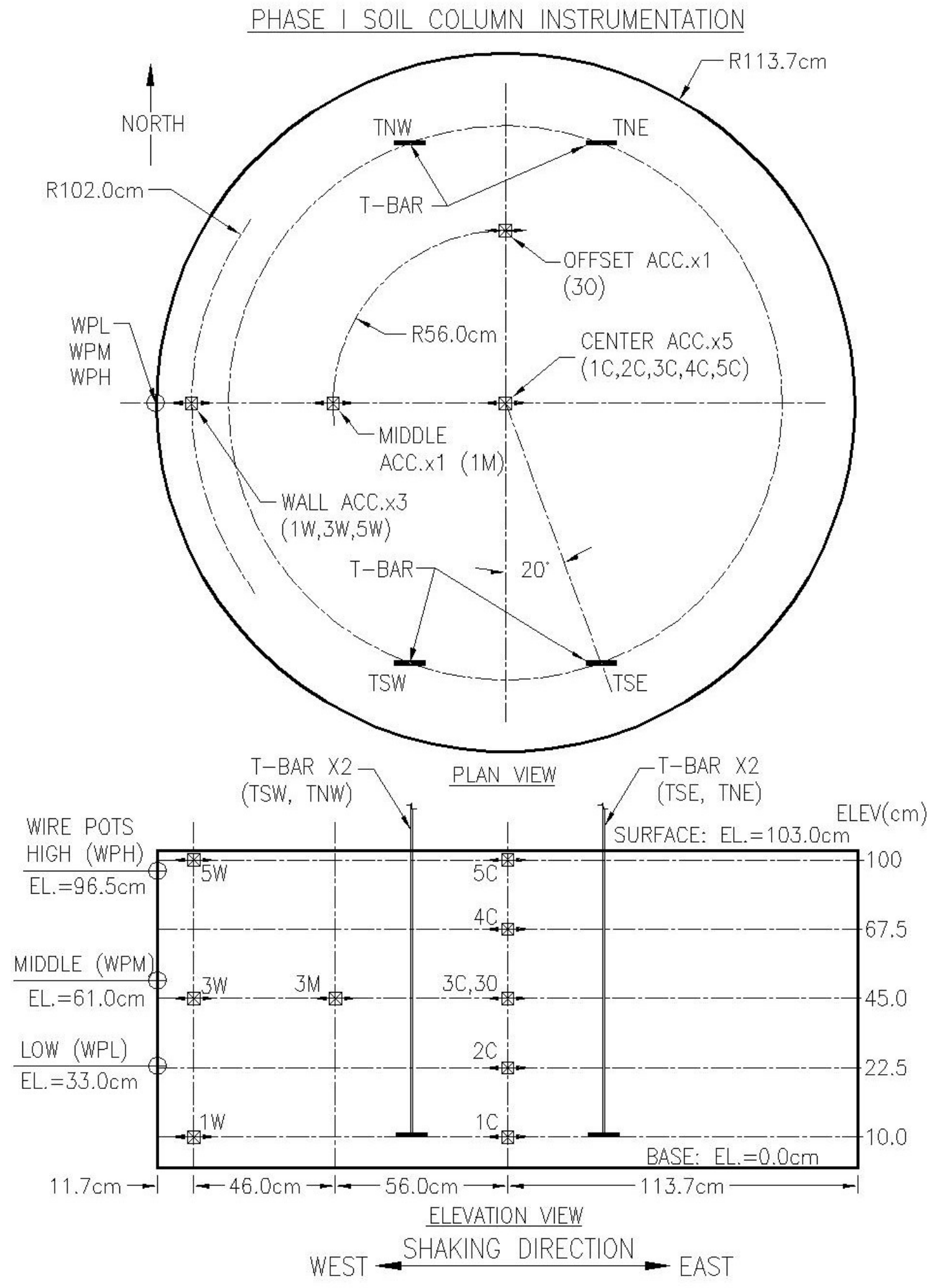

Figure 4.18 - Phase I soil column instrumentation configuration 


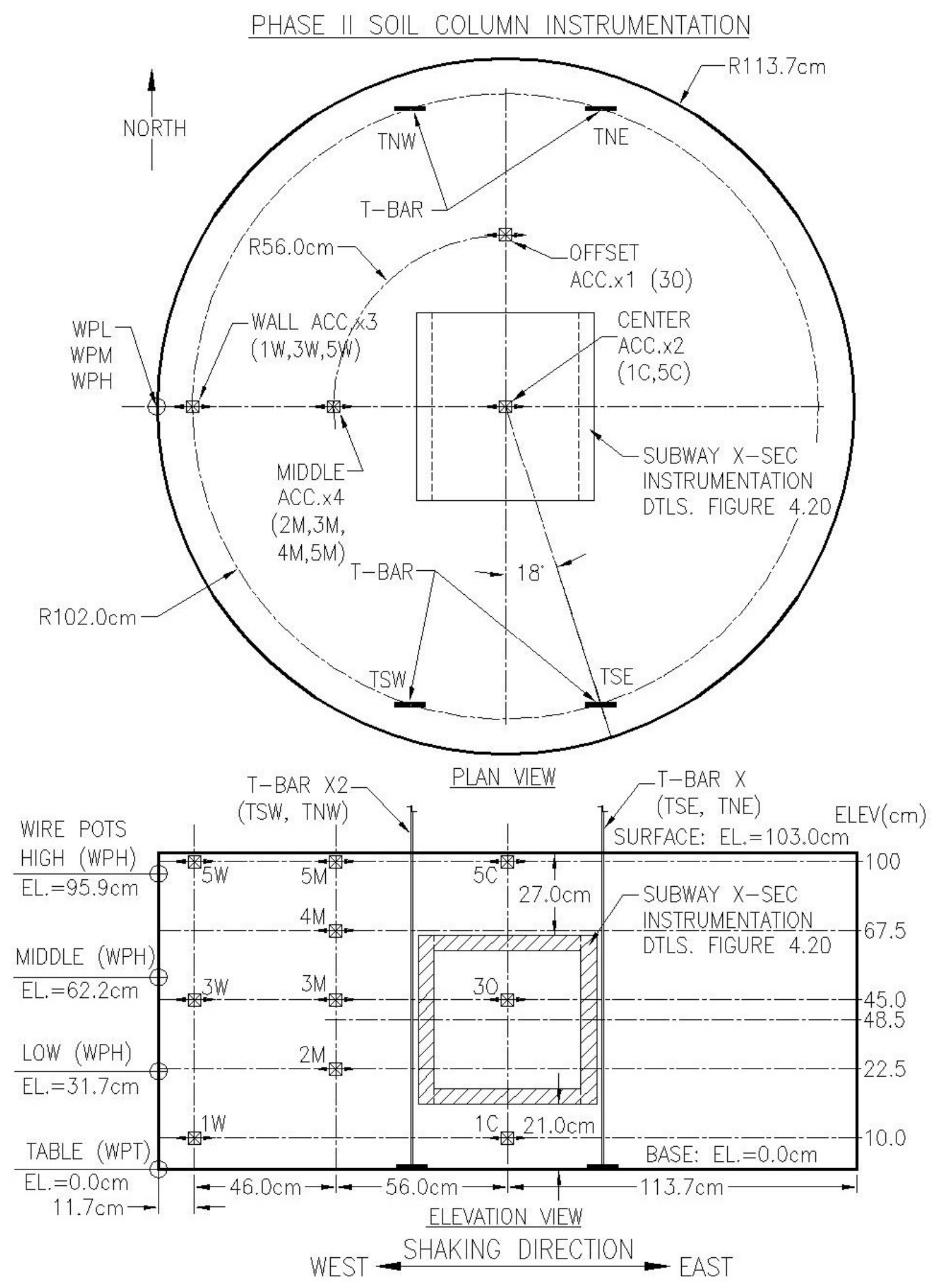

Figure 4.19 - Phase II soil column instrumentation configuration 


\section{PHASE II SUBWAY SECTION INSTRUMENTATION}
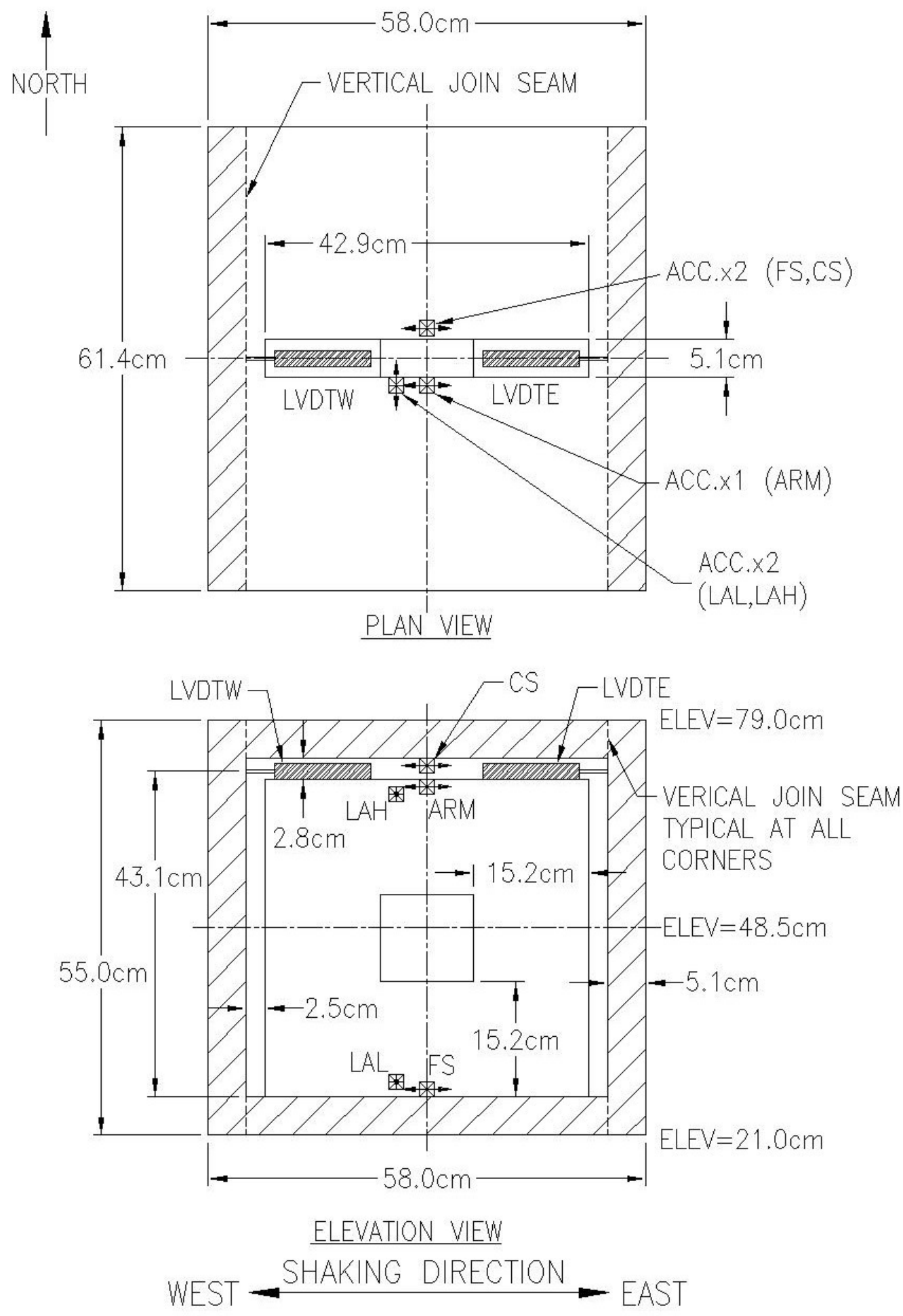

Figure 4.20 - Phase II subway cross-section instrumentation configuration 


\section{CHAPTER 5 - EXPERIMENTAL TESTING RESULTS}

\subsection{Introduction}

Test results are presented in this chapter for hammer blow, T-bar, and seismic shake table testing. Qualitative comparisons are made between particular tests for the purpose of highlighting important points regarding shake table performance and container performance. Complete experimental results are too expansive to be presented in this thesis, so an effort is made to present representative results that emphasize important details regarding soil, structural, and USSSI response.

Hammer blow and T-bar test data are considered in combination to form best estimate shear wave velocity profiles for each phase of testing. These profiles are utilized in numerical analyses using the equivalent linear codes SHAKE and FLUSH for comparison with experimental testing results. Results and discussion of numerical analyses are provided in Chapter 6. Seismic shake table testing data is mostly provided in the form of acceleration time histories and 5\% damped response spectra. Absolute displacement time histories provide displacement characteristics of the testing motions, and are compared with the desired input to qualitatively assess table performance. Structural distortion recordings are presented for Phase II testing along with a summary of maximum racking distortions for all relevant testing.

Much of the data is presented in terms of the test ID's which are listed in Table 4-2 and Table 4-3 for Phase I and Phase II tests respectively. Discussion within this chapter refers to specific instruments to denote position as well as elevation above the soil column base. The positioning scheme and associated nomenclature employed in this paper are described by the tables and figures in Section 4.10. 


\subsection{T-Bar Pullout Testing}

T-bar pullout testing was conducted to estimate undrained shear strength for the soil column in both Phase I and Phase II testing. T-bar testing results show increasing soil resistance with depth, but resistance is relatively constant through the middle of the soil column. Near surface resistance is severely underestimated due to the effect of the T-bar breaking through the soil surface. Soil resistance spikes at the bottom of the soil profile may be overestimations, a result of the overhead crane initially accelerating to a constant velocity. T-bar pull out tests were performed prior to any shake table experiments during each testing day. The test results are presented in terms of the elevation above the soil column base.

The Phase I T-bars were embedded on top of a $10 \mathrm{~cm}$ deep layer of stiffer soil, and this is reflected in the test results shown in Figure 5.1. Results show strength gain of the soil column over the thirteen day testing period. Differences between the four T-bar tests can also be attributed to spatial variation in soil strength within the soil column. Overall, the T-bar profiles show very good consistency, and it can be concluded that consolidation over the Phase I testing period was minimal.

Phase II T-bars were embedded the full length of the testing container to characterize the strength of the stiff base layer, which was composed of reconstituted soil left over from Phase I. The Phase II T-bar test results shown in Figure 5.2 illustrate spatial consistency for most of the soil profile except for the bottom $20 \mathrm{~cm}$. The soil resistance in the north side of the container is appreciably less than that of the south side. This is most likely due to the inconsistency in water content of the soil near the bottom of the profile as illustrated by Figure 4.11. The resistance variation of the Phase II T-bar 
test results is minor compared to the overall soil resistance throughout the soil column, which shows steady resistance increase with depth.

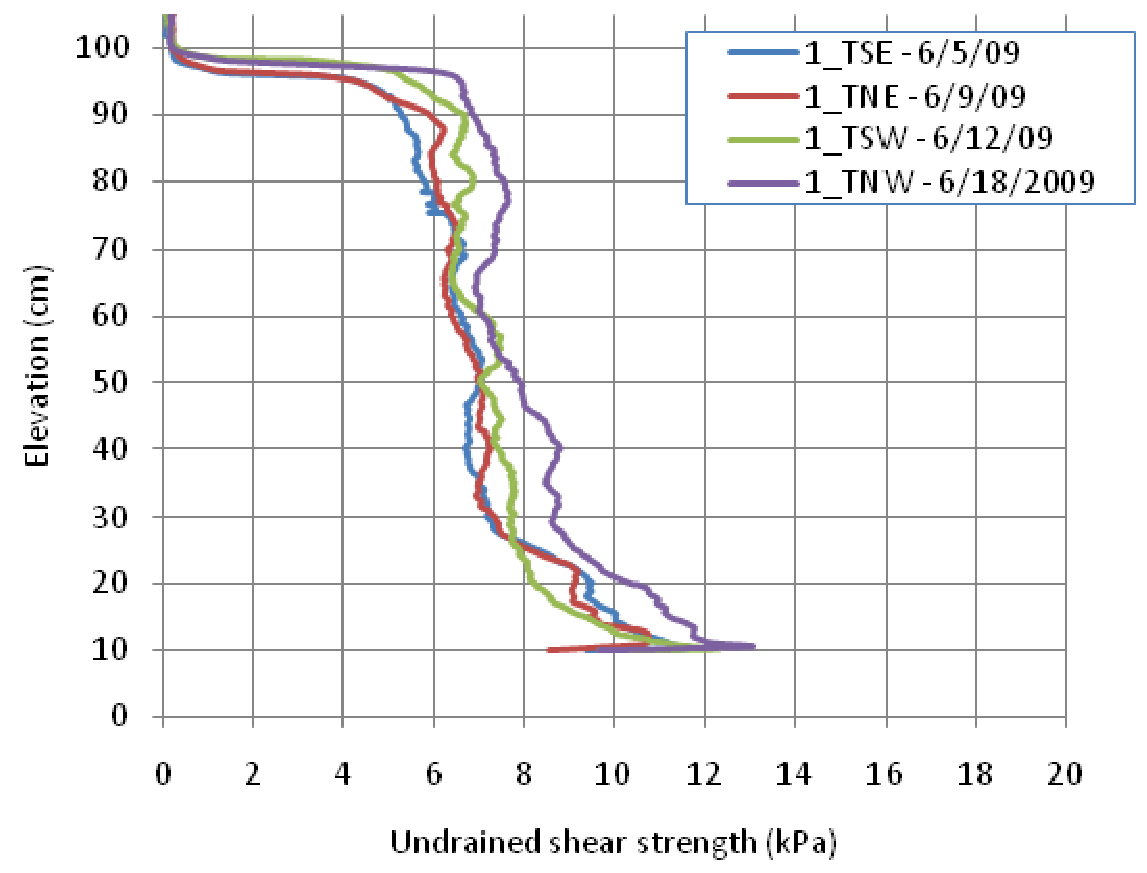

Figure 5.1 - Phase 1 T-bar testing results

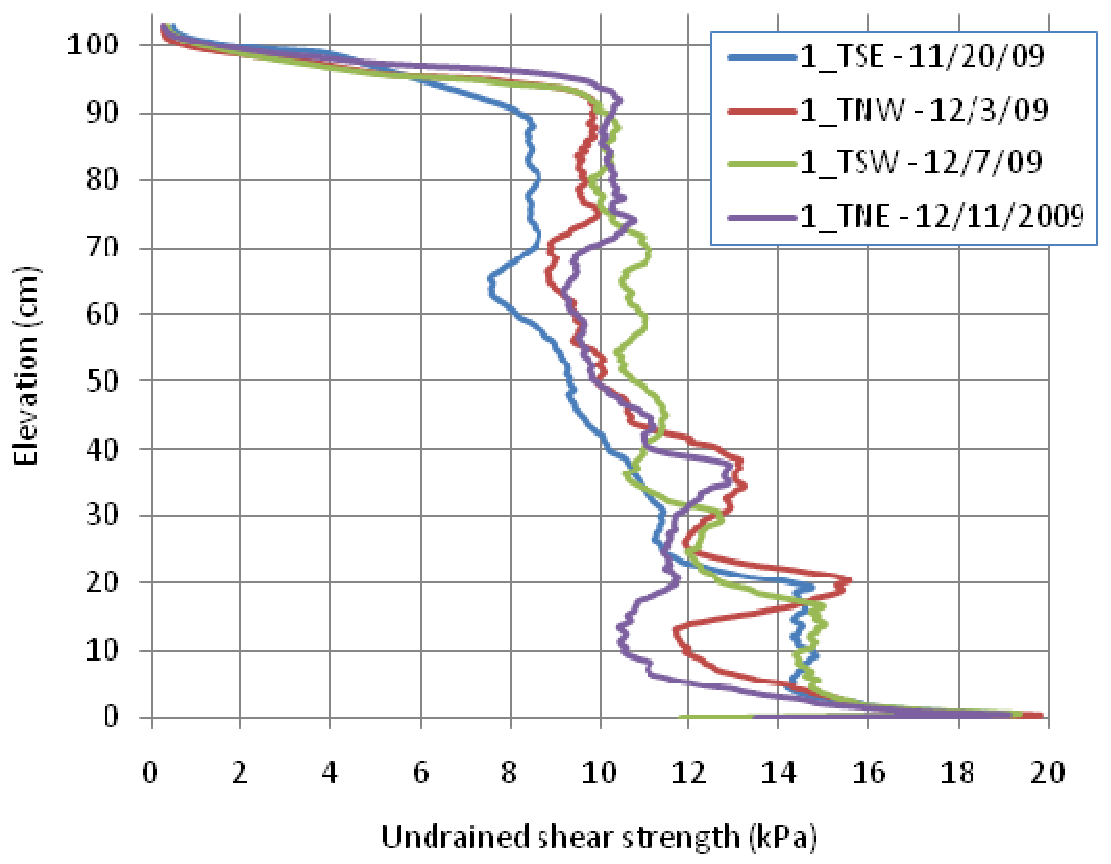

Figure 5.2 - Phase II T-bar testing results 


\subsubsection{Comparison of Phase I and Phase II T-bar Results}

The soil resistance measured from T-bar testing is greater for Phase II than Phase I. This is apparent in Figure 5.3 where average undrained shear strength profiles from both phases are overlaid on the same plot. The T-bar results are considered the most reliable between the soil column elevations of approximately $20 \mathrm{~cm}$ to $90 \mathrm{~cm}$. Over this range, Phase II soil strength is $38 \%$ to $58 \%$ greater than Phase I soil strength, according to the T-bar test results.

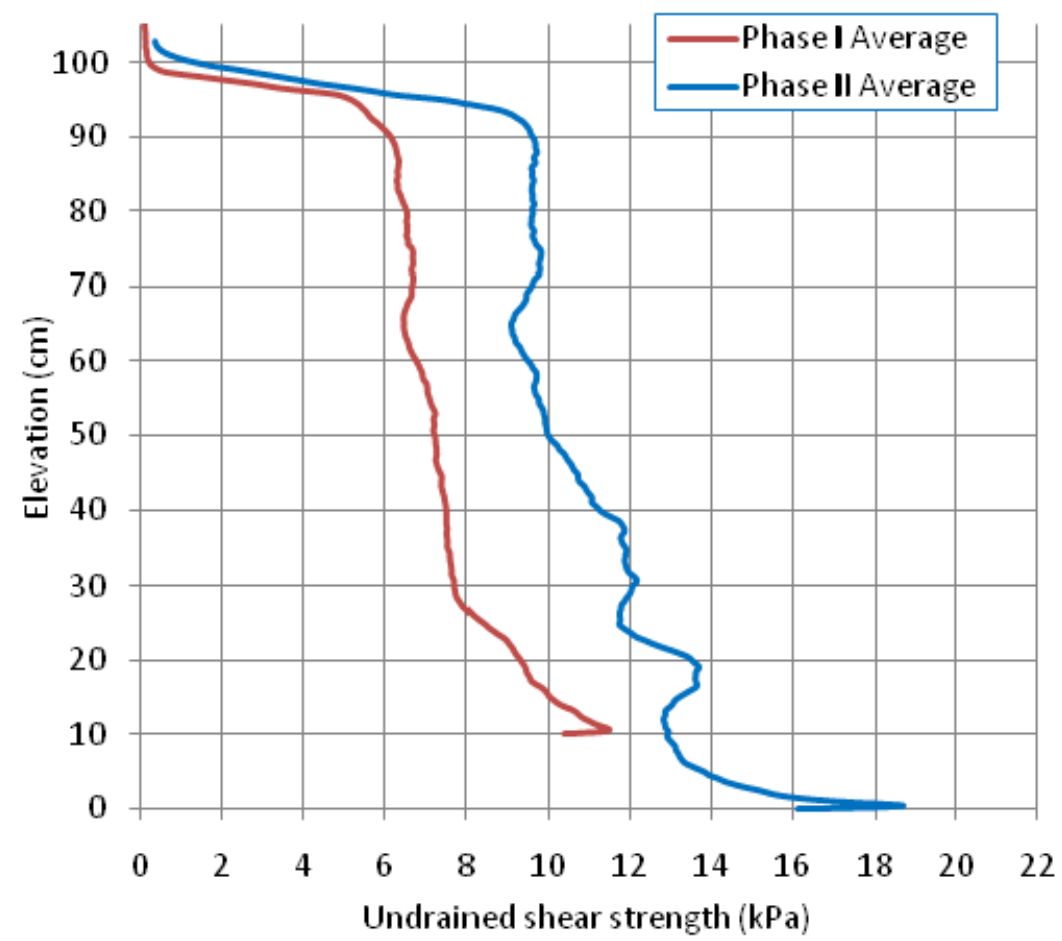

Figure 5.3 - Average undrained shear strength of the soil column using T-bar tests

This strength increase can be attributed to a combination of two factors. The first is that the overall water content of Phase II soil is lower than that of Phase I soil. The second is the position of the T-bars within the soil column. T-bars were positioned closer to the soil container sidewall to facilitate Phase II soil packing during embedment of the subway model. Seismic acceleration data recorded by the container wall accelerometer 
array during both testing phases shows possible container boundary effects. Spectral amplitudes near the container walls are higher than the middle area of the container, suggesting that soil may be stiffer near the container sidewall. This may partially explain the increase in resistance for Phase II T-bar testing. Phase II T-bar results do not provide data close to the middle of the container, which may be more characteristic of the soil surrounding the model structure.

\subsubsection{T-bar Rate Effects}

Chung et al. (2006) explains that for all types of soil penetrometers, resistance increases as penetration rate increases as long that the conditions are undrained. This is due to viscous effects as the soil flows around the penetrometer. Resistance can also increase if the penetration rate is slow enough to allow drained conditions as partial consolidation occurs. Considering the very slow rate of consolidation of the model clay and the relatively fast pullout rate of the crane, the T-bar penetration is thought to be occurring under undrained conditions and subject to viscous effects.

Biscontin and Pestana (1999) investigated the effect of shearing rate for vane shear testing in clay. The samples used for the study were obtained from Meymand's model soil column. Meymand (1998) summarized the results of the vane shear study for consideration of T-bar rate effects as shown in Figure 5.4. These results suggest that a peak strength increase of 30 to $50 \%$ can be expected for the T-bar pull out velocity of $1.29 \mathrm{~cm} / \mathrm{s}(0.51 \mathrm{in} / \mathrm{s})$ used in this study, supporting the conclusion that shearing rate effects may have contributed to overestimation of undrained shear strength during T-bar pullout testing. 
USSSI response of the structural model is not highly dependent on shear strength, so accurate characterization of undrained shear strength is not particularly important to this study. Despite this, soil resistance profiles obtained from T-bar testing are used in combination with hammer blow testing to characterize the soil stiffness, which is very important to the USSSI response of the model structure.

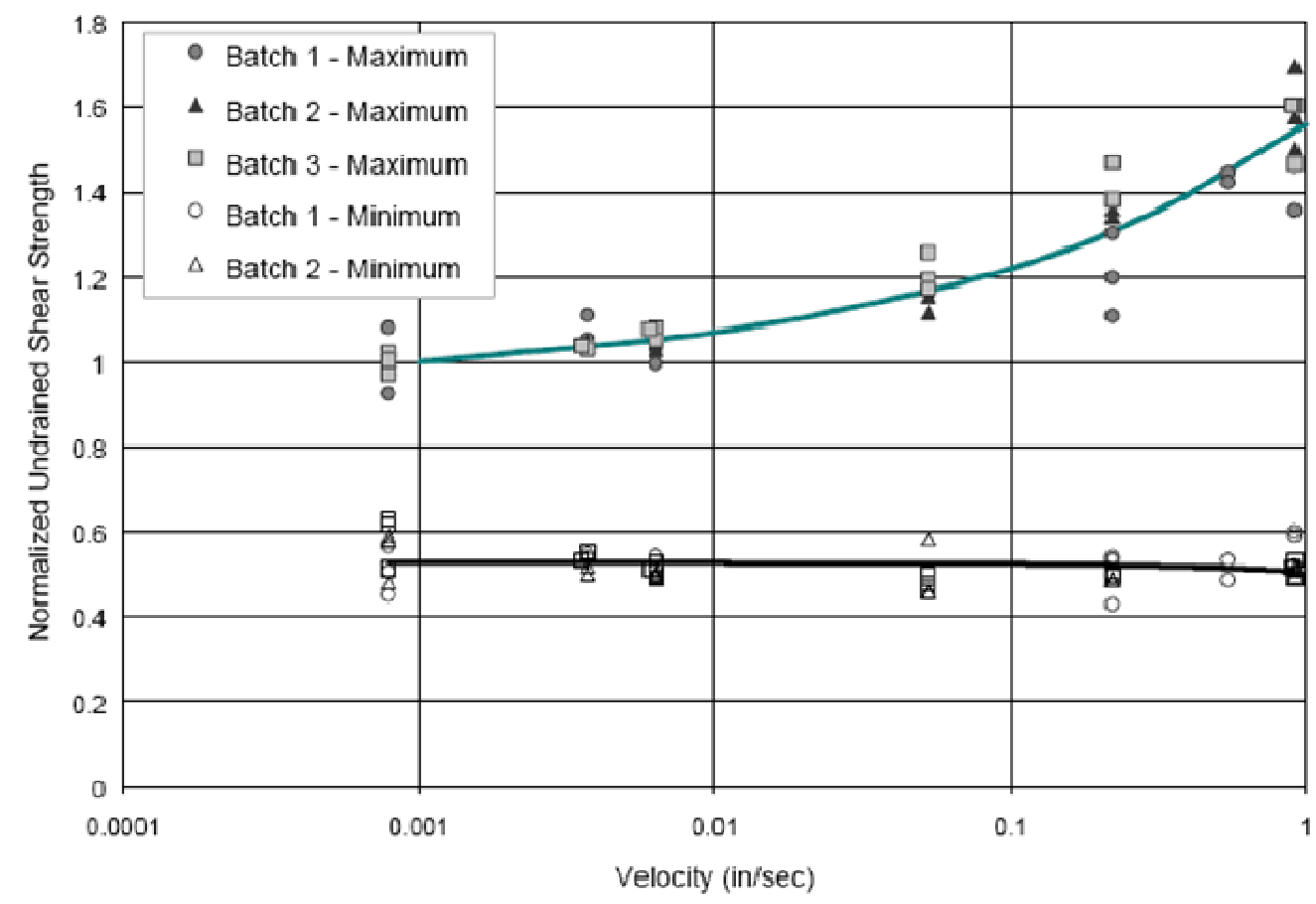

Figure 5.4 - Effect of penetrometer shearing rate on undrained shear strength measurements (from Meymand 1998)

\subsection{Hammer Blow Testing}

Hammer blow testing was conducted during Phase I testing only. This is due to the inclusion of the model subway structure in the soil. It was assumed that the inclusion of the structure would skew wave forms and wave velocity by providing contrasting material interfaces, additional wave propagation pathways, and impedance of vertically propagating seismic waves. Hammer blow testing consisted of both bottom-up and top- 
down procedures, but the top-down results are significantly more conclusive. All hammer blow tests are performed by tracking the wave propagation through the center accelerometer array in the Phase I free-field soil column. Results are presented in terms of the accelerometer positions which are shown in Figure 4.18. The bottom accelerometer (1C) is $10 \mathrm{~cm}$ above the soil column base, and the surface accelerometer (5C) is $100 \mathrm{~cm}$ above the base. Accelerometers $2 \mathrm{C}, 3 \mathrm{C}$, and $4 \mathrm{C}$ are spaced at equal intervals though the middle of the array.

\subsubsection{Bottom-up Hammer Blow Testing (series HB1)}

The Bottom up hammer blow test series HB1 consisted of striking the exterior circumference of the steel base plate with a hammer, and tracking the vertically propagating shear waves through the soil column. Figure 5.5 shows typical waveforms generated using this method, with the data expanded over a period of 0.10 seconds. The wave forms are very inconsistent and difficult to identify, which may be a result of container-soil interaction effects. These results are typical of all bottom-up hammer blow tests, using both a small framing hammer and a sledgehammer.

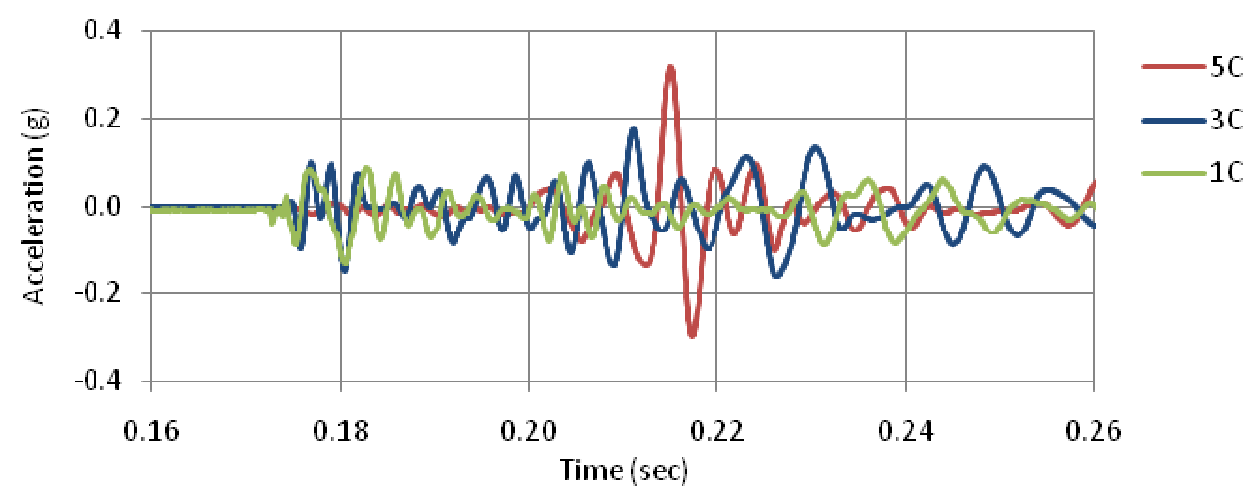

Figure 5.5 - Typical bottom-up hammer blow testing waveforms generated in the center accelerometer array (test series HB1) 


\subsubsection{Top-down Hammer Blow Testing (series HB2)}

Test series HB2 consisted of top-down hammer blow testing from the soil surface on the west side of the testing container. These tests were conducted 13 days after the bottom-up hammer blow tests in an effort to improve fidelity of the results by limiting soil-container interaction effects. As described in Chapter 4, a steel bar coupled to the soil surface was struck with a hammer, and downward vertical wave propagation was tracked though the soil. Wave forms were difficult to identify for the top two measurements positions ( $4 \mathrm{C}$ and $5 \mathrm{C}$ ), but much more clear for the bottom three positions (1C, 2C, and 3C). Figure 5.6 shows a typical hammer blow time history for the entire soil profile, where is can be seen that the wave forms near the top of the soil column are affected by high amplitude and high frequency content. Figure 5.7 shows the same results for only the bottom half of the soil column. The wave forms are much easier to track due to the dissipation of the high frequency and high amplitude content.

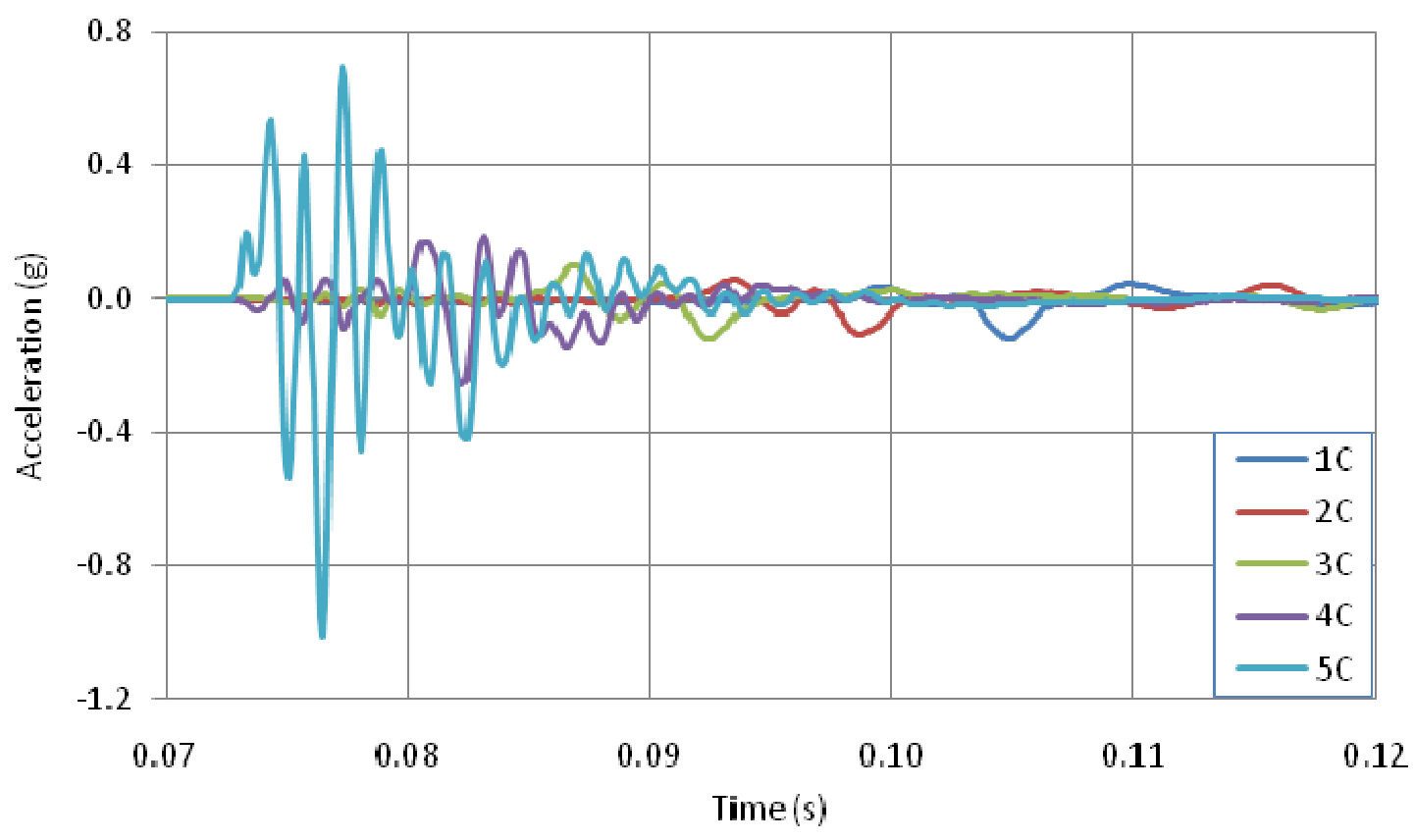

Figure 5.6 - Typical wave forms from test series HB2 for the full soil column 


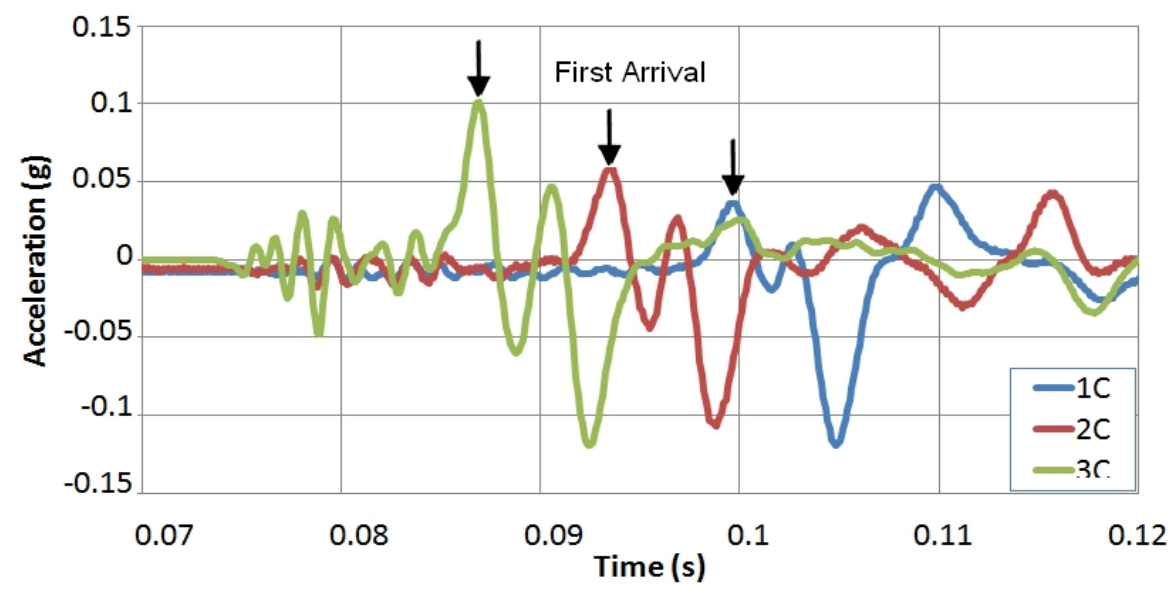

Figure 5.7 - Typical wave forms from test series HB2 for bottom half of soil profile (arrows indicate the first wave arrival used for calculating shear wave velocity)

Table 5-1 contains the results of five hammer blow tests conducted in test series HB2 for the bottom half of the soil column. The results are very consistent, showing that the lower soil layer between accelerometers $1 \mathrm{C}$ and $2 \mathrm{C}$ has a higher shear wave velocity than the layer above it between accelerometers $2 \mathrm{C}$ and $3 \mathrm{C}$. This is to be expected based on the T-bar test results presented earlier which show higher strength as depth into the soil column increases. Nevertheless, variation throughout the bottom half of the soil column is not great, indicating that overburden effects do not appreciably affect soil stiffness.

Table 5-1 Summary of top down hammer blow testing for test series HB2 in the bottom half of the soil column

\begin{tabular}{|c||c|c|c|}
\hline Accelerometer range & $1 \mathrm{C}$ to $2 \mathrm{C}$ & $2 \mathrm{C}$ to $3 \mathrm{C}$ & $1 \mathrm{C}$ to $3 \mathrm{C}$ \\
\hline Trial & $\mathrm{V}_{\mathrm{S}}(\mathrm{m} / \mathrm{s})$ & $\mathrm{V}_{\mathrm{s}}(\mathrm{m} / \mathrm{s})$ & $\mathrm{V}_{\mathrm{s}}(\mathrm{m} / \mathrm{s})$ \\
\hline$\# 1$ & 36.9 & 35.2 & 36.0 \\
$\# 2$ & 35.4 & 36.0 & 35.7 \\
$\# 3$ & 36.6 & 34.4 & 35.4 \\
$\# 4$ & 36.9 & 34.4 & 35.6 \\
$\# 5$ & 36.9 & 34.1 & 35.4 \\
\hline Average & $\mathbf{3 6 . 5}$ & $\mathbf{3 4 . 8}$ & $\mathbf{3 5 . 6}$ \\
\hline
\end{tabular}




\subsubsection{Top-down Hammer Blow Testing (series HB3)}

The top-down hammer blow test series HB3 was conducted 48 days after series HB2. The goal of this test series was to examine the effect of curing time on soil stiffness, as well as the effect of using different hammers. Testing was also conducted on both the east and west side of the testing container. A total of 24 hammer blow tests proved very consistent, resulting in shear wave velocity ranging from 38.1 to $42.9 \mathrm{~m} / \mathrm{s}$ for the bottom half of the soil profile. Hammer type and position within the testing container showed little influence on the results, except for slight increase in shear wave velocity on the east side of the container. Spatial variation is minimal compared to the effect of cure age on the soil stiffness. Average shear wave velocity in the bottom half of the free-field soil column increased from $35.6 \mathrm{~m} / \mathrm{s}$ to $40.2 \mathrm{~m} / \mathrm{s}$ from test series HB2 to HB3, an increase of about $13 \%$. This is a minor change in stiffness over a substantial period of time suggesting relatively stable soil properties over the testing period.

\subsection{Development of Shear Wave Velocity Profile}

Characterization of the full shear wave velocity profile is important for USSSI analysis, because soil stiffness is an important parameter for evaluating underground structural distortions. Shear wave velocity profiles for both Phase I and Phase II testing have been developed by considering T-bar pullout and hammer blow testing results in combination. For clay soil similar to Bay Mud, the Dickenson relationship provided in Section 3.3.1 can be used to directly estimate shear wave velocity from undrained shear strength. The relationship was empirically derived based mostly on clay samples with 
strength greater than $10 \mathrm{kPa}$, so caution should be used in applying the relationship to very soft soil such as the model clay employed in the study herein.

Applying the Dickenson relationship to the T-bar profiles overestimates shear wave velocity for the model soil by approximately 50 to $80 \%$ compared to values obtained from hammer blow testing. Accordingly, the Phase I shear wave velocity profile computed from T-bar tests was scaled to closely match testing data obtained from hammer blow test series HB2 in the bottom portion of the soil column. Shear wave velocity in the top portion of the soil column was then estimated based off of the shape of scaled T-bar results converted using the Dickenson relationship. Figure 5.8 displays the evolution of the Phase I shear wave velocity profile as a composite of top-down hammer blow data and T-bar testing data. The bottom 0.10 meters of the soil column cannot be characterized, due to a lack of data regarding the stiff clay base layer.

Meymand (1998) employed a similar technique in which shear wave velocity in the top portion of the model soil column was scaled to $32 \mathrm{~m} / \mathrm{s}$ to match results from bender element testing. Meymand's bender element results are in reasonable agreement with hammer blow tests performed in this study.

For the Phase II, the computed shear wave velocity from the Dickenson relationship was on average 20 to $25 \%$ greater than in Phase I. Thus, the profile was similarly scaled keeping this shear wave velocity increase factor in mind for scaling of the hammer blow results. There is higher uncertainty in the Phase II shear wave velocity profile due to the need to scale both hammer blow and T-bar testing results, but it provides a reasonable beginning point for calibrating equivalent linear numerical 
analyses. Figure 5.9 presents the best estimate shear wave velocity profiles for Phase I and Phase II model soil for use in numerical analyses with SHAKE and FLUSH.

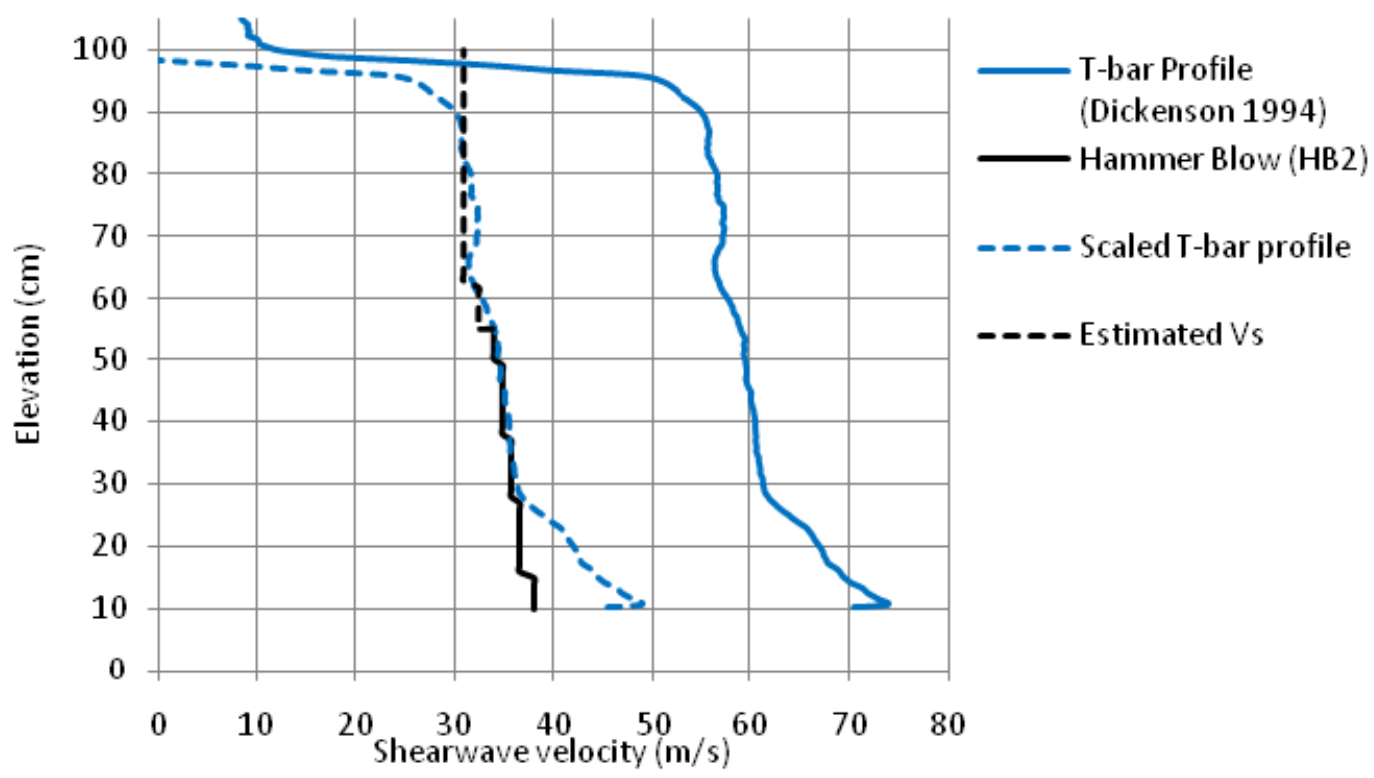

Figure 5.8 - Composite shear wave velocity profile for the Phase I soil column

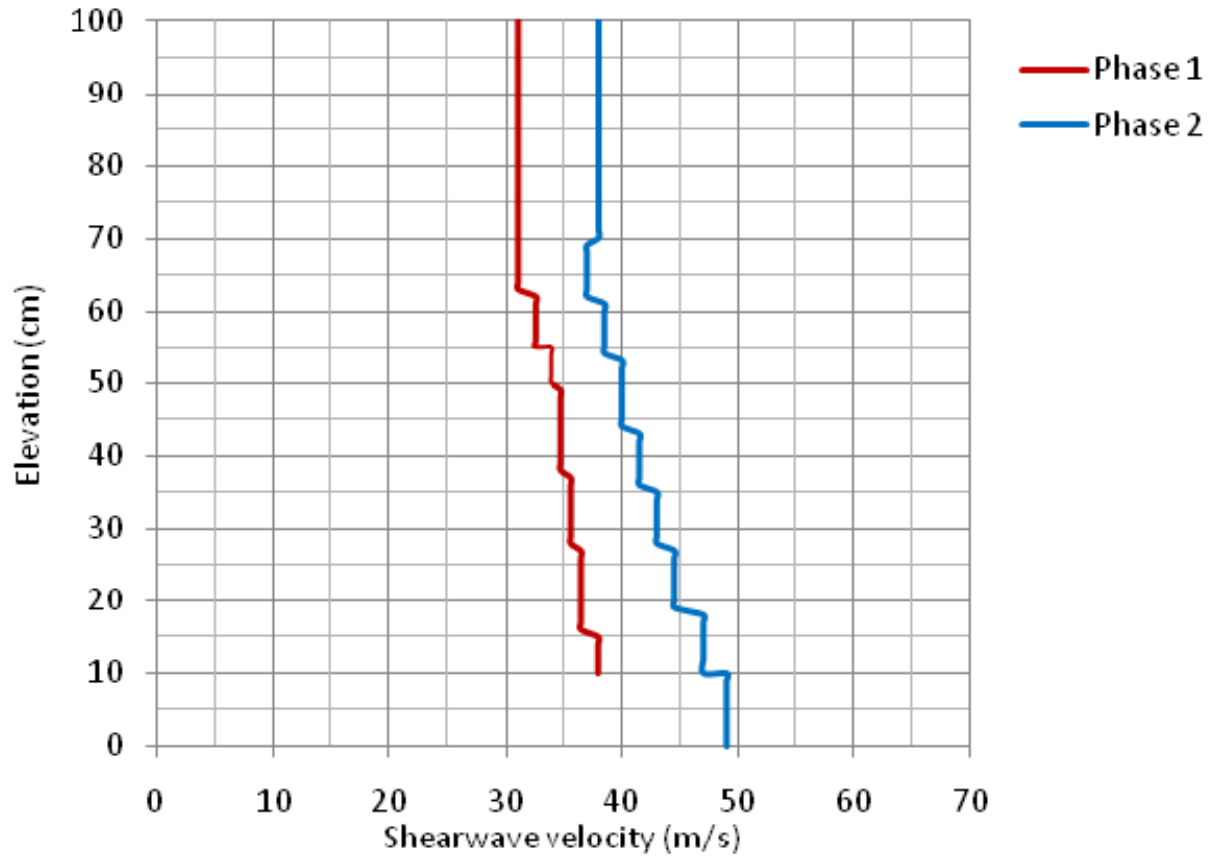

Figure 5.9 - Best estimate shear wave velocity profile for both testing phases composed from T-bar and hammer blow data 


\subsection{Shake Table Performance}

Adequate shake table performance is important for investigating historical earthquakes. Therefore, the shake table should be able to closely reproduce input motions. As previously discussed, the actual recorded table output motion is essential for running numerical simulations of model tests, especially if deviation from the desired input motion is significant. This section qualitatively investigates the ability of the table to reproduce command signals, comparing recorded acceleration, response spectra, and displacement time histories with desired input motions.

For both phases of testing, table calibration procedures were performed at shaking intensities less than those of the full test motions, consistently resulting in full test motions with ground accelerations greater than the desired input motion. The shake table system scales command signals linearly. Therefore, any nonlinear response of the soil and container is not accounted for, leading to amplification of the table output at full testing levels. For most of the experiments, the output Peak Ground Acceleration (PGA) is greater than the input. The effect of increased table output accelerations can best be observed by comparing response spectra of the recorded table motion and the desired input motion. Response spectra for the Phase I free field Joshua Tree motions are presented in Figure 5.10, showing high output spectral acceleration for the table compared to the desired input motions. The most significant variation occurs in the high frequency regions down to about $10 \mathrm{~Hz}$. The spectral match at lower frequencies is much better for all of the free-field testing motions. This longer period match is encouraging because the prominent site period of the free-field soil column is greater than 0.1 seconds, as will be shown in the following sections. 

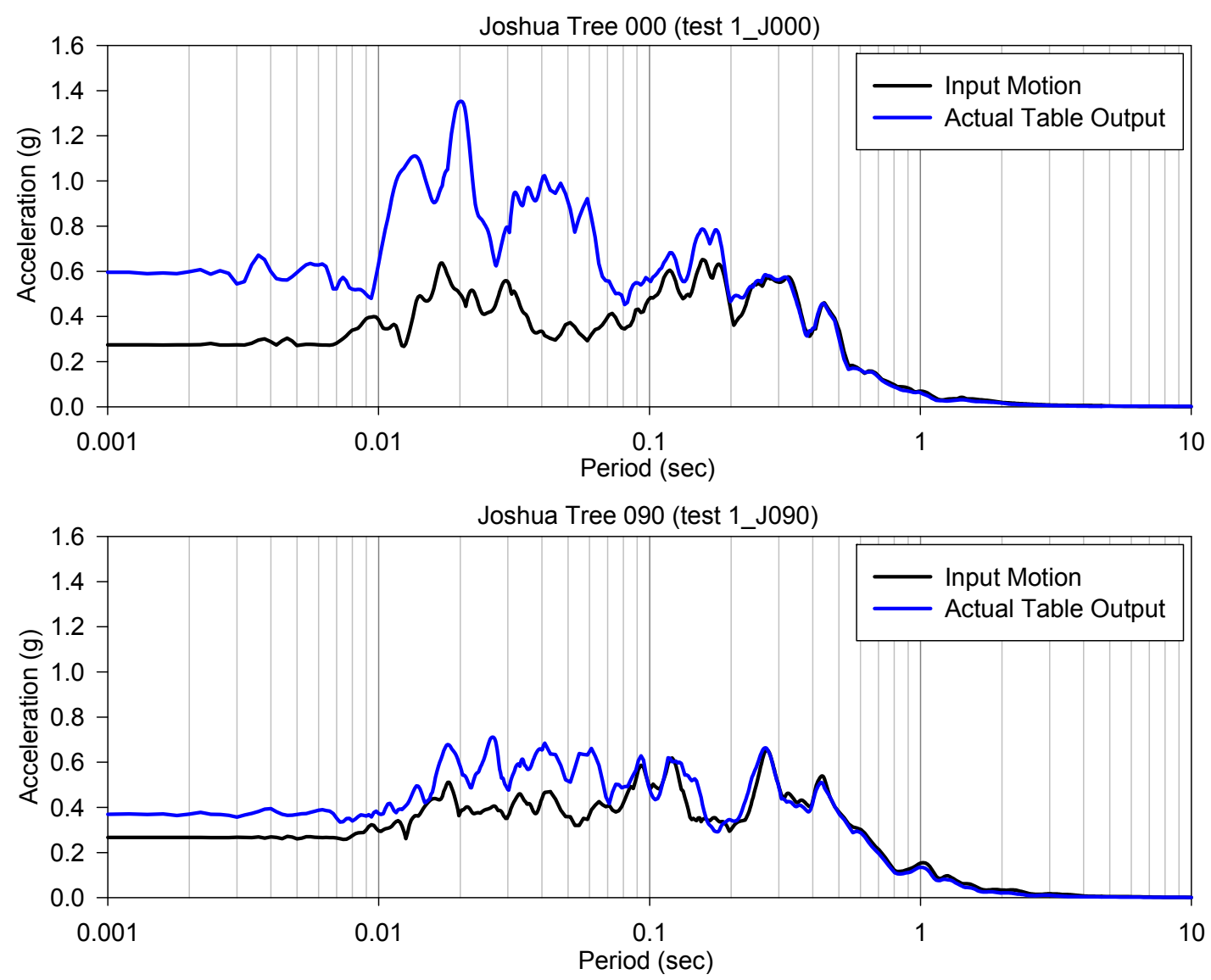

Figure 5.10 - 5\% damped response spectra comparing desired input motion and actual recorded output motion for both Phase I free-field Joshua Tree motions

Comparing absolute displacement time histories of the input and output motions can also provide insight on table performance. Variation between the input and output can be attributed to inadequate table response as well as error resulting from the mathematical transformations required to obtain displacement from acceleration. Also, all tests were conducted using the "DC Remove" filter in the Dactron shaker control software, which automatically makes baseline adjustments to the input motions. In turn, this filter may have also altered acceleration output compared to that of the desired input motion. Despite these issues, the table did manage to reproduce approximate displacement patterns. The TCU075 North motion provides excellent displacement 
matching between the input motion and output recording, as shown in Figure 5.11. Other motions resulted in less adequate displacement matching, partly resulting from the effect of the DC Remove filter, as shown in the displacement recording for the El Centro 180 motion in test 2_E180.3 (Figure 5.12).

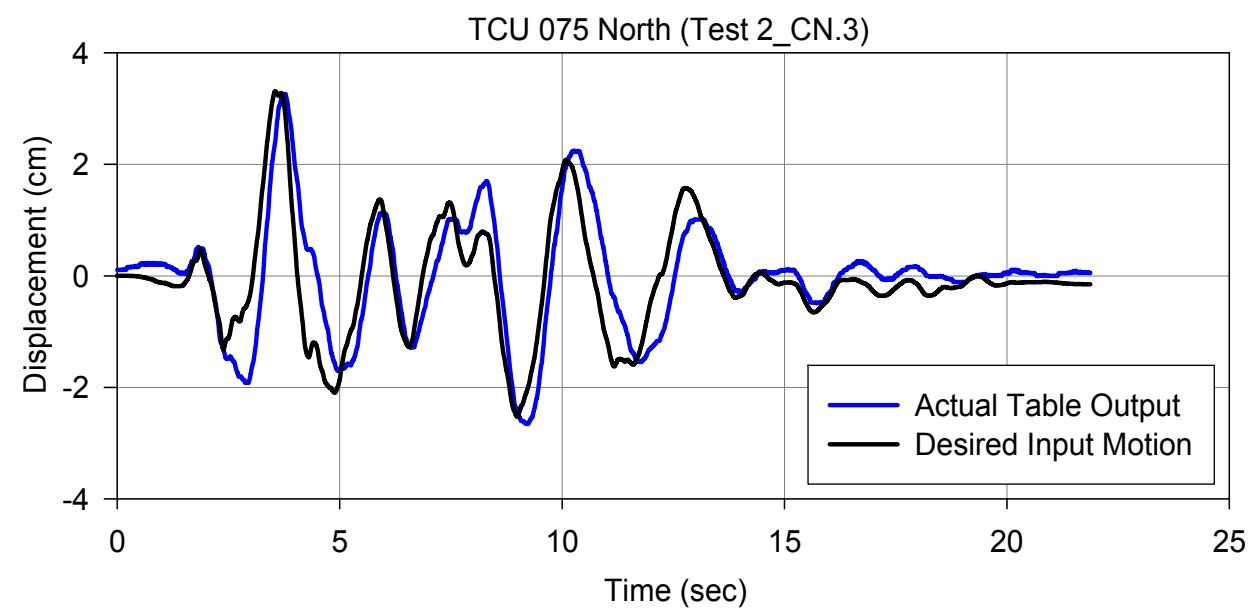

Figure 5.11 - Comparison of input and output table displacement time histories for TCU075 North (Test 2_CN.3)

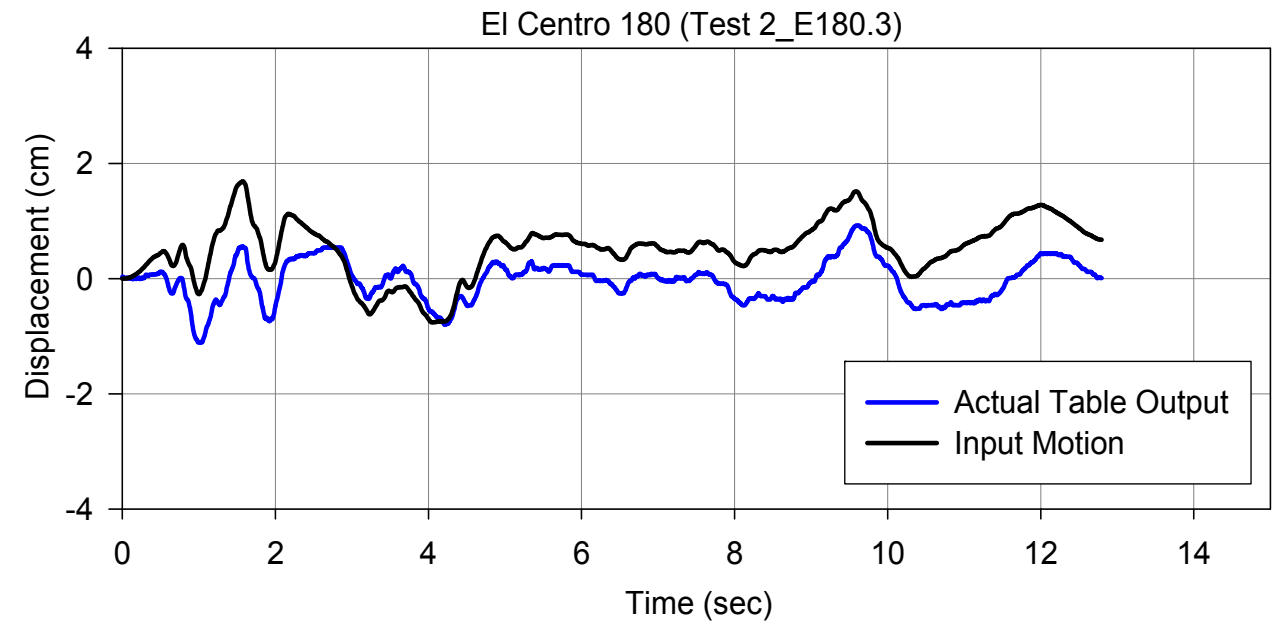

Figure 5.12 - Comparison of input and output table displacement time histories for El Centro 180 (Test 2_E180.3)

Overall, the table showed sufficient performance in matching both acceleration and displacement time histories. Some of the short period content is an expected result of 
noise typical of uncorrected motions. This noise has negligible effect on the predominant site period and numerical simulations. Some of the table response error can be partly attributed to human error in running the shake table, which may alleviate as experience by future Cal Poly researchers is gained. Two suggestions for improving shake table performance are: (1) avoid using filters by inputting motions that are initially baseline adjusted, and (2) use command signals created at similar shaking intensity levels as the desired test motions.

\subsection{Site Amplification Effects}

It is well understood that soil deposits can amplify seismic motions at the soil surface relative to bedrock motion. During the 1989 Loma Prieta earthquake, site amplification of peak acceleration at soft soil sites was observed to be as much as four times that of nearby rock recordings (Idriss 1990). At very high levels of shaking, amplitude may be comparable or less than that of rock sites due to nonlinear effects in soil response. The shaking magnitudes induced in this study are such that highly nonlinear effects such as soil shearing are not pronounced, resulting in the soft model clay exhibiting substantial amplification effects. This site amplification can be easily tracked though vertical accelerometer arrays and observed as acceleration time histories and response spectra. Figure 5.13 shows the recorded accelerations and corresponding $5 \%$ damped response spectra for the free-field Joshua Tree 000 motion in the center vertical accelerometer array. Amplification effects through the soil column are typical of all tests during both testing phases. Similar plots for the other Phase I shake table tests are provided in Appendix B. 

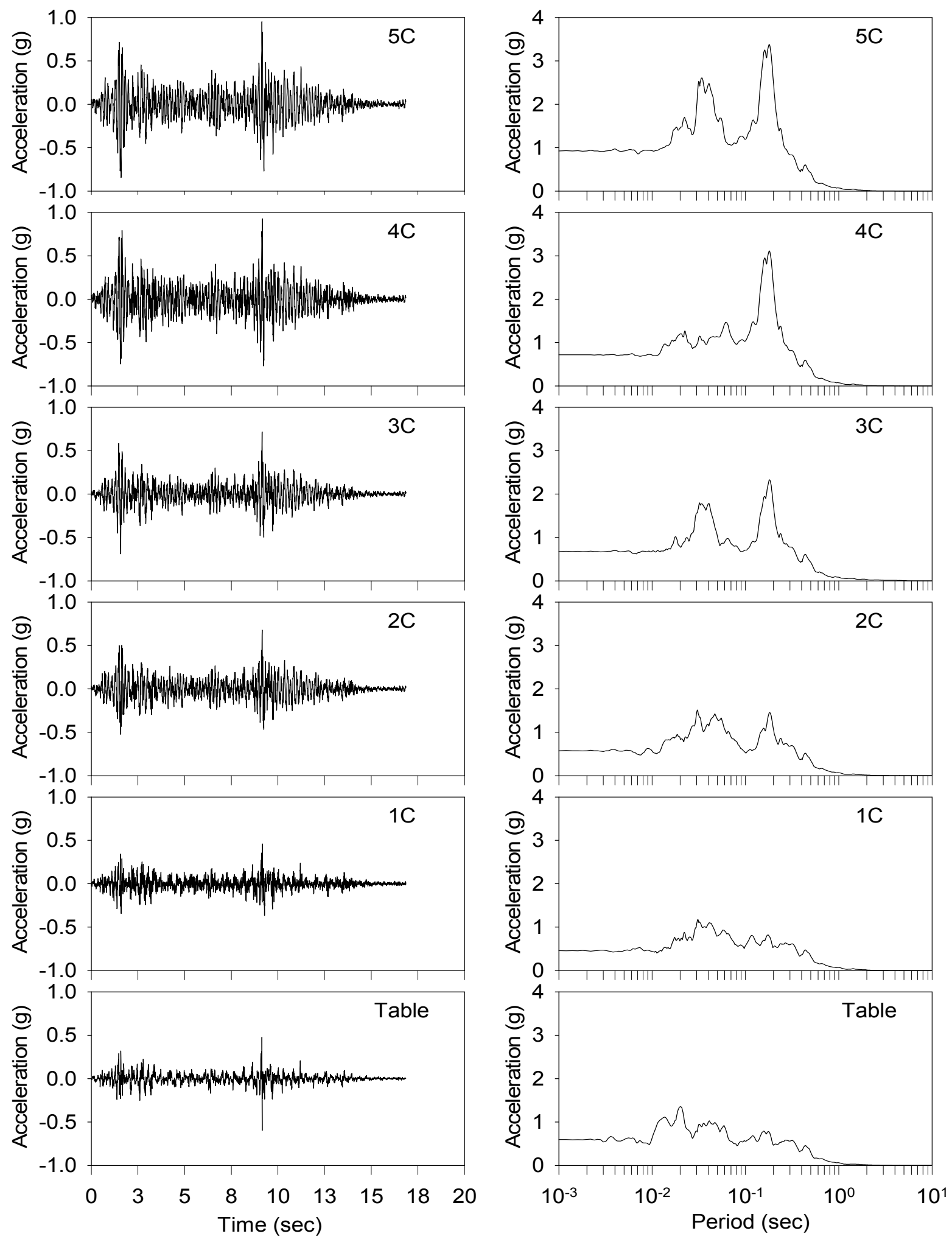

Figure 5.13 - Acceleration and 5\% damped response spectra recorded at the center accelerometer array for the free-field Joshua Tree 000 motion (test $1 \_$J000) 
Soft soil sites also tend to shift frequency content to lower frequencies than those characteristic of stiffer soil or rock sites. This is apparent from testing results, as the primary resonant period shifts from shorter periods at the table to longer periods at the soil surface, with the transition point being somewhere in the middle of the soil column. According to Boore and Bommer (2005), signal noise can adversely affect the motion parameters without the application of a high frequency cutoff filter. This may explain some of the high frequency variability, but there does seem to be a common resonant peak at short periods across all of the free-field motions. The short period peaks may be characteristic of the soil column, table interaction effects, or the natural frequency of the embedded accelerometer foundations. The predominant longer period modes are of primary interest in this study since they are the most characteristic of actual free-field response.

\subsection{Testing Container Performance}

The ability of the test container to approximate one-dimensional (1D) site response is important to the validity of seismic SSI testing for any type of structure. For the 1D site response assumption to be valid, all points within any horizontal plane should be subjected to very similar ground motions. Meymand (1998) examined this by comparing response spectra through multiple vertical accelerometer arrays within the test container and found that the coherence of the motions within any particular horizontal plane was excellent. The following sections provide discussions relating to the ability of the container to provide consistent one-dimensional response in terms of possible boundary effects, motion coherence throughout the soil column, and test repeatability. 


\subsubsection{Container Boundary Effects}

Meymand's SSPSI study did not look into the effect of the container sidewall on ground motion, because no accelerometer arrays were placed within 0.3 meters of the rubber membrane. For Phase I free-field experiments in this study, a single array of 3 accelerometers was placed within $15 \mathrm{~cm}$ of the rubber membrane along the shaking axis. The wall accelerometer array revealed higher spectral acceleration near the container sidewall at primary resonant periods, indicating that soil-container interaction may be altering soil response near the boundary relative to free-field response. This is an undesirable effect because it limits the effective diameter of the free-field soil column.

Qualitatively observing acceleration time histories shows that wave amplitude and waveforms are similar between the wall and the center accelerometers at similar embedment depths. Additionally, there is no distinct phase shift between the accelerations at any given depth. The only consistent difference between the boundary soil and the more interior soil is the slight increase in spectral response near the container wall. Figure 5.14 shows the ratio of maximum 5\% damped spectral acceleration near the container wall relative to the center of the soil column for all six free field motions.

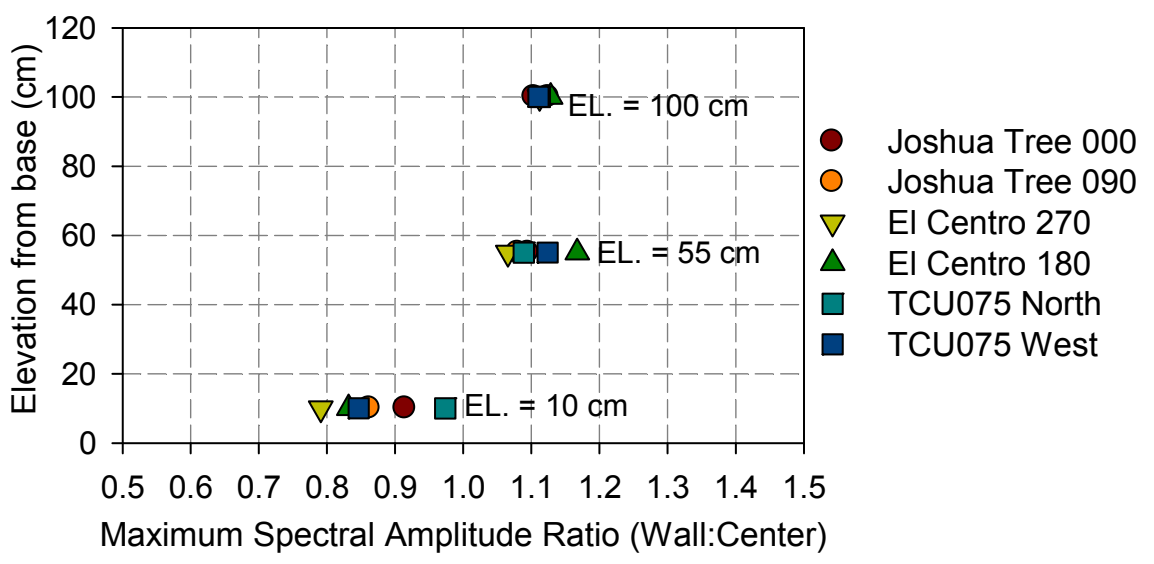

Figure 5.14 - Ratio of maximum 5\% damped spectral acceleration of the wall array relative to the center array 
Near the surface, the maximum spectral acceleration at the boundary is about $10 \%$ greater than at the center of the soil column. This average increase is similar near the middle depth of the soil, but with more spread between tests, possibly being a function of the period shifting. Near the soil column base, maximum spectral amplitude at the boundary is lower, but this occurs at short periods where spectral response is more randomly affected by noise and other undesirable signal sources. It is important to note that only the amplitude is affected by boundary effects, and there is no significant shift in predominant periods at the boundary compared to the center. The overall contribution of container boundary effects appears to be minor, but it is significant enough to warrant avoiding placing model structures close to the container wall, especially along the primary shaking axis.

\subsubsection{Motion Coherence}

Since the purpose of this project is to study the response of a buried structure subjected to seismic loading, 1D soil column response in the subsurface is critical. Figure 5.15 shows the spectral response of four Phase I accelerometers placed at an elevation of $55 \mathrm{~cm}$ within the soil column for both azimuths of the El Centro motion. Peak ground acceleration values match very closely for the different positions within the horizontal plane except for the offset position. At the site period, the amplitude of the spectral response is very similar for the center (3C) and middle (3M) positions, with an increase in amplitude for the wall position ( $3 \mathrm{~W})$ and a decrease for the offset position (3O). The lower amplitude at the offset position may be attributed to two-dimensional 
effects of the test container, but it is more likely that the accelerometer was misaligned resulting in decreased response.
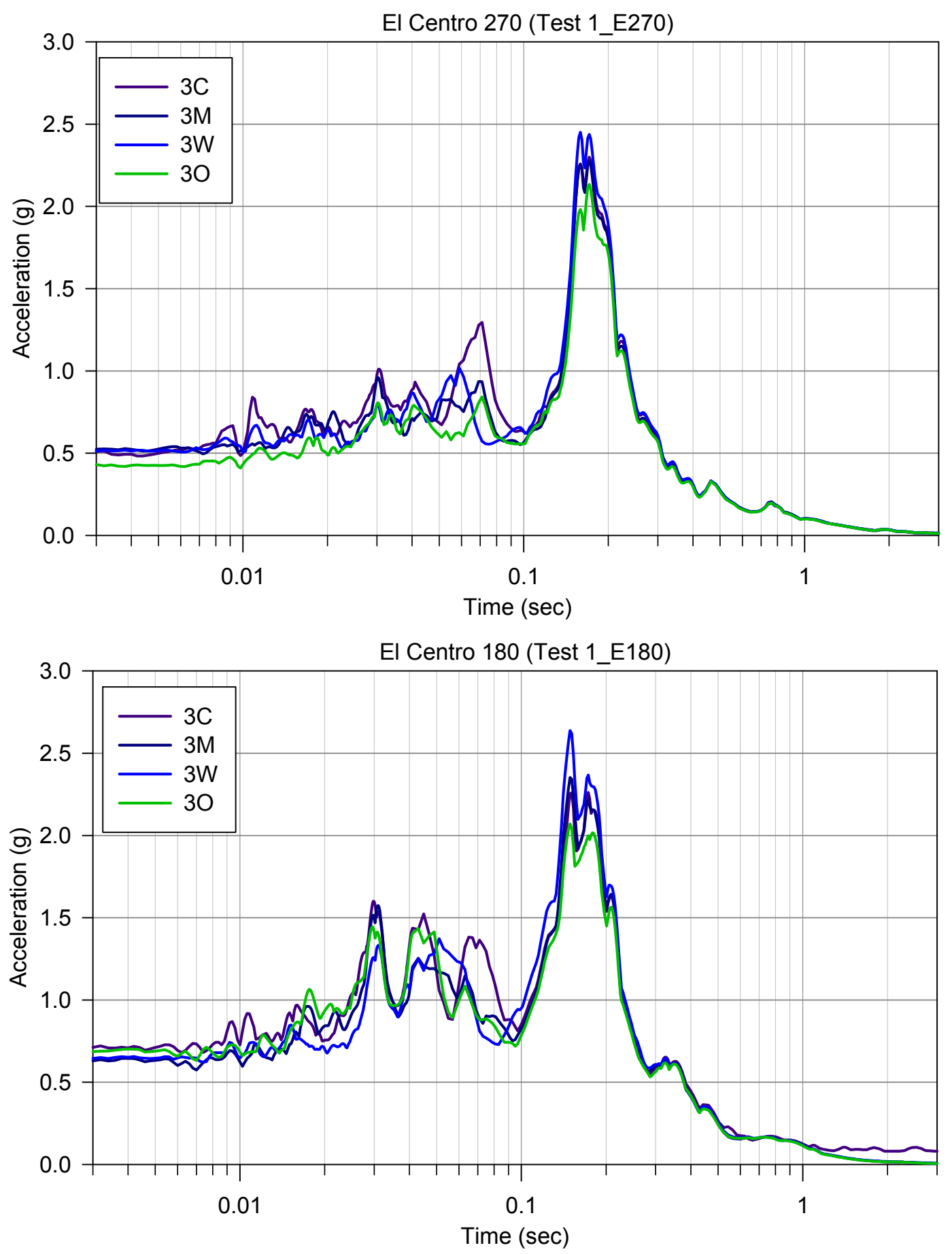

Figure 5.15 - 5\% damped response spectra for Phase I El Centro testing at an elevation of $55 \mathrm{~cm}$ in the soil column 
Misalignment of the Phase I offset accelerometer is evidenced by the lack of significantly reduced amplitude at the offset position during Phase II testing. At short periods, the coherence of the motions breaks down and becomes much more random. Overall, the shape and magnitude of the spectral response shows adequate motion coherence at the subway model embedment depth. These patterns are typical of all tests performed during Phase I testing.

Phase II testing resulted in better motion coherence within horizontal planes than Phase I testing. The offset position at $55 \mathrm{~cm}$ elevation (3O) did not produce a significantly reduced signal when compared to the middle position (3M). The wall array behaved similar in Phase II in that it produced increased spectral amplitudes compared to the other positions within individual horizontal planes, but the effect was not as pronounced as in the free-field testing. This may indicate that the soil around the structure experienced a stiffening effect similar to the boundary soil. Another possible explanation is that the since the Phase II soil was initially stiffer, the one-dimensional performance of the test container was improved. In either case, the Phase II container performed well in mimicking one-dimensional response. Figure 5.16 shows the coherence of the TCU075 North motion (test 2_CN.3) as a plot of 5\% damped response spectra for all accelerometers placed within individual horizontal planes.

The higher frequency content in Phase II testing still suffers from inconsistency as in Phase I testing, but the effect not quite as pronounced. The site period is reduced from approximately 0.15 to 0.18 seconds for free-field testing to approximately 0.10 to 0.12 seconds across all Phase II tests. This is likely due to the initially stiffer soil and may be partly attributed to the attached soil block effect provided by interaction with the 
embedded structure, as presented in Huo et al. (2005). Summary tables for Phase I and Phase II testing showing predominant periods, peak ground accelerations (PGA), and maximum spectral amplitudes are provided at the end of this chapter.

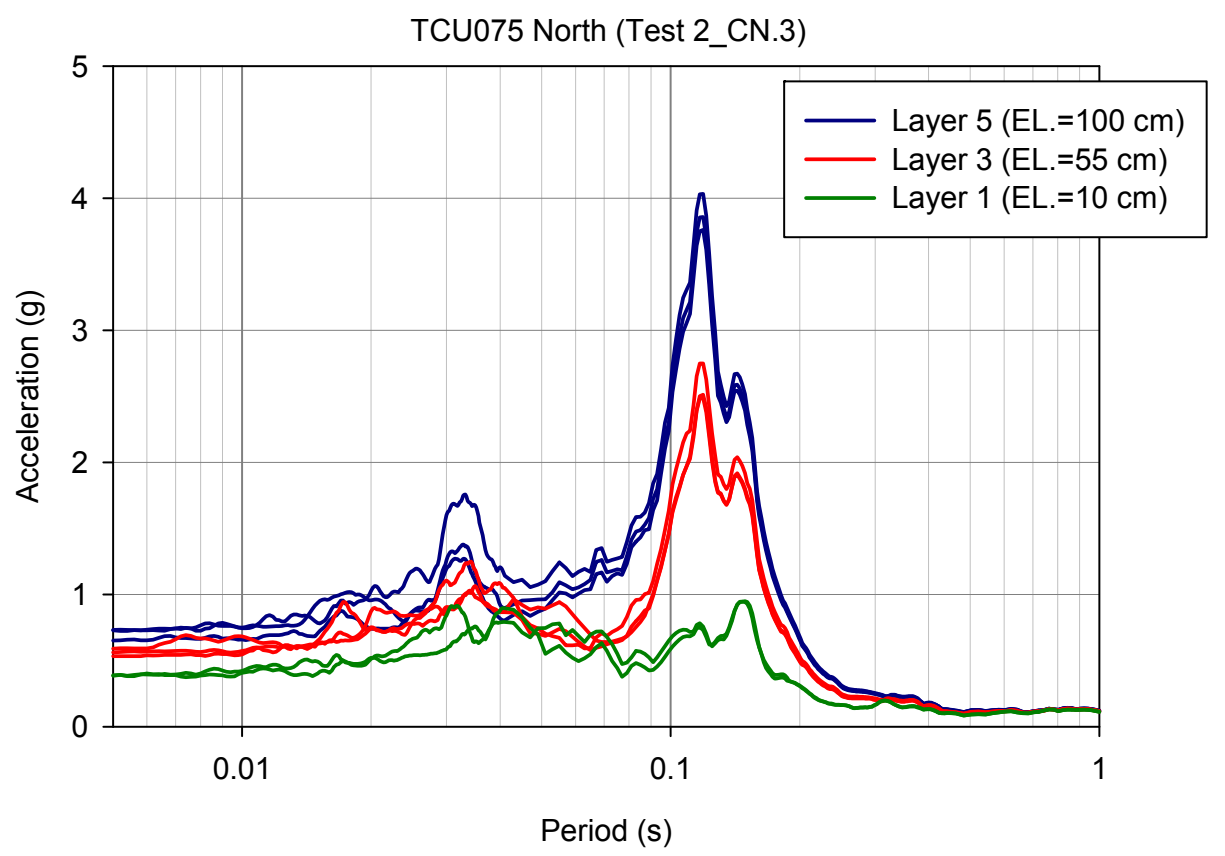

Figure 5.16 - 5\% damped response spectra for the Phase II TCU075N motion at elevations of $10 \mathrm{~cm}, 55 \mathrm{~cm}$, and $100 \mathrm{~cm}$ in the soil column

\subsubsection{Test Repeatability}

The ability to duplicate tests is important if the goal of a testing program is to explore the effect of changing certain variables while holding all other factors constant. Both free-field and USSSI experiments in this test program aim to hold all variables constant except for the input motion, in which six total motions were tested. During Phase II testing, the Chi Chi earthquake TCU075 tests were duplicated at the full 100\% testing intensity to evaluate the test repeatability of the container and scale model. The repeat tests were performed four days after the initial tests. Results show excellent test repeatability in terms of acceleration, absolute displacements, and structural distortions. 
Additionally, many other motions were tested in between the repeated tests, indicating that there was minimal site degradation. Figure 5.17 presents overlaid response spectra from the repeated tests at the table and at the top of the soil column (accelerometer 5C). The TCU075 West motion shows significant variation at short periods for the table recording, but this variation quickly attenuates through the soil column.
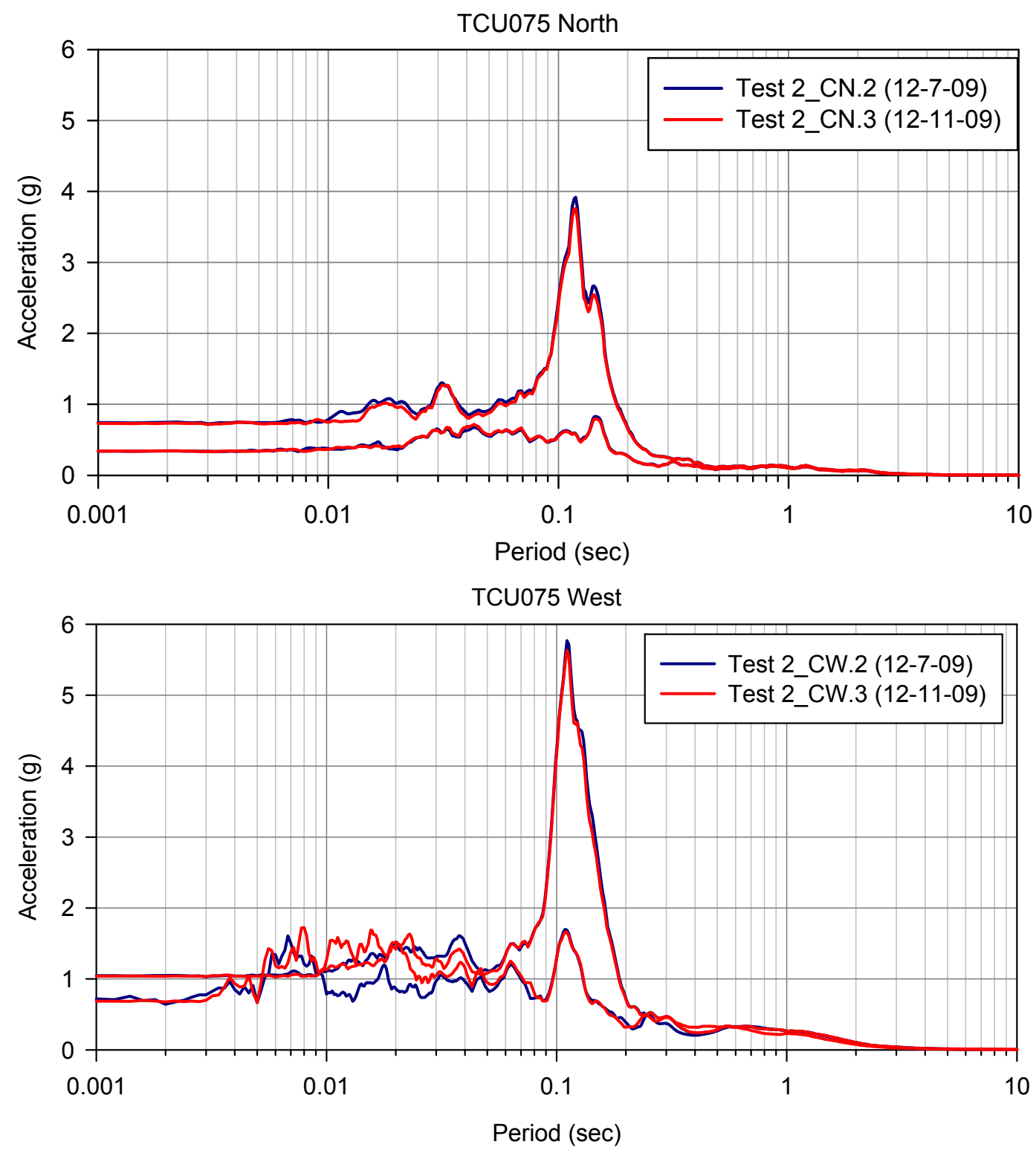

Figure 5.17 - Test repeatability illustrated by $5 \%$ damped response spectra at the table and soil column surface (position 5C) for both Phase II TCU075 motions 


\subsection{Structural Racking}

The primary purpose of Phase II shake table testing was to evaluate seismic transverse racking on a stiff rectangular tunnel cross-section embedded in soft clay. This section presents the results of the USSSI testing in the form of racking distortion time histories within the structure. Two LVDT's were connected to the stiff mounting arm to measure simultaneous distortions near the top of each sidewall relative to the floor of the structure (refer to Figure 4.20). The main reason for measuring opposite walls was redundancy in case of instrument failure and to confirm the stability of instrument mounting. A secondary benefit was to confirm that the structural distortions are equal at each sidewall. Examining the distortion recording shows equal and opposite distortions between the two instruments with time, consistent with the opposite measuring directions of the LVDT's. This indicates that the horizontal distortions at each side wall are identical, confirming that the deformational shape of the structure is characteristic of simple shear. A short portion of the racking results from test 2_J000.2 are shown in

Figure 5.18 to illustrate the equal sidewall distortions typical of all Phase II tests.

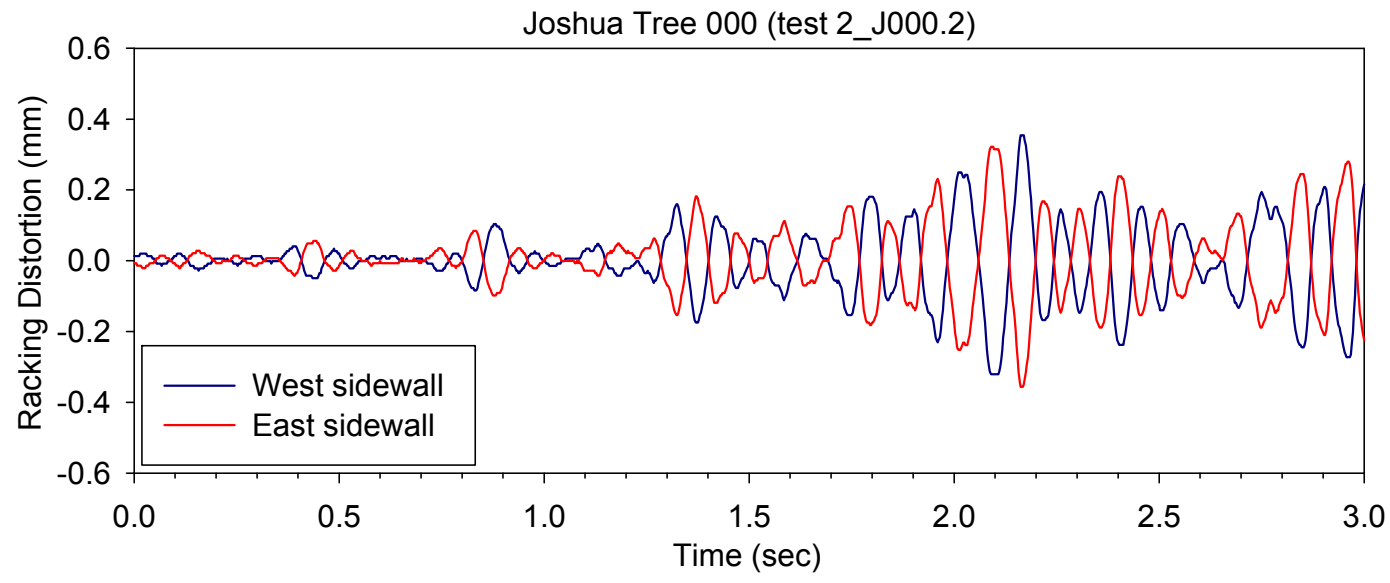

Figure 5.18 - First 3 seconds of the Joshua Tree 000 racking time history illustrating equal horizontal distortion recorded at each structural sidewall. 
Maximum racking distortion was the primary value of interest for the USSSI testing. From the primary test motions, the largest maximum racking distortions of 0.80 mm occurred as a result of the TCU075 West tests (2_CW.2 and 2_CW.3). The lowest racking distortions observed for a full intensity test were the result of the El Centro 270 test (2_E270.3), which had a maximum racking distortion of $0.53 \mathrm{~mm}$. It is important to note that the recorded relative distortion is not measured at the extreme top and bottom of the structural section. It is measured from just below the underside of the ceiling slab relative to the top of the floor slab. Distortion time histories are shown for tests 2 CW.2 and 2_E270.2 in Figure 5.19 and Figure 5.20 respectively.

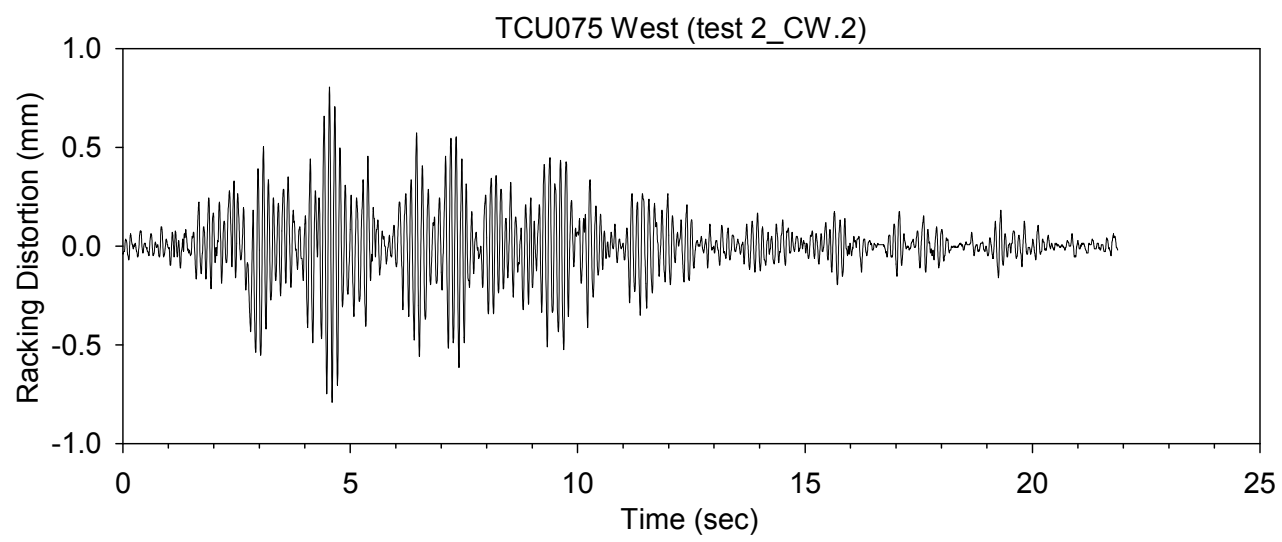

Figure 5.19 - Racking distortions for the TCU075 motion (test 2_CW.2)

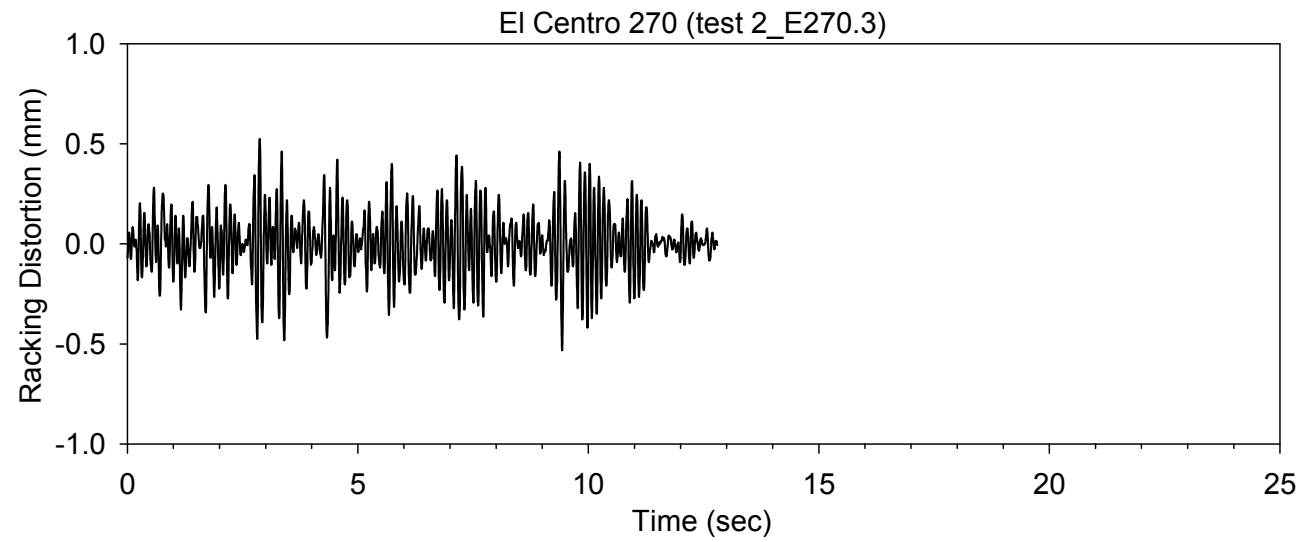

Figure 5.20 - Racking distortions for the El Centro 270 motion (test 2_E270.3) 
The distortion recordings show a very similar shape to the corresponding acceleration time histories recorded at the ceiling slab of the buried structure. This qualitatively implies that the magnitude of structural distortion is related to the applied acceleration. Figure 5.21 overlays ceiling acceleration with racking distortions recorded at the east sidewall to illustrate the shape similarity of the time histories for test 2 CN.2.

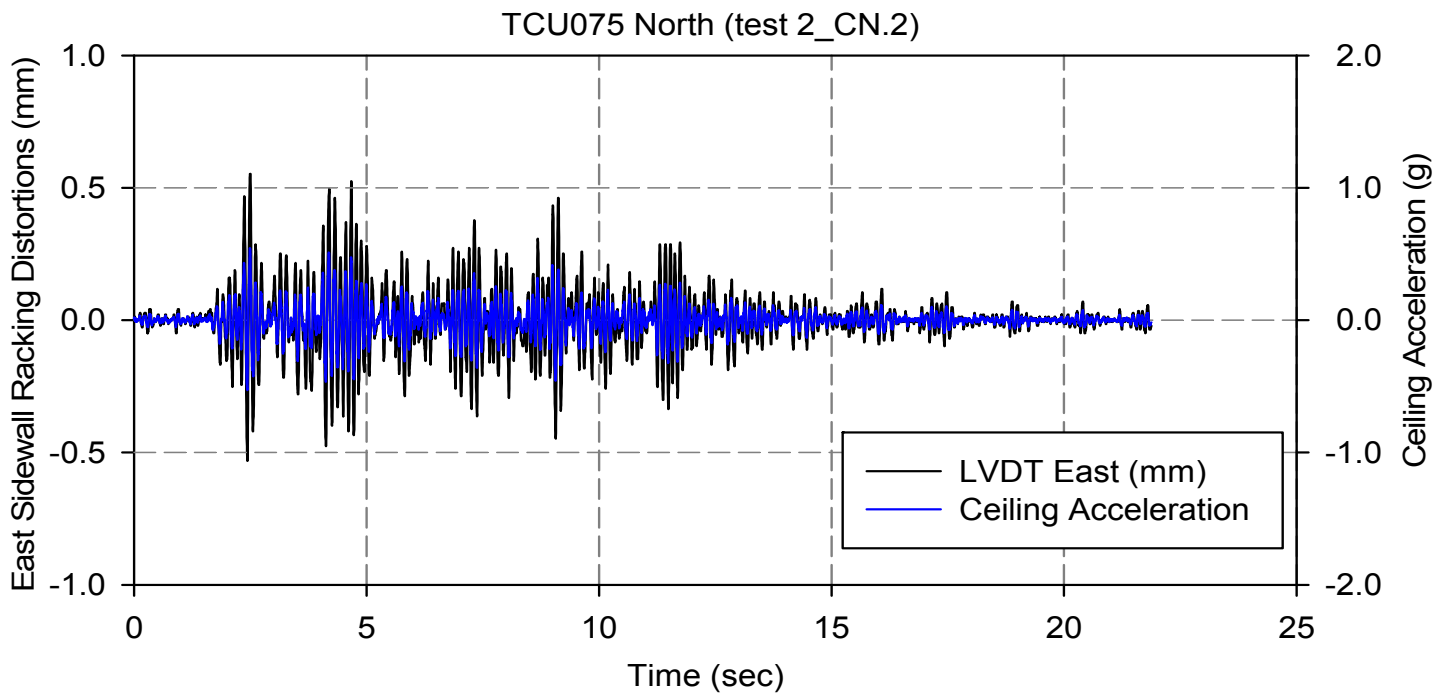

Figure 5.21 - Ceiling slab acceleration time history overlaid with the LVDT East distortion recording for the TCU075 North motion (test 2_CN.2)

Plotting the maximum differential acceleration between the ceiling slab and floor slab (herein called "differential racking acceleration") against the maximum recorded racking distortion illustrates a clear linear relationship. This makes sense in terms of linear elastic structural response, since structural distortions are proportional to applied force which is proportional to acceleration. Figure 5.22 plots distortion against differential racking acceleration for all seventeen Phase II shake table tests, showing that the relationship seems to be independent of the motion. This is consistent with conclusions by J.J. Wang (1993) who found that motion type had little influence on 
racking response. The data presented here only applies to the specific structural model tested in this study, and further testing would be necessary to make similar conclusions regarding other structural configurations.

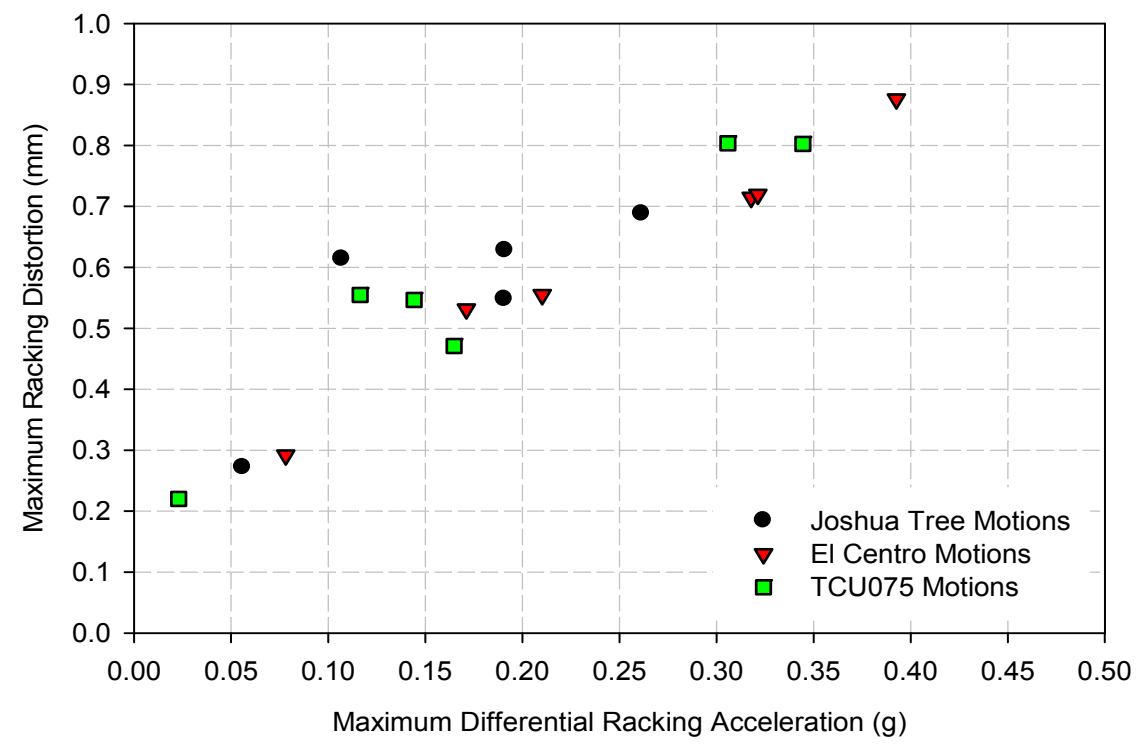

Figure 5.22 - Plot showing the relationship between maximum differential racking acceleration and maximum racking distortion

The acceleration at the top of the stiff LVDT arm was consistently greater than the acceleration recorded on the floor slab. There are at least three possible reasons for this. First, the entire structure could be experiencing a rocking motion. Rocking motions are consistent with Penzien's (2000) assertion that rigid body rotation accompanies transverse racking of rectangular structures. Second, curvilinear distortion of the floor slab due to the stiff structural corners may result in rocking motions of the stiff arm relative to the rest of the structure. Third, the LVDT arm may be allowing some distortion due to shear and bending throughout the arm. Rocking of the entire structure would not affect relative distortion measurements, but rocking or distortion of the arm only may be a source of measurement error. 
Increased acceleration at the top of the LVDT arm relative to the floor is probably a result of a combination of all three of these factors, with rigid body rotation being the most pronounced. Meymand (1998) reported that bending of the soil column does occur with this flexible wall system, but it does not have a significant detrimental effect on the container performance. Soil column bending could also contribute to rocking of the structure, which would not cause significant error in distortion measurements. Further research would be needed to characterize the magnitude of rocking effects as a result of soil column bending and rigid body rotation. Based on a very conservative cantilever analysis, distortion of the arm is considered negligible due to its high stiffness relative to the structure. Deformation of the stiff arm orthogonal to the shaking direction is negligible based on the relatively low acceleration response in that direction. Figure 5.23 plots response spectra for test 2_CW.2 for all acceleration recordings within the structure showing higher amplitude response at the ceiling slab and LVDT arm relative to the floor slab.

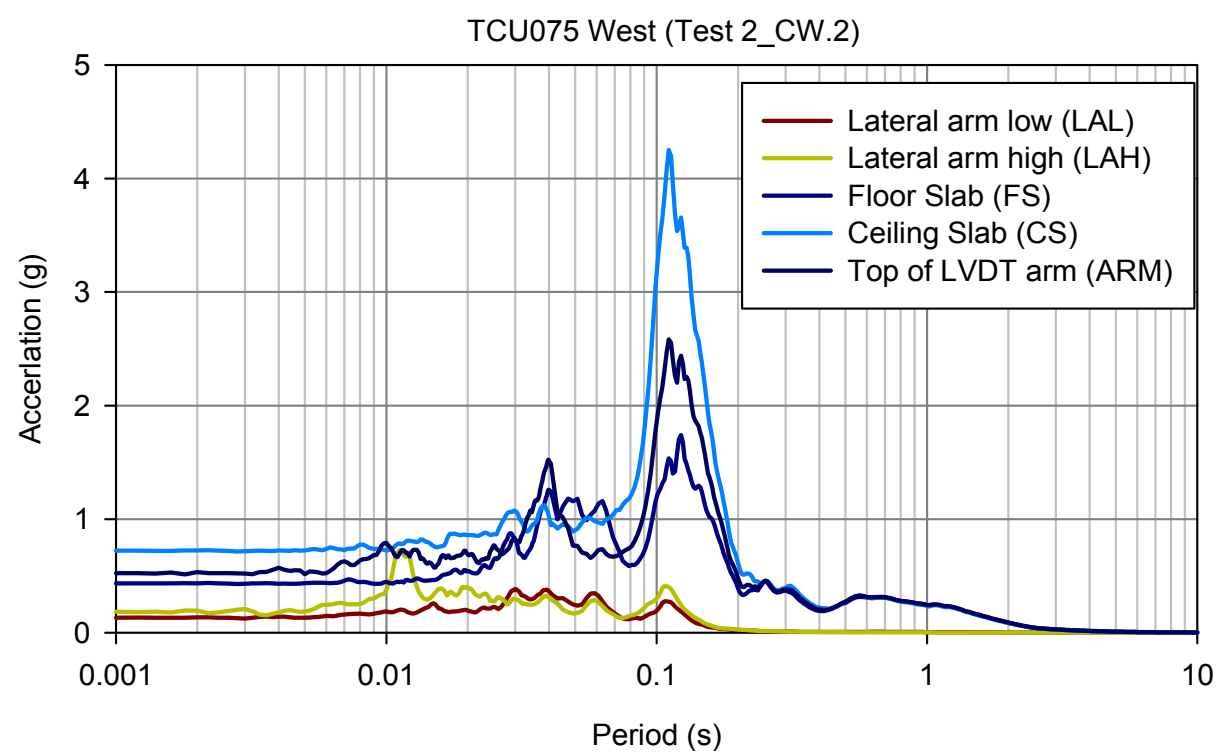

Figure $5.23-5 \%$ damped response spectra within model structure (test 2_CW.2) 


\subsection{Phase I Free-Field Results Summary}

Contained in this section is a summary table of results from all testing during Phase I containing peak ground accelerations and maximum 5\% damped spectral amplitude near the bottom of the soil column (accelerometer 1C) and at the soil surface (accelerometer 5C). Also shown is the predominant scale model site period corresponding to the maximum spectral amplitude at the soil surface. The site period is relatively consistent within a range of 0.149 seconds for the El Centro 180 motion to 0.179 seconds for the Joshua Tree 000 motion. It is important to note that the site period listed here is not necessarily characteristic of the shape of the response spectra. For example, the Joshua Tree 000 motion contains a slightly lower amplitude peak at a period of 0.159 seconds within the predominant amplitude spike. Appendix B contains acceleration time histories and 5\% damped response spectra recorded at the center accelerometer array for all of the Phase I tests.

Table 5-2 Summary table of Phase I testing results at the bottom (1C) and top (5C) of the free-field soil column

\begin{tabular}{|c|c|c|c|c|c|c|}
\hline \multirow{2}{*}{ Test ID } & \multirow{2}{*}{ Test Motion } & \multicolumn{2}{|c|}{$\begin{array}{c}\text { Peak Accelerations } \\
(\mathrm{g})\end{array}$} & \multicolumn{2}{c|}{$\begin{array}{c}5 \% \text { Damped } \\
\text { Spectral } \\
\text { Amplitude (g) }\end{array}$} & $\begin{array}{c}\text { Site } \\
\text { Period } \\
\text { (seconds) }\end{array}$ \\
\cline { 3 - 6 } & & 1C & 5C & 1C & 5C & \\
\hline 1_J000 & Joshua Tree 000 & 0.459 & 0.927 & 1.17 & 3.38 & 0.179 \\
\hline 1_J090 & Joshua Tree 090 & 0.338 & 0.668 & 0.902 & 2.74 & 0.153 \\
\hline 1_E270 & El Centro 270 & 0.428 & 0.700 & 0.957 & 3.37 & 0.159 \\
\hline 1_E180 & El Centro 180 & 0.594 & 1.167 & 1.20 & 3.88 & 0.149 \\
\hline 1_CN & TCU075 North & 0.279 & 0.718 & 0.764 & 4.36 & 0.151 \\
\hline 1_CW & TCU075 West & 0.524 & 0.851 & 1.42 & 3.26 & 0.169 \\
\hline
\end{tabular}




\subsection{Phase II USSSI Results Summary}

Provided in Table 5-3 and Table 5-4 are results from Phase II testing. The listed motions are the full $100 \%$ intensity tests using table command signals generated at $60 \%$ intensity levels. These are the most reliable tests from Phase II in terms of replicating the acceleration and absolute displacement characteristics of the desired input motions and are, therefore, used in numerical simulations, which will be discussed in detail in Chapter 6. In Phase II, there is a lower amplitude spectral response at the center surface position $(5 \mathrm{C})$ than at the other instrumented surface positions $(5 \mathrm{M}$ and $5 \mathrm{~W})$. This could be due to container boundary effects, impedance of vertically propagating seismic waves by the structure, and the effect of soil directly above the structure behaving as an attached soil block.

Table 5-3 Summary table of Phase II testing results at the bottom (1C) and top (5C) of the soil column with the embedded model structure

\begin{tabular}{|c|c|c|c|c|c|c|}
\hline \multirow{2}{*}{ Test ID } & \multirow{2}{*}{ Test Motion } & \multicolumn{2}{|c|}{$\begin{array}{c}\text { Peak Accelerations } \\
(\mathrm{g})\end{array}$} & \multicolumn{2}{|c|}{$\begin{array}{c}\text { 5\% Damped } \\
\text { Spectral } \\
\text { Amplitude }(\mathrm{g})\end{array}$} & $\begin{array}{c}\text { Site } \\
\text { Period } \\
\text { (seconds) }\end{array}$ \\
\cline { 3 - 6 } & & $1 \mathrm{C}$ & $5 \mathrm{C}$ & $1 \mathrm{C}$ & $5 \mathrm{C}$ & \\
\hline 2_J000.2 & Joshua Tree 000 & 0.422 & 0.897 & 0.981 & 4.41 & 0.119 \\
\hline 2_J090.2 & Joshua Tree 090 & 0.506 & 0.715 & 1.01 & 4.13 & 0.119 \\
\hline 2_E270.3 & El Centro 270 & 0.484 & 0.635 & 1.32 & 3.30 & 0.105 \\
\hline 2_E180.3 & El Centro 180 & 0.504 & 0.944 & 1.42 & 4.41 & 0.107 \\
\hline 2_CN.2 & TCU075 North & 0.385 & 0.741 & 0.982 & 3.92 & 0.119 \\
\hline 2_CW.2 & TCU075 West & 0.420 & 1.045 & 1.49 & 5.77 & 0.111 \\
\hline 2_CN.3 & TCU075 North & 0.386 & 0.731 & 0.947 & 3.76 & 0.119 \\
\hline 2_CW.3 & TCU075 West & 0.392 & 1.041 & 1.50 & 5.63 & 0.111 \\
\hline
\end{tabular}

The site period is substantially lower in Phase II than in Phase I. As discussed in previous sections, this is probably partially a result of stiffer soil in combination with the inclusion of the structure. The consistency of the site period between both horizontal 
azimuths of each earthquake motion is striking, especially the Joshua Tree motion, which resulted in the exact same period for each Azimuth. Examining the repeated TCU075 tests illustrates the excellent test repeatability. There is a slight decrease in acceleration amplitudes for the repeat TCU075 tests compared to the original tests, which is probably due to minor soil stiffness degradation over the testing period.

Table 5-4 presents maximum racking distortions and acceleration response recorded at the floor and ceiling slabs within the model structure. The racking distortion is the average of the two LVDT recordings relative to the floor slab. Model racking distortion can be used to estimate the implied prototype racking distortion by applying the geometric scale factor $(\lambda)$ of 10 . A discussion on extrapolating racking distortions to the extreme edges of the structure is provided in Chapter 6 along with racking analyses.

Table 5-4 Summary table of Phase II structural racking results

\begin{tabular}{|c|c|c|c|c|c|c|}
\hline \multirow{2}{*}{ Test ID } & \multirow{2}{*}{ Test Motion } & \multicolumn{2}{|c|}{$\begin{array}{c}\text { Peak Accelerations } \\
(\mathrm{g})\end{array}$} & \multicolumn{2}{c|}{$\begin{array}{c}\text { 5\% Damped } \\
\text { Spectral } \\
\text { Amplitude }(\mathrm{g})\end{array}$} & \multirow{2}{*}{$\begin{array}{c}\text { Racking } \\
\text { Distortion } \\
(\mathrm{mm})\end{array}$} \\
\cline { 3 - 6 } & & Floor & Ceiling & Floor & Ceiling & \\
\hline 2_J000.2 & Joshua Tree 000 & 0.438 & 0.624 & 1.63 & 3.46 & 0.628 \\
\hline 2_J090.2 & Joshua Tree 090 & 0.443 & 0.550 & 1.55 & 3.25 & 0.614 \\
\hline 2_E270.3 & El Centro 270 & 0.402 & 0.558 & 1.14 & 2.50 & 0.532 \\
\hline 2_E180.3 & El Centro 180 & 0.393 & 0.695 & 1.13 & 3.30 & 0.720 \\
\hline 2_CN.2 & TCU075 North & 0.472 & 0.545 & 1.46 & 3.12 & 0.555 \\
\hline 2_CW.2 & TCU075 West & 0.434 & 0.722 & 1.74 & 4.25 & 0.804 \\
\hline 2_CN.3 & TCU075 North & 0.451 & 0.525 & 1.39 & 2.99 & 0.547 \\
\hline 2_CW.3 & TCU075 West & 0.428 & 0.683 & 1.77 & 4.16 & 0.803 \\
\hline
\end{tabular}

The higher amplitudes at the ceiling relative to the floor are the result of structural distortion in combination with possible rocking effects, as discussed previously. Similar to the soil acceleration response, repeated TCU075 tests show slightly decreased 
structural accelerations. The difference in recorded racking distortion is slight, but not significant enough to suggest that it is a result of degraded soil in the repeated tests, which would be expected of softer soil based on the racking ratio proposed by J.J. Wang (1993). This further evidences that soil softening over the test period is minor and therefore should not be considered detrimental to the validity of testing results or numerical simulations.

\subsection{Discussion of Experimental Findings}

The experimental test program was very successful in both Phase I free-field testing and Phase II USSSI testing of an embedded model tunnel cross-section. Site characterization illustrated that Phase II contained stiffer soil among the two testing phases, which is reflected in higher spectral amplitudes and a lower site period.

One-dimensional site response was consistent in the overall test program, as evidenced by the motion coherence within individual horizontal planes. Boundary effects tended to increase spectral amplitudes near the extreme edges of the soil column, with no discernable effect on site period. Shifted site periods due to container effects would be much more detrimental to the validity of seismic SSI testing than the slight difference in amplitude. This is especially true for surface structures, where it is particularly important to have knowledge of the site period and natural period of the structure.

High frequency spectral response tends to be somewhat random and possibly affected by signal noise, but there is a secondary resonant spike in many of the motions that may be characteristic of the accelerometer foundations resonating within the soft soil. Rigorous filtering and correction of recorded motions would be required to better 
characterize the high frequency response of the model soil, but such filtering is beyond the scope of this research.

The racking distortions of the model structure are highly dependent on the imposed accelerations, and mostly independent of motion type. The relationship between acceleration and racking distortion is linear, which is likely a result of linear elastic behavior of the acrylic structure. These results cannot be directly applied to other structural configurations, but it is not unreasonable to assume a positive correlation between applied acceleration and distortion for most underground structures. 


\section{CHAPTER 6 - NUMERICAL MODELING}

\subsection{Introduction}

Equivalent linear numerical analyses were performed using the computer codes SHAKE (Schnabel et al. 1972) and FLUSH (Lysmer et al. 1975). The version of SHAKE used in the study is SHAKE91 (Idriss and Sun 1992). Both computer codes compute response in the frequency domain and are linear for any set of material properties. Non-linear behavior is accounted for using an iterative procedure (Schnabel et al. 1972). Both computer programs are established in the field of geotechnical engineering for providing reliable and consistent results.

The results of the computer simulations are presented in this chapter and compared with shake table testing results to assess the performance of the flexible barrel testing platform. Free-field comparisons are presented in the form of 5\% damped response spectra obtained from Phase I free-field testing and SHAKE simulations. Racking distortions obtained from LVDT recordings during Phase II testing are compared with distortions obtained using FLUSH simulations.

\subsection{Model v. Prototype Scale}

All computer simulation results within this paper are presented for the prototype scale. Thus, the pertinent parameters used in the simulations are scaled up using the scale factors provided in Table 3-2 with the geometric scaling factor of 10. Meymand (1998) asserts that "the most accurate use of numerical analysis applied to the modeling process is analysis of the scale model, not to predict the behavior of the implied prototype." This is an important observation and it was taken into careful consideration before choosing to 
run simulations at the prototype scale. The decision to run simulations at the prototype scale, as opposed to the model scale, was motivated by the desire to assess the validity of the scale relations used in the development of the model.

During preliminary SHAKE simulations, both model and prototype scale simulations were performed. Results showed very accurate scaling for quantities important to this study such as spectral accelerations, site periods, shear stresses, and shear strains. The preliminary simulations provided confidence that prototype simulations could be used to gain insight into model behavior at the prototype scale. Nevertheless, caution should be used if the goal of a model test program is to quantitatively predict the true behavior of some full scale structure. As described in Chapter 3, it is unlikely that any set of scaling relations constitute a "true" model. In addition to the conceptual reasoning behind performing simulations at the prototype scale, a technical drawback surfaced in the model scale SHAKE simulations. The SHAKE91 output file resulted in low resolution of the spectral acceleration plots at short periods, because a limited number of high frequency points are output for plotting. Prototype scale simulations provide superior resolution for response spectra by shifting the resonant peaks to longer periods as well as providing more broadband response. FLUSH simulations were performed at the prototype scale for consistency with free-field SHAKE simulations. 


\subsection{Free Field Modeling}

Numerical modeling of the Phase I free-field soil column is very useful for assessing the performance of the testing platform. As previously discussed, a primary goal of the testing container is to provide one-dimensional (1D) site response, so it follows that if the testing results should match reasonably well with SHAKE results if the same input motion is used. The acceleration recording at the table was input as a "within" base motion at bedrock for all numerical simulations.

The equivalent linear method produces reasonably accurate accelerations, stresses, and strains, but velocity and displacement output is much less reliable. Since stress and strain measurements were not recorded in the model soil, all numerical simulations needed to be calibrated against the accelerations recorded in the center accelerometer array. The numerical model was calibrated independently for all six Phase I motions by adjusting the shear wave velocity profile until an optimal spectral response match was achieved between recorded and numerical results within the full depth of the soil column.

\subsubsection{Modeling Parameters}

An advantage of using the equivalent linear method over the non-linear method is that fewer modeling parameters are required for analysis. Only the input motion, initial soil stiffness, and material modulus reduction and damping curves are required for analysis. The input motion can be any recorded acceleration time history input into the model at any desired layer. Soil stiffness is accounted for by inputting unit weight and shear wave velocity profiles. For the shake table tests, the soil unit weight was known, 
and shear wave velocity profiles were derived using T-bar pullout testing and hammer blow testing as described in Chapter 5. It was necessary to calibrate the shear wave velocity profile independently for each motion. Considering the thixotropic nature of the model soil, this may be due to possible modulus degradation and/or strength gain between individual tests; or different dynamic demands from the different motions.

Material curves were not developed specifically for this research, but model soil curves were obtained from Meymand's (1998) pile study. The curves reported in the pile study are referenced from Vucetic and Dobry (1991) and Sun et al. (1988), in addition to a model soil specific curve developed using cyclic triaxial and bender element testing. The Sun et al. (1988) curve is recommended for Young Bay Mud, which is the prototype soil for this study. SHAKE analyses were initially performed using six different sets of curves: the model soil curve (Meymand 1998), the Young Bay Mud (YBM) curve (Sun et al. 1988), and $\mathrm{PI}=30,50,75$, and 100 curves (Vucetic and Dobry 1991).

It was found that the model soil, YBM, and PI=50 curves give nearly identical results for spectral acceleration, shear stress, and shear strain, but at differing levels of modulus degradation. In achieving consistent results, the level of deviation from the measured shear wave velocity profile differs for all three curves and for all six motions. For example, increasing the shear wave velocity by 15 to $30 \%$ was required for the model soil curves to achieve a reasonable spectral match with testing data, which is consistent with Meymand's findings. Conversely, the YBM curves required that the shear wave velocity be decreased by up to $15 \%$ to garner similar results.

The Young Bay Mud curve was used in final SHAKE iterations at the prototype scale, because it provided the most consistent results while requiring the least shear wave 
velocity calibration. Interestingly, the YBM modulus degradation curve matches well with the $\mathrm{PI}=50$ curve, and similarly, the YBM damping curve matches well with the model soil curve. It is important to note that all three of the discussed sets of curves perform well for this research, and final results would not vary significantly regardless of which is chosen. Figure 6.1 displays for comparison the three sets of curves used for initial SHAKE trials.
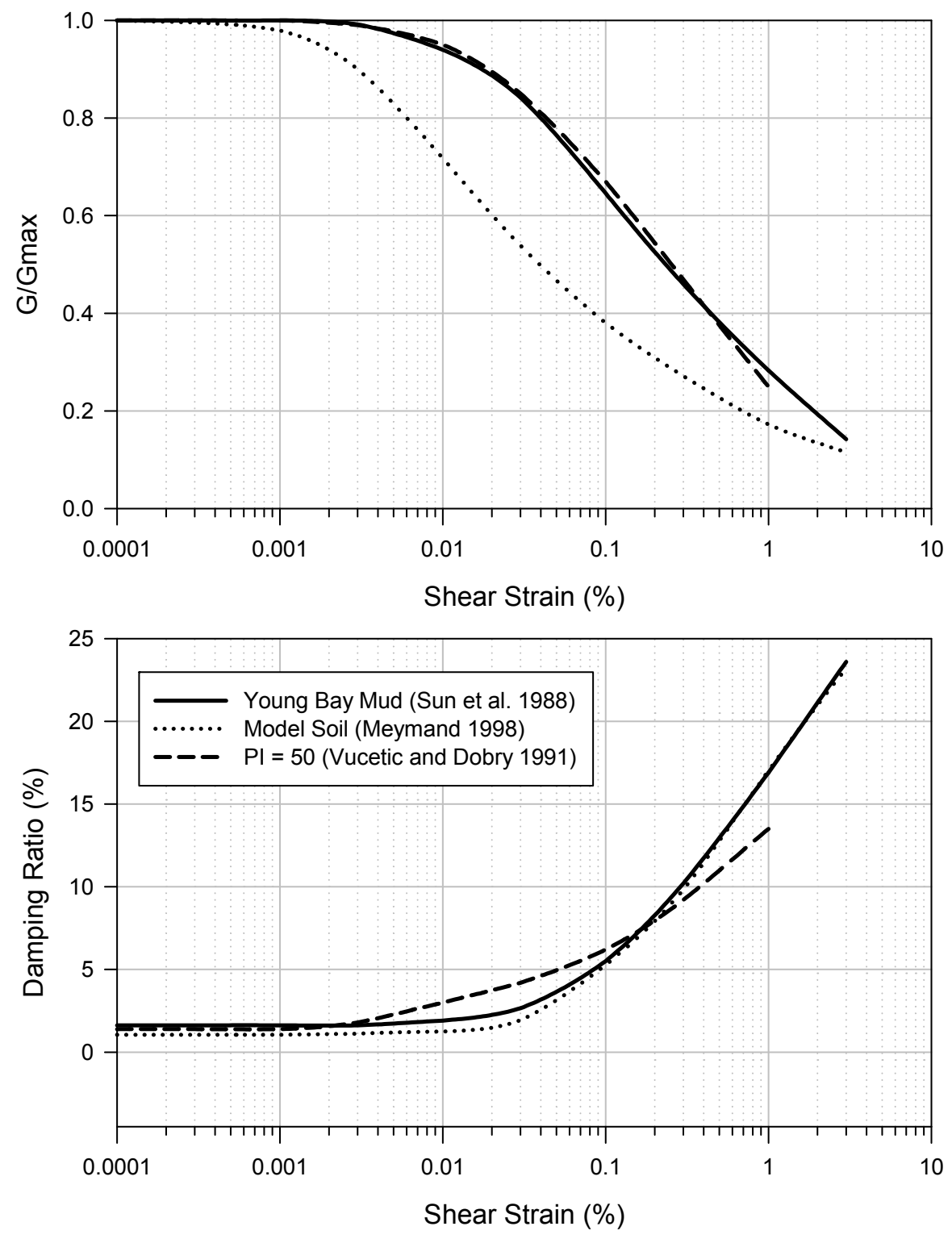

Figure 6.1 - Modulus Reduction and Damping curves used in SHAKE analyses 


\subsubsection{SHAKE Results and Discussion}

This section provides some representative results from SHAKE modeling by comparing 5\% damped response spectra obtained from shake table testing and numerical modeling. Overall, the agreement between Phase I free-field testing and SHAKE simulations is good. The following observations can be made by examining the response spectra for all free-field motions:

- The spectral amplitudes match well at the predominant site period throughout the array for five of the six motions. The exception is the TCU075 West motion in which SHAKE could not produce the full amplitude observed in test 1_CW. This may be due to the large shaking amplitudes which resulted from using the incorrect command signal during testing, as explained in Section 4.9.

- At longer periods, SHAKE captures the shape of the response spectra well, but underestimates the response at shorter periods. This may be a result of soilstructure interaction effects with the soil accelerometers, or of SHAKE not capturing high frequency well. Attempts to run simulations using soil accelerations as input motions garnered less consistent results.

- Test data shows a slightly more broadband frequency range within predominant peaks at the site period than the numerical simulations. This is most apparent by observing the results from the El Centro motions (tests 1_E270 and 1_E280).

- The peak ground acceleration (PGA) is generally underestimated at the soil surface compared to testing data. A closer match is achieved at depth within the soil column. This may be a result of error imposed by the accelerometer foundations, especially near the surface where the foundation was under 
negligible overburden pressure to resist excessive vibration.

- Soil thixotropy may have affected the true soil stiffness between tests. This is most apparent between the TCU075 North (1_CN) and West (1_CW) tests, which were performed consecutively on the same testing day, along with $65 \%$ intensity calibration procedures. The shear wave velocity profile needed to be reduced in SHAKE by $2 \%$ and $14 \%$ for the North and West motions respectively. This suggests that soil stiffness may have degraded over the testing day. Interestingly, the effect is not as apparent for the stiffer Phase II soil column, in which numerical model calibration was much more consistent.

- There is little evidence to suggest that soil column twisting or bending significantly distorted site response at the predominant period, but these deformation modes may be another possible explanation for the high frequency peaks generated from test data. Since SHAKE is a $1 \mathrm{D}$ analysis program, these motions would not be accounted for in simulations.

Based on extensive SHAKE simulations of the Phase I free-field model, it is concluded that the flexible wall test container can adequately simulate free-field response at the predominant period. For the prototype free-field model discussed herein, adequate response is observed at periods greater than about 0.2 seconds. Further work is needed to resolve issues with the short period response, but for the purpose of assessing USSSI effects, high frequency response is less critical. Figure 6.2 shows SHAKE results overlaid with center array testing data for the TCU075 North and El Centro 180 motions. Test results are displayed as black solid lines and SHAKE results as blue dashed lines. Appendix C contains similar response spectra comparisons for all of the Phase I motions. 


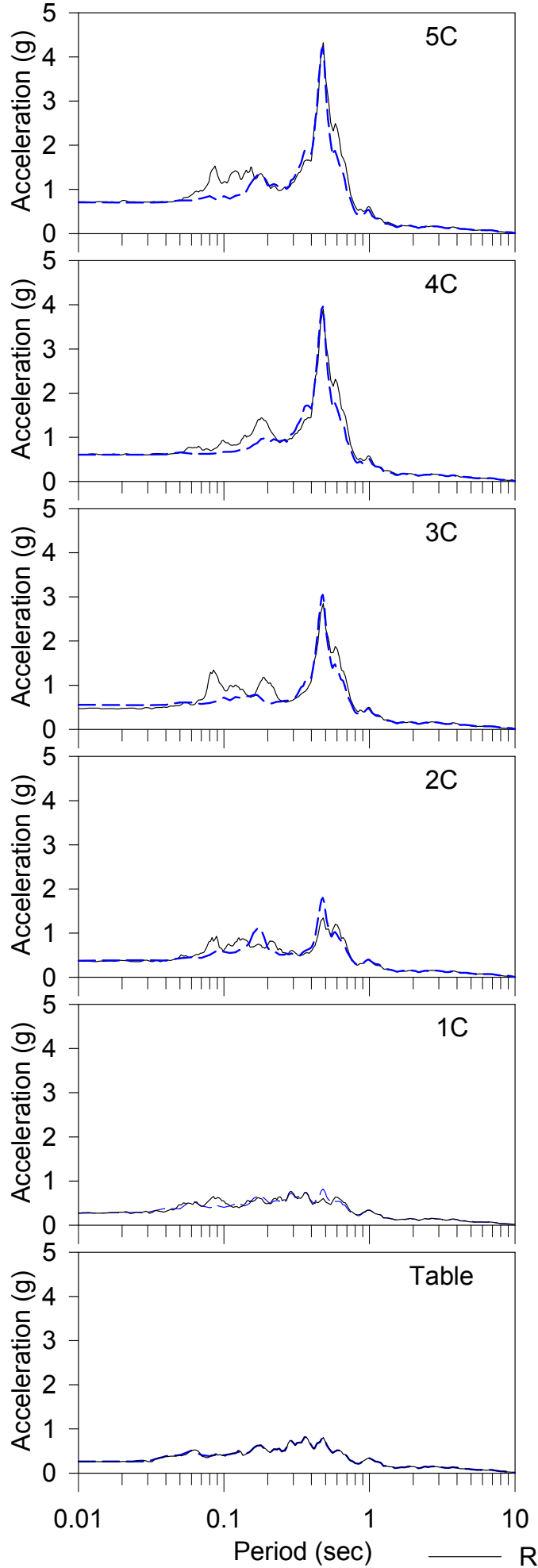

(a)
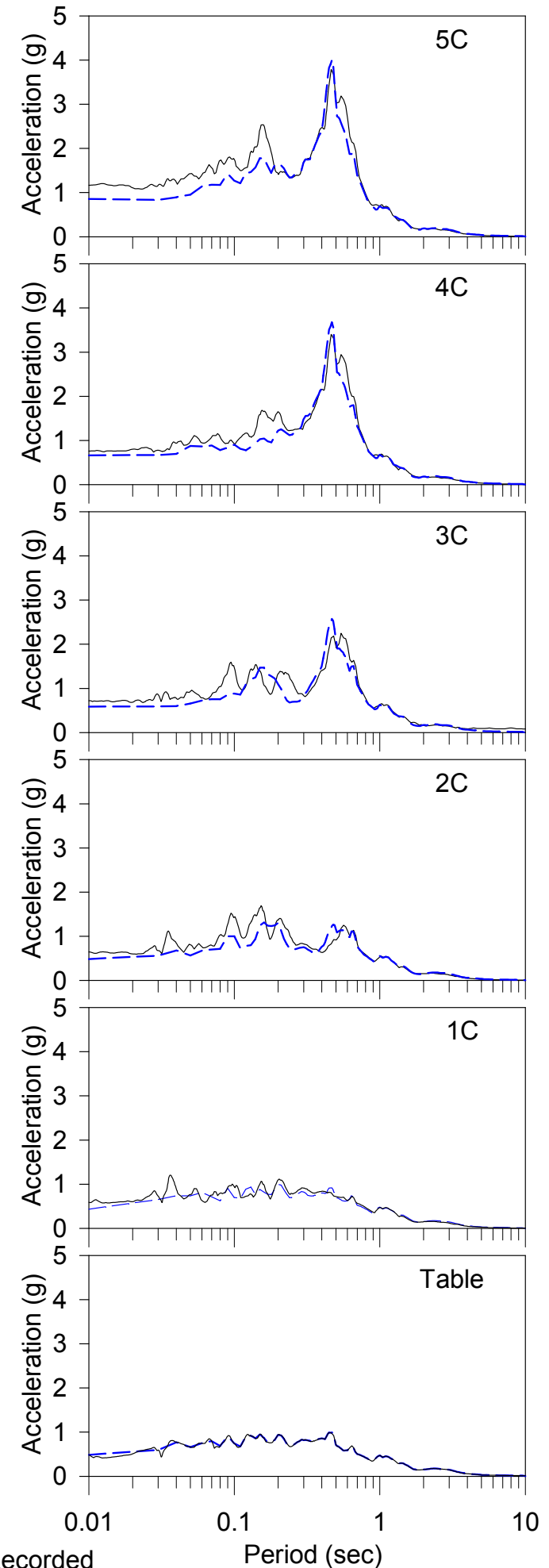

(b)

Figure $6.2-5 \%$ damped response spectra for (a) TCU075 North (1_CN) and (b) El Centro 180 (1_E180) center array recordings versus SHAKE predictions 


\subsubsection{Free-Field Racking Analysis}

Simple racking analyses for any underground circular or rectangular structure can be performed using methods recommended by J. J. Wang (1993) in conjunction with SHAKE results. The following describes a simple approach for deriving free-field and structural racking distortions.

1. Free-field shear strains and strain compatible soil stiffness are obtained over each layer in the SHAKE model (total of 49 layers).

2. Evaluate the flexibility ratio $(F)$ at each layer using the strain compatible soil stiffness (from SHAKE results) and the stiffness of the proposed structure with equation 3.14 .

3. The layer specific racking ratio $(R)$ is obtained using the flexibility ratios and the normalized racking relationship in J. J. Wang (1993) (Figure 2.3). For this research, a $3^{\text {rd }}$ order polynomial equation was fit over the relevant data range for ease of spreadsheet calculations (Figure 6.3).

4. Structural shear strain at any given layer is obtained by multiplying the racking ratio by the free-field strain at that layer.

5. The estimated racking distortion is the average of the layer specific structural shear strain multiplied by the height of the structure.

Other approaches such as simply performing the analysis at the midpoint of the structure provide similar results. This type of analysis is based on assumptions presented in J. J. Wang (1993), and deviation from those assumptions may limit the applicability of the method. For example, the depth of soil overburden should be greater than or equal to the height of the structure to limit surface boundary effects. This condition is not satisfied in 
this research, resulting in possible overestimation of structural racking. Considering this minor deviation from Wang's assumptions, the analysis is still more accurate than a simple free-field deformation method for the structure in this study. Results of the analysis are provided in Table 6-1 for all Phase I free-field test motions.

Table 6-1 Results of simplified racking analysis using SHAKE and the racking relationship in Figure 6.3

\begin{tabular}{|c|c|c|c|c|c|}
\hline Test ID & Test Motion & $\begin{array}{c}\text { Flexibility } \\
\text { Ratio }\end{array}$ & $\begin{array}{c}\text { Racking } \\
\text { Ratio }\end{array}$ & $\begin{array}{c}\text { Free-Field } \\
\text { Deformation } \\
(\mathrm{mm})\end{array}$ & $\begin{array}{c}\text { Racking } \\
\text { Distortion } \\
(\mathrm{mm})\end{array}$ \\
\hline \hline 1_J000 & Joshua Tree 000 & 0.26 & 0.36 & 37 & 13 \\
\hline 1_J090 & Joshua Tree 090 & 0.29 & 0.39 & 18 & 7 \\
\hline 1_E270 & El Centro 270 & 0.30 & 0.40 & 22 & 9 \\
\hline 1_E180 & El Centro 180 & 0.28 & 0.37 & 32 & 12 \\
\hline 1_CN & TCU075 North & 0.31 & 0.41 & 28 & 12 \\
\hline 1_CW & TCU075 West & 0.22 & 0.31 & 33 & 10 \\
\hline
\end{tabular}

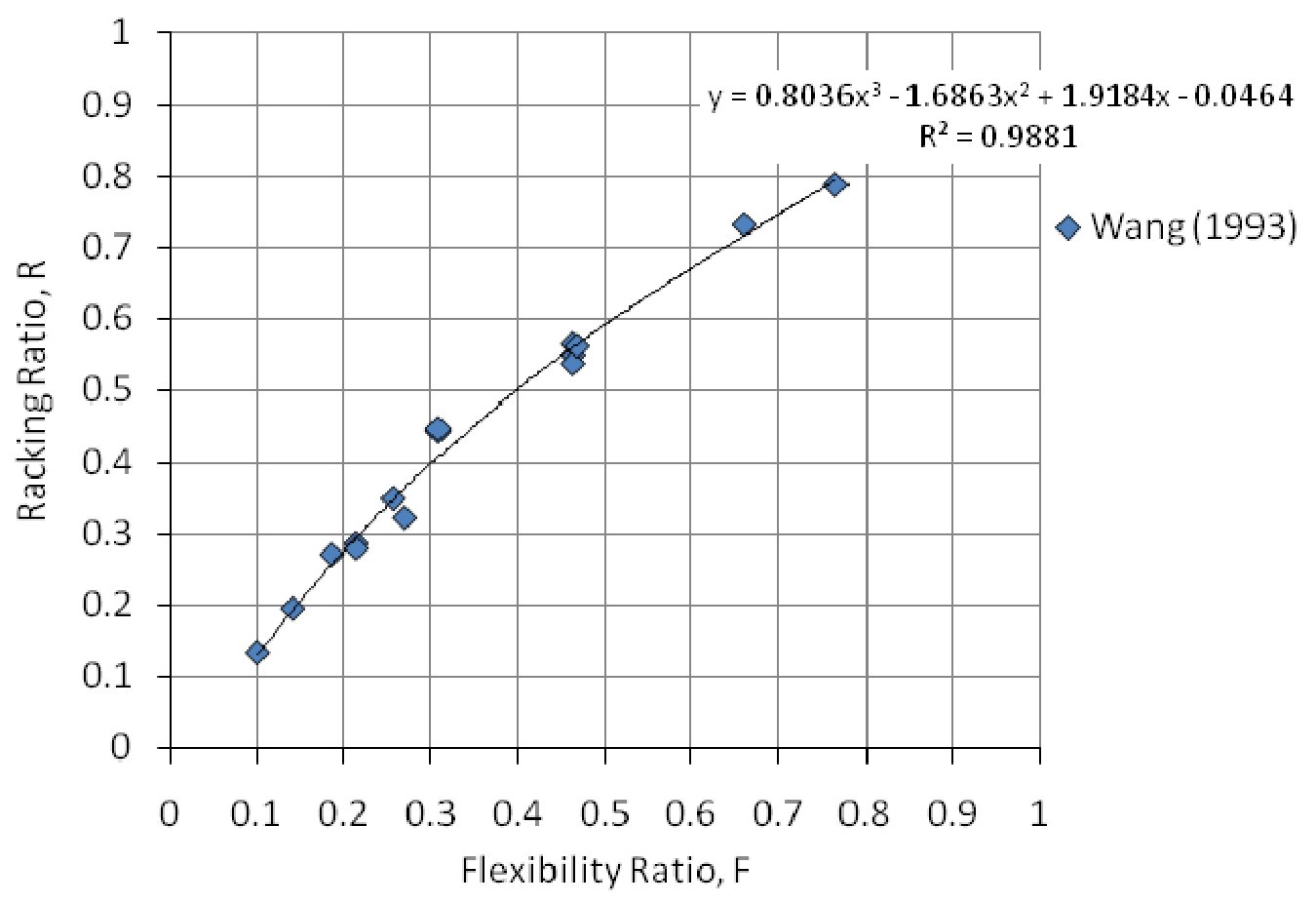

Figure 6.3 - A portion of the normalized racking relationship developed by J. J. Wang (1993) with a curve fit for use in a spreadsheet solution 


\subsection{USSSI Modeling using FLUSH}

The two-dimensional plane strain equivalent linear computer program FLUSH was used to numerically model the USSSI effects for the Phase II soil column with the embedded model structure. The main purpose of the USSSI modeling in this study is to extract racking displacements of the underground structure for comparison with actual racking measurements taken during Phase II testing. Similar to the SHAKE simulations, all USSSI simulations were conducted at the prototype scale. The method of implied prototypes was used in the development of the model for both soil and structural properties in order to limit the error induced in the scaling process. The possibility of running FLUSH simulations at the model scale was explored, but ultimately abandoned due to program limitations at small time steps.

\subsubsection{Modeling Parameters and Methodology}

The first step to creating a suitable FLUSH model is to build a free-field mesh and compare free-field results with Phase I SHAKE results. The finite element (FE) mesh and its associated layer thicknesses were built so as not to alias frequency content lower than $15 \mathrm{~Hz}$, while still allowing for reasonable computation time. The same modulus reduction and damping curves used in SHAKE are also used in all FLUSH analyses. Free-field FLUSH results using the proposed finite element mesh compared relatively well with the SHAKE results for both 5\% damped response spectra and free-field deformations. This provided confidence in the suitability of the FE mesh for further USSSI modeling. 
The USSSI FE mesh was developed using the same dimensions as the free-field mesh, but a rectangular section of soil was replaced by the box subway cross-section. Keeping in mind the method of implied prototypes, the material properties of acrylic were scaled up for use in the prototype model in lieu of specifying reinforced concrete properties. For example, the density of acrylic was specified in the model keeping in mind the material density scaling factor of one. All properties were scaled using the scaling relations provided in Table 3-2. Dimensionless material properties of acrylic were also scaled by a factor of one, such as the material damping and the Poisson's ratio. The material damping and Poisson's ratio of acrylic were selected as reported by Buehrle et al. (2003), who performed a study on the damping characteristics of Plexiglas windows. The Poisson's ratio $(v)$ is used in FLUSH to calculate the flexural modulus $(E)$ from shear modulus $(G)$ which is input by the user. Thus, if a design flexural modulus is required, the shear modulus must first be evaluated using the following equation:

$$
G=\frac{E}{2(1+v)}
$$

Prototype scale material properties of the structure for use in FLUSH simulations are summarized in Table 6-2 and are compared with typical properties for reinforced concrete (Hassoun 2001; Morante 2006).

Table 6-2 Material properties of the subway cross-section for use in FLUSH

\begin{tabular}{|l|c|c|}
\hline Material Property & FLUSH Model & Reinforced Concrete \\
\hline \hline Unit weight $\left(\mathrm{kN} / \mathrm{m}^{3}\right)$ & 11.8 & 22.0 to 25.1 \\
\hline Poisson's Ratio & 0.35 & 0.15 to 0.20 \\
\hline Damping Ratio & 0.035 & 0.04 to 0.07 \\
\hline Shear Modulus $(\mathrm{MPa})$ & 12,100 & 10,000 to 16,000 \\
\hline Flexural Modulus $(\mathrm{MPa})$ & 32,800 & 20,000 to 36,000 \\
\hline
\end{tabular}


First iteration soil properties were selected using the shear wave velocity profile developed for the Phase II soil column (Figure 5.3). The profile was scaled to that of the prototype and subsequently converted to soil stiffness using the following equation:

$$
V_{s}=\sqrt{\frac{G_{s}}{\rho_{s}}}
$$

where $V_{s}$ is shear wave velocity, $G_{s}$ is soil stiffness, and $\rho_{s}$ is soil density. The Poisson's ratio for the clay was selected as 0.45 based on recommendations in Bowles (1995). The FLUSH model was calibrated against the $5 \%$ damped response spectra generated from the top center soil accelerometer (5C) recording. The soil stiffness profile was modified over multiple FLUSH simulations until an adequate response spectra match with test results was achieved. Increases of 5 to $6 \%$ from the first iteration stiffness profile resulted in very good matching of 5\% damped response spectra between the Phase II test results and the FLUSH model for all six full intensity motions (tests 2_J000.2, 2_J090.2, 2_E270.3,2_E180.3,2_CN.3, and 2_CW.3). This is much more consistent than Phase I, in which the shear wave velocity profile had to be adjusted over a much wider range to provide adequate results. These results may indicate that the stiffer Phase II soil column was more stable and less affected by modulus degradation and soil thixotropy than the softer Phase I soil profile.

To calculate racking distortions, fictional diagonal members that are weak in the axial direction were added to the structure to act as strain gauges. This method was employed because FLUSH provides relatively accurate stress and strain results, but absolute displacement calculations are unreliable. This prohibits accurate calculation of 
differential displacement between nodes using absolute displacements. According to Lysmer et al. (1975), an auxiliary program for calculating relative displacement time histories between nodes is available, but it is not utilized in this study. The strain gauge member stiffness was specified to be very low so that the response of the structure is not affected. Similar diagonal members were used in the free-field FLUSH model to characterize the free-field deformations with the same initial soil stiffness profile as in the USSSI model. The following equations illustrate the process of converting the calculated axial load in the strain gauge members to racking distortion.

$$
\begin{gathered}
\Delta_{L}=\frac{F L}{A E} \\
\Delta_{R}=\frac{\Delta_{L}}{\cos (\alpha)}
\end{gathered}
$$

Where: $\Delta_{\mathrm{L}}=$ axial deformation, $\mathrm{F}=$ axial force, $\mathrm{L}=$ length, $\mathrm{A}=$ cross-sectional area, $\mathrm{E}=$ Young's modulus, $\Delta_{\mathrm{R}}=$ horizontal racking, and $\alpha=$ angle formed between the structural floor and the strain gauge member or the diagonal angle of the structural opening.

Distortions imposed on the structure are small relative to the dimensions of the tunnel opening, which permits the use of $\alpha$ based on the small angle approximation. The very slight angle change resulting from the small structural distortion is negligible and to consider it would unnecessarily complicate the analysis. Figure 6.4 displays the finite element mesh used in the USSSI analysis and includes the diagonal strain gauge members. The free-field mesh has the same dimensions, but the structure and tunnel opening is replaced with soil elements. 
Both the USSSI and free-field finite element meshes are used in the racking analysis presented in the following sections. Horizontal racking deformation is evaluated using the strain gauge members, and the racking ratio $(\mathrm{R})$ is computed as the ratio of the tunnel deformation to the free-field deformation. The flexibility ratio $(\mathrm{F})$ is evaluated using equation 3.14, which requires the input of soil stiffness and structural stiffness. Free-field FLUSH simulations provide the strain-compatible shear modulus $\left(\mathrm{G}_{\mathrm{s}}\right)$ for all soil elements over the depth of the structure, and the average of these values is used in the analysis. Structural stiffness $\left(\mathrm{S}_{1}\right)$ is obtained using equation 3.15 , which is a simplified equation for the stiffness of a box frame with equal flexural rigidity (EI) for the slabs and walls.

Racking distortions from FLUSH simulations are compared with the racking distortions obtained through testing. Due to the geometric limitations of the physical model, the measurements do not provide the racking distortion at the extreme top and bottom edges of the section. This is contrary to the strain gauge members in the numerical model which span the diagonal of the entire structure. For better comparison with FLUSH results, the maximum racking distortion values recorded during testing are extrapolated linearly to the extreme edges. This is thought to be a conservative methodology since the true deformational shape of the structure is not linear, and most of the deformation occurs between the ceiling and floor slabs. 


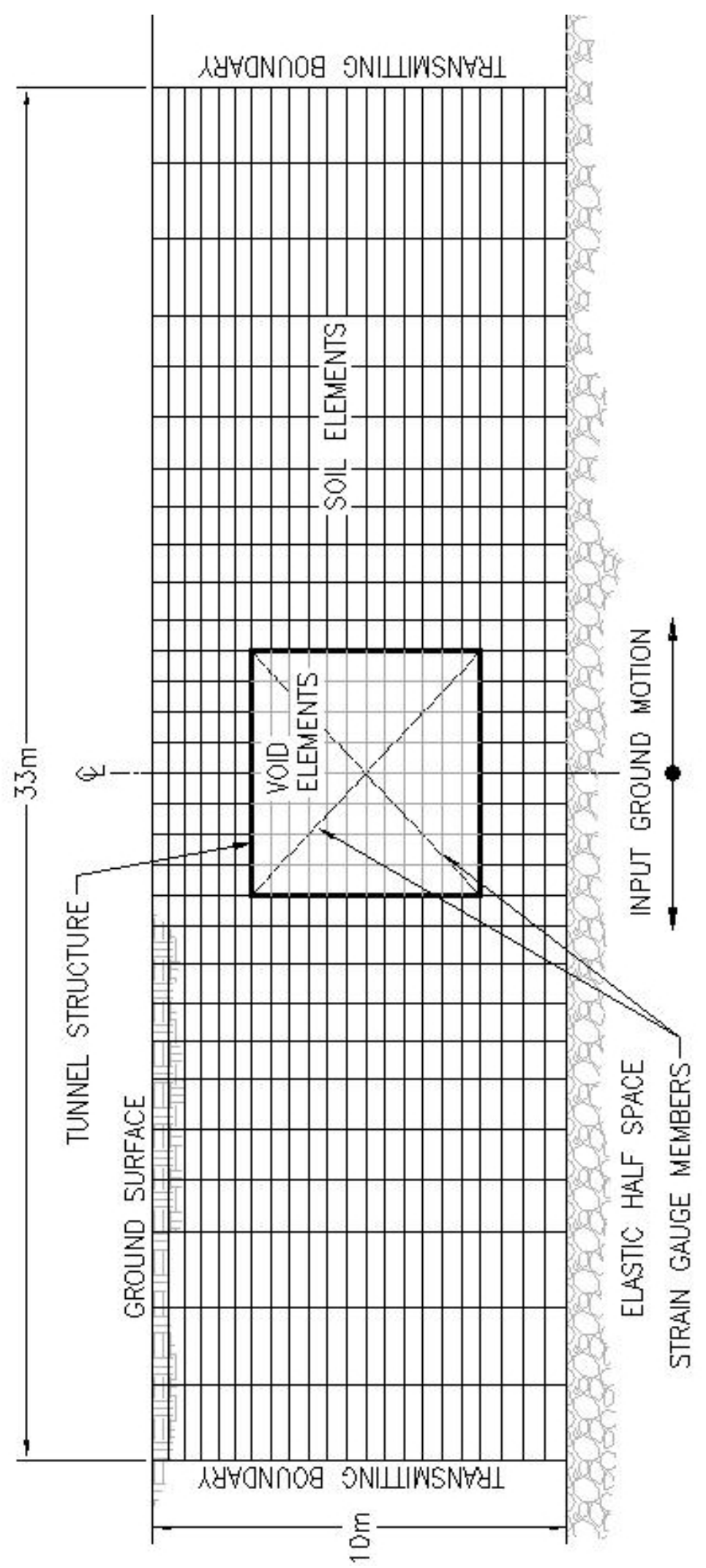

Figure 6.4 - Finite element mesh used for USSSI simulations of the Phase II testing 


\subsubsection{FLUSH Results and Discussion}

The 5\% damped response spectra from Phase II model tests match very well with results from FLUSH simulations, especially at the soil surface. This is also true for the peak ground acceleration and predominant site periods. These observations are illustrated by Figure 6.5 which shows the 5\% damped response spectra recorded at the soil accelerometer positions $1 \mathrm{C}$ and 5C, compared with numerical results from free-field and USSSI flush models.

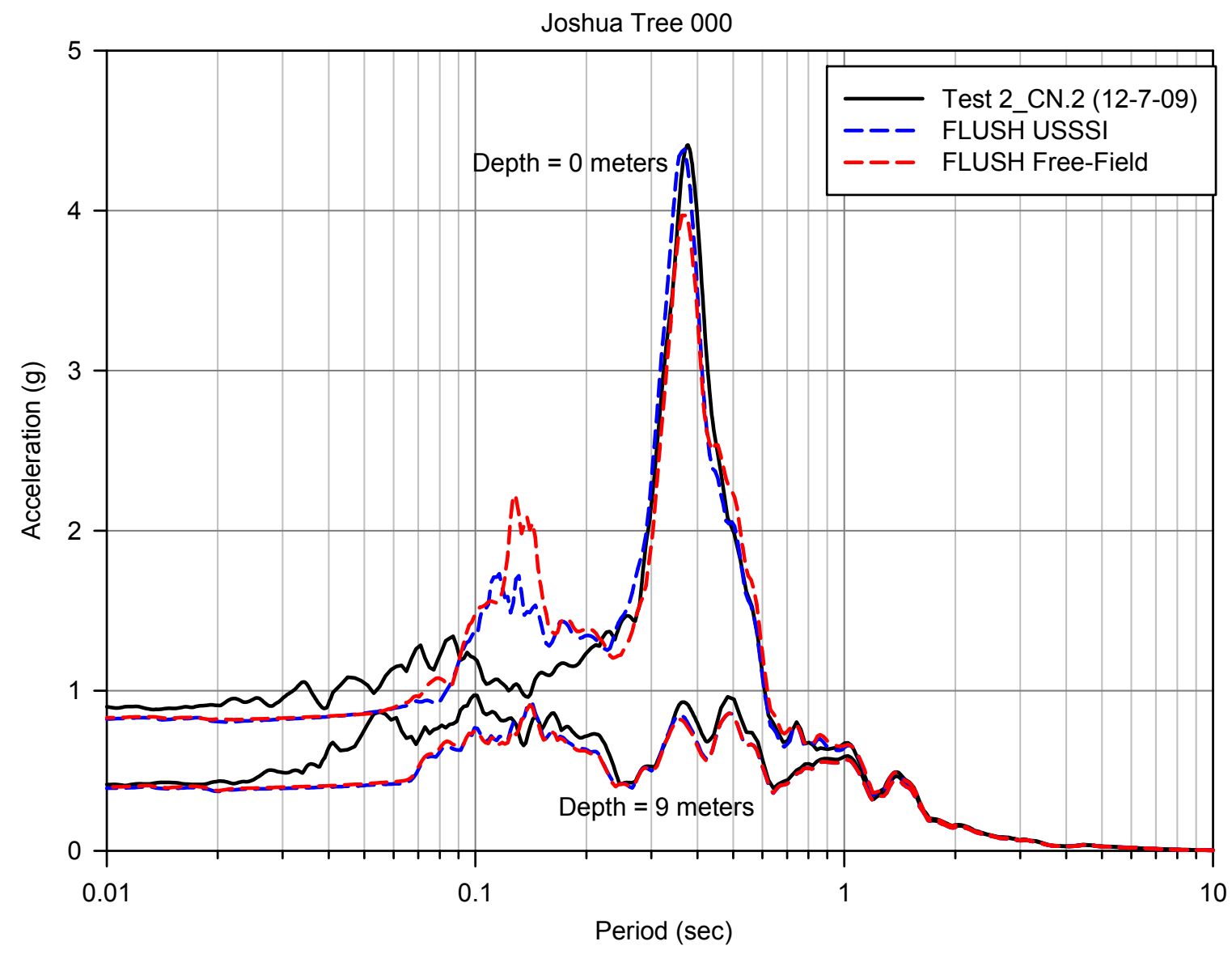

Figure 6.5 - 5\% Damped response spectrum from test 2_J000.3 compared with free-field and USSSI test results 
It is apparent in Figure 6.5 that the inclusion of the structure in the FLUSH model is accompanied by an increase in spectral amplitude. Interestingly, there is negligible period shifting between the free-field and USSSI models. There is also a secondary short period mode in the numerical response spectrum that is not as prominent in the test data. It is important to note that this pattern contradicts Phase I results in which the test data provided higher amplitude short period peaks than the numerical simulations using SHAKE. Further research may be needed to evaluate the high frequency behavior of the test container and the accelerometer foundations. These general patterns of spectral response are typical of all of the six FLUSH simulations.

The main goal of USSSI modeling in this study is to evaluate racking deformations and make comparisons with the actual racking distortions recorded during shake table testing. Table 6-3 shows this comparison for all six motions at the prototype scale. The adjusted values represent distortion recordings that are linearly extrapolated to the extreme top and bottom of the test structure (outside dimensions) based on the LVDT position and the thickness of the floor and ceiling slabs.

Table 6-3 Racking distortion comparison of actual test data with FLUSH simulations

\begin{tabular}{|c|c|c|c|c|c|}
\hline \multirow[b]{2}{*}{ Test ID } & \multirow[b]{2}{*}{ Motion } & \multicolumn{3}{|c|}{ Racking Distortions $\left(\Delta_{R}\right)$} & \multirow{2}{*}{$\begin{array}{c}\text { Ratio } \\
\text { FLUSH/Actual }\end{array}$} \\
\hline & & $\begin{array}{l}\text { Actual } \\
(\mathrm{mm})\end{array}$ & $\begin{array}{l}\text { Adjusted } \\
\text { (mm) }\end{array}$ & $\begin{array}{c}\text { FLUSH } \\
(\mathrm{mm})\end{array}$ & \\
\hline $1 \mathrm{~J} 000$ & Joshua Tree 000 & 6.28 & 7.93 & 12.67 & 2.02 \\
\hline $1 \mathrm{~J} 090$ & Joshua Tree 090 & 6.14 & 7.75 & 12.16 & 1.98 \\
\hline 1_E270 & El Centro 270 & 5.32 & 6.72 & 10.21 & 1.92 \\
\hline 1 E180 & El Centro 180 & 7.20 & 9.09 & 13.28 & 1.84 \\
\hline $1 \_\mathrm{CN}$ & TCU075 North & 5.47 & 6.90 & 9.55 & 1.75 \\
\hline 1 & TCU075 West & 8.03 & 10.14 & 18.35 & 2.29 \\
\hline
\end{tabular}


Examining Table 6-3, the FLUSH model overestimates racking distortions by a factor of two (on average) when compared to the actual unadjusted racking distortions. Even after adjusting the recorded racking distortions, this factor is still about 1.6 on average. These results suggest that the method of analysis used in FLUSH may be conservative in situations where a stiff rectangular structure is embedded in soft clay. At least two reasons for this may exist. First, the distortion at the extreme top and bottom of the structure is hindered by the relatively thick floor and ceiling slabs, as most of the bending and shear distortion takes place between the slabs. Huo et al. (2006) echoes similar conclusions in the formulation of his analytical model suggesting that "for practical purposes the 'effective' structure dimensions are those of the opening." Second, the FLUSH model treats all structural members as linear bending members, where model compatibility is only considered at nodes. Thus, the tunnel opening in the FLUSH model spans the outside edge of the structural geometry and bending and shear distortion is taking place over the entire height of the structure. Neither FLUSH nor the simplified analytical formulation for structural stiffness (equation 3.15) take into account the deformational limitations imposed by the relatively thick structural members. In summation, the actual model structure's linear elastic racking stiffness is possibly higher than specified in the design, due to the relatively thick floor and ceiling slabs impeding distortion.

Despite the discrepancy between testing data and numerical results, there is a consistent pattern. This pattern can be illustrated by normalizing the racking distortions from testing data and FLUSH against their respective averages over the six tests. This procedure aids direct comparison of test results with numerical results. Figure 6.6 shows 
the average normalized results, and it is apparent that the distortion levels follow a consistent trend between numerical and test results for any given motion. For example, relatively high recorded test distortions accompany higher numerical distortions, and similarly, low test distortions accompany low numerical distortions. The Joshua Tree test motions in particular illustrate the consistency between test and numerical results.

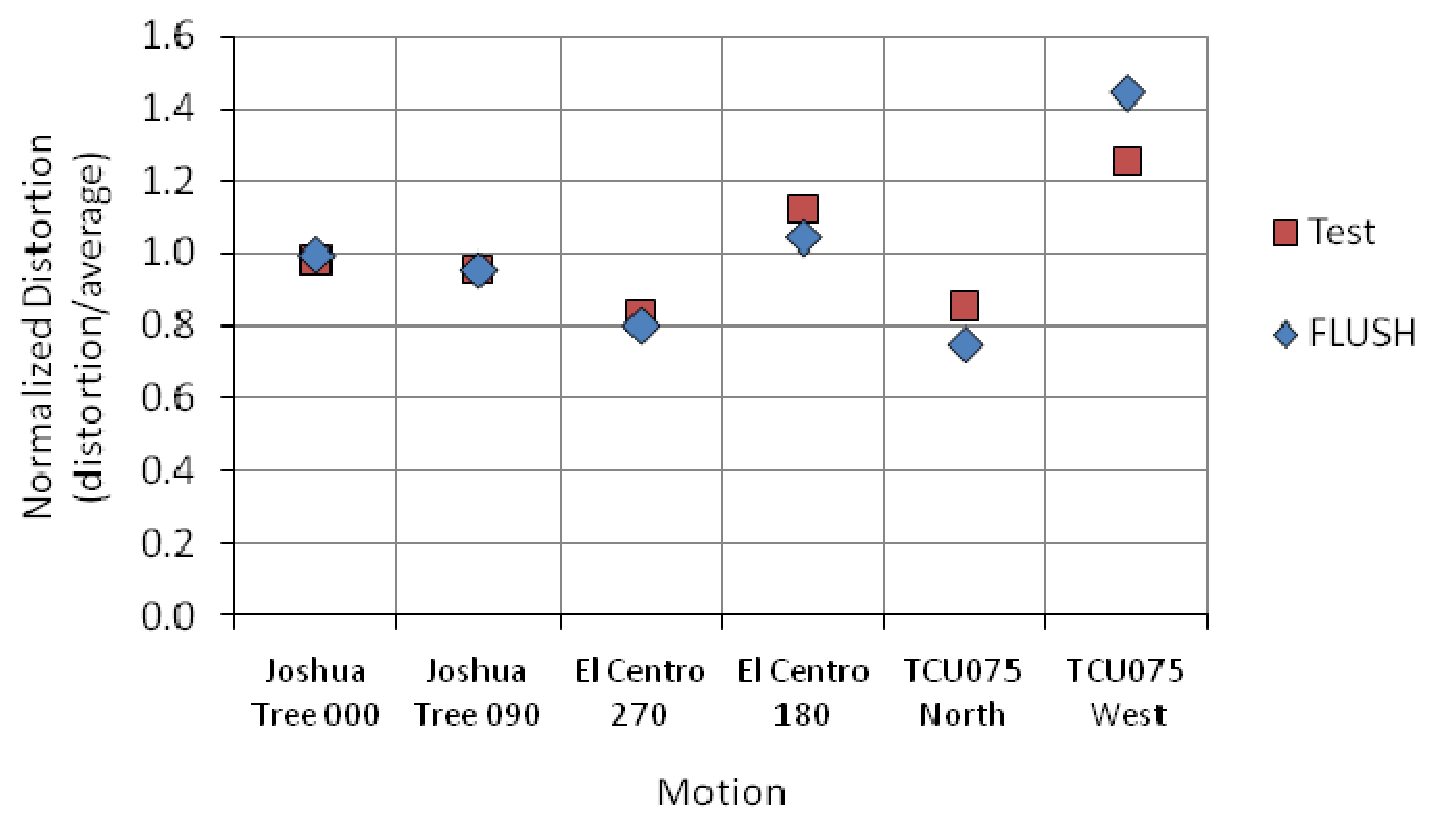

Figure 6.6 Comparison of testing and FLUSH racking distortions, normalized by the average values over all motions

Table 6-4 contains distortions obtained from the free-field finite element analysis for all six motions. Using the free-field distortions and the structural racking distortions in Table 6-3, the flexibility and racking ratios can be evaluated using the methods described in the previous section. Table 6-4 summarizes the normalized racking values and Figure 6.7 is a plot comparing the results from this study to that of J. J. Wang (1993). The FLUSH results in this study compare well with the results from the previous study, aside from a slight overestimation for the TCU075 West motion. Comparing the adjusted 
test results against numerical results again illustrates the overestimation of racking distortions obtained using FLUSH. It is important to note that the flexibility ratio remains the same because it is based on the simplified equation for structural stiffness (equation 3.15). It probably does not reflect the true stiffness as a result of the relatively thick sidewalls and slabs. More rigorous structural analysis or experimentation may be useful to further characterize the stiffness and response of the structure.

Table 6-4 Summary of normalized racking distortions

\begin{tabular}{|l|l|c|c|c|c|}
\hline \multirow{2}{*}{ Test ID } & \multirow{2}{*}{ Motion } & \multirow{2}{*}{$\begin{array}{c}\text { Free-field } \\
\text { distortion }(\mathrm{mm})\end{array}$} & \multicolumn{2}{|c|}{ Racking Ratio, R } & Flexibility \\
\cline { 4 - 5 } & & 18.99 & 0.417 & 0.667 & 0.520 \\
\hline 1_J000 & Joshua Tree 000 & 19.64 & 0.395 & 0.619 & 0.513 \\
\hline 1_J090 & Joshua Tree 090 & 13.99 & 0.480 & 0.729 & 0.568 \\
\hline 1_E270 & El Centro 270 & 19.66 & 0.462 & 0.676 & 0.514 \\
\hline 1_E180 & El Centro 180 & 14.31 & 0.483 & 0.668 & 0.551 \\
\hline 1_CN & TCU075 North & 23.20 & 0.437 & 0.791 & 0.477 \\
\hline 1_CW & TCU075 West & &
\end{tabular}

* From actual test data extrapolated to account for the full structural height

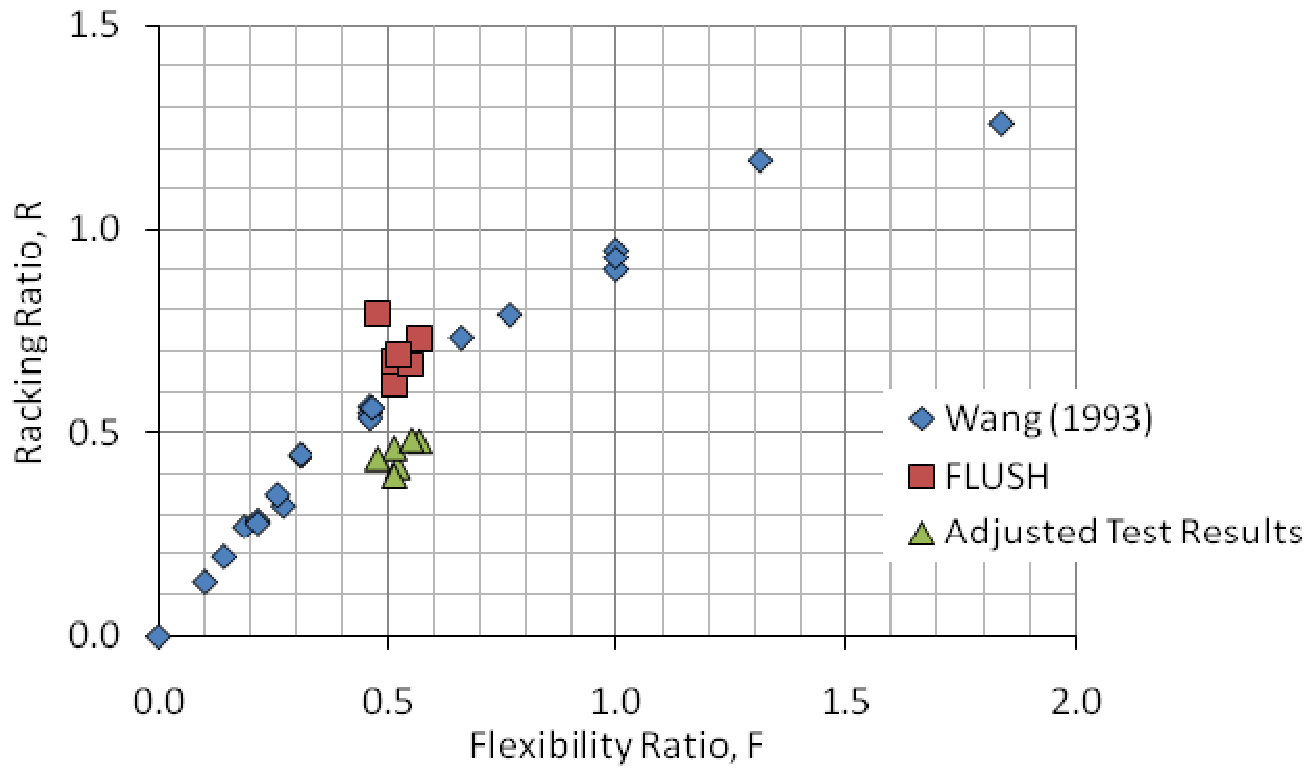

Figure 6.7 - Normalized racking distortions obtained from numerical analysis (FLUSH) and test results (adjusted) compared with those from J. J. Wang (1993). 


\subsection{Discussion of Numerical Results}

Overall, the equivalent linear numerical simulations were very successful for both Phase I free-field simulations and Phase II USSSI simulations. The following are some important points that can be inferred by observing the numerical results as a whole:

- The relatively minor increase in stiffness from Phase I to Phase II resulted in a large decrease in the predominant period. The FLUSH results imply that the period shift between the two testing phases is the result of increased soil stiffness, not the addition of a structure. Another explanation is that FLUSH may not be capable of capturing the all of the non-linear effects associated with the inclusion of the structure, such as the relationship between the soil-structure interface shear friction and modulus degradation described in Huo et al. (2005).

- Both SHAKE and FLUSH do not accurately model the short period response of the test container, but predominant and long period spectral matching is excellent. This is probably a result of deficiencies of the testing platform.

- Peak ground acceleration is more accurately modeled in the Phase II SSI model, which indicates that the stiffer system may be more stable and less prone to spurious high frequency content.

- FLUSH may overestimate the structural racking compared to the physical structure if geometric limitations such as thick slabs are not considered in the preliminary structural analysis. This indicates that FLUSH results are conservative in the case of a stiff structure in soft clay using the simple structural analysis methods presented in this study. More complex structural analysis 
methods that carefully consider structural geometry may provide more accurate results.

- In addition to the physical testing results, numerical results provide further evidence that the flexible model test container can adequately model onedimensional site response, especially at longer periods.

- Racking analyses from both SHAKE and FLUSH illustrate the merits of considering seismic SSI effects in design of stiff cut-and-cover structures in soft soil. The free-field deformation method is experimentally and numerically shown to be highly conservative based on Phase II testing and FLUSH analyses respectively. 


\section{CHAPTER 7 - SUMMARY AND CONCLUSIONS}

\subsection{Project Scope and Summary}

Many existing tunnels in seismically active regions have been designed and built without consideration of seismic soil-structure interaction (SSI) effects. An infamous example is the Daikai subway station which collapsed during the 1995 Kobe Earthquake in Japan. Other shallow cut-and-cover tunnels have been designed using overly conservative methods such as the Mononobe-Okabe method or the free-field deformation method. Current state of practice promotes underground structure design using empirical and analytical relationships developed using numerical modeling. Unfortunately, empirical data for calibrating numerical models regarding SSI effects is limited for tunnels, especially in cohesive soils.

A suitable testing platform was developed to explore the coupled USSSI effects on underground structures in soft cohesive soil. The testing platform consists of a flexible walled testing container founded on a shake table that allows simulation of onedimensional simple shear response in a 1-g testing environment. Horizontal racking deformations for a stiff rectangular tunnel cross-section subjected to various strong ground motions in the transverse direction were measured. Consideration of scale model similitude was of utmost importance in the development of the model in order to gain insight into prototype behavior

Based on test results and equivalent numerical analyses, the testing platform is shown to be a practical and effective means for running physical SSI experiments at model scales. This chapter summarizes important research findings gained through experimental shake table testing and numerical analyses. Also outlined are 
recommendations for improvement of the testing platform and possibilities for future research.

\subsection{Research Findings}

Free-field site response results and associated SHAKE modeling suggest that the flexible wall test container can reasonably mimic one-dimensional free-field conditions for the simple shear deformation mode. Similarly, the site response agreement between FLUSH and shake table results suggest that the container is also capable of adequately modeling soil-structure systems. Also, Phase II repeat tests of the TCU075 motions indicate that the testing container provides sufficient test repeatability.

The scale model similitude effort was successful in modeling prototype behavior of Young Bay Mud at the model scale, as evidenced by the excellent site response match between model scale test results and prototype scale numerical results.

Deformation modes such as soil column twisting or bending cannot be modeled using plane strain methods, and thus are not quantified in this study. There is little evidence to suggest that these deformation modes had significant influence on important test results such as horizontal racking deformations. Further research may be needed to quantify the effect of the unwanted deformation modes on site response and structural response

Simple shear deformation of the model tunnel structure is apparent by comparing the LVDT recordings, which illustrate equal distortion at each sidewall at any given moment in the time history. Furthermore, there is linear relationship between maximum differential racking acceleration and maximum racking distortion. This is reminiscent of 
linear elastic structural response, in which deformation is proportional to applied load. Although this seems intuitive, it is an important experimental finding, because there is often a disconnect between acceleration and displacement in many geotechnical seismic applications.

Consistent with observations from earlier works, rigid body rotation of the tunnel cross-section appears to accompany racking deformations (Penzien 2000; J. J. Wang 1993). Rigid body rotation is not a significant concern under plane strain conditions, but may be important when considering the three-dimensional effects along tunnel alignment.

Some non-linear effects regarding USSSI response do not seem to be captured through equivalent linear numerical analysis. It is possible that the near-field soil surrounding the tunnel behaves as an attached soil block, which is dependent on the adhesion at the soil-structure interface. This effect was modeled by Huo et al. (2005) using non-linear finite element analyses, showing that the extent of modulus degradation of near-field soil is partly a function of the adhesion at the soil-structure interface.

The results presented in this study reinforce the importance of considering kinematic interaction for underground structures. The stiffness contrast between the stiff structure and the soft clay resulted in small structural distortions compared to corresponding free-field shear distortions, as predicted using SHAKE and FLUSH. Other than intensity, varying earthquake motions appears to have little influence on the kinematic response of the structure. This conclusion is based on the consistency of normalized racking distortions for all six motions.

It is clear that the free-field deformation method is highly conservative for stiff structures. Further, test results indicate that conventional empirical and analytical tools 
for evaluating racking distortion may also overestimate racking distortions to some extent using simple structural analysis methods. It appears that simplified equations for stiffness of a rectangular cross-section may underestimate stiffness in cases where the wall and slabs are relatively thick compared to the outside dimensions of the structure. This is because most shear and bending distortion takes place between the thick orthogonal slabs, essentially limiting the distortion based on the geometry of the tunnel opening.

An area of further study regarding the testing platform is in the short period response. Modeling of longer period effects, such as the primary shear deformation mode, is promising, but short period response may be adversely affected by a series of possible issues. These issues include but may not be limited to: soil-accelerometer interaction, unwanted soil column deformation modes, spurious high frequency content, and undesired shake table motions. The contribution of high frequency content on radiation damping effects is a final issue which may warrant consideration, but is beyond the scope of this research.

\subsection{Improvement of the Testing Platform}

It is the opinion of the author that the testing platform developed in this study is a useful tool for future research regarding seismic soil-structure interaction problems. Considering this, there is room for improvement in different aspects of the testing program. The following are some recommendations for improvement:

- Soil mixing and placement procedures should be improved to provide more homogeneous soil properties. Use of paddle mixers within the tank during 
circulation would help to achieve this result, especially during the process of adding fly ash.

- Improved accelerometer foundations and placement procedures may help to limit possible soil-accelerometer interaction effects.

- Placement of multiple vertical accelerometers within the soil column, on the model structure, and on the shake table would be useful in identifying rocking or bending modes. The effects and causes of soil column twisting is also an area of further study.

- Top-down hammer blow test procedures show room for improvement in more consistent shear wave velocity data throughout the soil column. Also, supplemental lab testing (such as triaxial testing) could be employed to provide better understanding of the soil properties.

- Improvement of table calibration procedures in creating command signals may provide a better match between desired input motions and actual output motions. One suggestion is to calibrate the motions at intensity levels close to that of the desired motion, and subsequently wait a few days before testing to allow the soil to regain any stiffness lost during the calibration procedure.

\subsection{Opportunities for Future Research}

Outlined here are opportunities for future research using the existing structural model and the dataset from this study as well as suggestions for future investigations using this new shake table testing platform:

- Use the dataset generated in this study to calibrate non-linear models. 
- Perform rigorous analyses on the structural model to gain understanding of the structural implications at the observed distortion levels.

- Perform physical tests on the model structure to obtain parameters such as the stiffness, material damping, and soil-structure interface adhesion for use in further numerical modeling.

- Perform shake table tests on multiple tunnel structures with differing flexibility ratios and embedment depths.

- Investigate radiation damping effects and near-field modulus degradation.

- Use pressure transducers on the exterior of the model to explore dynamic earth pressure distributions.

- Investigate SSI effects on other types of structures such as retaining walls, shallow foundations, and deep foundations.

- Explore the effects of variable soil layering.

- Use a modeling of models approach to explore the validity of the proposed scaling relations for different geometric scale factors.

The proposed shake table testing platform constitutes a valuable tool which should be utilized to the fullest extent possible, and will hopefully see many improvements as further experience and understanding regarding soil-structure interaction testing is gained. 


\section{REFERENCES}

Amberg, W., and Russo, M. (2001). "Seismic Design of Underground Structures The Bolu Tunnel." Lombardi SA Engineering Limited.

An, X., Shawky, A. A., and Maekawa, K. (1997). "The collapse mechanism of a subway station during the Great Hanshin earthquake." Cement and concrete composites, 19(3), 241-257.

Arango-Greiffenstein, I. (1971). "Seismic stability of slopes in saturated clay.." Ph.D. Dissertation, Univ. of California, Berkeley.

Biscontin, G., and Pestana, J. M. (1999). Influence of peripheral velocity on undrained shear strength and deformability characteristics of a bentonite-kaolinite mixture. Research Report UCB/GT/99-19, Dep. of Civil and Envir. Engng., University of California, Berkeley.

Boore, D. M., and Bommer, J. J. (2005). "Processing of strong-motion accelerograms: needs, options and consequences." Soil Dynamics and Earthquake Engineering, 25(2), 93-115.

Bowles, J. E. (1995). Foundation Analysis and Design. McGraw-Hill Science/Engineering/Math.

Buehrle, R., Gibbs, G., Klos, J., and Mazur, M. (2003). "Modeling and validation of damped plexiglas windows for noise control." 44 th AIAA/ASME/ASCE/AHS/ASC Structures, Structural Dynamics, and Materials Conference.

Che, A., and Iwatate, T. (2002). "Shaking table test and numerical simulation of seismic response of metro structures." Structures under Shock and Impact VII, 11, 367-376.

Che, A., Iwatate, T., and Ge, X. (2006). "Study on dynamic response of embedded long span corrugated steel culverts using scaled model shaking table tests and numerical analyses." Journal of Zhejiang University-Science A, 7(3), 430-435.

Chung, S. F., Randolph, M. F., and Schneider, J. A. (2006). "Effect of penetration rate on penetrometer resistance in clay." Journal of Geotechnical and Geoenvironmental Engineering, 132, 1188.

Dalgıç, S. (2002). "Tunneling in squeezing rock, the Bolu tunnel, Anatolian Motorway, Turkey.” Engineering Geology, 67(1-2), 73-96. 
Dickenson, S. E. (1994). Dynamic response of soft and deep cohesive soils during the Loma Prieta Earthquake of October 17, 1989. University of California, Berkeley. Gruber, A. (1996). "Stress-Strain Testing of a Model Soil for Earthquake Simulation." Rpt. to the Dept. of Civil Eng., University of California, Berkeley.

Hashash, Y. M. A. (2001). "Seismic behavior of underground structures and site response." Earthquake engineering frontiers in the new millennium: Proceedings of the China-US Millennium Symposium on Earthquake Engineering, Beijing, 8-11 November 2000, 243.

Hashash, Y. M., Hook, J. J., Schmidt, B., and I-Chiang Yao, J. (2001). "Seismic design and analysis of underground structures." Tunnelling and Underground Space Technology incorporating Trenchless Technology Research, 16(4), 247-293.

Hashash, Y. M., Park, D., and Yao, J. I. (2005). "Ovaling deformations of circular tunnels under seismic loading, an update on seismic design and analysis of underground structures." Tunnelling and Underground Space Technology incorporating Trenchless Technology Research, 20(5), 435-441.

Hassoun, N. M. (2001). Structural Concrete: Theory and Design. Prentice Hall.

Hibbitt, K., and et al. (2001). ABAQUS/Standard: user's manual. Hibbitt, Karlsson \& Sorensen.

Huang, T. H., Ho, T., Chang, C., Yao, X., Chang, Q., and Lee, H. (1999). “Quick investigation and assessment on tunnel structures after earthquake, and the relevant reinforced methods (in Chinese)." Report for the Public Construction Commission, Taipei, Taiwan.

Hudson, M., Idriss, I. M., and Beikae, M. (1994). "QUAD4M: a computer program to evaluate the seismic response of soil structures using finite element procedures and incorporating a compliant base." Center for Geotechnical Modeling, Department of Civil and Environmental Engineering, University of California, Davis, CA.

Hulme, T. W., Shirlaw, J. N., and Hwang, R. N. (1990). "Settlements during the underground construction of the Singapore MRT." Proceeding 10th Southeast Asian Geotechnical Conference, Taipei: Special Taiwan Session, 521-526.

Huo, H., Bobet, A., Fernandez, G., and Raml'irez, J. (2006). "Analytical solution for deep rectangular structures subjected to far-field shear stresses." Tunnelling and Underground Space Technology incorporating Trenchless Technology Research, 21(6), 613-625.

Huo, H., Bobet, A., Fernandez, G., and Ramirez, J. (2004). "Seismic Evaluation of the Failure of the Daikai Station During the Kobe Earthquake." 11 th International Conference on Soil Dynamics and Earthquake Engineering and the $3 \mathrm{rd}$ International Conference on Earthquake Geotechnical Engineering Proceedings. 
Huo, H., Bobet, A., Fernández, G., and Ramirez, J. (2005). "Load Transfer Mechanisms between Underground Structure and Surrounding Ground: Evaluation of the Failure of the Daikai Station." Journal of Geotechnical and Geoenvironmental Engineering, 131, 1522.

Hwang, J. H., and Lu, C. C. (2007). "Seismic capacity assessment of old Sanyi railway tunnels." Tunnelling and Underground Space Technology incorporating Trenchless Technology Research, 22(4), 433-449.

Iai, S. (1989). "Similitude for shaking table test on soil-structure-fluid model in $1 \mathrm{~g}$ gravitational field." Soils and Foundations, 29(1), 105-118.

Idriss, I. M. (1990). "Response of soft soil sites during earthquakes.” Proc. H. Bolton Seed Memorial Symposium, 273-289.

Idriss, I. M., and Sun, J. I. (1992). "SHAKE91: A computer program for conducting equivalent linear seismic response analyses of horizontally layered soil deposits." User's guide.

Iida, H., Hiroto, T., Yoshida, N., and Iwafuji, M. (1996). "Damage to Daikai subway station." Soils and foundations, 283-300.

Kontoe, S., Zdravkovic, L., Potts, D. M., and Menkiti, C. O. (2008). "Case study on seismic tunnel response.” Canadian Geotechnical Journal, 45(12), 1743-1764.

Kozak, A., Sedarat, H., and Krimotat, A. (1999). "Alameda Tubes seismic retrofit studies." Computers and Structures, 72(1-3), 233-252.

Kramer, S. L. (1996). Geotechnical Earthquake Engineering. Prentice Hall.

Kuesel, T. R. (1969). "Earthquake design excitation for subways.” J. Struct. Eng. ASCE, 95, 1213-1231.

Li, Y., Emeriault, F., Kastner, R., and Zhang, Z. X. (2009). "Stability analysis of large slurry shield-driven tunnel in soft clay." Tunnelling and Underground Space Technology, 24(4), 472-481.

Lu, C. C., and Hwang, J. H. (2008). "Damage of New Sanyi Railway Tunnel During the 1999 Chi-Chi Earthquake.” Geotechnical Special Publication, 181.

"LVDT Basics." (2003). Macro Sensors, Comercial, , $<$ http://www.macrosensors.com/lvdt_macro_sensors/lvdt_tutorial/lvdt_primer.pdf $>$ (Feb. $16,2010)$.

Lysmer, J., Udaka, T., Tsai, C. F., and Seed, H. B. (1975). "FLUSH: a computer program for approximate 3-D analysis of soil-structure interaction problems." 
Matsui, J., Ohtomo, K., and Kanaya, K. (2004). "Development and Validation of Nonlinear Dynamic Analysis in Seismic Performance Verification of Underground RC Structures." Journal of Advanced Concrete Technology, 2(1), 25-35.

Meymand, P. J. (1998). "Shaking table scale model tests of nonlinear soil-pilesuperstructure interaction in soft clay." Ph.D. Dissertation, Univ. of California, Berkeley.

Moncarz, P. D., and Krawinkler, H. (1981). "Theory and application of experimental model analysis in earthquake engineering." John A. Blume Earthquake Engineering Center technical report, 50.

Monsees, J. E., and Merritt, J. L. (1991). "Earthquake considerations in design of the Los Angeles Metro." Lifeline Earthquake Engineering, 75-88.

Morante, R. J. (2007). Recommendations for Revision of Seismic Damping Values in Regulatory Guide 1.61. U.S. Nuclear Regulatory Commission, Office of Nuclear Regulatory Research.

Muroya, K., Haya, H., Nishiyama, S., Nishimura, A., Sugiyama, I., and Kawama, I. (1998). "The study on seismic behavior of model of box type tunnel constructed by opencut method with slip layer, Part 1: The shaking table tests." The 10th Earthquake Engineering Symposium, 1969-1974.

Nam, S. H., Song, H. W., Byun, K. J., and Maekawa, K. (2006). "Seismic analysis of underground reinforced concrete structures considering elasto-plastic interface element with thickness.” Engineering Structures, 28(8), 1122-1131.

Nishiyama, S., Muroya, K., Haya, H., Tanamura, S., and Nishimura, A. (1999). "Seismic Design of Cut and Cover Tunnel Based on Damage Analyses and Experimental Studies." Quarterly Report of RTRI, 40(3), 158-164.

Ordonez, G. A. (2000). "SHAKE2000: A computer program for the 1-D analysis of geotechnical earthquake engineering problems." SHAKE2000, User's Manual, March 2004, $316 \mathrm{~s}$.

O'Rourke, T. D., Goh, S. H., Menkiti, C. O., and Mair, R. J. (2001). "Highway tunnel performance during the 1999 Duzce earthquake." Proceedings of the International Conference of Soil Mechanics and Geotechnical Engineering, 1365-1368.

Ostadan, F., and Penzien, J. (2001). "Seismic design of cut-and-cover section of the bay area rapid transit extension to San Francisco Airport." Proceedings of 2nd US-Japan SSI Workshop, 1-16.

Parra-Montesinos, G. J., Bobet, A., and Ramirez, J. A. (2006). "Evaluation of soilstructure interaction and structural collapse in Daikai subway station during Kobe earthquake." ACI structural journal, 103(1), 113. 
Penzien, J. (2000). "Seismically induced racking of tunnel linings.” Earthquake Engineering \& Structural Dynamics, 29(5), 683-691.

Penzien, J., and Wu, C. L. (1998). "Stresses in linings of bored tunnels." Earthquake engineering and structural dynamics, 27(3), 283-300.

Randolph, M. F., and Houlsby, G. T. (1984). "Limiting pressure on a circular pile loaded laterally in cohesive soil." Geotechnique, 34(4), 613-623.

Schnabel, P. B., Lysmer, J., and Seed, H. B. (1972). "SHAKE: A computer program for earthquake response analysis of horizontally layered sites.” Report EERC, 72, 12.

Seed, H. B., and Whitman, R. V. (1970). "Design of earth retaining structures for dynamic loads." Lateral Stresses in the Ground and Design of Earth Retaining Structures, 103-147.

Seed, H. B., and Clough, R. W. (1963). "Earthquake resistance of sloping core dams." $J$. Soil Mech. and Found. Div, 89(1), 209-244.

Sitar, N. (1995). "Geotechnical reconnaissance of the effects of the January 17, 1995, Hyogoken-Nanbu Earthquake Japan.” Earthquake Engineering Research Center, Univ. of California at Berkeley, Rep. No. UCB/EERC-95/01.

"Specifications for Earthquake Resistant Design of Submerged Tunnels." (1975). Japanese Society of Civil Engineers (JSCE).

Stewart, D. P., and Randolph, M. F. (1991). "A new site investigation tool for the centrifuge." Centrifuge 91 (eds. Ko and McLean), Balkema, Rotterdam, 531-538.

Sun, J. I., Golesorkhi, R., and Seed, H. B. (1988). "Dynamic moduli and damping ratios for cohesive soils." Report No. EERC, 88, 15.

Technical Manual for Design and Construction of Road Tunnels. (2009). Federal Highway Administration (FHWA), United States.

Vucetic, M., and Dobry, R. (1991). "Effect of soil plasticity on cyclic response." Journal of Geotechnical Engineering, 117(1), 89-107.

Wang, J. J. (1993). “Seismic design of tunnels.” Parsons Brinckerhoff Monograph, 7.

Wang, W. L., Wang, T. T., Su, J. J., Lin, C. H., Seng, C. R., and Huang, T. H. (2001). "Assessment of damage in mountain tunnels due to the Taiwan Chi-Chi Earthquake." Tunnelling and Underground Space Technology incorporating Trenchless Technology Research, 16(3), 133-150. 
Wang, Z. Z., Gao, B., Jiang, Y. J., and Yuan, S. (2009). "Investigation and assessment on mountain tunnels and geotechnical damage after the Wenchuan earthquake." Science in China Series E: Technological Sciences, 52(2), 546-558.

Wartman, J. (1996). "A laboratory Study of the Effects of Fly Ash on the Geotechnical Properties of Soft Clay.” M.E. Thesis, University of California, Berkeley.

Wolf, J. P., and Song, C. (2002). "Some cornerstones of dynamic soil-structure interaction." Engineering Structures, 24(1), 13-28.

Yashiro, K., Kojima, Y., and Shimizu, M. (2007). "Historical earthquake damage to tunnels in Japan and case studies of railway tunnels in the 2004 Niigataken-Chuetsu earthquake." Quarterly Report of RTRI, 48(3), 136-141. 


\section{APPENDIX A}

Detailed Material Mixing and Placement Procedures 


\section{Mixing Procedures}

All of the material used is in 50 pound quantities, bags for kaolinite and bentonite and 5 gallon plastic buckets for the fly ash. This simplified the mixing procedure, because the batches can all be created in terms of bag quantities instead of by weight. Kaolinite was used as the base material for this mixing philosophy, so all batches were mixed and quantified in terms of the number of kaolinite bags in the mix. Buckets filled to a line that denoted a specific quantity of water were counted to keep track of the amount of water added to the tank. A simple spreadsheet solution was used which provides material quantities according the desired amount of kaolinite bags for a particular soil batch. Based on the quantities used and a target water content of $125 \%$, the largest soil batch the mixer can handle is a 12 bag kaolinite mix. That roughly translates to 6 inches deep of packed material in the test container. This size batch is difficult to maintain over multiple batches. The optimum batch size was found to be a 9 bag kaolinite mix, but this requires more batches to fill the container to the desire level.

The following are step by step procedures for operating the mixer, mixing the material, discharging the material, and packing the material. It is important note that the behavior of the mixer and soil material varies from batch to batch, and adjustments to procedures need to be made accordingly. Proper safety gear such as latex gloves, safety goggles, and respirators should be worn at all times when working with the material, especially due to the highly caustic nature of the class C fly ash.

1. Before operation, check that the discharge valves on the mixer are set to cycle the material through the pipes (the valve handles should be vertical). 


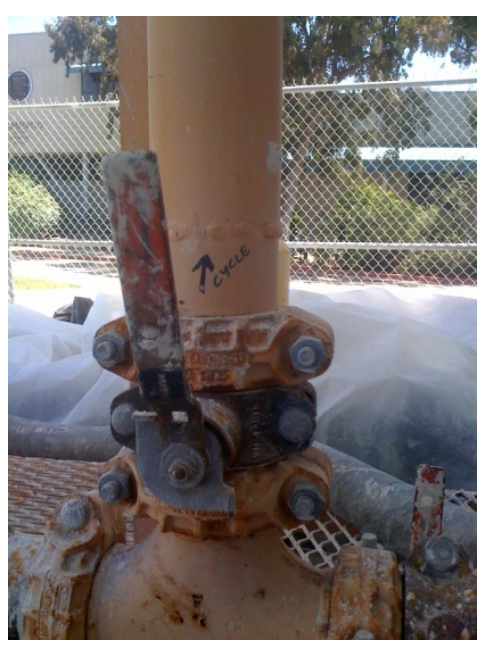

Figure A.1 - Valve set to CYCLE (one of two valves)

2. Fill tank with about half of the water required for the full mix using buckets marked with a known water weight

3. Insert $460 \mathrm{~V}$ plug into the Outlet at the northeast corner of the shake table lab and lock it in the ON position (Figure A.2).

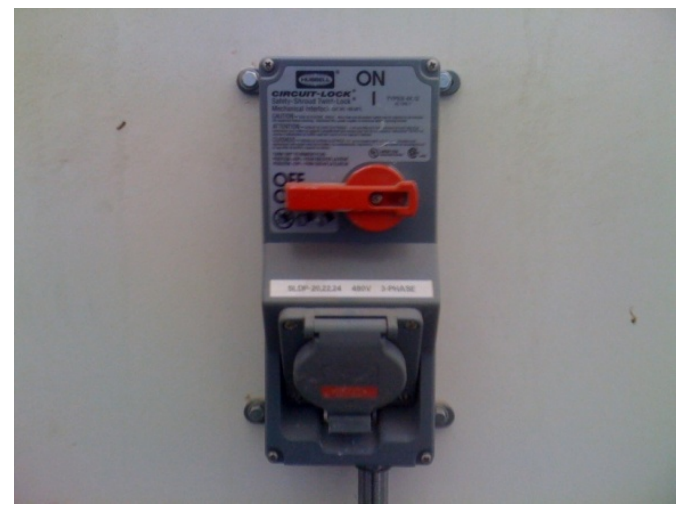

Figure A.2 - 460V power outlet

4. Turn the hydraulic control to the NEUTRAL (Figure A.3a) and press the green ON (Figure A.3b) button to start the mixer. NEUTRAL is between MIX and PUMP. 


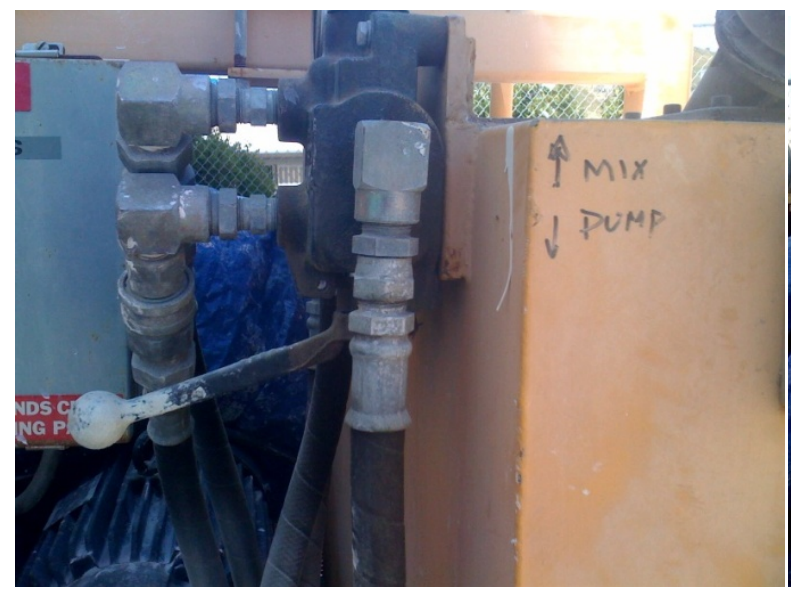

(a)

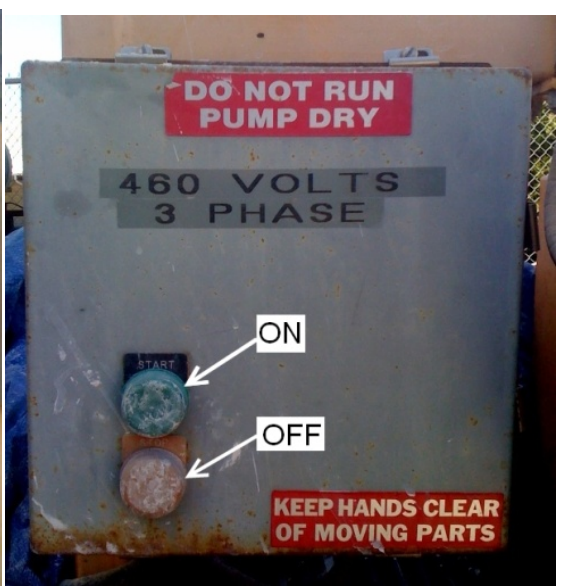

(b)

Figure A.3 - (a) Hydraulic controls and (b) Electrical controls

5. Turn the control to PUMP to cycle the water, be sure that water is cycling through the pump and back into the tank. This is the "Forward" direction of the progressive cavity pump.

6. Mix proportional amounts of dry kaolinite and bentonite together in a large container (e.g. a wheel barrow) in order to achieve a uniform powder mix Figure A.4. Best results seem to come from mixing 1 bag of kaolinite with roughly $1 / 3$ bag of bentonite at a time. Mixing dry material keeps large bentonite clumps from forming in the mixer.

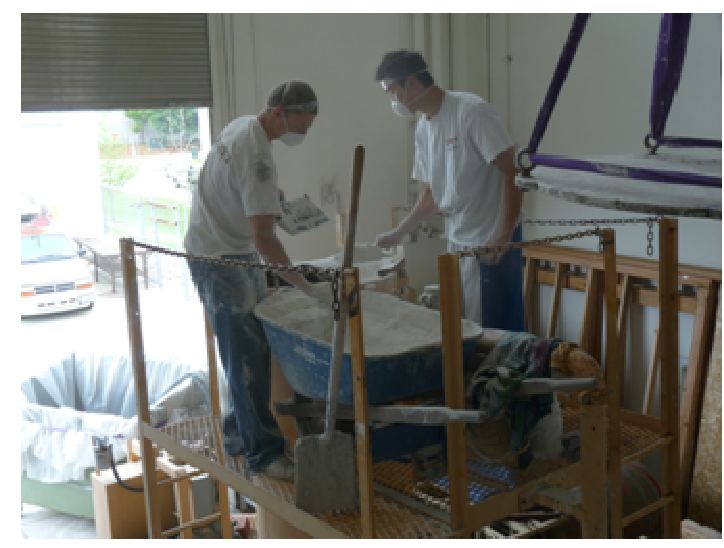

Figure A.4 - Mixing dry material in a wheel barrow and adding it to the tank 
7. Add the powder directly into the tank as the water cycles.

8. Repeat steps 6 and 7 as many times as desired until the tank is full, adding small amounts of water to ease mixing. A couple buckets of water should be left over to aid in adding fly ash near the end of the mixing process.

9. Allow kaolinite/bentonite mix to cycle until a reasonable consistency is achieved. This usually takes at least 2 hours, but possibly more. Monitor the mix until it becomes homogenous throughout.

10. Add the fly ash using a small scooper to the material as it continues to cycle, being careful to distribute it evenly. Small amounts of water set aside can be used to ease spreading of fly ash. This process should be completed quickly, as the fly ash has a dramatic stiffening effect on the soil.

11. Discharge soil before it stiffens excessively. This should be done less than 30 minutes after adding the fly ash.

Other mixing notes:

- Use a platform hung from the overhead crane to transport the material to the top of the mixer (Figure A.5)

- One can manually help mix from up top to get the powder to cycle using a shovel or appropriate tool. Meymand (1998) reported that an electric paddle mixer can be used to greatly facilitate the mixing process; however, such equipment was not available for this research.

- The pump can be set to MIX for small periods of time $(<1 \mathrm{~min})$ in order to help agitate the material. This runs the pump impeller in reverse and cannot be 
sustained for long, because it can quickly cause the pump to run dry, possibly damaging it.

- Adding material using the "dry material hopper" in Figure 4.8 is not recommended because the piping system may back up. The valve for the hopper should remain closed at all times during mixing.

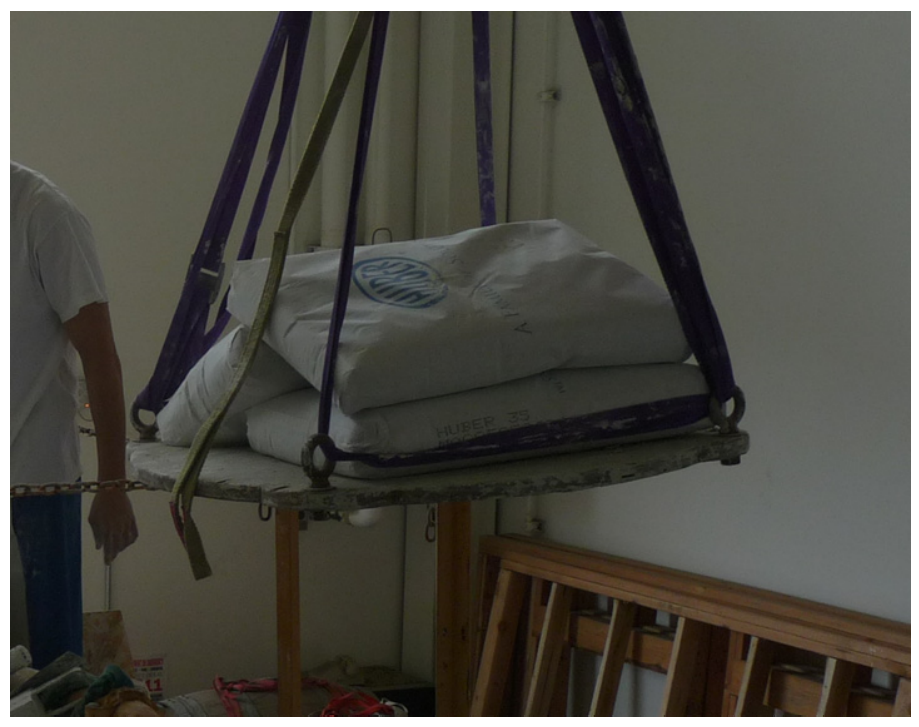

Figure A.5 - Platform hung from crane for transporting material

\section{Discharge Procedures}

Prior to discharge, the 3 inch discharge hose should be setup in such a way that it minimizes kinks and aims into the test container. The ideal solution is to have the mixer far enough away that the hose can be as straight as possible, but this may not be possible due to space limitations. The overhead crane was used in conjunction with a strap to hold and control the hose during discharge Figure A.6. Discharging the soil can be a hazardous and should only be performed if two or more people are present. 


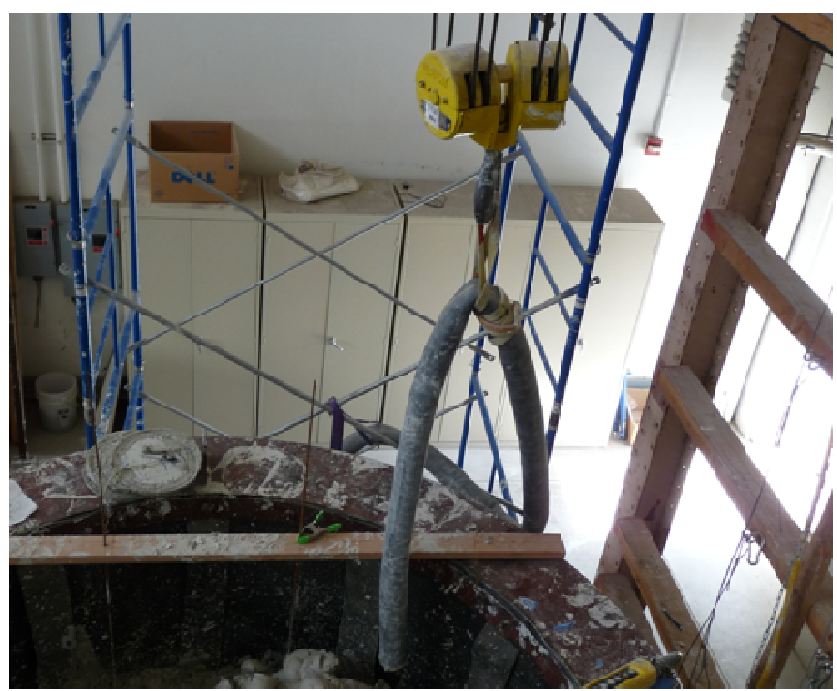

Figure A.6 - Discharge hose held and controlled using the overhead crane

1. Run the pump in reverse for a few seconds to suck material out of the cycling piping (set pump to MIX).

2. Turn the mixer OFF or turn the mixer control to the NEUTRAL position.

3. Open the discharge valves (both valve handles should be horizontal) (Figure A.7).

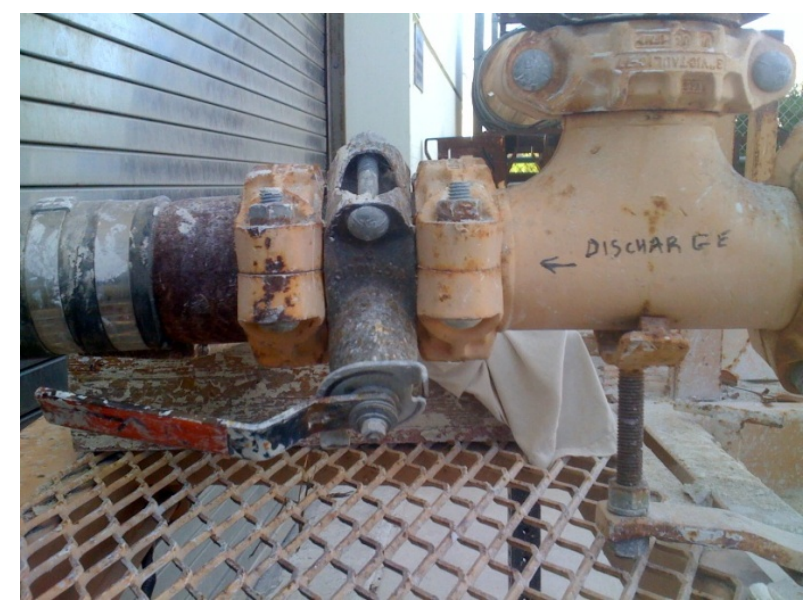

Figure A.7 - Valve set to DISCHARGE (one of two valves)

4. If the hose is not totally secure in the desired position, one person should be in the test container to control the hose during discharge. 
5. When ready, another person turns the mixer ON and the mixer control to PUMP.

6. The material will begin to discharge within a few seconds, and continue discharging until the tank is empty or there is not enough pressure to discharge more material. This problem can be remedied by manually pushing material down with an appropriate tool, or by pressurizing the tank using a pressure cap. The second option was not employed in this research, but the author believes it would be a significant improvement to discharge procedures.

7. Upon completion of discharge, turn the mixer control to MIX to clear the hose of excess pressure and close discharge valves.

8. Begin new soil batch if desired or Turn mixer OFF in the NUETRAL position and remove from power source.

\section{Soil Packing Procedures}

The material packing process requires that the piles of material be dug up and replaced in a more tightly packed fashion. Clay strength is such that standing directly on the clay will cause one to displace material and sink a few centimeters. This promotes the use of small platforms which can be used to stand, sit, or kneel on during packing of the clay. The author used the caps from the fly ash buckets as platforms. It is important that as little bare skin as possible is exposed, due to the extremely high alkalinity of the saturated clay. Small survey flags can be used to mark instrumentation positions. Upon completion of packing, samples should be taken for water content testing or desired lab testing before placement of subsequent soil lifts. 
APPENDIX B

Acceleration Data for Phase I Free-Field Testing 

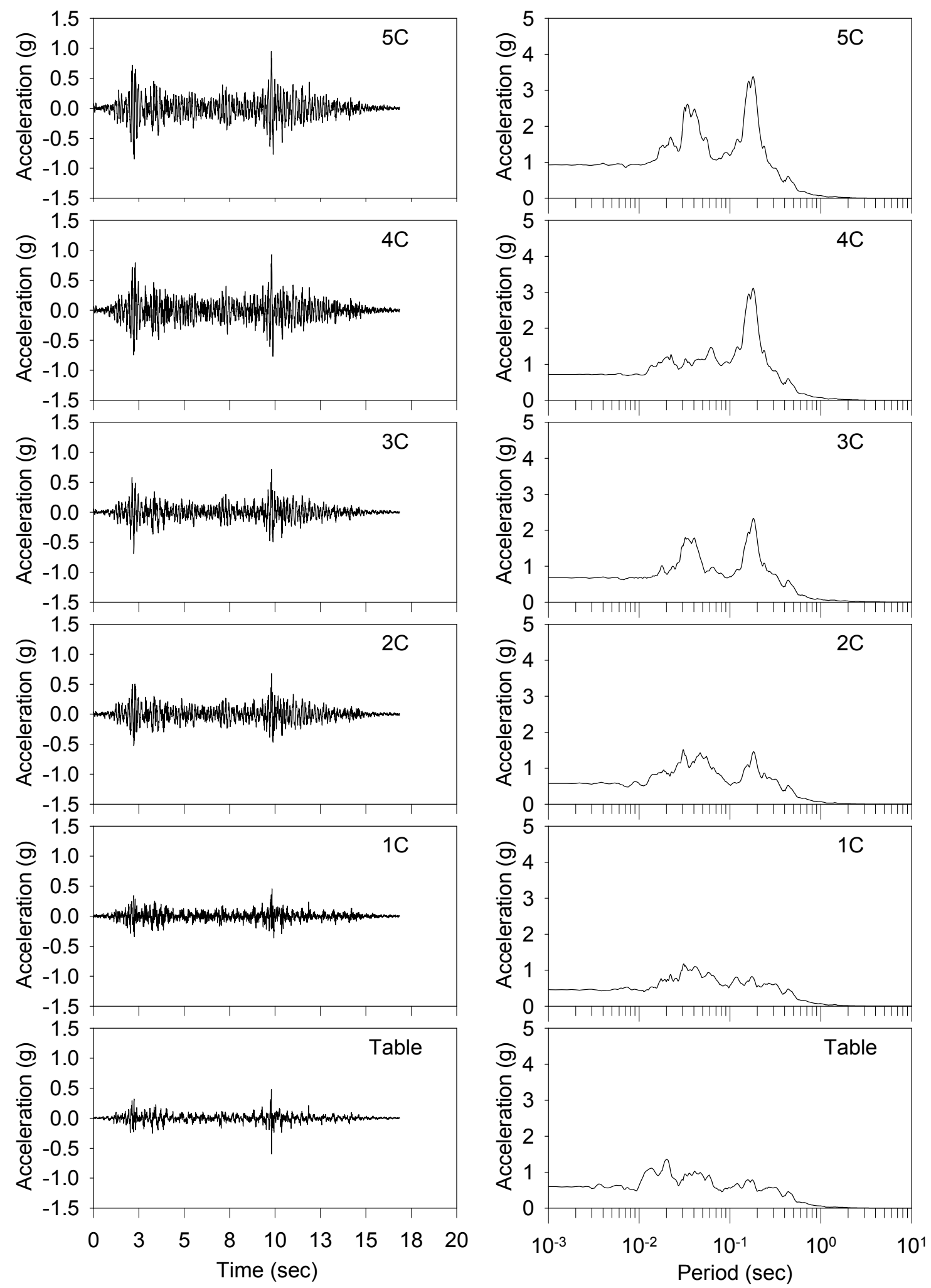

Figure B.1 - Joshua Tree 000 acceleration time histories and 5\% damped response Spectra (test 2_J000) recorded in the center accelerometer array 

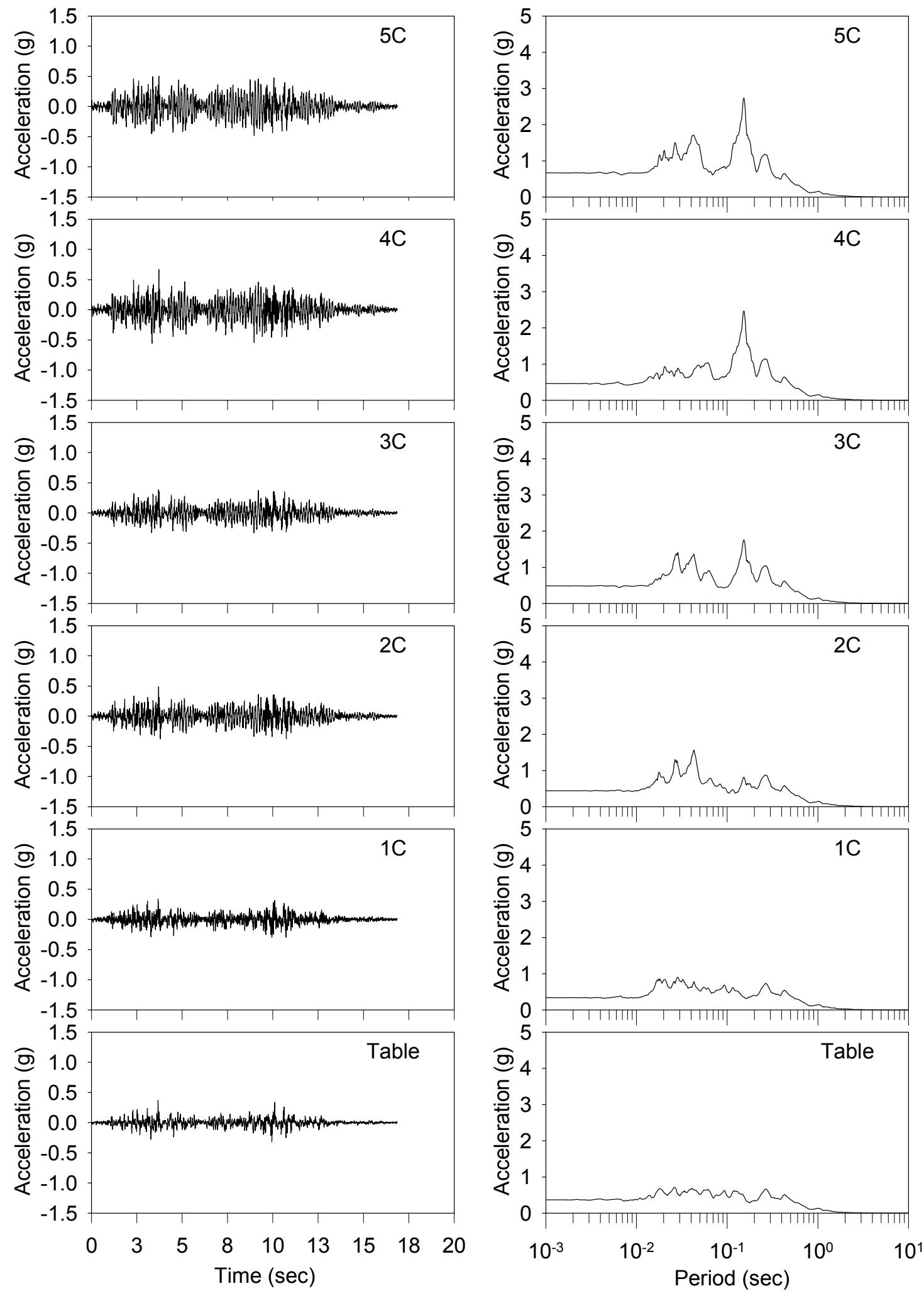

Figure B.2 - Joshua Tree 090 acceleration time histories and 5\% damped response Spectra (test 2_J090) recorded in the center accelerometer array 

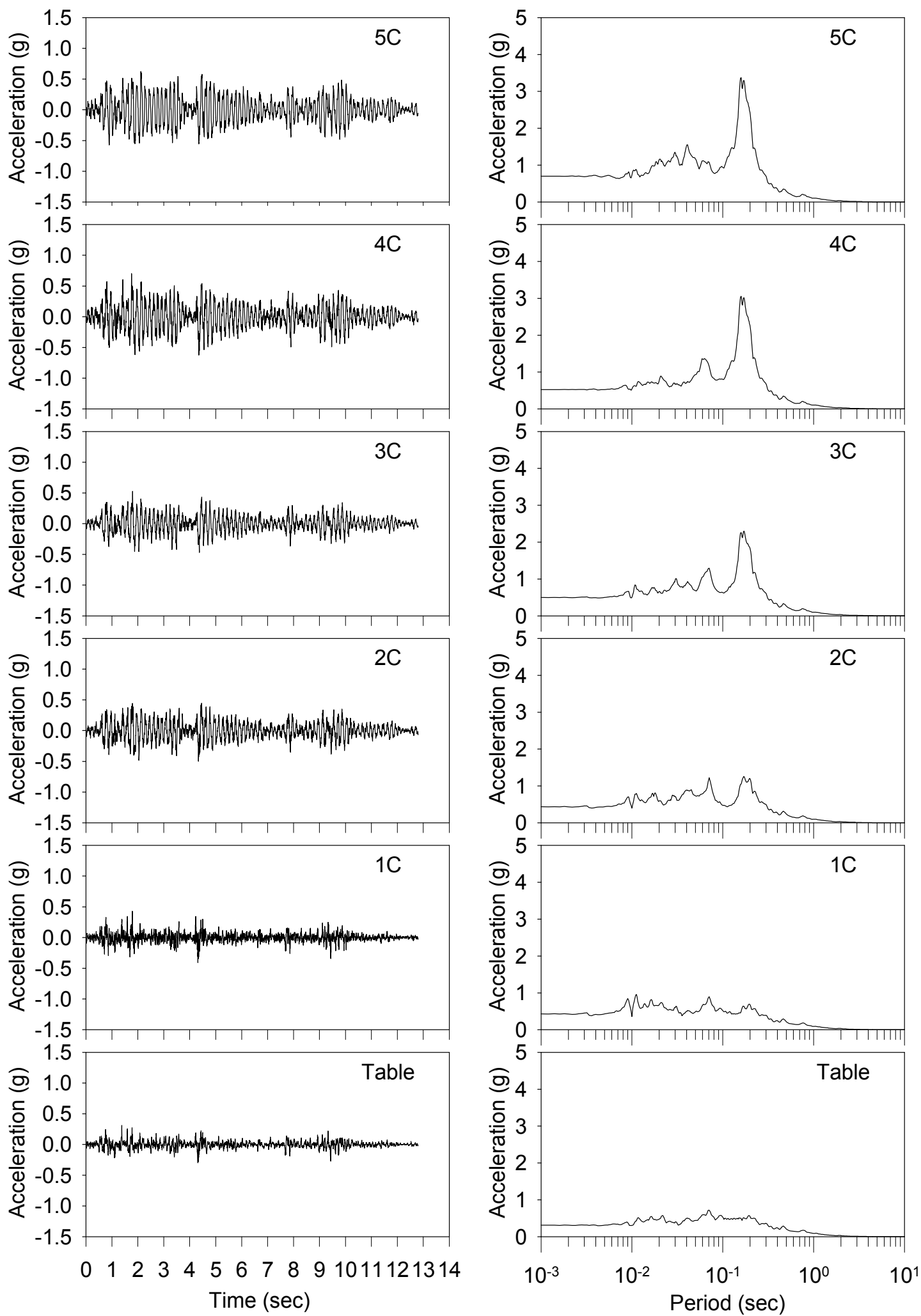

Figure B.3 - El Centro 270 acceleration time histories and 5\% damped response Spectra (test 2_J090) recorded in the center accelerometer array 

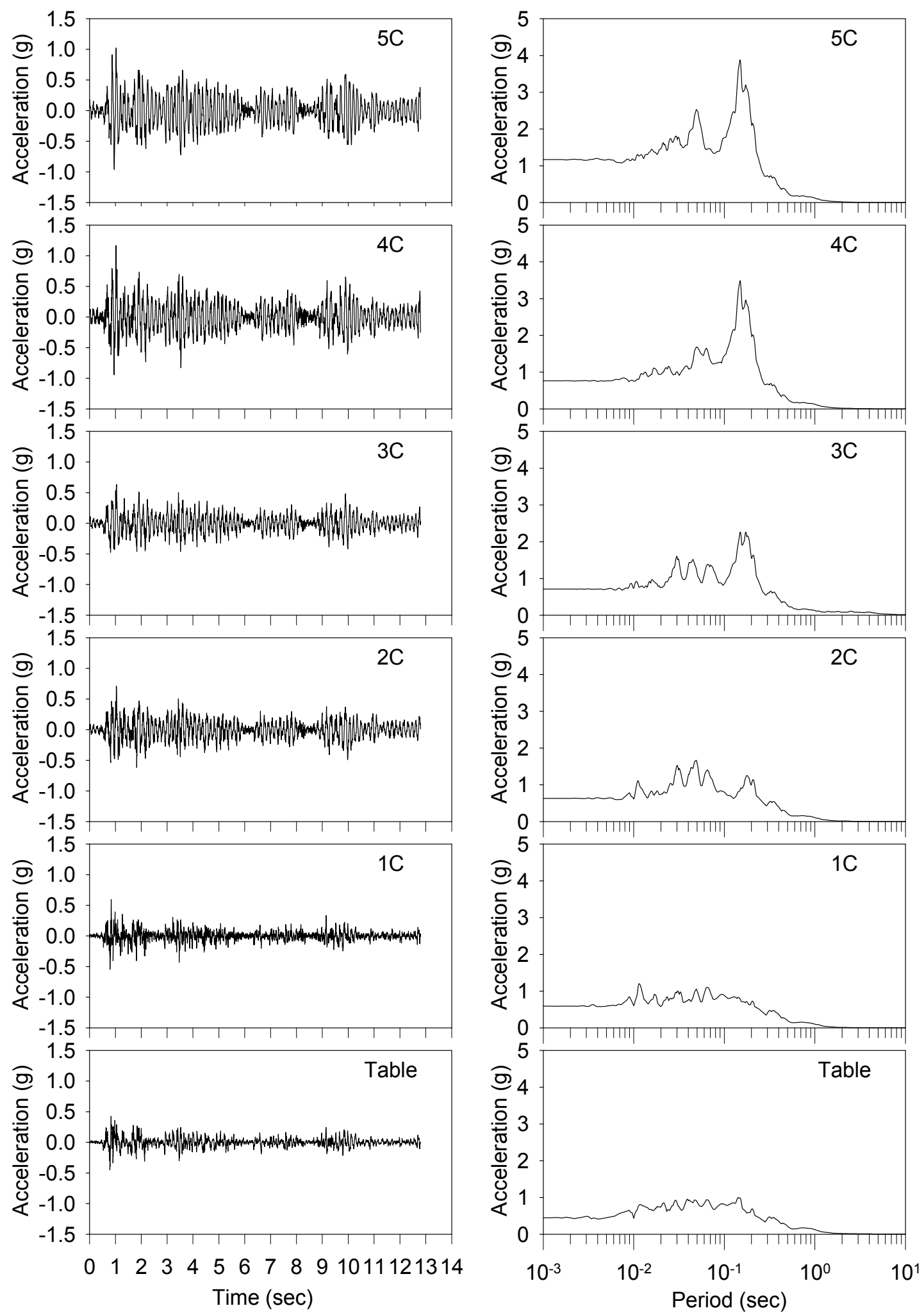

Figure B.4 - El Centro 180 acceleration time histories and 5\% damped response Spectra (test 2_J090) recorded in the center accelerometer array 

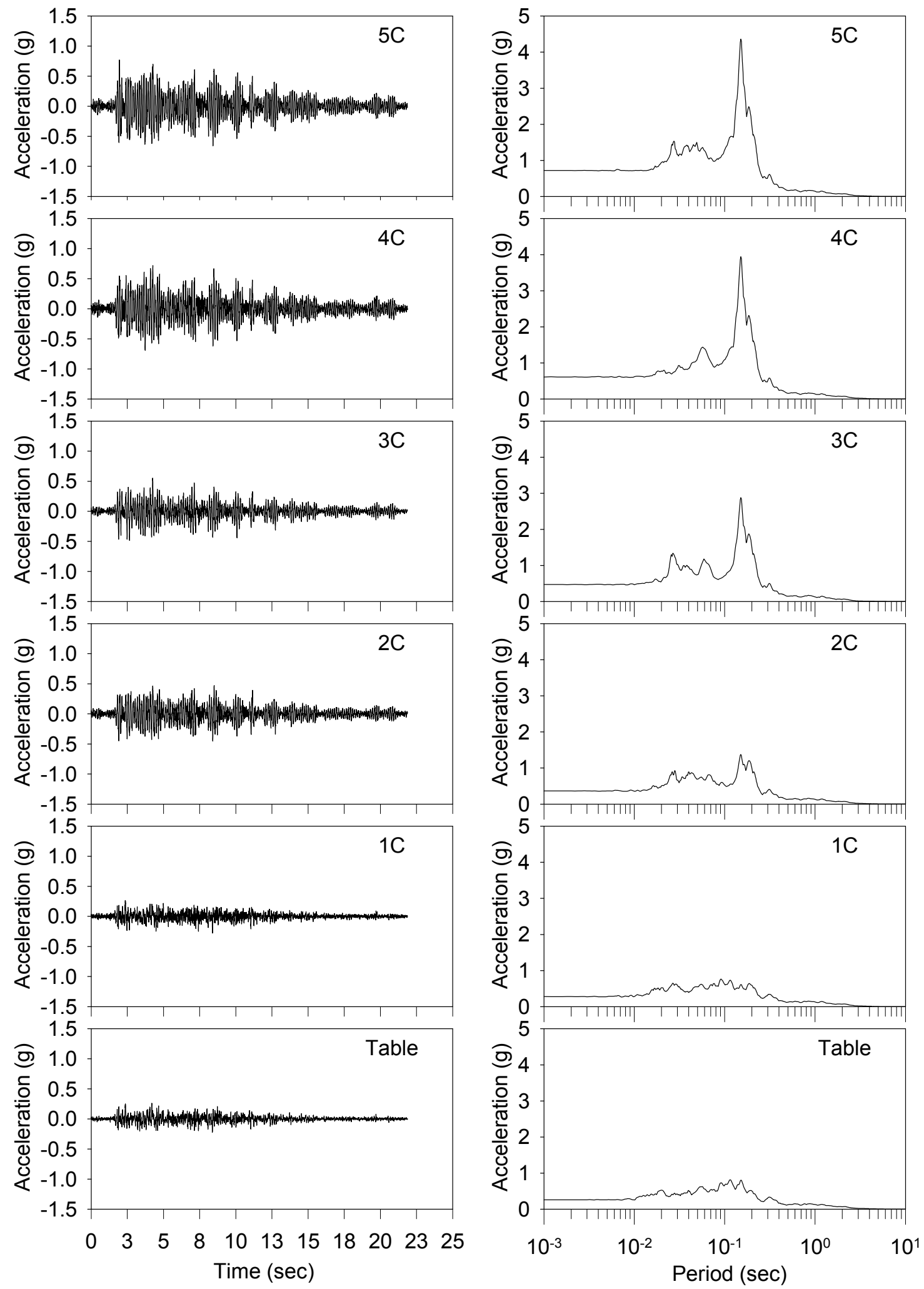

Figure B.5 - TCU075 North acceleration time histories and 5\% damped response Spectra (test 2_J090) recorded in the center accelerometer array 

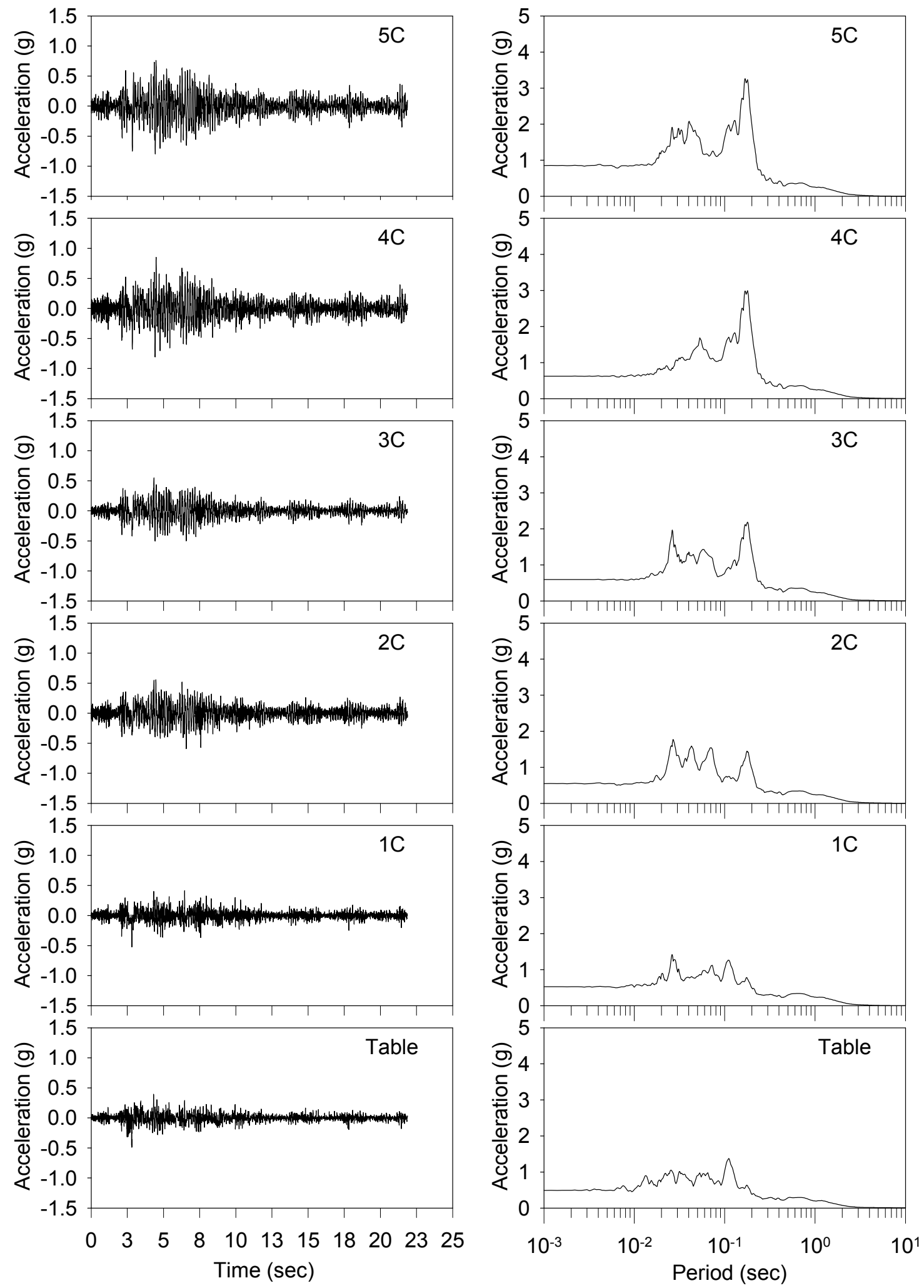

Figure B.6 - TCU075 West acceleration time histories and 5\% damped response Spectra (test 2_J090) recorded in the center accelerometer array 


\section{APPENDIX C}

SHAKE Modeling of Phase I Free-Field Motions 


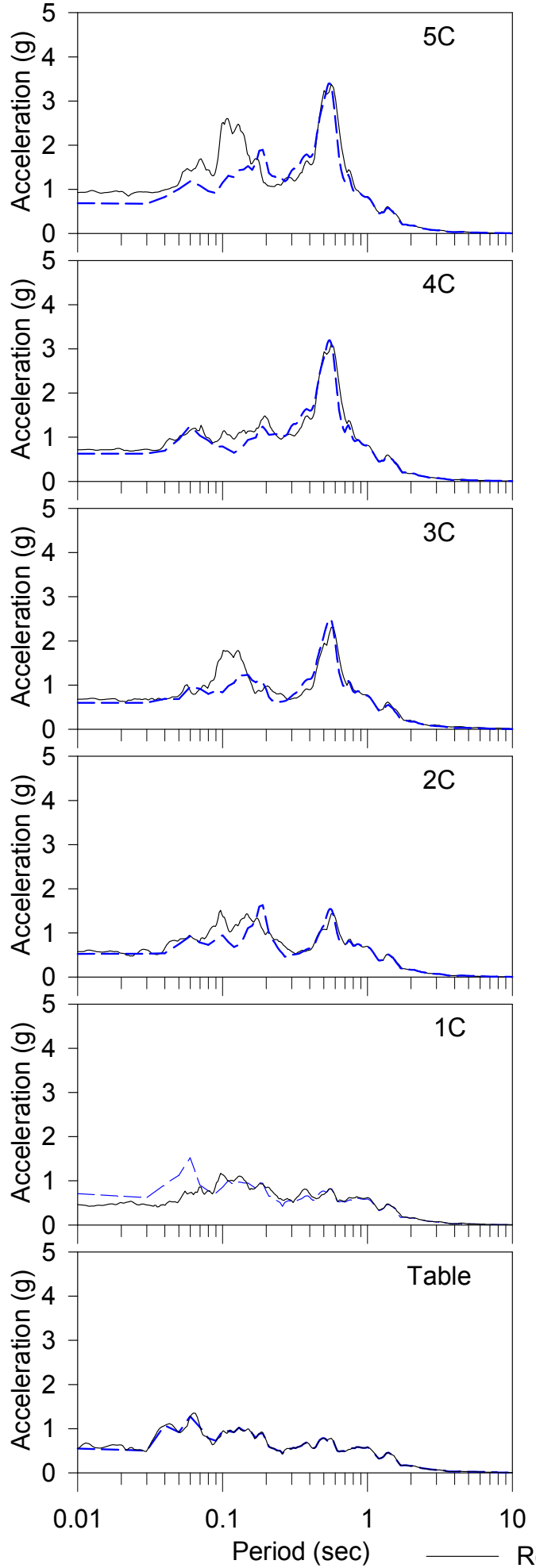

(a)

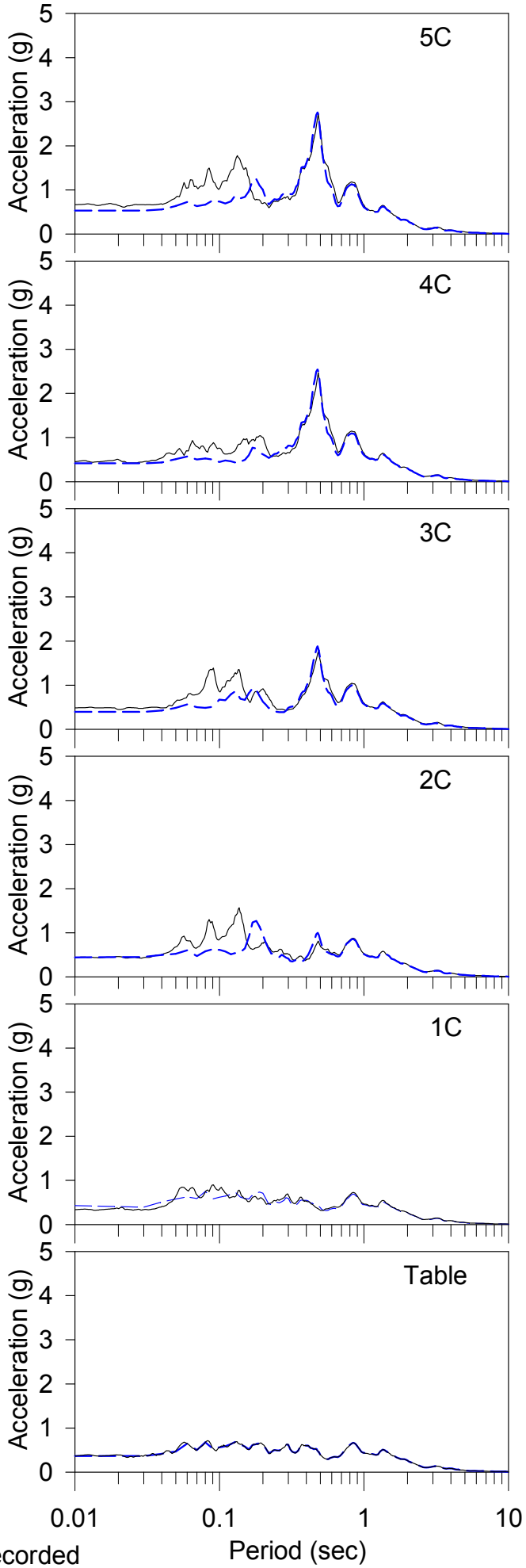

(b)

Figure C.1 - 5\% damped response spectra for a) Joshua Tree 000 (1_J000) and b) Joshua Tree 090 (1_J090) center array recordings versus SHAKE predicted response spectra 


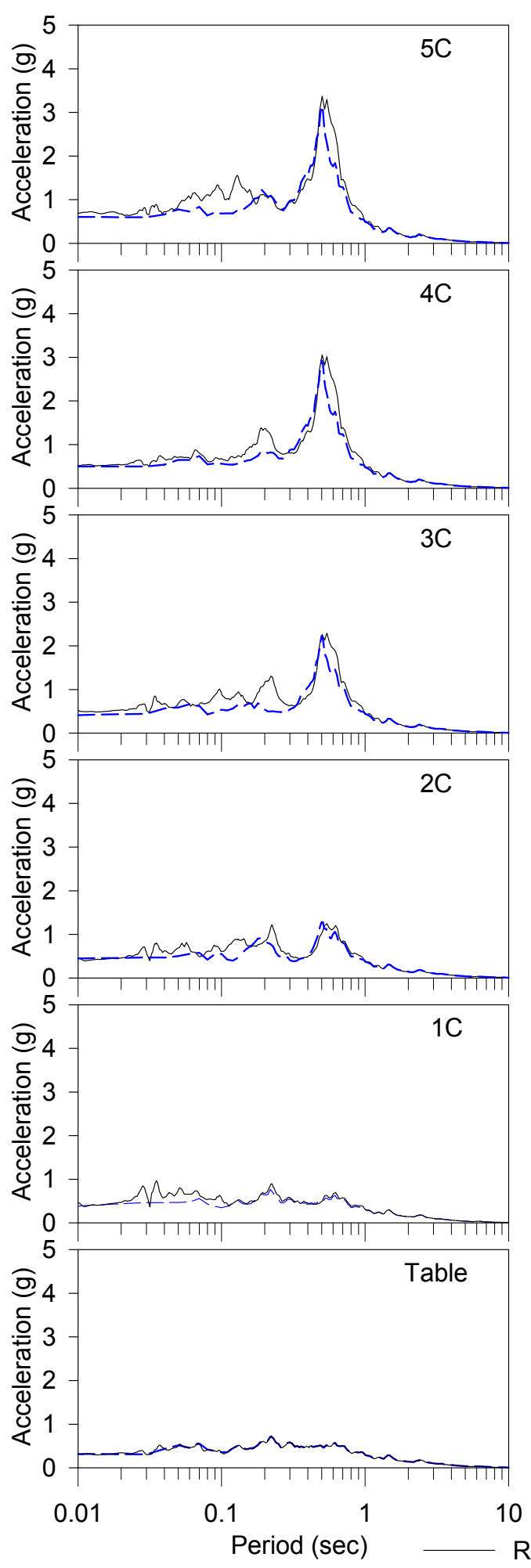

(a)

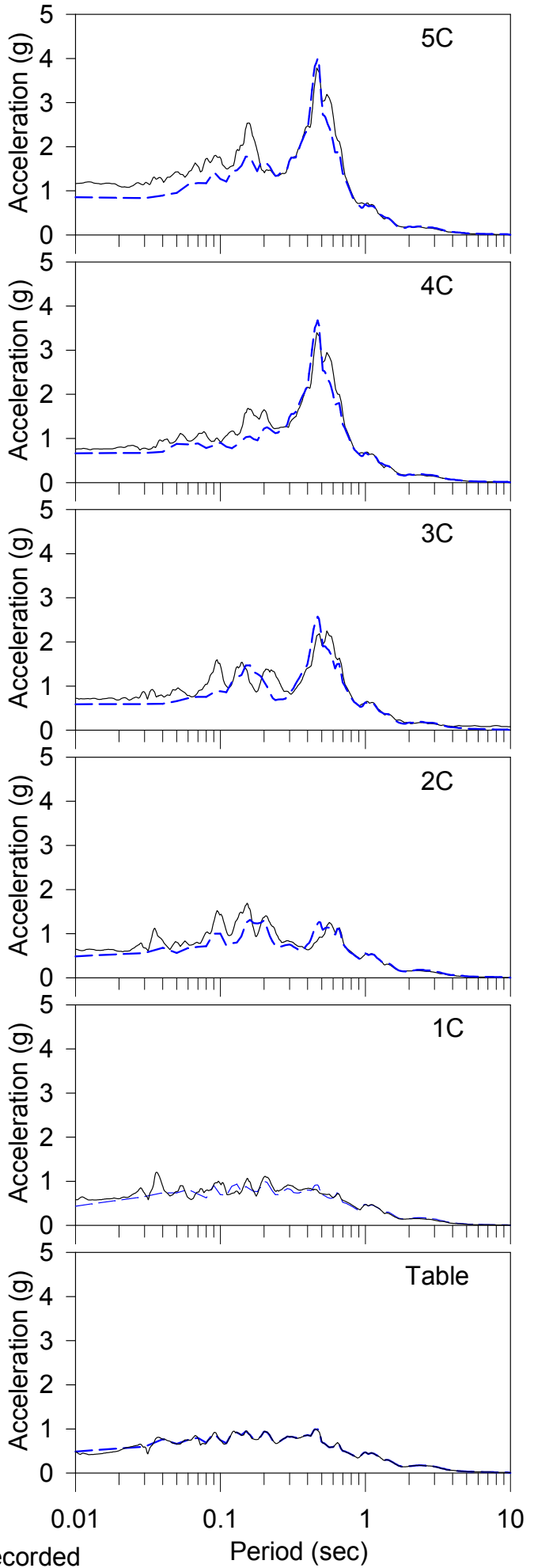

(b)

Figure C.2 - 5\% damped response spectra for a) El Centro 270 (1_E270) and b) El Centro 180 (1_E180) center array recordings versus SHAKE predicted response spectra 

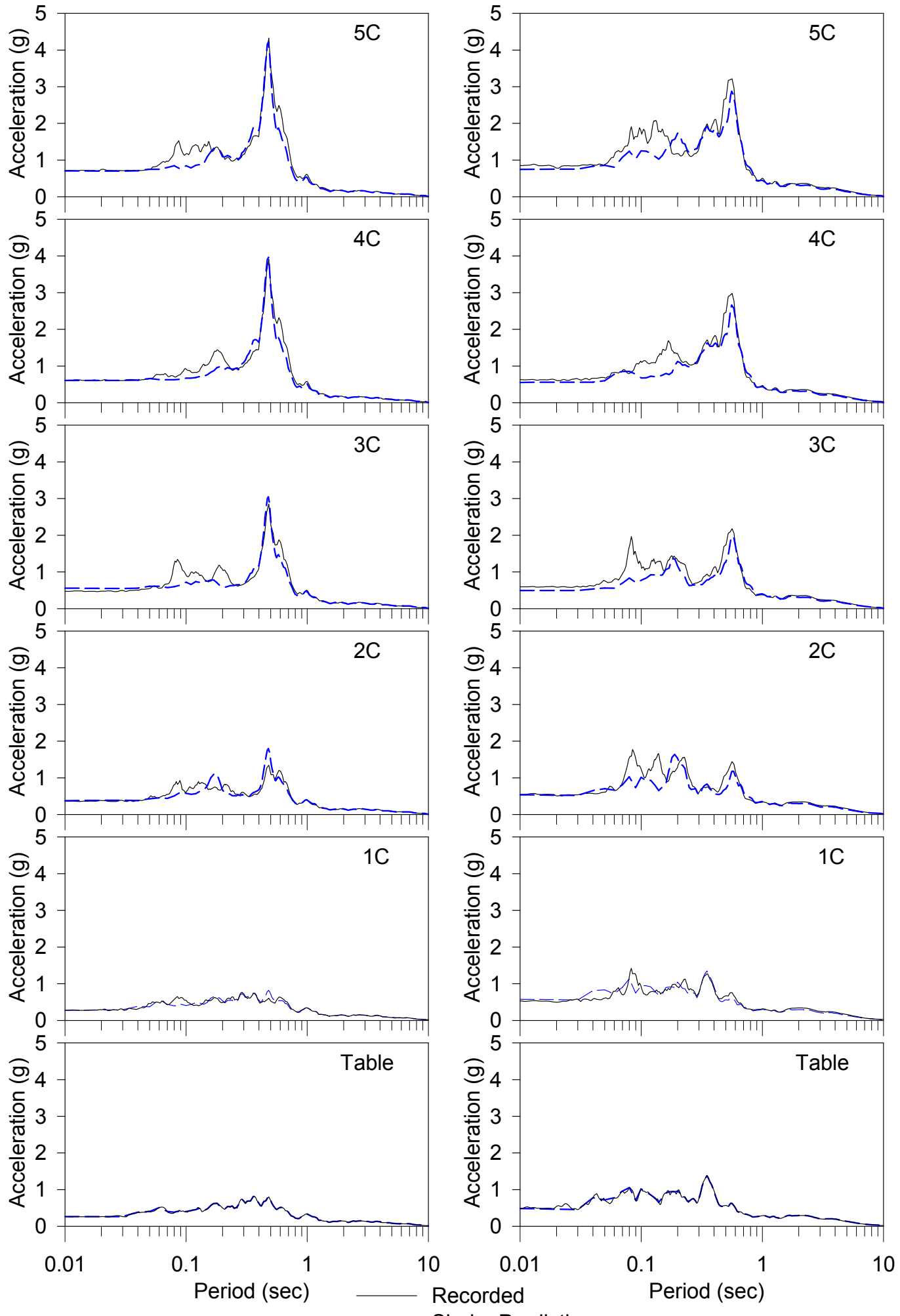

(a)

Shake Prediction

(b)

Figure C.3 - 5\% damped response spectra for a) TCU075 North (1_CN) and b) TCU075 West (1_CW) center array recordings versus SHAKE predicted response spectra 


\section{APPENDIX D}

SHAKE91 Input Time History Error Report: October 6, 2009 
The equivalent linear site response analysis program SHAKE91 (Idriss and Sun 1992) is currently being used in a study at Cal Poly, San Luis Obispo. After extensive use of the program with unsatisfactory results, an investigation into possible software error regarding the input file was performed. It was determined that the format of the input acceleration is important for producing the correct response. Ordóñez (2000) cites a previous error report regarding SHAKE91 posted by Dr. Farhang Ostadan to the NISEE website. The SHAKE2000 manual states: "For correct reading of the time history points, an even number of points should be given per line (i.e. 2, 4, 8, etc.)." We were unable to locate Dr. Ostadan's original error report upon further research.

For our study, the input acceleration time history was initially formatted as a single vertical array (1e15.11), consistently resulting in erroneous site response. This agrees with the statement above that the input file must have an even number of columns (i.e. points per line) to be correct. A check was performed in which all variables in the SHAKE input file were held constant except for the number of columns in the input acceleration time history. The number of columns was varied from 1 to 8 resulting in the site response shown in the following figure. 


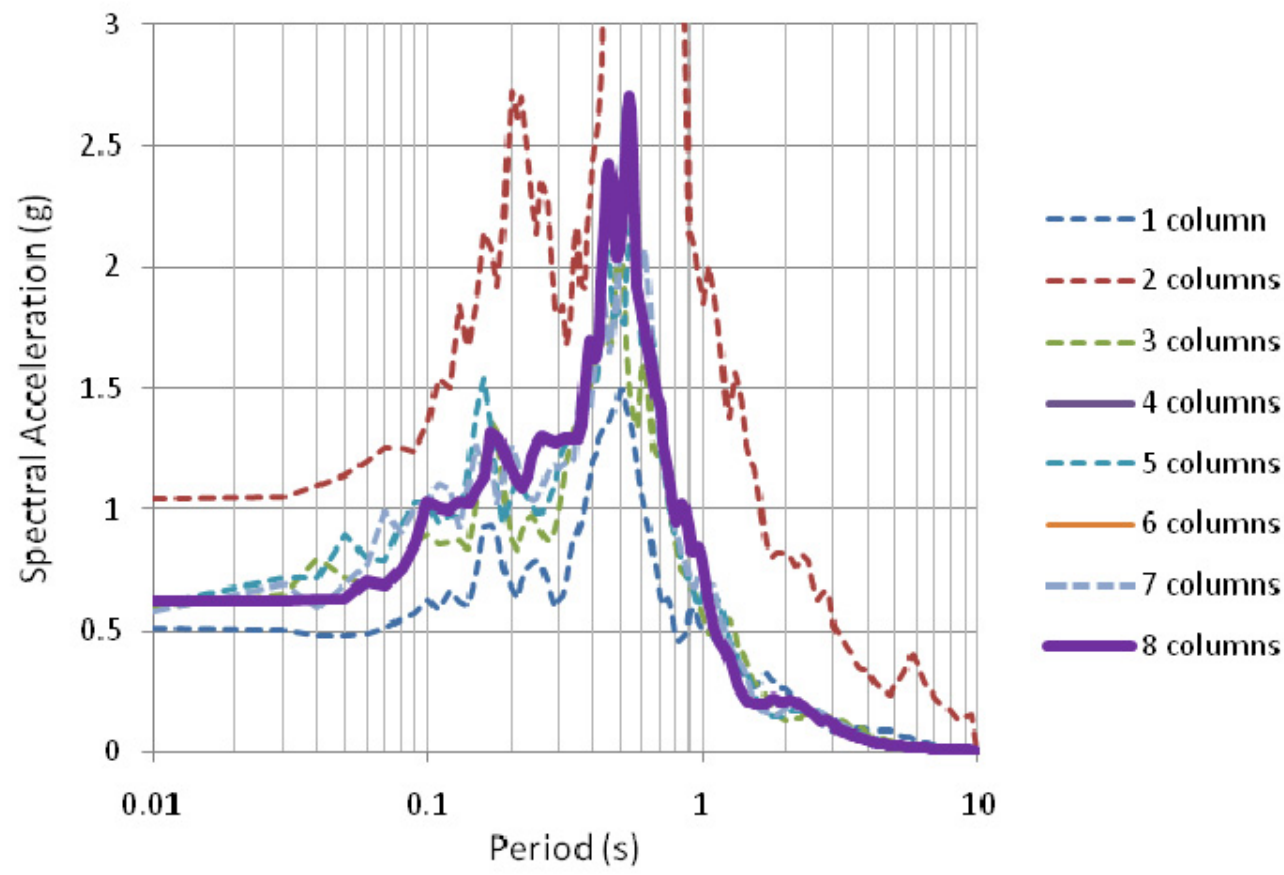

Figure D.1 - Variation of 5\% SHAKE91 5\% damped response spectra with respect to number of columns in input time history

The correct response is generated when the time history input file has 4,6 , or 8 columns, shown above as a thick solid line. The 3,5 , and 7 column input time histories mostly under-predict the site response, while the 2-column time history grossly over-predicts the response. This partly conflicts with the SHAKE2000 manual regarding the 2-column input file.

It is recommended that 8-column acceleration input files be used in all SHAKE91 analyses, as it will result in the correct output response. Ordonez (2000) recommends the format $8 \mathrm{~F} 12.8$ ( 8 columns in the format of 12 spaces for the number with 8 after the decimal) to be used for the input time history. 